\title{
Anchoring Symbols to Percepts in the Fluent Calculus
}

Zur Erlangung des akademischen Grades Doktor rerum naturalium (Dr. rer. nat.)

Vorgelegt an der Technischen Universität Dresden, Fakultät Informatik

Eingereicht am: 26. August 2009, von: Dipl. Inf. MSc Matthias Fichtner, geboren am 21. Januar 1976 in D-04703 Leisnig.

Verteidigt am: 10. Dezember 2009

Betreuender Hochschullehrer: Prof. Dr. rer. nat. habil. Michael Thielscher Zweite Gutachterin: Prof. Silvia Coradeschi (Universität Örebro, Schweden)

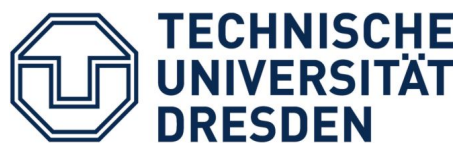




\section{Contents}

1 Introduction and Motivation $\quad 1$

2 Foundations 5

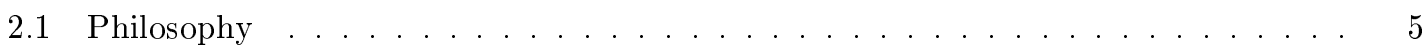

2.1 .1 Philosophy of Language . . . . . . . . . . . . . . . . 5

2.1 .2 Philosophy of Cognition . . . . . . . . . . . . . . . . 6

2.1 .3 Conclusion . . . . . . . . . . . . . . . . . 8

2.2 The Symbol Grounding Problem $\ldots \ldots \ldots \ldots \ldots \ldots \ldots$

2.2 .1 Motivation . . . . . . . . . . . . . . . . . 8

2.2 .2 Description . . . . . . . . . . . . . . . . . . 8

2.2 .3 Characteristics . . . . . . . . . . . . . . . . . . . . 10

2.2 .4 Evaluation . . . . . . . . . . . . . . . . . . . . . . . 10

2.2 .5 Conclusion . . . . . . . . . . . . . . . . . . . . 12

2.3 The Symbol Anchoring Problem . . . . . . . . . . . . . . . . . . 12

2.3.1 Motivation and Description . . . . . . . . . . . . . . . 12

2.3 .2 Terminology . . . . . . . . . . . . . . . . . . . . . . . 14

2.3 .3 Characteristics . . . . . . . . . . . . . . . . . . . . . . 14

2.3 .4 Evaluation . . . . . . . . . . . . . . . . . . . 16

2.3.5 Previous Works . . . . . . . . . . . . . . . . . 17

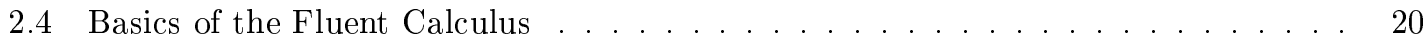

3 Our Approach to Symbol Anchoring 25

3.1 Independence of Information . . . . . . . . . . . . . . . . . 26

3.2 Philosophy of Cognition . . . . . . . . . . . . . . . . . . 27 27

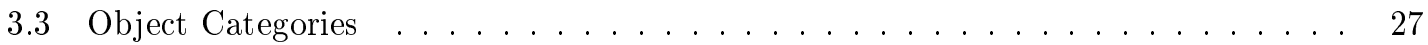

3.4 Each Percept-One Symbol . . . . . . . . . . . . . . . . . . . . . 28

3.5 Uncertainty Representation . . . . . . . . . . . . . . . . . . . . . . . . 29

3.6 Grounding of Predicates . . . . . . . . . . . . . . . . . . . . . 31

3.7 Symbolic Descriptions of Objects . . . . . . . . . . . . . . . . . . 33

3.8 Reference to Objects . . . . . . . . . . . . . . . . . . . . . . . . . . . 34

3.9 Symbol Anchoring Hypothesis . . . . . . . . . . . . . . . . . . . . 35

3.10 Multiple Hypotheses of Correspondences . . . . . . . . . . . . . . . . . . 37

3.11 Object Recognition System _ . . . . . . . . . . . . . . . . . 40

4 Uni-Modal Symbol Anchoring 43

4.1 Representation and Meaning . . . . . . . . . . . . . . . . . 43

4.1 .1 Percept . . . . . . . . . . . . . . . . . . . . 43

4.1.2 Correspondences and Anchors . . . . . . . . . . . . . . . . . . 45

4.1.3 Representing Multiple Hypotheses . . . . . . . . . . . . . . . . . . . 48

4.2 Computing the Space of Hypotheses . . . . . . . . . . . . . . . . . . 49

4.2 .1 Computing the Correspondences . . . . . . . . . . . . . . . 51

4.2 .2 Tracking an Object . . . . . . . . . . . . . . . . . . 52 
4.2.3 Finding and Reacquiring a Correspondence . . . . . . . . . . . . . 55

4.2 .4 Introducing a New Object . . . . . . . . . . . . . . . . . . . . . . . . . .

4.2.5 Complexity of Hypothesis Space . . . . . . . . . . . . . . . 6 61

4.2 .6 Eliminating Inconsistent Hypotheses . . . . . . . . . . . . . . . . . . . . 62

4.2.7 Consistency of Perceptual Estimates . . . . . . . . . . . . . . . . 64

4.2.8 Groundedness of Correspondences . . . . . . . . . . . . . . . 65

4.3 Preferences among Alternative Hypotheses . . . . . . . . . . . . . . . . . . . . 6 67

4.3.1 The Unique Preference Relation . . . . . . . . . . . . . . . . . 68

4.3.2 The Set-Based Preference Relation . . . . . . . . . . . . . . . 69

4.3.3 Heuristics for Preferences . . . . . . . . . . . . . . . . . . . 71

4.4 Summary and Conclusion . . . . . . . . . . . . . . . . . . . . . 77

5 Multi-Modal Symbol Anchoring $\quad \mathbf{7 9}$

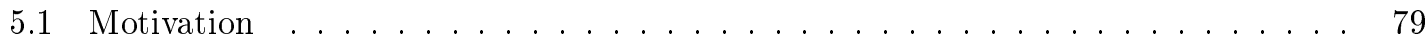

5.2 Object Recognition and Tracking . . . . . . . . . . . . . . . . . . . . 81

5.3 Representation and Meaning . . . . . . . . . . . . . . . . . 82

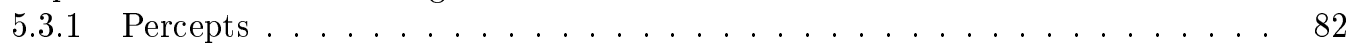

5.3.2 Correspondences and Anchors . . . . . . . . . . . . . . . 83

5.4 Computing the Space of Hypotheses . . . . . . . . . . . . . . . . . . . 85

5.4 Partitioning the Set of Percepts . . . . . . . . . . . . . . . 85

5.4 .2 Proof of Correctness of Predicate PBlock . . . . . . . . . . . . . 87

5.4 .3 Computing the Correspondences . . . . . . . . . . . . . . . . 88

5.4 .4 Tracking an Object . . . . . . . . . . . . . . . . . . . . . . . . . . . . . .

5.4.5 Finding and Reacquiring a Correspondence . . . . . . . . . . . . . . 94

5.4 .6 Introducing a New Object . . . . . . . . . . . . . . . . . . . . . . . . . . . . . . .

5.4 .7 Complexity of Hypothesis Space . . . . . . . . . . . . . . . . . 98

5.4 .8 Eliminating Inconsistent Hypotheses . . . . . . . . . . . . . . . . . . . . . . . . . . . . . . . 99

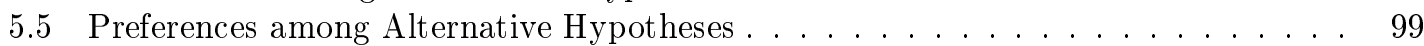

5.6 Characteristics of Our Multi-Modal Approach . . . . . . . . . . . . . . . . . 99

5.6.1 Consistency of Hypotheses . . . . . . . . . . . . . . . 100

5.6 .2 Groundedness of Correspondences . . . . . . . . . . . . . . . . . 102

5.6 .3 Each Percept Is Assigned Some Symbol . . . . . . . . . . . . . . . . 103

5.6.4 Relation between Uni- and Multi-Modal Approaches . . . . . . . . . . . . 104

5.7 Summary and Conclusion . . . . . . . . . . . . . . . . 116

6 Conclusion $\quad 119$

6.1 Evaluation . . . . . . . . . . . . . . . . . . . . . 119

6.1.1 Evaluation with respect to the Symbol Anchoring Problem . . . . . . . 120

6.1.2 Evaluation with respect to the Symbol Grounding Problem . . . . . . . . 121

6.1.3 Summary of Contribution . . . . . . . . . . . . . . . 123

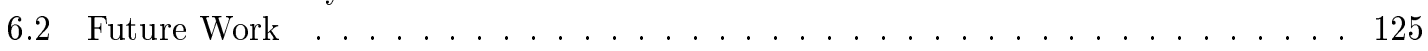

$\begin{array}{lr}\text { A List of Symbols Used } & 129\end{array}$

A.1 Sorts . . . . . . . . . . . . . . . . . . . . 129

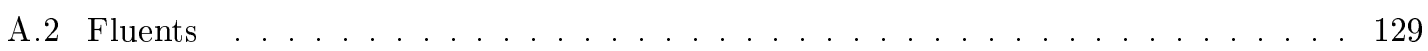

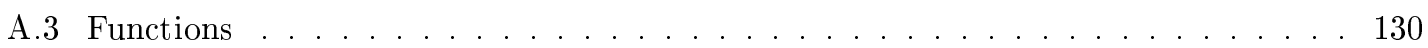

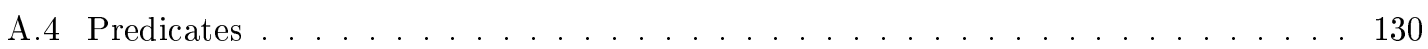

B Notation $\quad 135$

$\begin{array}{lr}\text { Bibliography } & 137\end{array}$

$\begin{array}{lr}\text { Index } & 142\end{array}$ 


\section{Chapter 1}

\section{Introduction and Motivation}

This work presents a novel, formal approach to the so-called symbol anchoring problem, which has been recognized to be crucial in principle for any cognitive robot interacting with the real environment. Our approach provides a general solution to this problem by automatically computing all plausible world models about the objects of the robot's environment, and complies with a broader context of research on artificial intelligence, symbol grounding and philosophy of cognition.

Artificial intelligence research on autonomous agents aims at modelling human intelligent behaviour in order to facilitate cognitive robots solving complex tasks and to improve our understanding of the human mind. Symbolic representations are considered necessary for explaining human intelligence and intelligent behaviour in general [Anderson, 2003b]. Symbols denote objects and states of affairs, this way enabling an agent to maintain an abstract knowledge representation of its world and to reason about symbols. But using symbols also gives rise to the so-called symbol grounding problem: "the problem of how to causally connect an artificial agent with its environment such that the agent's behaviour, as well as the mechanisms, representations, etc., underlying it, can be intrinsic and meaningful to itself, rather than dependent on an external designer or observer" [Ziemke, 1999, p. 1]. In other words, an autonomous agent needs to assign its own semantics to such natural language terms like "table", "sunlight" or "victory" in order to be able to interpret correctly what they stand for. Without its own, intrinsic meaning of symbols, the agent may be simply manipulating meaningless symbolic tokens at best. Typical approaches to the symbol grounding problem presuppose direct interaction with the agent's environment. However, current approaches seem not to have gained considerable approval of the cognitive science community, such that the symbol grounding problem still remains unsolved. Among the reasons for this situation are that the problem is understood differently in various communities such as in psychology or philosophy, who have identified non-uniform requirements regarding an appropriate solution, while some of these requirements seem insurmountable.

A cognitive robot is one which is endowed with animal or human like cognitive capabilities in order to solve complex tasks in complex environments, e.g. the real world. Typical control architectures of cognitive robots include a deliberative level for symbolic knowledge representation and reasoning, possibly a probabilistic level for representing and maintaining the robot's state, and a sensorimotor level for controlling the robot's actuators and interpreting sensor data. ${ }^{1}$ Although data items referring to objects of the world are maintained differently and completely separate on each level of representation, those which stand for the same object of the world have to consistently refer to the very same real-world object regardless of their level of representation in order for the robot to solve its tasks successfully.

For example, consider a cognitive robot servicing in a dynamic household environment and the knowledge of a cup of tea being located on the table in the kitchen. Let some suitable object models on the sensorimotor level afford the robot to recognize certain kinds of objects of interest like a kitchen, a table and a cup. On the one hand, perceptual information about particular

\footnotetext{
${ }^{1}$ Examples of such control architectures of cognitive robots are described in e. g. [Gat, 1992, Althaus and Christensen, 2003, Heintz and Doherty, 2004a].
} 
instances of such kinds of objects is stored at least temporarily in the sensorimotor representation for the purpose of solving the robot's tasks efficiently, e.g. the image region of the cup as seen in the robot's camera. On the other hand, the symbolic reasoning and planning system refers to individual objects using symbols, e.g. $C u p_{1}$. How could we make sure that both data items refer to the very same object of the environment?

The process of creating and maintaining the correct connection between a symbol denoting an object and its corresponding perceptual image (called percept), both referring to the same physical object, is called symbol anchoring. In principle any cognitive robotics system which utilizes symbols denoting objects of the world and perceptual images of these objects has to solve the symbol anchoring problem somehow. While research on symbol grounding considers the meaning of all kinds of symbols, the field of symbol anchoring studies the correspondences between object symbols and their real-world images in particular. Most current cognitive systems implement an ad hoc solution which may work for the specific, intended application under certain conditions. Only since recently a few researchers began to study symbol anchoring systematically towards a domain-independent and well-founded solution. However, we know of no other general and formal approach to the symbol anchoring problem with similarly high expressiveness as the one we present here.

Overview of Results Our work suggests a novel, formal and general symbol anchoring approach with proven characteristics. Based on the first-order logical Fluent Calculus, our approach inherits rich expressiveness with respect to knowledge representation and reasoning. The ability to determine and to maintain all plausible hypotheses of correspondences between object symbols and percepts automatically, counts as one of the outstanding features of our approach. In accordance with phenomenalism, representationalism and the sense-data theory of philosophy of cognition, the computation of our approach is based on a number of fundamental concepts. First, the independence of the symbolic representation from perceptual information has to be retained by symbol anchoring and information fusion. Second, anchoring a certain object symbol has to take into account all potential candidate percepts of the given object category. Conversely, each percept has to be associated with some object symbol as it embodies a potential correspondence candidate. Fourth, intervals of perceptual values endow our approach with a concise, yet explicit representation of perceptual uncertainty, which accounts for sensory noise and imprecision. Lastly, the meaning of perceptual predicates denoting phenomenal concepts of objects like "red", "empty" or "tall", is determined by pre-defined relations with ranges of perceptual values in our approach.

In summary, our work represents a formal and general approach to the symbol anchoring problem, which enhances previous approaches in terms of flexibility, applicability and expressiveness, and which completely automates the process of determining and maintaining all plausible hypotheses of correspondences between object symbols and perceptual images of physical objects. Being applicable in any domain, an implementation of our approach would endow a cognitive robot with a solution to the symbol anchoring problem, which any cognitive robot has to solve in principle. This way, our approach improves the robustness, precision and performance of cognitive robots interacting with and interpreting their environment.

Structure of the Thesis This work comprises two related versions of our symbol anchoring approach. First, the uni-modal version is designed for cognitive robots equipped with a single sensor and is better suited for explaining the basic idea behind our approach. The multi-modal version generalizes from this limitation, while becoming more complex. We also present proofs of important characteristics of our approach, e. g. consistency properties, as well as the proof that the uni-modal approach is a special case of the multi-modal approach. We will compare our approach with other relevant work regarding expressiveness and further important characteristics. As far as it is applicable, we will evaluate our approach according to several prominent criteria and tests known in the literature.

We begin with describing the relevant context of our approach with respect to various fields of research in Chapter 2, motivate and introduce the problem addressed here in detail, and provide 
the foundations that our approach requires. The Chapters 3 to 5 describe our approach in detail and compares it with related work. Thereof, Chapter 3 explicates the fundamental and common concepts of both versions of our symbol anchoring approach. The uni-modal version is explained thoroughly in Chapter 4. Next, Chapter 5 presents our generalized, multi-modal symbol anchoring approach and proves important properties. This work concludes in Chapter 6 with discussions, various evaluations and suggestions for improvements. 


\section{Chapter 2}

\section{Foundations}

The epistemological background of our central topic - the symbol anchoring problem-involves various fields of philosophy. Wittgenstein [1961] proposed a motto that reflects the basic idea behind our approach: "In order to tell whether a picture is true or false we must compare it with reality." In the following, we summarize those philosophical concepts that are the most relevant ones in the context of our approach.

\section{$2.1 \quad$ Philosophy}

This section includes some selected philosophical concepts that we consider important for a better understanding of the motivation behind our approach to the symbol anchoring problem and for explaining the terminology used. Most of the concepts are discussed in the philosophical literature on language or cognition.

\subsubsection{Philosophy of Language}

In general, the research on philosophy of language concentrates on studies about how natural language is used, how language gains its meaning and how speakers interpret language. Being closely related with symbol grounding, philosophy of language also discusses how the meaning of language relates to the real world and to truth. This section will clarify a number of concepts of this field.

\subsubsection{Object}

The concept of an object is probably the most central one in our work, but also one with diverse definitions. A typical, comprehensive dictionary may list more than a dozen entries for the term "object", which range from objects of thought to things that may actually be perceived by the senses. The existence of the object in the real world is emphasized by using the term physical object in our work, similar to Russell [1903] using the term entity. A variable name, e.g. $x$, traditionally stands for an object in logical formulas [Wittgenstein, 1961].

Categories of objects are frequently employed in natural language for abstracting from irrelevant properties or particular entities, while emphasizing that all members of the category share certain characteristics. Nouns, verbs, adjectives and adverbs denote various types of categories: kinds of objects, events, states, features and actions. Each feature of an object and combinations of features represent a potential basis for associating the object with a category. Accordingly, all things are members of an infinite number of different categories. The benefit of categorizing objects is to sort the alternatives that we might confuse the object with, thus reducing the uncertainty involved in acting upon the object according to information theory [Harnad, 2005]. We will make use of such categories of objects in our approach, too, e. g. "book". 


\subsubsection{Reference to Objects}

Various terms for referring to objects are known in the literature on philosophy of language, among which are the proper name, the indexical and the singular proposition.

A proper name is a linguistic or logical expression which refers to a particular object. ${ }^{1}$ While a number of different theories intend to explain the role of proper names in natural language, the descriptive theory sustains our approach at best. The descriptive theory states that the referent of a proper name is determined by a set of properties of the object under consideration. These properties serve as a description of the object, which allows to resolve the reference uniquely in the ideal case [Reimer, 2006].

Similarly, the meaning of a so-called indexical arises from its descriptive content, by which the referent is determined. Examples of indexicals are "I", "now", "he" and "this", which illustrate that the reference to an individual depends on the context of the expression, e.g. time, speaker, hearer and place of the utterance. Hence, the reference is dependent on the meaning which the speaker associates with the expression [Reimer, 2006].

The meaning of a singular proposition can be considered the same as the meaning of an identifier - they both stand for references of particular individuals [Fitch, 2005]. When used as an indexical, singular propositions differ from identifiers. Also note that the referents of constant identifiers are not necessarily unique. For instance, both of the constants "Zeus" and "Jupiter" denote the same individual: the supreme classical deity.

Two kinds of descriptions referring to an object are distinguished in natural language: definite and indefinite descriptions. A definite description determines the reference in a given context uniquely, i.e., the expression denotes a particular individual. ${ }^{2}$ Using Russell's logical formalization of natural language, a definite description is determined by "The $x$ such that $P(x)$ ", where the predicate $P$ denotes the descriptive content of the reference [Russell, 1905]. The strict meaning of the article "the" expresses a definite description in natural language.

In contrast, an indefinite description denotes an arbitrary individual which satisfies the given specification. We typically use the article "a" (or "an") for an indefinite description when we want to express a reference to some suitable object. In Russell's terms, this can be formalized as "An $x$ such that $P(x)$ ", which stands for a reference to an arbitrary member of the class of $P$-objects [Russell, 1905]. Note that an indefinite description indicates a single individual of the given class only.

In general, a symbolic expression is said to designate an object in the cognitive science community if the symbol system can either affect the object directly, or can behave in ways dependent on the object [Newell and Simon, 1976]. The process of determining the referent or referents of a referring expression is called reference resolution in natural language processing. Herein, a pronoun or a definite noun phrase that refers to an object in the world is interpreted based on knowledge about the world and on the context of the given expression [Russell and Norvig, 2003].

\subsubsection{Philosophy of Cognition}

The early example called "Plato's allegory of the cave" illustrates the focus of research in philosophy of cognition nicely [Plato, 360 B.C.]. Plato described how some prisoners are bound in a cave in such a way that they cannot even move or turn their heads, but can only observe the wall in front of them. Their perspective has been restricted like that since their birth. There is a distant fire which casts shadows of the prisoners themselves and of other people in the cave onto the wall. Due to the restricted perspective, the prisoners take the shadows to be actual individuals or objects of the world.

Besides the long debated, pervasive mind-body problem a number of interesting questions arise from Plato's allegory in the field of cognitive robotics. ${ }^{3}$ For instance, could a cognitive robot ever be capable of gaining direct experience of its environment, such that it is aware of its perception,

\footnotetext{
${ }^{1} \mathrm{~A}$ proper name may also purport to refer to an object.

${ }^{2} \mathrm{~A}$ definite description identifies an object uniquely - at least from the perspective of the speaker.

${ }^{3}$ The modern interpretation of the mind-body problem is attributed to Descartes [1965]
} 
and could not be fooled in experiments such as the Total Turing Test (cf. Section 2.2.4.1) [Harnad, 1993]? To what extent does real perception differ from simulated perception of a robot in a virtual world? Modern philosophical theories about these questions seem to be inapplicable to cognitive robotics due to disagreement on foundational issues like consciousness and intelligence of robots.

Two modes of experience are to be distinguished: direct and indirect awareness. To be indirectly aware of something means that the awareness of it depends on the awareness of something else. Accordingly, we perceive an object indirectly, if we perceive it by virtue of perceiving a different object [Jackson, 1977]. Returning to Plato's allegory, the prisoners bound in the cave live with indirect awareness of their environment. Note that indirect awareness is related to, but seems to be independent of consciousness of perception.

Illusion and hallucination are prime examples of perceptual seemings - often introduced in discussions on philosophical theories of cognition-which typify the problem of perception: "If these kinds of error are possible, how can perception be what it intuitively seems to be, a direct and immediate access to reality?" [Steup, 2006] Thus, perception may be characterized according to its immediacy, also called givenness. An experience is called immediate or given, if the cognitive consciousness of it emerges without any inference involved, and if the awareness of it is certain [BonJour, 2001]. Returning again to Plato's allegory of the cave, the immediacy of the prisoners' experience is easy to interpret for an external, omniscient observer, but not for the prisoners themselves.

In the view called direct realism humans are conceived to directly and immediately experience physical objects of the world and any sort of mediation in perception is denied [BonJour, 2001]. The immediacy of perception proposed in this theory eliminates the need for a justifying or causal inference from objects of experience to physical objects of the external world. The naïve realism is a stronger view stating that the appearance of objects of sensory experience is exactly the same as the appearance of physical objects [Le Morvan, 2004]. In contrast, indirect realism asserts that we only experience the world with indirect awareness involved.

Assuming that we only have indirect access to objects in the external world, two theories of the philosophical literature explain how our beliefs are justified by sensory experience: representationalism and phenomenalism. Representationalism holds that the experience of perceived objects forms a subjective representation. The representation is considered to be caused by the physical objects of the external world, thus allowing to infer their existence and justifying our beliefs based on sensory experience [BonJour, 2001]. The subjective representation depicts parts of a larger external world through intermittent and partial perception. It is worthwhile mentioning the distinction between primary qualities of physical objects, e. g. size or shape, and secondary qualities, e. g. colour or taste, a notion which may be attributed to differences in degrees of immediacy of experience. Regarding the above-mentioned allegory of the cave, the representationalist's view allows the prisoners to recognize the physicality of the shadows on the wall, and yet keeping in mind the unlikely event of the shadows being an illusion.

The view called phenomenalism holds that our mental propositions about the external world are solely based on features of and relations between objects of our sensory experience. While this view may appeal to modelling cognitive robots in the first place, it neglects the importance of interaction between an agent and the physical world and of agents communicating features of their surrounding world [Mach, 1914].

What are the sensory objects which we are aware of in our experience of the external world in the representationalism and indirect realism views, and whose shape seems to offer direct access to physical objects intuitively? According to the sense-data theory, which is probably the most popular answer, we are directly aware of private, non-physical entities which constitute our immediate experience. The sense-data objects possess all the phenomenal properties that appear to us through our senses, and thus depend on the particular mind [Huemer, 2005, Crane, 2005].

Furthermore, cognitivism counts among the most influential views, which our approach is based on. Cognitivism relates to representationalism. The former follows the general hypothesis that the central functions of mind and thinking can be modelled by explicit rules manipulating a symbolic representation [Anderson, 2003a]. Cognitive agents and robots are intended to perform complex actions in dynamic environments employing higher-order cognitive abilities like planning, 
reasoning and introspection. The sensory objects abstracting the perception of external, physical objects as parts of the symbolic representation can be considered as internal, subjective and agent dependent sense-data entities.

Perception typically provides a potential source of sensory evidence for the representation of the external world and for the beliefs adopted from the experience. Common sense demands that we should believe what we perceive, and cognitive agents and robots should do likewise. Thus, if the agent's perception is consistent with its beliefs, "the agent should believe what it sees", or in other words, "seeing is normally believing" [Bell and Huang, 1999, p. 1]. The difficulty arises from the fact that beliefs may always be mistaken. The reader is referred to the literature on belief revision and belief update, since these are not the focus of our approach [Hansson, 1997]. Here it suffices to point at the interesting principle of the AGM theory of belief revision that new evidence is to be considered more reliable [Alchourrón, 1982].

\subsubsection{Conclusion}

The theories and views briefly summarized above form the philosophical and conceptual background of our approach. At several stages throughout our below explications, where a conceptual or technological decision was necessary for the approach, we will refer back to these concepts for an appropriate motivation and justification. We intended to minimize the subjective engineering bias of our approach by conforming to established conceptual views wherever the opportunity was given.

\subsection{The Symbol Grounding Problem}

In this work we present a formal, general approach to the symbol anchoring problem. However, before turning to the symbol anchoring problem itself, the more fundamental symbol grounding problem needs to be introduced first.

\subsubsection{Motivation}

The human cognitive and behavioural capacity seems to be symbolic, most notably our natural language and deliberative skills [Harnad, 1990]. The use of symbols in representations is considered necessary for explaining human intelligence and the processes of complex, intelligent behaviour behind it [Anderson, 2003b]. Among typical candidate tasks that are believed to require a symbolic representation are activities involving other agents, because this often causes one to predict their actions, activities reaching beyond the sensory limits of the agent, reasoning about the knowledge of other agents, and solving complex problems [Anderson, 2003a]. The aim of research in artificial intelligence is to develop models of agents which are capable of such complex, intelligent activities. Therefore, especially in the field of so-called Good Old-Fashioned Artificial Intelligence (GOFAI), a term coined by Haugeland [1985], the focus has long been on symbolic information processing models of the mind. Proponents of GOFAI emphasize the importance of symbolic representations; for instance Newell and Simon [1976, p. 114] said, "Symbols lie at the root of intelligent action".

Whenever the tasks a cognitive robot is asked to perform require the system architecture to include a symbolic knowledge representation, the symbol grounding problem arises immediately: How can the symbols of a symbol system bear meaning in itself. For instance, our thoughts are doubtlessly intrinsic to ourselves. On the other hand, the internal representation of a pocket calculator only becomes meaningful in the head of a human interpreter. Hence, the meaning is extrinsic and meaningless to the pocket calculator itself, or in other words, the symbols' "meaning is parasitic on their interpretation through an external observer/user" [Ziemke, 1999, p. 1].

\subsubsection{Description}

Harnad [1990, p. 340] provided a popular description of the symbol grounding problem: "How is symbol meaning to be grounded in something other than just more meaningless symbols?" Ziemke 
[1999, p. 1] defined it more specifically as "the problem of how to causally connect an artificial agent with its environment such that the agent's behaviour, as well as the mechanisms, representations, etc. underlying it, can be intrinsic and meaningful to itself, rather than dependent on an external designer or observer." Most importantly, according to the symbol grounding problem the semantics of symbols cannot be based on just another formal symbol system [Sun, 2000].

Nevertheless, symbolic representations should be associated with semantics, otherwise the operation of a symbol system without intrinsic meaning would result in system failures and would violate its plausibility as a cognitive model [Prem, 1994]. Since early it has been believed that in order for any system to achieve human expert performance in tasks like chess, large stores of semantic information are necessary [Newell and Simon, 1976].

What could constitute an appropriate basis for the meaning of a symbolic representation such that its symbols possess intrinsic meaning? The cognitive science community agreed that, roughly speaking, symbol systems "must be hooked up to the worlds" [Jackson and Sharkey, 1996, p. 174] in order to become meaningful. This means that the internal representation and mechanisms of a cognitive agent ought to have causal connections which allow them to directly interact with their environment, such that no external mechanism mediates the connections. The causal connections and interactions can be realized employing the sensory transducers, e. g. visual or tactile sensors, and effectors of the cognitive robot, e. g. a robotic manipulator arm. Furthermore, internal state variables of the agent could also contribute to direct, causal relations between internal mechanisms and the world [Harnad, 1990, Honavar, 1994]. The physical environment appears to be the most appropriate and precise kind of referent available, which an agent ought to compare its internal picture of the world with. The world also serves as a common foundation for sharing the meaning of internal representations and mechanisms among multiple cognitive robots [McKennoch and Bushnell, 2005].

How could intrinsic meaning of symbols be achieved? The process of associating meaning to symbols of a symbol system in the sense described above is called symbol grounding. Various approaches to the symbol grounding problem have been published so far, but apparently none of them has achieved significant consent among the cognitive science and philosophy communities yet. For instance, the recent, critical review of promising approaches to the symbol grounding problem by Taddeo and Floridi [2005, p. 5] concluded "that symbol grounding is a crucial but still unresolved problem", a conclusion which agrees with the opinion of Cole [2004], Ziemke [1999], Coradeschi and Saffiotti [2003a].

Given that no general approach to the symbol grounding problem has succeeded so far to prove its validity, any symbol system is left in the miserable situation of being ungrounded in principle. ${ }^{4}$ This applies to most current systems for natural language processing because their symbols' meaning is typically based on symbolic descriptions of the world programmed into the system by human designers [Roy, 2004]. The same deficiency can also be identified in symbolic expert knowledge systems, in particular the system Cyc [Lenat, 1995], because the meaning of the symbols is entirely parasitic on their human interpretation [Roy, 2004, Anderson, 2003a].

Harnad [1990, p. 340] pointed at the troublesome reason for symbol systems failing to ground their symbols' meaning: "How is symbol meaning to be grounded in something other than just more meaningless symbols?" This failure is exemplified by typical natural language processing systems and expert knowledge systems, as the meaning of symbols of such systems is typically defined by means of symbolic definitions which in turn use further concept symbols. Such symbol systems utilize rules and symbols, which are syntactic by nature. Symbol meaning, which is defined in terms of nothing but syntactic rules or more concept symbols, fails to ground the meaning in the world due to endless recursive definitions and gets stuck in a "merry-go-round" [Harnad, 1990, p. 340$]$.

The symbol grounding problem turned out to be hard to solve in an appropriate, valid and general way. Accordingly, Vogt [2003, p. 1] considered it "one of the hardest problems in AI and robotics". 5 Nonetheless, the symbol grounding problem remains a crucial problem to be solved,

\footnotetext{
${ }^{4}$ See Section 2.2.4 below for some measures of evaluating the validity of an approach to the symbol grounding problem.

${ }^{5} A I$ is an acronym for Artificial Intelligence, a term supposedly coined by John McCarthy.
} 
because "eventually, any representation must be grounded in something" [Bickhard, 1996].

\subsubsection{Characteristics}

Several characteristics can be recognized as being potential means for evaluating a symbol system with respect to whether it forms a valid solution of the symbol grounding problem. They will be presented next for the sake of clarification as we will refer to them when we describe our approach.

It has been recognized early by René Descartes, that "sensation requires the physicality of the body" [in Anderson, 2003a, p. 93]. This appeals to the notion of embodiment-a property which holds if the considered agent is endowed with a physical body and employs its own sensors and actuators for perception of and interaction with the world [Ziemke, 1999].

The notion of situatedness specifies the individual environmental context an agent is placed in. The context specifies the environmental properties which influence the interaction between the world and the agent, and possibly the agent's experience. From a more general perspective, the context also includes the broad social and cultural environmental characteristics [Anderson, 2003a].

Groundedness positively is the most interesting property of a given symbol system with respect to symbol grounding. It denotes the degree up to which, or the fact whether, a symbol system is capable of grounding its symbols appropriately in the world. However, groundedness is a controversial issue. For instance, Harnad rejected the possibility of various degrees of grounding, and instead regarded groundedness as a binary property of the analysed system [Harnad, 1990, 2001].

The notion of representation learning has reached considerable attention in research on symbol grounding. Ideally, the agent should build its internal symbolic representation on its own from scratch, thus inhibiting any a priori bias of a human designer to influence the representation. Learning the representation would also eliminate any a priori constraints that a given representation may entail [Davidsson, 1993].

There are a number of further characteristics that possibly need to be considered as being important properties of symbol systems with respect to solving the symbol grounding problem, e.g. compositionality, intentionality and systematicity. Due to the disagreement about their relevance to the symbol grounding problem, a discussion is omitted here.

\subsubsection{Evaluation}

This section reviews some criteria that are useful for evaluating a given symbol system with respect to whether it solves the symbol grounding problem appropriately and for the purpose of assessing the symbol system's degree of groundedness.

\subsubsection{The Total Turing Test}

First, we briefly describe the so-called Total Turing Test, which Harnad [1993] conceived for evaluating symbol systems. In addition to the symbolic, behavioural capacity, which a cognitive agent is required to exhibit in order to successfully pass the original Turing Test, the agent has to display the full sensorimotor capacity of humans, in order to pass the Total Turing Test [Turing, 1950]. In particular, Harnad [1993] stated that an agent also needs to be able to "discriminate, recognize, identify, manipulate and describe the objects, events and states of affairs in the world".

According to Harnad [1993], a "grounded system is one that has the robotic and the symbolic capacity to pass the TTT [Total Turing Test] in such a way that its symbols and symbolic activity cohere systematically with its robotic transactions with the objects, events and states of affairs that its symbols are interpretable as being about. In other words, its symbolic capacity is grounded in its robotic capacity rather than being mediated by an outside interpretation projected onto it." Hence, Harnad considers the Total Turing Test an appropriate means for evaluating whether a symbol system solves the symbol grounding problem. This excludes the possibility of various degrees of grounding - instead Harnad regards groundedness as a binary property of the analysed 
system: The considered symbol system either successfully passes the Total Turing Test, or simply fails [Harnad, 1990, 2001].

\subsubsection{Harnad's Test for Grounded Symbol Meaning}

Harnad [1990] proposed another means for evaluating symbol systems, which we will call Harnad's test for grounded symbol meaning subsequently. It consists of two parts. The first, formal part of the test defines a symbol system as

1. "a set of arbitrary physical tokens [...] that are

2. manipulated on the basis of explicit rules that are

3. likewise physical tokens and strings of tokens. The rule-governed symbol-token manipulation is based

4. purely on the shape of the symbol tokens (not their 'meaning'), i.e., it is purely syntactic, and consists of

5. rulefully combining and recombining symbol tokens. There are

6. primitive atomic symbol tokens and

7. composite symbol-token strings. The entire system and all its parts - the atomic tokens, the composite tokens, the syntactic manipulations (both actual and possible) and the rules - are all

8. semantically interpretable: The syntax can be systematically assigned a meaning (e.g. as standing for objects, as describing states of affairs)." [Harnad, 1990, p. 336, our italics]

The second part of the test analyses the symbol system's behavioural capacity, and specifies that the symbol system has to be able to discriminate, categorize and describe all the objects and states of affairs to which its symbols refer. ${ }^{6}$ According to Harnad, the symbols of the symbol system demonstrate to bear intrinsic meaning if the symbol system could pass both parts of the above test successfully [Harnad, 1990].

\subsubsection{The Zero Semantical Commitment Condition}

Taddeo and Floridi [2005] presented a set of very strict criteria for the evaluation of symbol systems with respect to the symbol grounding problem. Their so-called Zero Semantical Commitment Condition, Z-condition for short, agrees with Harnad's statement that the meaning of the symbols of a cognitive agent must be intrinsic to the agent itself [Harnad, 1990]. Hence, the agent has to elaborate its own symbolic representation and its semantics from scratch. Accordingly, the Z-condition specifies that

1. no internal semantic resources must pre-exist in the cognitive agent initially; and

2. no semantic resources must be "uploaded" to the agent; and

3. the agent needs to have its own capacity and resources for developing the semantics of its symbolic representation on its own.

Additionally, Taddeo and Floridi [2005] proposed the following requirements for any symbol system in order to be recognized as a valid solution to the symbol grounding problem, which we will denote Taddeo and Floridi's symbol grounding requirements subsequently. Accordingly, a symbol system is required to comprise:

\footnotetext{
${ }^{6}$ Note that Harnad's term "to identify" stands for what we typically mean by "to categorize". We use the much more common term in order to avoid confusion.
} 
1. a sensorimotor process for bottom-up grounding;

2. a top-down feedback process "that allows the harmonization of top level grounded symbols and bottom level, sensorimotor interactions with the environment";

3. the capacity of the agent to develop its own representation;

4. the capacity of the agent to abstract, i. e., to categorize;

5. the capacity of the agent to share and synchronize its own symbol meaning (grounding) by means of communicating with other agents;

6. "an evolutionary approach in the development of (1)-(5)";

7. "the satisfaction of the Z-condition in the development of (1)-(6)." [Taddeo and Floridi, 2005, p. 35]

Unfortunately, none of the eight prominent systems reviewed in [Taddeo and Floridi, 2005] succeeded in solving the symbol grounding problem in accordance with the above-mentioned criteria. The authors of the criteria were not sure either, whether any system possibly could fulfil all these conditions in principle. Therefore, the practical relevance of these evaluation criteria may be questionable, but they seem to be reasonable and desirable.

\subsubsection{Conclusion}

Our tentative conclusions regarding important characteristics of symbol systems are as follows. In order for a cognitive agent to approach the symbol grounding problem, it is required to have a physical body situated in the world. Furthermore, it has to be equipped with sensors and actuators which provide direct interaction with the world. Such a suitable kind of agent should be more specifically called an embodied, cognitive robot. Of course, the agent employs a symbol system for knowledge representation, because the symbol grounding problem would be pointless for this agent otherwise. Furthermore, symbol grounding seems to require the agent's internal representation to adapt to the constraints which the interaction with the world compels.

The above-mentioned criteria for evaluating symbol systems with respect to the symbol grounding problem will be used as a guideline for evaluating our own approach to the symbol anchoring problem, too. The aims of our approach go far beyond solving the symbol anchoring problem alone-our approach addresses certain aspects of the symbol grounding problem additionally. Therefore, we placed our approach in the right, broader context of the symbol grounding problem and philosophy of cognition from the beginning on.

\subsection{The Symbol Anchoring Problem}

This section describes the central topic of our work, the so-called symbol anchoring problem. After specifying this problem, you will learn about the difficulties and requirements involved in approaching it. Thereafter, the subsequent chapters will demonstrate how our approach intends to solve the symbol anchoring problem.

\subsubsection{Motivation and Description}

Cognitive robots are typically situated in the real world and include a deliberative component besides other modules, which employs a symbolic representation of the world, sensors and actuators, as well as sensorimotor processes for controlling the sensors and actuators. ${ }^{7}$ The deliberative

\footnotetext{
${ }^{7}$ The symbol anchoring problem also applies to cognitive agents in virtual worlds in principle, and our approach presented below should be applicable in this kind of domain, too. Anyway, this approach focuses on real worlds, because real worlds offer the full extent of potential interactions between the agent and its environment. Moreover, this approach takes advantage of the strong relation between the symbol anchoring problem and the symbol grounding problem. Since the symbol grounding problem presupposes the real world, we address cognitive robots acting in the real world.
} 


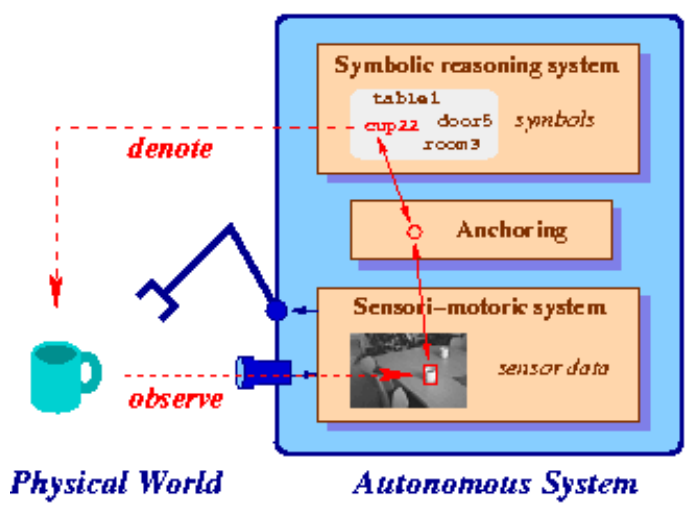

Figure 2.1: Symbol anchoring in a typical architecture of a cognitive robot.

process abstracts from the high bandwidth of perceptual data flow in order to facilitate abstract reasoning about the world using a symbolic representation. Using constant and variable symbols denoting objects, individuals and other robots in the world affords the deliberative process to reason about them, to plan actions with them and to communicate propositions about them to other robots or humans. Sensorimotor processes, in contrast to deliberative processes, typically utilize low-level representations of entities of interest in terms of perceptual images. Hence, the individual representations of the deliberative and sensorimotor processes of a cognitive robot are very different, just as the processes are different themselves. The detachment of deliberative and sensorimotor representations give rise to the so-called symbol anchoring problem, because both an object symbol of the deliberative representation and its corresponding perceptual data item separately and independently refer to the same object of the world (cf. Figure $2.1^{8}$ ). The process of creating and maintaining the correct connections between symbols denoting objects and their corresponding perceptual images, both of them referring to the same physical objects, is called symbol anchoring [Broxvall et al., 2004b]. ${ }^{9}$

The symbol anchoring problem is more specific than the symbol grounding problem. Symbol grounding deals with symbols denoting objects, events and states of affairs of the world and also includes more abstract symbols denoting concepts. In contrast to symbol anchoring, symbol grounding seeks to found the meaning of symbols directly on the relation with the physical world. Some people roughly distinguish the two research topics by associating symbol anchoring with the engineering aspects of building a practical, cognitive robot on the one hand, and by associating symbol grounding with the philosophical issues of symbol meaning in relation to the real world on the other hand [Vogt, 2003, Prem, 2002].

The task of symbol anchoring requires the following basic properties of a cognitive agent. Naturally, the symbol anchoring problem applies only if the agent utilizes symbols denoting objects in the world. The agent needs to have its own physical body situated in the world, sensors for perception and actuators for interaction. Thus, the kind of agent which the symbol anchoring problem addresses should be called embodied cognitive robot. These basic requirements match those of agents approaching the symbol grounding problem, as has been described in Section 2.2.5 above.

The symbol anchoring community has recognized that a general solution to the symbol anchoring problem is very hard to find [Coradeschi and Saffiotti, 2003a]. Blank et al. [2002] even considered the symbol anchoring problem to be AI-complete. In general, a problem is called to be AI-complete, if solving this problem is equivalent to solving the entire AI problem, i. e. "producing a generally intelligent computer program" [Shapiro, 2000, p. 6].

Nevertheless, cognitive agents need to establish and to maintain the correct correspondences between object symbols, which they reason about and plan with, and their environment in order

\footnotetext{
${ }^{8}$ Courtesy of Alessandro Saffiotti [Coradeschi et al., 2001].

${ }^{9}$ Symbol anchoring is also called perceptual anchoring sometimes.
} 
to be able to accomplish their tasks successfully [Coradeschi et al., 2001]. This necessity especially becomes apparent in such applications which involve multiple cognitive robots and interaction with humans, e.g. cognitive service robots for office or household environments, where the robots have to communicate about propositions on objects in the environment, because they have to be able to resolve the referent objects correctly [Bredeche et al., 2003].

Although any cognitive robot with characteristics as described above has to solve the symbol anchoring problem in principle, a general study thereof has not developed until recently [Coradeschi and Saffiotti, 2002]. Instead of implementing a general theory, the symbol anchoring approaches of many previous cognitive robots were tailored to, and thus typically limited to, the specific application domain they were designed for. The concepts of symbol anchoring of such approaches were hidden in the code and the actual problem was solved in an ad hoc manner [Coradeschi and Saffiotti, 2003a]. Today's approaches to the symbol anchoring problem can be considered to provide a practical approach, but with limited generality and functionality. This situation may be due to the youth of research on hybrid systems in general [Anderson, 2003a]. Proponents of research on symbol anchoring like Coradeschi and Saffiotti [2002] and ourselves advocate a general, domainindependent theory because it simplifies the reuse of symbol anchoring technologies for future cognitive systems, broadens the applicability of an approach, and extends and optimizes their functionality. First and foremost, we aim at a systematic solution which provides the consistency and robustness required by today's real-world cognitive robots.

\subsubsection{Terminology}

Throughout this work at hand, a few terms denoting basic concepts are used whose meaning is outlined here in order to enhance readability. In accordance with the phenomenalism of cognition, physical objects in the environment of a cognitive robot can be measured in virtue of their sensory features (cf. p. 7). Those sensory features that can be directly measured are called perceptual attributes..$^{10}$

In general, robotic sensors perceiving physical objects yield multiple perceptual attributes, even if only a single sensing device is in use. A structured collection of such sensory measurements is a so-called percept. Most importantly, all measurements of a percept are assumed to originate from the same physical object. ${ }^{11}$

The main task of a symbol anchoring system is to maintain the correspondences of multiple objects over time. The internal representation of a correspondence between an object symbol and the object's percept is called anchor.

\subsubsection{Characteristics}

Typically, symbol anchoring approaches follow a certain direction with respect to the symbolic and sensorimotor representations: bottom-up, top-down or in both directions in parallel. Hereof a bottom-up direction of symbol anchoring stands for a process, which tries to determine the corresponding object symbol given a percept of the sensorimotor representation. Conversely, a top-down symbol anchoring approach tries to figure out the corresponding percept given a certain object symbol. The symbol anchoring process has not necessarily to be designed in a single direction only. Actually, the research community has recognized that symbol anchoring should be performed in both directions in parallel in order to achieve the required functionality completely [Bell et al., 2002].

The following practical challenges constitute aims of approaches to the symbol anchoring problem. An approach seeking most general expressiveness and full functionality ought to accommodate all of these challenges. This list also serves as a means to evaluate a given symbol anchoring approach, although it is certainly not exhaustive and further interesting features of symbol anchoring could be conceived.

\footnotetext{
${ }^{10}$ This definition conforms to the one of [Bell and Huang, 1999].

${ }^{11}$ This notion of a percept agrees with the one of [Coradeschi and Saffiotti, 2003a].
} 


\subsubsection{Uncertainty}

Limited precision and reliability, and the influence of noise on perception are natural sources of perceptual uncertainty inherent in practical sensor systems that cannot be eliminated completely. Every symbol anchoring approach has to take perceptual uncertainty into account. This requires an appropriate representation as well as computation process. A (low-level) object recognition system that pre-processes the perceptual measurements with respect to filtering out noise in the first place constitutes a valuable service, for instance.

If the uncertainty of perceptual information about physical objects is rather high, then this situation gives rise to multiple physical objects which may potentially correspond with a certain object symbol, but the correct correspondence cannot be determined directly. The ambiguity of correspondences in such situations has to be resolved sooner or later, e. g. using further perceptual cues, interacting with the object of interest or communicating with other agents.

\subsubsection{Incomplete Knowledge}

The limitation of equipment of a typical cognitive robot to a certain variety and number of sensors, the sensors' limited precision and environmental aspects like distance to or obstruction of the object of interest give rise to limited perceptual information available to the robot, to name just a few practical hurdles. The robot's symbolic (qualitative) knowledge about objects in its environment is incomplete in any given situation in general. Altogether, these real-world circumstances induce ambiguity of correspondences between symbols and percepts, which may potentially prevent the robot from performing its tasks successfully. Hence, the symbol anchoring approach has to take incompleteness of knowledge into account.

\subsubsection{Vagueness of Information}

Cognitive robots - as considered in our approach-work with propositions about physical objects and properties thereof at the symbolic level. Such propositions and properties are used in definite and indefinite descriptions for specifying the objects of interest. A linguistic term used for specifying a certain property of an object should be considered as a summary of sensory information, while each linguistic term bears its individual degree of specificity and detail, which depend on the context, too [Roy and Reiter, 2005]. A symbol anchoring approach has to take the individual vagueness into account in order to be able to associate the given description of an object with percepts correctly.

\subsubsection{Invariance of Perceptual Information}

There are two different perspectives, which the perceptual information can be based on in principle. Let us call subjective perception the perspective, where the measured value of a perceptual attribute is taken as is from the sensing system without much conversion. Subjective perception ignores environmental influences on the appearance of perceptual attributes such as sunlight brightening the colours of objects.

In contrast, an advanced perception system takes environmental influences and the robot's circumstances into account by transforming its measurements into a much more objective frame of reference. This transformation process may require considerable contextual information and knowledge about the physics of the robot's sensors. However, employing objective perception would especially pay off in dynamic environments and application domains with mobile robots, where the dependency of perception on environmental factors is rather high. A common scale of perception is also necessary if multiple heterogeneous robots exchange perceptual information on objects in shared environments.

The following example will illustrate the advantages of objective perception. Imagine a cognitive robot measuring the height of an object using its camera. Perceiving the object from some distance yielded its height in terms of a certain number of pixels; but this value changes dramatically when approaching the object. In contrast, objective perception means to convert this 
measurement into an absolute height of the object in terms of comparable world coordinates, which can be maintained and communicated easily and consistently.

\subsubsection{Composite Objects}

The definition of a percept has been deliberately chosen such that the percept covers the measured physical object completely, and only this object (cf. Section 2.3.2). Particularly, a percept must not characterize components of an object only, nor cover multiple different objects at once. The reason for this definition is that anchoring an object symbol to a physical object addresses the object as a whole based on the object's functional role. However, typical real-world applications also include large or complex objects which a robot's sensor cannot recognize easily. For instance, a two-dimensional laser scanner operating on a low horizontal plane can only perceive parts of the humans' legs, which certainly complicates robust recognition of a human as a whole. We share this definition of a percept with for instance Kleinehagenbrock et al. [2002] and Lang et al. [2003] as it appears to be advantageous for a symbol anchoring approach.

The correct comprehension of perceptual information about complex objects requires domainspecific knowledge on spatial relations between individual parts or features of these objects and probably the employment of multiple sensors of various modalities. While our work presents a symbol anchoring approach for multi-modal perception in Chapter 5, anchoring of composite objects is not the focus here.

\subsubsection{Reference to Objects}

To determine and to maintain references to objects in the robot's environment is the central topic of symbol anchoring for cognitive robots. Various kinds of references to objects are known in general, e. g. definite references (also called definite description), indefinite references (likewise, also called indefinite description) and proper names (cf. Section 2.1.1.2). Besides others, perceptual attributes (e.g. the colour red, the person being tall etc.) and functional properties of the objects (e.g. something to hold water) facilitate to determine and to verify the appropriateness of individual correspondences between object symbols and percepts. It seems that a general theory of symbol anchoring - as advocated by ourselves and others - shall offer all kinds of references to objects in order to provide the richest expressiveness to the symbolic reasoning system. Moreover, a symbol anchoring approach ought to utilize all means of determining and verifying the correspondences in order to maximize the certainty in their correctness (cf. Section 2.3.1).

\subsubsection{Conclusion}

An intelligent symbol anchoring strategy should be able to resolve ambiguous situations with respect to correspondences between symbols and percepts to the extent necessary for successful completion of the robot's tasks. A symbol anchoring approach should also take uncertainty, incompleteness and vagueness of symbolic and perceptual information into account, because it appears almost impossible to determine correct correspondences between symbols and physical objects otherwise. It seems to be very helpful if the cognitive robot knows its own sensory capacity and employs active perception, i.e., the capacity to plan with and to reason about sensor actions and sensor information. We believe that a good strategy for symbol anchoring with uncertain and incomplete perceptual information is to make use of all perceptual and symbolic information available, to know the limits of this information, to drive goal-directed knowledge acquisition and to analyse the whole situation by reasoning about its constituents. Our approach is intended to accommodate all these goals.

\subsubsection{Evaluation}

For comparing different approaches to the symbol anchoring problem regarding their respective functionality a general set of characteristics is needed, which is not specific to individual approaches. Unfortunately, the symbol anchoring community has neither proposed a multitude of 
such evaluation criteria nor agreed on a common set thereof yet. Coradeschi and Saffiotti [2002] provided the most useful list of general evaluation criteria up to our knowledge and time of writing. They name the following functional requirements for any approach to the symbol anchoring problem to be useful:

- The approach should facilitate that the reference to an object may be specified in form of a symbolic description, i. e., a list of perceptual attributes and expected values.

- The approach should facilitate the search for a requested object in the world. More precisely, the task is to establish a non-existent correspondence with a physical object. This requires the approach to match the symbolic information of the object under consideration with percepts received from the object recognition system.

- The approach should be able to deal with ambiguous situations appropriately. Ambiguity should be resolved if possible.

- If continuous perceptual information is provided by the object recognition system of the same physical object over some period of time, the symbol anchoring approach should track and update its correspondence.

- Upon failure to track an object's correspondence, the approach should be able to reacquire the correspondence if the correct physical object reappears among the new percepts [Coradeschi and Saffiotti, 2002].

We agree on the usefulness of these features, and thus considered them as the most important functionalities of our symbol anchoring approach from the beginning.

\subsubsection{Previous Works}

This section summarizes some previous works on the symbol anchoring problem, which are noteworthy with respect to their expressiveness, generality and for a comparison with our approach. In our opinion, some of the most considerable formal approaches have been published by Alessandro Saffiotti, Silvia Coradeschi and their colleagues at the University of Örebro, Sweden. Let's divide the various, published computational approaches to the symbol anchoring problem of this group into a basic approach, an approach based on Fuzzy logic and another approach based on conceptual spaces. The following summaries of the three approaches refer to a recent publication on each version according to our knowledge and time of writing. However, there exist a number of improved versions in later papers, too. We will refer to some of them in our conclusion sections.

\subsubsection{The Basic Approach of Saffiotti et al.}

The computational theory for symbol anchoring as described in [Coradeschi and Saffiotti, 2001] comprises a symbol system and a perceptual system. The symbol system includes a set of symbols (variables or constants), $\mathcal{X}=\left\{x_{1}, x_{2}, \ldots\right\}$, denoting individual objects of the environment, and a set of predicates, $\mathcal{P}=\left\{p_{1}, p_{2}, \ldots\right\}$. The perceptual system includes a set of percepts, $\Pi=\left\{\pi_{1}, \pi_{2}, \ldots\right\}$, where the meaning of a percept is defined as in Section 2.1.2. The individual percepts $\pi_{i}$ are basically identifiers, likewise the symbols $x_{i}$. The set of perceptual attributes $\Phi=\left\{\phi_{1}, \phi_{2}, \ldots\right\}$ names the properties of physical objects, which the cognitive agent can measure (cf. Section 2.3.2). The attributes take values in their respective domains $D_{i}$.

A so-called predicate grounding relation establishes the correspondences between unary predicates denoting perceptual properties and perceptual values. This relation is used to ground a symbolic description by mapping each predicate to a range of corresponding perceptual values depending on a perceptual attribute. Given $D \stackrel{\text { def }}{=} \bigcup_{i} D_{i}$ the predicate grounding relation is defined as

$$
g \subseteq \mathcal{P} \times \Phi \times D
$$


A symbolic description $\sigma \in 2^{\mathcal{P}}$ is a collection of properties and allows to specify objects of the environment by means of perceptual predicates for the purpose of defining a basic definite reference. The symbolic description of an object is given in a typical symbol anchoring task or can be easily extracted from the agent's symbolic knowledge about the object in question. Conversely, the socalled perceptual signature represents the values of a given percept measured for certain perceptual attributes and is defined as a partial function $\gamma: \Phi \mapsto D$. Because not all perceptual attributes are measured of each object in general, this function can only be defined partially, where feat $(\gamma)$ enumerates those attributes that are actually available of a given percept.

The task of symbol anchoring in this setting is to determine the correct correspondence (or multiple possible correspondences) between a symbolic description $\sigma$ and the percepts $\Pi$ currently measured. To this end Coradeschi and Saffiotti [2000] suggested the predicate "match" with

$$
\operatorname{match}(\sigma, \gamma) \Leftrightarrow \forall p \in \sigma . \exists \phi \in \operatorname{feat}(\gamma) . g(p, \phi, \gamma(\phi))
$$

for example. Generally speaking, this predicate ought to be true for those correspondences, where the symbolic and perceptual information agree. In particular, this definition verifies whether the ranges of perceptual values associated with the symbolic predicates of the symbolic description coincide with the perceptual values measured of the percept considered.

The description state $D S_{t}: \mathcal{X} \mapsto 2^{\mathcal{P}}$ is used to associate each object identifier with a symbolic description depending on time $t \in T$. Conversely, the perceptual state $P S_{t}: \Pi \times \Phi \mapsto D$ associates each percept identifier with a perceptual signature at time $t .{ }^{12}$ The perceptual state is undefined for those percepts which are not perceived at time $t$. The set $V_{t}$ contains those percepts which are actually perceived at time $t$.

The correspondences between an object symbol and a percept are represented as an anchor and defined as the predicate $\alpha$ with ${ }^{13}$

$$
\alpha: T \times \mathcal{X} \times \Pi \times \Phi \times D \times 2^{\mathcal{P}}
$$

According to this definition, the anchor representation consists of four components; an object identifier $\alpha_{t}^{\mathrm{sym}}$, a percept (identifier) $\alpha_{t}^{\mathrm{per}}$, a perceptual signature $\alpha_{t}^{\mathrm{sig}}$ and a storage $\alpha_{t}^{\mathrm{obs}}$ for those predicates of the symbolic description that have been observed. The component $\alpha_{t}^{\text {sig }}$ represents the best estimate of perceptual information about the considered object measured so far, i. e., it is incrementally refined over time and continues to hold the best estimate even when no percept is associated.

In [Coradeschi and Saffiotti, 2001, p. 409] an anchor is said to be "grounded at time $t$ iff $\alpha_{t}^{\text {per }} \in V_{t}$ ". In other words, for an anchor to be grounded it is sufficient that some percept is associated with the anchor. Note that the authors proposed a stricter version of groundedness previously, where match $\left(D S_{t}\left(\alpha_{t}^{\mathrm{sym}}\right), P S_{t}\left(\alpha_{t}^{\mathrm{per}}\right)\right)$ had to be true for the anchor in question, too [Coradeschi and Saffiotti, 2000, p. 131]. This means for an anchor to be grounded, that the percept has to be among the current percepts measured, and the symbolic description for the object symbol has to agree with the current perceptual estimate.

Of course the aim of symbol anchoring is to establish a grounded correspondence between an object symbol and a percept. But of similar importance is that the correspondence is correct. According to [Coradeschi and Saffiotti, 2001, p. 409] "an anchor $\alpha$ is referentially correct if, whenever $\alpha$ is grounded at $t$, then the physical object denoted by $\alpha_{t}^{\mathrm{sym}}$ is the same as the one that generates the perception $\alpha_{t}^{\text {per }}$." Coradeschi and Saffiotti [2001, p. 409] defined the symbol anchoring problem as "the problem to find referentially correct anchors."

The considered symbol anchoring approach employs the function "match", which verifies whether the given perceptual signature is consistent with the given symbolic descriptor for those attributes which have been measured. They extended the definition of function "match" of Formula (2.1) as follows:

\footnotetext{
${ }^{12}$ This is how the original definition $P S_{t}: \Pi \mapsto(\Phi \mapsto D)$ shall be interpreted.

${ }^{13}$ The original definition was $\alpha: T \mapsto \mathcal{X} \times \Pi \times(\Phi \mapsto D) \times 2^{\mathcal{P}}$ which we interpret in the above, formally correct way.
} 


$$
\begin{gathered}
\operatorname{match}(\sigma, \gamma)= \begin{cases}\emptyset & \text { if } \exists p \in \sigma \cdot(\operatorname{obs}(p, \gamma) \wedge \neg \operatorname{cons}(p, \gamma)) \\
\{p \in \sigma \mid \operatorname{obs}(p, \gamma)\} & \text { otherwise }\end{cases} \\
\operatorname{obs}(p, \gamma) \Leftrightarrow \exists \phi \in \operatorname{feat}(\gamma) \cdot \exists d \in D \cdot g(p, \phi, d) \\
\operatorname{cons}(p, \gamma) \Leftrightarrow \exists \phi \in \operatorname{feat}(\gamma) . g(p, \phi, \gamma(\phi))
\end{gathered}
$$

Hereof predicate $\operatorname{obs}(p, \gamma)$ is true if the symbolic property $p$ has been perceived according to the perceptual signature $\gamma$, while in addition predicate $\operatorname{cons}(p, \gamma)$ holds if the perceived value agrees with the symbolic property $p$. The function "match" returns those symbolic properties that have been verified by the perceptual information $\gamma$, or $\emptyset$ if an inconsistency occurred.

The approach of Coradeschi and Saffiotti [2001] discussed above also includes three algorithms called symbol anchoring functionalities, that manipulate the anchor representation. The functionality "Find" creates an anchor and grounds a given symbol if the object is perceived for the first time. The functionality "Reacquire" is supposed to restore a previously grounded correspondence for a given symbol. Lastly, "Track" is supposed to update the anchor of a given symbol with a new percept. The reader is referred to the original publications cited above for further details.

\subsubsection{The Fuzzy Logic Approach of Saffiotti et al.}

This section briefly summarizes the Fuzzy logic approach of Saffiotti and colleagues published in [Coradeschi and Saffiotti, 1999, Coradeschi et al., 2001]. This approach is intended to improve the method of handling perceptual uncertainty. Similar to the computational theory of the basic approach described in the above Section 2.3.5.1, their Fuzzy logic approach includes a symbol system that comprises a set of symbols $\mathcal{X}=\left\{x_{1}, x_{2}, \ldots\right\}$ and a set of perceptual predicates $\mathcal{P}=\left\{p_{1}, p_{2}, \ldots\right\}$ on the one hand, as well as a perceptual system including a set of percepts $\Pi=\left\{\pi_{1}, \pi_{2}, \ldots\right\}$ and a set of perceptual attributes $\Phi=\left\{\phi_{1}, \phi_{2}, \ldots\right\}$ on the other hand. ${ }^{14}$ The Fuzzy predicate grounding function $g: \mathcal{P} \times \Phi \mapsto F_{\phi}$ defines the meaning of the perceptual predicates in terms of Fuzzy sets $F_{\phi}$. A perceptual predicate stands for a linguistic variable in Fuzzy logic terms. The predicate grounding function depends on the attribute considered; e.g., $g$ (smallsuitcase, width $)=$ SMALL $_{\mathrm{W}}$ relates the abstract concept of a suitcase having a small width to an appropriate Fuzzy set. The measured sensory data of an object of the environment are represented in the perceptual signature $\gamma: \Phi \mapsto \operatorname{Dom}(\Phi)$, which associates perceptual values within the domain $\operatorname{Dom}(\Phi)$ with attributes for a given percept. Though the authors suggested to represent perceptual uncertainty by a possibility distribution, they actually used plain intervals [Coradeschi et al., 2001, p. 112].

The degree of matching a symbolic description $\sigma \in 2^{\mathcal{P}}$ with the measured data of a certain percept $\Gamma$ is computed in two steps. First, for each attribute $\phi$ corresponding to predicate $p \in \sigma$ by the function "match" with

$$
\operatorname{match}\left(F_{\phi}^{i}, \gamma(\phi)\right):=\left\langle\operatorname{Poss}\left(\gamma(\phi) ; F_{\phi}^{i}\right), \operatorname{Nec}\left(\gamma(\phi) ; F_{\phi}^{i}\right)\right\rangle
$$

which is based on the Fuzzy logical function "Poss", which in turn computes the degree of necessity using the supremum minimum. The function "Nec" represents the second parameter, which returns the degree of possibility using the infimum maximum. Finally, the overall degree of matching is obtained from the individual matching degrees using a T-norm operator.

The other components of the computational framework resemble those of the basic approach summarized above in Section 2.3.5.1. Only an abstract, verbal description is available in [Coradeschi et al., 2001, p. 113] on what the symbol anchoring functionalities shall provide, without details on their definition or implementation.

\footnotetext{
${ }^{14}$ We adapted some formulas of this section for the sake of formal correctness and readability.
} 


\subsubsection{The Conceptual Spaces Approach of Saffiotti et al.}

Chella et al. [2004] presented an extension of the basic symbol anchoring approach of Coradeschi and Saffiotti [2001], where they introduced an intermediate representation in between the symbolic and perceptual representations. In particular they suggested to use so-called conceptual spaces, brought forward by Gärdenfors [2000], as the appropriate intermediate representation, because it should allow to map both symbolic and perceptual information into a single representation.

Briefly speaking, a conceptual space is a multi-dimensional, metric representation which facilitates to define a similarity measure based on a metric distance between two entities. An entity of the conceptual space is called a knoxel. At any point of time, this approach represents an object of the world by a dynamic knoxel in the conceptual space. Formally, a conceptual space $K$ consists of $m$ dimensions each having their respective domain $K_{1}, \ldots, K_{m}$. The sensor model associates each measurement with a knoxel, $h: V \mapsto 2^{K}$, where $v_{t} \in V$ denotes an individual measurement. ${ }^{15}$ Abstract, perceptual predicates denoting concepts are mapped to partial spaces using the predicate grounding function $g: \mathcal{P} \mapsto 2^{K}$, this way grounding the predicates' meaning. The mapping of a symbolic descriptor $\sigma \in 2^{\mathcal{P}}$ is determined by its constituents according to $g(\sigma)=\bigcap_{p \in \sigma} g(p)$. The anchoring function $\alpha: T \mapsto \mathcal{X} \times K \times K$ embodies the intermediate representation for object symbols $x \in \mathcal{X}$ depending on time $t \in T$ such that an anchor structure $\alpha(t)=\langle x, k, p\rangle$ associates the object $x$ with the current estimate $k$ of perceptual information about this object, and with the percept $p$.

The purpose of the anchoring functionality "Find" is basically to anchor a given object symbol to the best matching, current percept. Accordingly, it is executed on demand, in a top-down fashion. The functionality "Acquire" simply creates the data structure "anchor" for a new percept of interest. Which symbol is assigned to the new anchor is undefined. If a symbol is already associated with a percept via some anchor structure, the functionality "Track" is supposed to carry the anchor forward from the previous time of perception. First, it predicts the perceptual signature, and then it updates the signature given a matching, new percept. The functionality "Reacquire" selects among the current percepts the one which matches the symbolic description of the requested anchor best, and updates the perceptual signature of the anchor according to this percept. The reader is referred to the original publication for further details (cf. [Chella et al., 2004]).

\subsection{Basics of the Fluent Calculus}

For those of the audience not familiar with the Fluent Calculus, we need to introduce this logical calculus, since our approach is based on it. This section summarizes the basics of the Fluent Calculus language, in particular the most unified version of the Fluent Calculus described in detail in [Thielscher, 2005]. ${ }^{16}$

The Fluent Calculus is a many-sorted first-order logic language with equality, extending the classical Situation Calculus with the concept of a state [McCarthy, 1963]. By using the Fluent Calculus as the foundational logical language, our symbol anchoring approach benefits from its capacity. This calculus allows to model incomplete knowledge, non-deterministic actions, indirect effects of actions, unexpected failures of actions, noisy perception and imprecise effectors for cognitive agents performing in real, dynamic worlds. The Fluent Calculus provides solutions to the classical frame problem [McCarthy and Hayes, 1969], the ramification problem [Ginsberg and Smith, 1988] and the qualification problem [McCarthy, 1977].

Information about the state of the environment is modelled using fluents and states. Fluents are terms of the fundamental sort FLUENT, while states are terms of sort STATE. States are made

\footnotetext{
${ }^{15}$ We suggest an alternative definition of function $h$ for this approach, which makes much more sense for anchoring multiple objects: $h: V \times T \mapsto 2^{K}$.

${ }^{16}$ This summary is not meant to be an exhaustive, in-depth account of the Fluent Calculus, but provides an abstract outline of those parts relevant to our approach. The reader is referred to [Thielscher, 2005] for further details.
} 
up of fluents using the associative and commutative connection function $\circ$ with

$$
\circ: \text { STATE } \times \text { STATE } \longmapsto \text { STATE }
$$

The function $\circ$ computes the union of two states and is written in infix notation. The special constant $\emptyset$ : STATE denotes the empty state. The Fluent Calculus employs an equation for expressing whether a certain property holds in the given state, which is abbreviated by the following macro:

$$
\text { Holds }(f \text { : FLUENT, } z \text { : STATE }) \stackrel{\text { def }}{=}\left(\exists z^{\prime}\right)\left(z=f \circ z^{\prime}\right)
$$

The foundational axioms $\Sigma_{\text {state }}$ of the Fluent Calculus are:

1. Associativity and commutativity: $\left(z_{1} \circ z_{2}\right) \circ z_{3}=z_{1} \circ\left(z_{2} \circ z_{3}\right)$

$$
z_{1} \circ z_{2} \quad=\quad z_{2} \circ z_{1}
$$

2. Empty state axiom: $\neg$ Holds $(f, \emptyset)$

3. Irreducibility: $\operatorname{Holds}(f, g) \supset f=g$

4. Decomposition: $\operatorname{Holds}\left(f, z_{1} \circ z_{2}\right) \supset \operatorname{Holds}\left(f, z_{1}\right) \vee \operatorname{Holds}\left(f, z_{2}\right)$

5. State equality: $(\forall f)\left(\operatorname{Holds}\left(f, z_{1}\right) \equiv \operatorname{Holds}\left(f, z_{2}\right)\right) \supset z_{1}=z_{2}$

The removal of fluents from states is defined in accordance with a valid solution to the frame problem as follows.

$$
\begin{array}{rll}
z_{1}-\emptyset=z_{2} & \stackrel{\text { def }}{=} z_{2}=z_{1} \\
z_{1}-f=z_{2} & \stackrel{\text { def }}{=}\left(z_{2}=z_{1} \vee z_{2} \circ f=z_{1}\right) \wedge \neg \operatorname{Holds}\left(f, z_{2}\right) \\
z_{1}-\left(f_{1} \circ f_{2} \circ \cdots \circ f_{n}\right)=z_{2} & \stackrel{\text { def }}{=}(\exists z)\left(z_{1}-f_{1}=z \wedge z-\left(f_{2} \circ \cdots \circ f_{n}\right)=z_{2}\right)
\end{array}
$$

The addition of fluents to a state, $z_{1}+f_{1} \circ \cdots \circ f_{n}=z_{2}$, is defined as an abbreviation for the formula $z_{1} \circ f_{1} \circ \cdots \circ f_{n}=z_{2}$. Combining both addition and removal of fluents into the following state update equation shows how the Fluent Calculus solves the frame problem:

$$
z_{2}=z_{1}-f_{1} \circ \cdots \circ f_{m}+g_{1} \circ \cdots \circ g_{n} \stackrel{\text { def }}{=}(\exists z)\left(z_{1}-f_{1} \circ \cdots \circ f_{m}=z \wedge z_{2}=z \circ g_{1} \circ \cdots \circ g_{n}\right)
$$

The sort ACTION includes terms denoting the agent's actions. The sort SIT comprises situation terms each representing sequences of actions. The initial situation is called $S_{0}:$ SIT until which no actions were performed. The standard function $D o$ maps a situation to its successor situation given the action performed and is defined as:

$$
\text { Do }: \text { ACTION } \times \text { SIT } \mapsto \mathrm{SIT}
$$

The function State $:$ SIT $\mapsto$ STATE determines the relation between a certain point in the sequence of action execution and the model of the environment at this stage. This function allows to define, when a property (i. e. a fluent) holds in a given situation: $\operatorname{Holds}(f, s) \stackrel{\text { def }}{=} \operatorname{Holds}(f, \operatorname{State}(s))$.

The following two kinds of formulas are part of subsequent definitions. "A state formula $\Delta(z)$ is a first-order formula with free state variable $z$ and without any occurrence of states other than in expressions of the form $\operatorname{Holds}(f, z)$, and without actions or situations. If $\Delta(z)$ is a state formula and $s$ a situation variable, then $\Delta(z)\{z /$ State $(s)\}$ is a situation formula, written $\Delta(s)$." [Thielscher, 2005, p. 14]

The agent program has to know, which action or sequence of actions is executable in order to achieve optimal, goal-directed behaviour and to avoid the risk of causing harm to itself or its environment. So-called precondition axioms define precisely, whether the given action is executable in a certain situation. In general, a precondition axiom is a formula $\operatorname{Poss}(A(\vec{x}), z) \equiv \Pi(z)$, whereof $\Pi(z)$ is a state formula which determines the predicate Poss, which is defined as: 
The agent's internal model of the world has to be maintained by the agent program itself, of course. For this purpose, so-called state update axioms specify the changes to the world model caused by the agent's actions. For each (kind of) action of the cognitive agent, the axiomatization of the application domain includes one such state update axiom. This axiom determines the subsequent state $\operatorname{State}(\operatorname{Do}(A(\vec{x}), s))$ given the state State $(s)$ after executing a certain action $A(\vec{x})$ in situation $s$ as follows:

$$
\begin{aligned}
& \operatorname{Poss}(A(\vec{x}), s) \supset \\
& \left(\exists \vec{y}_{1}\right)\left(\Delta_{1}(s) \wedge \operatorname{State}(\operatorname{Do}(A(\vec{x}), s))=\operatorname{State}(s)-\vartheta_{1}^{-}+\vartheta_{1}^{+}\right) \vee \cdots \vee \\
& \left(\exists \vec{y}_{n}\right)\left(\Delta_{n}(s) \wedge \operatorname{State}(\operatorname{Do}(A(\vec{x}), s))=\operatorname{State}(s)-\vartheta_{n}^{-}+\vartheta_{n}^{+}\right)
\end{aligned}
$$

where $n \geq 1$ and where the $\Delta_{i}(s)$ with $i \geq 0$ are situation formulas with free variables among $\vec{x}, \vec{y}_{i}$ and $s$. The state update axioms utilize fluent removal of states $\vartheta_{i}^{-}$and fluent addition of states $\vartheta_{i}^{+}$, which may involve variables among $\vec{x}, \vec{y}_{i}$. The states $\vartheta_{i}^{-}$and $\vartheta_{i}^{+}$are called negative and positive effects of the considered action respectively. The terms $\Delta_{i}(s)$ specify conditions under which case $i$ applies. In this vein, state update axioms allow to model conditional and non-deterministic effects of actions.

Non-deterministic actions and incomplete knowledge about the dynamic world surrounding the cognitive agent give rise to the existence of a multitude of possible states that the world might be in according to the current, limited information of the agent, in general. For this purpose, the predicate $\operatorname{KState}(s, z)$ represents a so-called knowledge state, whereof an instance means that state $z$ is a possible subjective state of the world in situation $s$ with KState $:$ SIT $\times$ STATE. More formally, a knowledge state is a formula $\operatorname{KState}(s, z) \equiv \Phi(z)$ with $\Phi(z)$ being a state formula. This concept is one of the most useful features of the Fluent Calculus with respect to our symbol anchoring approach, because it allows to represent and to maintain multiple possible hypotheses of incomplete knowledge about the world in parallel. Alternative states may be associated with the same situation, if the state formula $\Phi(z)$ is a disjunction of states.

Example 2.1 A simple knowledge state of a certain situation $S$, that includes the two states, $z_{1}$ and $z_{2}$, and possibly further fluents that are shared by both $z_{1}$ and $z_{2}$, is represented easily as:

$$
(\exists)\left(\operatorname{KState}(S, z) \supset\left(z=z_{1} \circ z^{\prime} \vee z=z_{2} \circ z^{\prime}\right)\right)
$$

Given several possible world models, it is critical to define what it means for an agent to actually know something. In the Fluent Calculus the agent is said to know a property in a certain situation, if it holds in all possible states of that situation. Even complex expressions may be evaluated, which are called knowledge expressions. A well-formed knowledge expression can be composed of fluents, predicates Poss $(a)$ of action $a$, or other atoms $P(\vec{t})$ excluding situation and state terms, which are linked using standard logical connectives. Given a query $\phi$ in form of a knowledge expression, the Fluent Calculus defines how to evaluate whether the agent knows $\phi$ in situation $s$ like this:

$$
\operatorname{Knows}(\phi, s) \stackrel{\text { def }}{=}(\forall z)(\operatorname{KState}(s, z) \supset \operatorname{HOLDS}(\phi, z))
$$

The macro $\operatorname{HOLDS}(\phi, z)$ replaces each fluent $f$ in the knowledge expression $\phi$ with $\operatorname{Holds}(f, z)$ and each predicate $\operatorname{Poss}(a)$ with $\operatorname{Poss}(a, z)$. The above definition of macro Knows implements that a given knowledge expression holds in a certain situation, if this expression holds in all possible states associated with the given situation.

We call $\Sigma_{F C}$ the foundational axioms of Fluent Calculus that include the set $\Sigma_{\text {state }}$ of axioms and the following axiom for knowledge:

$$
(\forall s)(\operatorname{KState}(s, \text { State }(s)))
$$

A knowledge state has to be updated upon perception of new sensory data or upon the execution of actions by the agent. Basically, the same principle as for the above-mentioned state update 
is used for the update of a knowledge state. A so-called knowledge update axiom determines the knowledge in the successor situation $D o(A, s)$ of $s$ right after performing action $A$ in $s$ :

$$
\begin{aligned}
& \operatorname{Poss}(A(\vec{x}), s) \supset \\
& \quad(\exists \vec{y})\left(\forall z^{\prime}\right)\left(\operatorname{KState}\left(\operatorname{Do}(A(\vec{x}), s), z^{\prime}\right) \equiv\right. \\
& \left.\quad(\exists z)\left(\operatorname{KState}(s, z) \wedge \Psi\left(z^{\prime}, z\right)\right) \wedge \Pi\left(z^{\prime}, \operatorname{Do}(A(\vec{x}), s)\right)\right)
\end{aligned}
$$

Hereof, the formula $\Psi\left(z^{\prime}, z\right)$ denotes the physical effects of action $A(\vec{x})$ similar to the state update axiom described above. The cognitive effect formula $\Pi\left(z^{\prime}, D o(A(\vec{x}), s)\right)$ specifies that all possible successor states $z^{\prime}$ have to agree with the sensory information available in the situation after executing action $A(\vec{x})$.

The reader is referred to the exhaustive explication of the Fluent Calculus in [Thielscher, 2005] for further details. 


\section{Chapter 3}

\section{Our Approach to Symbol Anchoring}

This chapter describes the general ideas and concepts behind our approach to the symbol anchoring problem. It also includes the assumptions posed by this approach about certain functionalities of other modules of the control architecture of the cognitive robot. The general concepts and the assumptions in this chapter apply to both the uni-modal version and the multi-modal version of our symbol anchoring approach, presented in Chapters 4 and 5 respectively.

The general aim of our work is to develop a well-founded approach to the symbol anchoring problem, which is applicable to modern, cognitive robots equipped with sensors and actuators for perception and interaction, which are situated in the real world. Hence, the platform addressed in our approach is an embodied, cognitive, autonomous robot, which is typically but not necessarily mobile. Thus, embodiment of the agent being a requirement of the symbol grounding problem is fulfilled by assumption (cf. Section 2.2.3).

While our work emphasizes a formal specification, this approach addresses practical, cognitive robots and is not limited to certain application domains. In fact, we carefully designed this approach to be completely independent of the application domain and of the robot platform. We believe that any action planning strategy that analyses the symbol anchoring hypothesis space and decides how to proceed appropriately, involuntarily involves domain-specific heuristics. Therefore, our approach does not incorporate action planning. For example, while it makes perfect sense for a service robot in a tidy home environment to put all its sensorimotor capacity to good use and to wander around looking for the object in question autonomously, this degree of autonomy in action selection in terms of symbol anchoring would be very costly, inefficient and even risky for a planet rover on a hazardous, extraterrestrial planet. However, in the Section 6.2 on future work we provide some guidelines and summarize which criteria should be taken into account, when our symbol anchoring approach is to be integrated into an action planning strategy.

Our suggested approach takes incomplete perception and incomplete observability of the robot's environment, as well as perceptual uncertainty into account in order to comply with real-world conditions. Thus, the internal model of the objects in the world necessarily becomes incomplete and uncertain, which is also called the common-sense informatic situation [McCarthy, 1989]. Therefore, our approach especially facilitates multi-modal perception, i. e., the capacity of the robot to employ several different sensors and to appropriately interpret the perceptual information, because the availability of rich perceptual information allows the robot to synchronize its internal world model with the real world in detail. Furthermore, multi-modal perceptual information about the same object of the world obtained from multiple, different sensors typically facilitates to disambiguate a situation and helps to cope with a complex environment including various kinds of objects.

Our approach is based on first-order logic with sets and equality, and on the Fluent Calculus as described in Section 2.4. The choice for this particular language was due to our requirements with respect to functionalities and expressiveness, including e.g., to represent abstract knowledge concisely; to reason about objects, their properties and relations between objects of the world; to represent uncertainty and incompleteness of information; and to represent and to reason about multiple alternative world models, which are to be maintained simultaneously and efficiently. 
During the study of the symbol anchoring problem we have identified several fundamental ideas to be crucial for a symbol anchoring approach in general. Therefore, the conception of and argument in favour of the approach presented below are based on these ideas. Some of these ideas will be proved to hold in this approach mathematically below, while others demonstrate rational principles of symbol anchoring.

\subsection{Independence of Information}

Those control architectures of cognitive robots, which the symbol anchoring problem applies to, are typically made of a symbolic, deliberative level for knowledge representation and logical reasoning on the one hand, and a sensorimotor level for processing of sensor data and for controlling the robot's effectors on the other hand (cf. Section 2.3.1). ${ }^{1}$ This distinction with respect to processes and representations is illustrated in Figure 2.1 on page 13 in a vertical composition. An important observation is that the source of the information about objects in the world at each level is very different. The sensory data of physical objects processed by the sensorimotor level originate from the robot's sensors. In contrast, symbolic information about objects in the world on the symbolic level is known a priori, learned, derived or was gained from communicating with other agents or humans. The first fundamental principle of our symbol anchoring approach for cognitive systems is that the information processed individually on the deliberative and sensorimotor levels must be kept separate, i.e., the deliberative information shall remain completely independent of the sensorimotor information and vice versa. This principle is justified by the same reason which gives rise to the symbol anchoring problem itself: The correct correspondence between an object identifier on the symbolic level and sensory data of a physical object is not "given" in the sense of philosophy of cognition (cf. Section 2.1.2); i.e., the cognitive robot is neither directly aware of the correspondence, nor can the robot be absolutely certain about the correctness of a correspondence. A correspondence between an object identifier and sensory information about an object in the world should be considered as a belief in a link between the two entities, which is possibly sustained by information on both levels individually.

Let's consider an example illustrating the effects of not following the described principle of independence of symbolic and sensorimotor information. Imagine a cognitive agent who's task is to perform some actions on a particular object in the environment. First, the agent has to find the object, i. e., it has to search the environment for such kind of object of interest, and then has to determine the one candidate which matches the given specification of the desired object best. Once the agent has found an appropriate object with characteristics that justify the agent to believe that it has found the right object, the agent may start to act on this object. As soon as the perception of or interaction with the object causes a contradiction between the specification of the object that the agent has in mind and the physical object's perceived characteristics, the agent's belief in the object being the right one has to be revoked. Thus, the correspondence between the object identifier of the object the agent had in mind and the percept obtained from an object in the world was incorrect - the object identifier should be anchored to a different, appropriate percept instead, and the current percept has to be associated with a different identifier. In this situation, it is crucial for the agent to be able to revoke the correspondence and the information about the two entities accurately. However, if the sensorimotor and symbolic processes have derived information from each other such that an intertwined world model resulted, then the revocation of the mingled information about the two different objects - the one denoted by the object identifier, and the one perceived - cannot be achieved accurately or may even be impossible. In contrast, a control architecture which complies with our concept of independent symbolic and sensorimotor information would easily be able to accurately revoke a mistaken correspondence linking the two levels by means of symbol anchoring such that the mistake has not affected the world model at all.

\footnotetext{
${ }^{1}$ The term "level" is used in this context to denote a complete, functional module composed of processes and representations.
} 
In comparison with related approaches, the symbol anchoring approach of Chella et al. [2004] incorporates perceptual as well as abstract symbolic information into the same representation in order to allow for a direct comparison between a symbolic description of an object and its measurements (cf. Section 2.3.5.3). This approach violates our statement about independence of the two levels clearly, because the algorithms for updating anchor representations fuse symbolic and perceptual information heuristically, for example.

The original, basic approach of Coradeschi and Saffiotti [2001] seems to keep symbolic and perceptual information separate. However, not all details are disclosed including the implementation of the function to predict the perceptual signature of an object. This function incorporates symbolic as well as perceptual information and thus may violate our principle of independence of information.

The Fuzzy logic approach of Coradeschi et al. [2001] appears to conform to our principle of independence proposed above. Yet their approach does not link to an expressive symbolic reasoning system at all, it just uses a couple of linguistic variables as symbolic predicates.

\subsection{Philosophy of Cognition}

Adopting the philosophy of cognition for cognitive robots, our approach follows the sense-data theory, because the robot's perception is implemented using processes and representations which provide indirect interpretations of the sensory data to other processes (cf. Section 2.1.2). Due to the limited immediacy of perception, a cognitive robot may also experience so-called perceptual seemings (e.g. illusions or hallucinations). We disregard these phenomena in our approach in order to avoid the considerable complexity this would entail otherwise. For instance, in case an illusion caused an inconsistency in the example above, which in turn necessitated to revoke the correspondence at hand, imagine the illusion was uncovered later such that the revocation of the correspondence has to be reversed.

Surprisingly, the human brain seems to have the capability to easily revoke wrong beliefs and to switch to an alternative hypothesis, which then is available immediately or can be constructed quickly. As we consider this functionality to be highly desirable for a cognitive robot to exhibit intelligent behaviour, our approach is designed to model this capacity of maintaining multiple hypotheses and to switch between alternative ones easily, while maintaining consistent, individual hypothesis representations. In our explanations below we will point out that our approach complies with further concepts of philosophy of cognition, too, e. g. representationalism and phenomenalism.

\subsection{Object Categories}

In general, a cognitive robot knows various kinds of objects of its environment, which it has to be able to recognize and to interact with. Typical, single actions of a robot focus on either an individual object or on a certain kind of object. Therefore, it appears not necessary for the object recognition system to notice all kinds of objects at once that it is able to recognize at all, but to focus its attention on the kind of object in question in the current task in order to solve it efficiently. Therefore, our approach uses object categories for symbol anchoring and for object recognition, such that both processes are limited to objects of the same kind. For this we assume a static association of objects with a category expressing the object's typical characteristics; in other words, an object is assumed not to change its category over time. All objects of the same category share similar perceptual characteristics. Hence, the robot's sensing actions should be designed for recognizing all objects of the given object category within the perceptual ranges of the sensors such as in model-based object recognition, because each perceived object represents a potential candidate according to its perceptual characteristics for symbol anchoring of the correspondence in question. The main idea for using object categories is to take all potential candidates that have been perceived with expected characteristics into account for symbol anchoring, because the aim of symbol anchoring is to determine the right object or the most appropriate object among all 
candidate percepts. Nevertheless, this concept should not restrict the application domain.

The object models used for recognition specify those phenomenal characteristics that are crucial and typical for recognizing objects of the considered category (cf. Section 2.1.2). While the object models serve to distinguish between objects of different categories, symbol anchoring especially facilitates the identification of individual objects of the environment. For this purpose symbol anchoring takes a symbolic specification of the object in question in terms of phenomenal characteristics. Note that the meaning of many linguistic terms that are used in natural language to denote phenomenal features of objects depend on the context. For example, the term "big" apparently bears a different meaning concerning the category "house" than concerning the category "dog". Our approach takes the dependence on context into account and utilizes object categories as a sufficient description of the context of phenomenal, linguistic terms. This is known as "pragmatic interpretation" in natural language processing and is part of the process of human communication [Russell and Norvig, 2003]. Formally, we introduce a separate sort OBJCAT made up of all categories of objects which the cognitive robot is required to know for the intended task. See also Section 3.6 for further advantages of object categories in this symbol anchoring approach.

If a very modular and flexible approach to the representation and reasoning about object properties and categories is in question, then ontologies of objects could provide some benefits. An ontology typically maintains a concise and uniform representation of properties and categories of objects, which facilitates efficient reasoning. Therein, multiple, more special categories can inherit common properties from parental nodes of the ontology tree (or network), for instance. Our approach does not employ an ontology, as we focus on symbol anchoring.

The Fuzzy logic approach of Coradeschi et al. [2001] seems to take object categories into account implicitly using specific definitions of perceptual predicates for each category, e. g. "smallsuitcase" (cf. Section 2.3.5.2). Apparently, only the early paper of Coradeschi and Saffiotti [1999] employs object categories explicitly for focusing object recognition and symbol anchoring. Especially the basic approach of Saffiotti's group described in Section 2.3.5.1, which several improved versions are based on, no longer makes use of object categories [Coradeschi and Saffiotti, 2001].

\subsection{Each Percept-One Symbol}

In Section 3.3 above, we have already argued that each potential candidate percept has to be taken into account for symbol anchoring in order not to miss the right object. The model-based object recognition looks for objects in the current perceptual range that exhibit the phenomenal characteristics which are typical of objects of the given category, such that each measured percept represents a potential candidate for symbol anchoring. Therefore, symbol anchoring must not neglect any of these percepts. It is crucial to make sure that each percept gets associated with an object identifier during symbol anchoring in order to provide a handle for each potential candidate percept, i. e. an anchor (cf. Section 2.3.2). Note that this statement tells us nothing about which particular symbol is to be chosen for a correspondence with a certain percept, but rather that some identifier has to be associated with a percept. It is left to symbol anchoring and logical reasoning to determine which one among the possible object identifiers is the right one for a given percept.

The suggested concept does not assume perfect perception or object recognition in the sense that if there is a physical object present within the range of some sensor with characteristics meeting the specification, then the object recognition system is assumed to notice it. In contrast, imperfect object recognition is taken into account in our approach, such that there is no guarantee that the desired object of the environment can be actually perceived.

Each individual object which the cognitive agent knows about or experiences is denoted by a unique identifier on the symbolic level in our approach. We distinguish between two distinct sets of object identifiers. The set $\mathcal{X}$ comprises the identifiers of all those objects that are known by the agent initially. All other objects, which the agent experiences from percepts later during its lifetime, will be assigned identifiers of the countably infinite sort PERCID. For this purpose we simply take the natural numbers, PERCID $=\mathbb{N}$. 
In contrast to our approach being based on the above-mentioned concept, the symbol anchoring approach of Chella et al. [2004] does not necessarily associate a symbol with each new percept (cf. Section 2.3.5.3), for example. Hence, their approach may leave out the correct correspondence. With the basic symbol anchoring approach of Coradeschi and Saffiotti [2001] a cognitive robot cannot learn about completely new objects in its environment because it is limited to unidirectional symbol anchoring: Given an object identifier, their functionalities are designed to anchor suitable percepts in a top-down manner (cf. Section 2.3.5.1). In contrast, our approach anchors percepts to symbols and vice versa bidirectionally, as you will see below in Chapters 4 and 5. Hence, the approach of [Coradeschi and Saffiotti, 2001] cannot guarantee to assign an object symbol to every percept.

The approach of Chella et al. [2004] summarized in Section 2.3.5.3 demonstrates in functionality "Find" that percepts may not be associated with an object identifier. The Fuzzy logic approach of [Coradeschi and Saffiotti, 1999] essentially lacks a symbolic level including object identifiers altogether.

\subsection{Uncertainty Representation}

Imprecision and noise due to real-world perception are among the practical challenges of symbol anchoring approaches for cognitive robots (cf. Section 2.3.3.1). Probability distributions are an expressive means for representing uncertainty and are quite popular in cognitive robotics [Thrun et al., 2005]. Our approach takes a trade-off between efficiency of computation and expressiveness of the representation. The representation chosen here is a one-dimensional interval $(\mathbb{R} \times \mathbb{R})$ of continuous, perceptual values for each perceptual attribute with the meaning that any value within the given bounds is valid due to the uncertainty, noise and imprecision of perception. In other words, the true perceptual value of the object's measured attribute actually lies somewhere within the perceived interval of perceptual values.

For a comparison with the Fuzzy logic theory, the Figures 3.1 and 3.2 depict examples of a Fuzzy number, Fuzzy intervals and Fuzzy operations, as well as the relation between their respective representation in Fuzzy logic and the corresponding representation of our approach in form of one-dimensional intervals. Given the membership function $\mu: X \mapsto[0,1]$ for elements $x$ of the finite domain $X$, we define the corresponding one-dimensional interval to contain those elements $x \in X$ where $\mu(x)>0$. Note that the bounds of the Fuzzy number of Figure 3.1(a) are not contained in the corresponding one-dimensional interval, because $\mu\left(x_{1}\right)=\mu\left(x_{2}\right)=0$. Similarly, the bounds $x_{1}$ and $x_{4}$ of the Fuzzy interval of Figure 3.1(b) are not contained in the corresponding one-dimensional interval either. Figure 3.1(c) illustrates the Fuzzy negation operator applied to Fuzzy interval $E$ depicted in Figure 3.1(b).

The Fuzzy logic operator AND can be considered the intersection of the two input Fuzzy intervals as exemplified in Figure 3.2(a). In terms of the one-dimensional representation chosen for our approach, the resulting interval depicted at the bottom of Figure 3.2(a) closely resembles the intersection of the two input intervals, but leaves out the bounds. Conversely, the Fuzzy logic operator OR computes the union of the two input intervals as illustrated in Figures 3.2(b). The Fuzzy OR of two distinct Fuzzy intervals yields a combination of the two input intervals. The bottom of Figure 3.2(c) depicts how the one-dimensional representation used in our approach works with multiple input intervals. Applying the Fuzzy logic operator AND to two distinct Fuzzy intervals results in a Fuzzy set with $\mu(x)=0$ for all $x \in X$ yielding a uniform possibility distribution. Our approach represents this special case in form of the empty interval $(x ; x)$ for some $x \in X$.

In the above, one-dimensional way, the interval representation of perceptual uncertainty is concise enough for reasoning in first-order logic, but still provides sufficient means to maintain individual degrees of sensory imprecision and noise. In comparison with Fuzzy logic operations and the Fuzzy interval representation, the one-dimensional intervals employed in our approach seem to lack varying degrees of certainty only. But this might not be crucial in a cognitive robotics system equipped with various sensors of different perceptual precision which are affected individually by 


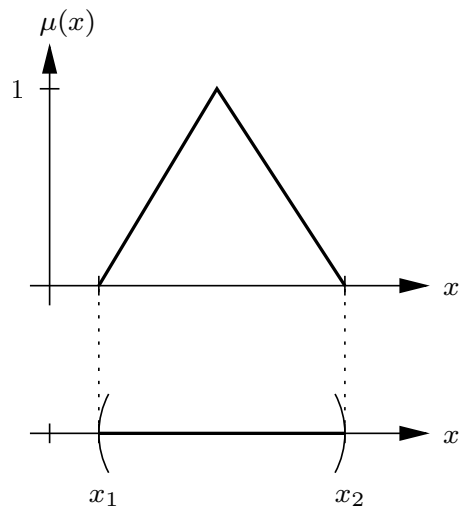

(a) A Fuzzy number.

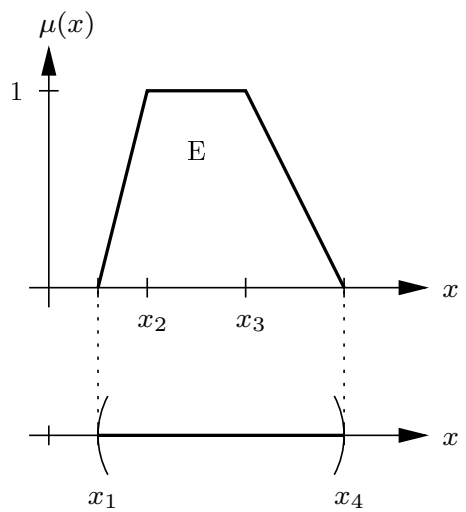

(b) A Fuzzy interval.

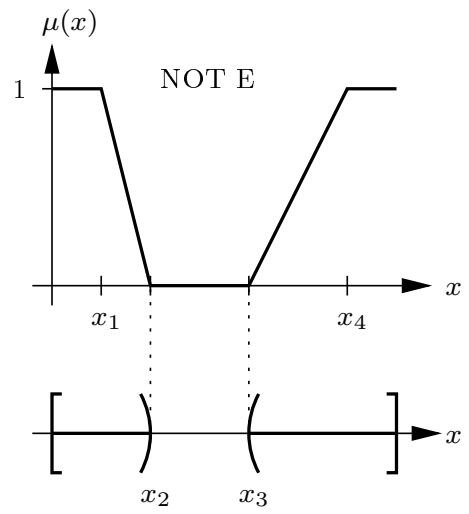

(c) Fuzzy NOT of the interval (b).

Figure 3.1: Top row: Basic Fuzzy entities and the Fuzzy NOT operation. Below each diagram is the corresponding one-dimensional interval.
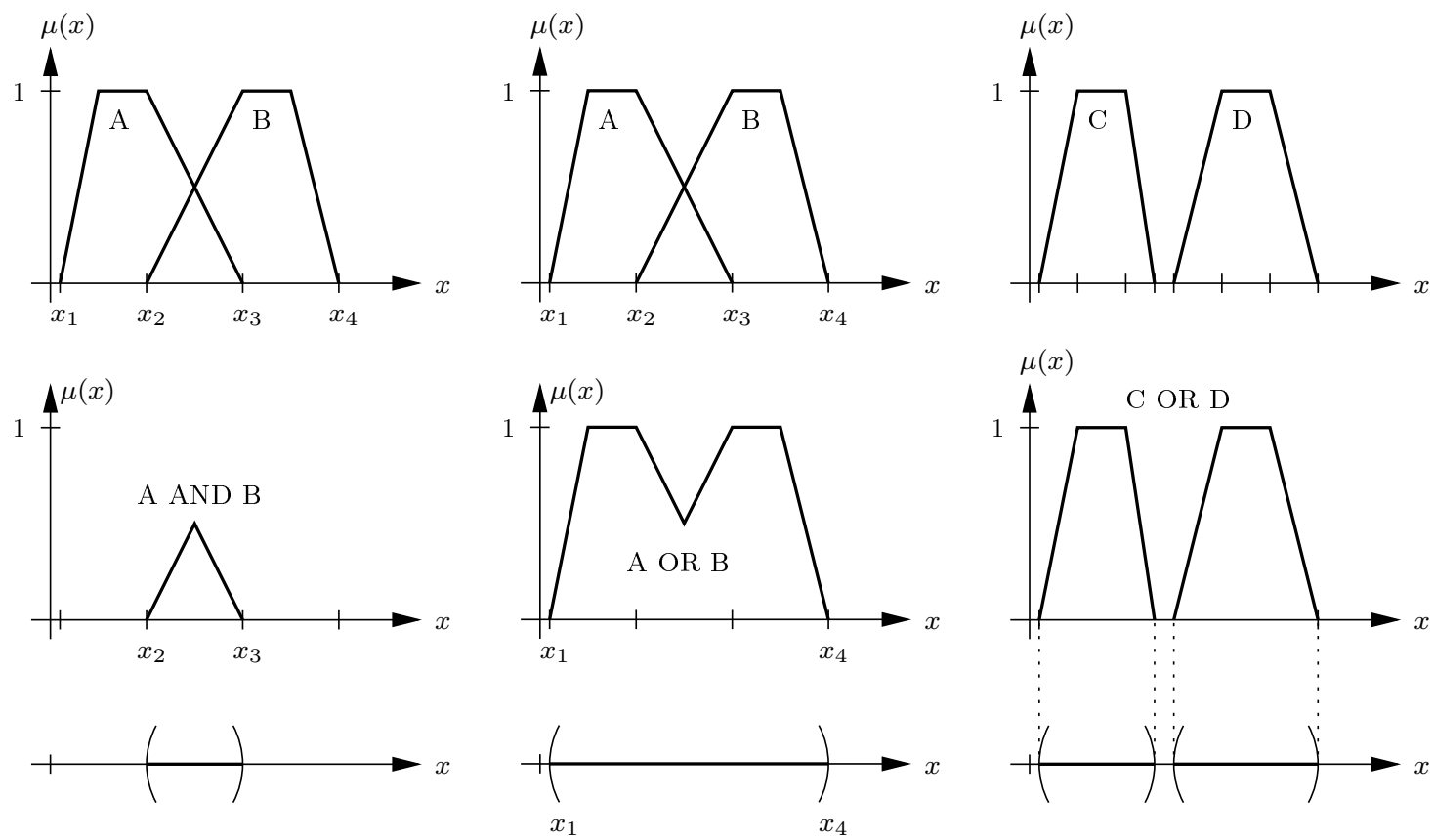

(a) Fuzzy AND of two overlapping

(b) Fuzzy OR of two overlapping Fuzzy intervals.

(c) Fuzzy OR of two separate Fuzzy intervals.

Figure 3.2: The top row illustrates three different pairs of input Fuzzy intervals. Below each of them, the result of applying the Fuzzy logic operations AND or OR is given in terms of Fuzzy intervals (middle row) and one-dimensional intervals (bottom row) respectively. 
different degrees of noise. Furthermore, typical perception systems of cognitive robots combine various sources of uncertainty into a single measure, e.g. imprecision, incompleteness, noise, unreliability, erroneous data, inaccuracy and others. But the accuracy of the fusion process seems questionable.

The above method suits our approach well: We neglect various degrees of certainty, but utilize the bounds of perceptual intervals in reasoning about these constraints. Hence, any uncertainty involved in perceiving the requested attribute is to be included in the resulting interval of perceptual values. Given this meaning of the interval representation, a reasoning system can utilize the perceptual information as a kind of constraint, because the actual perceptual values must lay inside the measured bounds and do definitely not fall outside the interval. Apparently, this kind of uncertainty representation requires the objective perception for our approach described in Section 2.3.3.4, i. e., the preprocessing of raw percepts should prevent environmental conditions to distort intervals of perceptual values. The interface between our symbol anchoring approach and the object recognition systems will be defined in detail below.

The Fuzzy logic approach of Coradeschi et al. [2001] suggests to represent perceptual uncertainty by means of possibility distributions in their theoretical, computational framework, but actually uses plain, continuous intervals - equally to our approach (cf. Section 2.3.5.2). The basic symbol anchoring approach of the group of Saffiotti neglects perceptual uncertainty completely, since the perceptual signature is defined as a partial function, $\gamma: \Phi \mapsto D$, which maps attributes to the domain $D$ of perceptual values (cf. Section 2.3.5.1). Similarly, the approach of Chella et al. [2004] summarized in Section 2.3.5.3 neglects perceptual uncertainty altogether, because an individual percept is mapped to a single point in the multi-dimensional conceptual space, i. e., the representation as well as the algorithms presented are restricted to individual values of perceptual attributes.

\subsection{Grounding of Predicates}

According to the symbol grounding problem, the symbols of the symbol system inside an agent have to be intrinsic and meaningful to the agent itself (cf. Section 2.2.2). The intrinsic meaning of the agent's symbols may only arise from causal connections with the environment, which should to be realized through rich interaction, e.g. recognition of phenomenal features of objects of the environment, acting on objects by means of the agent's effectors, and perceiving the environment using sensory transducers.

A symbolic description is used in symbol anchoring to refer to objects in the environment that exhibit the given phenomenal characteristics, as well as for reasoning about objects and identifying them. The specification includes predicates denoting perceptual attributes and their values. For example, the cognitive agent might know the perceptual attributes "colour", "height" and "odour", as well as the corresponding values "red", "tall" and "vanilla". Obviously, the meaning of these terms taken alone is parasitic on the human interpretation [Roy, 2004, Anderson, 2003a]. For this purpose, our approach introduces a relation between perceptual predicates and intervals of perceptual values, which realizes the grounding of abstract predicate symbols in sensorimotor terms. Our approach simply presupposes these relations to be pre-defined appropriately by the human programmer, who axiomatizes the robot's application domain.

These grounding relations are used in a static way while the application is running, because the reasoning involved in symbol anchoring depends on them. Adapting the grounding relations dynamically would cause undesirable effects if used in our approach. Inconsistencies would arise after changing a grounding relation, because the same description of an object would correspond to a different set of percepts suddenly, and correspondences of previous descriptions affected by the change would become invalid after the change automatically.

The learning of the grounding relations seems to be a very appealing idea as has been fostered by a number of researchers, e. g. Harnad [2005] and Davidsson [1993]. However, evolutionary algorithms, learning from examples and learning by observation have been stated to break the so-called zero semantical commitment condition, and therefore argued to fail to solve the symbol grounding 
problem according to Taddeo and Floridi [2005] (cf. Section 2.2.4.3). As symbol grounding is not the focus of our work, the above-mentioned static relations between predicates and sensorimotor information provide a reasonable solution to symbol grounding in our approach.

Example terms of perceptual values of attributes, e.g. "tall", "light", and "strong", illustrate their significant degree of dependence of their meaning on the context in which such terms are used. Extending the idea to focus object recognition and candidate percept selection on individual object categories as described in Section 3.3, our approach utilizes the same object categories in order to account for the context of perceptual predicates. In other words, the meaning of a perceptual attribute should be sufficiently clear given the object category under consideration. Reconsidering our previous examples, we seem to have a clear understanding of these descriptions if the object category is added: a tall building, a light weighed video camera or a strong odour of vanilla. We represent such relations in form of the predicate

$$
\text { Grounded : OBJCAT } \times \text { ATTR } \times \text { PERCPRED } \times(\mathbb{R} \times \mathbb{R})
$$

where all known object categories constitute the sort OBJCAT, where ATTR is the sort which holds all perceptual attributes and where PERCPRED is the sort containing all perceptual predicates denoting attribute values. The last constituent of predicate Grounded represents the interval of continuous, perceptual values as a tuple of real numbers, which the perceptual predicate is associated with. For example, the application domain may include cups of regular size, which could be represented as Grounded(Cup, Height, Regular, $(8,11)$ ) meaning that cups of regular size typically have a height between 8 to $11 \mathrm{~cm}$.

Values of perceptual attributes like "height" or "weight" may or may not change, depending on the individual attribute and on the kind of object. Since this dependence influences the reasoning of our symbol grounding approach, a definition of the kind of attribute is required for each attribute using the predicate

$$
\text { AttrTypeStatic : OBJCAT } \times \text { ATTR }
$$

This way, predicate AttrTypeStatic determines whether a given perceptual attribute is static depending on the object category under consideration. The predicate requires a consistent axiomatization of the application domain such that individual instances of this predicate and their negation are mutual exclusive. For example, the domain of a service robot may include cups with varying weight depending on how full they are, which is represented as $\neg$ AttrTypeStatic (Cup, Weight). ${ }^{2}$

In comparison, the Fuzzy logic approach of Coradeschi et al. [2001] is based on linguistic variables that are mapped to Fuzzy sets (cf. Section 2.3.5.2). Accordingly, this representation of vagueness provides improved expressiveness, but may also entail a higher a priori bias of the human programmer. The basic symbol anchoring approach in [Coradeschi and Saffiotti, 2001] of the same research group does not allow to take vagueness into account, since the grounding relation, $g \subseteq \mathcal{P} \times \Phi \times D$, directly refers to a single value of the domain $D$ (cf. Section 2.3.5.1). Recalling what we recognized with respect to categories of objects in Section 3.3, their approach does not take such categories into account explicitly. A predicate grounding relation that depends on the category of objects offers significantly better modularity and provides the means to infer characteristics of objects and to reason about them. The distinction between static and dynamic object attributes in our approach could be easily integrated in the approach of Coradeschi and Saffiotti [2001] into their function to predict the attribute values.

The approach of Chella et al. [2004] briefly summarized in Section 2.3.5.3 could be considered a generalization of our approach with respect to the grounding of the predicates' meaning, because their grounding function $g: \mathcal{P} \mapsto 2^{K}$ maps perceptual predicates to multi-dimensional sub-spaces of a conceptual space. However, most perceptual predicates, e.g. "height", "weight", "odour" or "hue" correspond to one-dimensional attributes only.

\footnotetext{
${ }^{2}$ See Section 2.3.3.5 for the reason why we do not distinguish between the content of the cup and the cup itself.
} 


\subsection{Symbolic Descriptions of Objects}

The symbolic description of an object in our work refers to the cognitive knowledge of an agent on the deliberative level about the object of the agent's environment (cf. Figure 2.1). Recall our concept of independence of perceptual and symbolic information, stating that information must not be exchanged between the perceptual and symbolic levels (cf. Section 3.1).

The information modelled on the symbolic level may include all phenomenal characteristics that objects of interest exhibit, i.e., their phenomenal attributes and relations between objects. Such information could also be obtained through communication with other agents or humans, provided all participants refer to the same object of the world when they talk about a particular one. Of course, a priori symbolic knowledge can be incorporated, too. Also, given the symbolic knowledge available to an agent in a certain situation and given a sound deductive inference mechanism, the agent may infer additional explicit knowledge and represent it at the symbolic level. The Fluent Calculus - the first-order logic language used in our approach-empowers symbol anchoring agents based on our approach to draw such logical inferences (cf. Section 2.4).

Knowledge on characteristics of an object can also be obtained during interaction of the agent with the object, e.g. its weight, whether it is movable or breakable etc. Such features of objects are functional by nature. It is important to note that these characteristics result from the percept of an object, i. e., the sense data of a physical object. Hence, interactive features of objects must not be incorporated into the symbolic level, but have to remain at the perceptual level. Again, the reason behind this principle is the suggested mutual independence of symbolic and perceptual information as described in Section 3.1.

In general, a symbolic description can be used for indefinite as well as definite references to real-world objects (cf. Sections 2.1.1.2 and 3.8). In both situations the actual identifier of the objects is irrelevant to the task. In the case of an indefinite reference, we intend to find those objects in the agent's environment which match the given characteristics. As a result, none, a single or multiple references may be determined. A symbolic description for an indefinite reference does not point at a particular object identifier, because the use case of indefinite references is to find suitable objects. Therefore, if multiple matching references have been found, each one may meet the purpose equally well, because we were not looking for a particular object. Note that the information on the perceptual level is also involved-besides symbolic knowledge-in verifying the appropriateness of an object, if a correspondence between an object identifier and a percept is established.

Conversely, a definite description refers to a particular object in the agent's "mind". The ability to resolve the reference uniquely is a prerequisite to act on the intended object in the sequel. Hence, the number of matching objects actually matters. If no object matches, then the agent has simply not perceived or experienced the right object yet due to several possible reasons. If one object matches, planning and acting can proceed with the next step of the strategy. ${ }^{3}$ However, if multiple objects match the symbolic description, the description bears insufficient detail, is incomplete or the symbolic and perceptual levels contain insufficient characteristics in order to uniquely resolve the reference. A high-level action planning system for cognitive agents has to take these cases into account and to react appropriately deploying different strategies depending on the situation. Such a planning system is not part of our work, because it involves domain-dependent strategies in contrast to our domain-independent symbol-anchoring approach. Obviously, our approach lays the foundation for a specific action planning system.

Returning to our symbol-anchoring approach, in order to represent phenomenal properties of objects on the symbolic level, we introduce the new fluent Prop with the following definition:

$$
\text { Prop }: \mathcal{X} \cup \text { PERCID } \times \text { ATTR } \times \text { PERCPRED } \mapsto \text { FLUENT }
$$

\footnotetext{
${ }^{3}$ Only the fact that a definite reference given a symbolic description has been resolved doesn't mean that this object is precisely the assumed one. The system engineer for the particular application of the cognitive agent has to make sure that the appropriateness of an object is verified in terms of all relevant characteristics. Then, the object can be said to serve the purpose and be considered the right one according to the experience so far. The ground truth of whether the correct correspondence to a real-world object has been determined is basically not available to the cognitive agent by virtue of the exercise.
} 
The meaning of fluent $\operatorname{Prop}(x, a, p)$ is that the agent knows the value $p$ of the given perceptual attribute $a$ of a particular object $x$, if the fluent is grounded and holds in the state of interest. The first component of fluent Prop denotes the object uniquely by means of a constant identifier. The other two components represent the known attribute value of sort PERCPRED for the perceptual attribute of sort ATTR. The definition of fluent Prop above serves as a general, domain-independent interface for our approach to the symbolic knowledge of the agent on objects in its environment.

Apparently, the Definition (3.3) does not mention the object's category. It is unnecessary to name the object's category in the fluent Prop explicitly, because first, our approach maintains individual anchors defining the category of each object, and second, object identifiers are unique. This will be explicated in the following chapters.

Our approach offers a highly expressive means for specifying a symbolic description of an object: We employ a so-called state formula of the Fluent Calculus for representing the desired symbolic characteristics of an object using the fluent Prop (cf. Section 2.4). The state variable $z$ of a state formula $\Delta(z)$ refers to a certain symbol anchoring hypothesis. Using such a well-formed logical formula as a symbolic description affords several typical use cases:

1. The symbolic description may represent a definite reference in order to deduce information about a certain, given object.

2. Without specifying a particular state, the symbolic description may be used to reason about and to compare the knowledge of the given object in alternative symbol anchoring hypotheses.

3. The symbolic description may also be used as an indefinite reference to an object. Given the desired properties in form of the state formula, a suitable object can be inferred this way from the knowledge of a certain, given symbol anchoring hypothesis.

The symbolic description may also specify relational information or constraints between attribute values or among multiple, different objects. In general, our symbol anchoring approach should be considered as a layer providing the fundamental functionality of symbol anchoring, which allows full access to its knowledge representation in terms of symbol anchoring hypotheses.

In comparison with previous work, in the approach of Coradeschi and Saffiotti [2001] summarized in Section 2.3.5.1 the symbolic description of an object is implemented by means of a description state $D S_{t}: \mathcal{X} \mapsto 2^{\mathcal{P}}$. It associates the object symbol with a set of unary predicates. Apparently, this description state is limited to a conjunction of predicates only, while our approach may utilize the rich expressiveness of a logical formula for a symbolic description. The same applies to their other approaches and improved later versions, e.g. [Chella et al., 2004, Coradeschi and Saffiotti, 2000, 2002]. Coradeschi and Saffiotti [1999] do not describe how to implement a symbolic description for the Fuzzy logic version of their symbol anchoring approach. This approach lacks an integration with a symbolic reasoning system entirely.

\subsection{Reference to Objects}

The use of definite and indefinite references for symbol anchoring in general as well as in our approach is derived from the descriptive theory of philosophy of language regarding proper names (cf. Section 2.1.2). According to this theory, we may assume in our approach that a description in terms of properties resolves the referent to an object uniquely [Reimer, 2006]. For instance, Russell [1905] confirmed that the article "the" of English language indicates uniqueness of the given reference. His idea of using logic for analysing the semantics of natural language aimed at resolving the ambiguity and vagueness which are inherent in the language. In particular, the statement "The $x$ such that $P(x)$ " represents a definite description and is used to denote the particular object $x$. Conversely, the statement "An $x$ such that $P(x)$ " expresses an indefinite description and is intended to denote an arbitrary object of the class of P-objects [Russell, 1905, Coradeschi and Saffiotti, 2003b]. 
Our approach facilitates both definite and indefinite references of objects. Russel's above idea of representing definite and indefinite references of objects applies to our approach directly. The goals for the design of an action planning system for a cognitive agent would be first, to disambiguate the definite description and second, to anchor the object symbol to an appropriate real-world object. With regard to the first task, the possible means to verify whether an object symbol refers to the correct physical object of the agent's environment in our approach are as follows. First and foremost, the phenomenal characteristics of the object serve the purpose of recognizing and disambiguating the correspondence, but also the object's functional characteristics, features of interaction with it and relational attributes within the environment do so. Any such feature may sustain or disprove the correctness of a correspondence during the course of action. We strongly believe that an appropriate approach to the symbol anchoring problem has to take this dynamic aspect of a correspondence into account. Subsequently you will learn how our approach implements this functionality. Note that this approach complies with the phenomenalist's view of cognition (cf. Section 3.2).

For example, let's consider an indefinite reference to a light bulb with the symbolic description "an intact light bulb". Imagine that the cognitive robot recognized a light bulb in its environment. In order to verify the correctness of the reference, the robot has to fit the light bulb in a socket, turn on the currency and verify whether it lights or not. This is an example of functional characteristics of objects that require complex, domain-dependent action strategies, which is outside the scope of our approach. In general, disambiguation depends on the application domain and seems to be rather complex.

With regard to the second, above-mentioned task-to anchor the object symbol to an appropriate real-world object - the same rationale as above applies to what is to be considered an appropriate object: All known characteristics of an object shall be verified, depending on the sensory capacity of the cognitive robot. How the anchoring itself is performed in our approach precisely is the topic of the subsequent sections.

Comparing our approach with previous works summarized in Section 2.3.5, the Fuzzy logic approach of Coradeschi and Saffiotti [1999] essentially does not include concepts for symbolic references to objects. Given the limited expressiveness of symbolic descriptions of the basic symbol anchoring approach of Coradeschi and Saffiotti [2001], only simple definite references are available when a certain object symbol is to be anchored to an appropriate percept in a top-down manner. The same applies to the approach in [Chella et al., 2004], which is based on conceptual spaces (cf. Section 2.3.5.3). Saffiotti's group extended their approach to using Lisp terms for specifying definite and indefinite references to objects in more recent, improved versions [Broxvall et al., 2004a, 2005, Bouguerra et al., 2006]. These approaches lack sound, logically proven characteristics and are based on a rather procedural planner, in contrast to our approach.

\subsection{Symbol Anchoring Hypothesis}

Let's be more precise with respect to the meaning of a hypothesis. In general terms a symbol anchoring hypothesis, hypothesis for short in the sequel, is called a complete set of anchors, and symbolic and perceptual properties of objects, all of which belong to the same interpretation of a certain situation. The term "set of anchors" should not be taken literally. A hypothesis refers to a particular interpretation of the knowledge of the agent about all objects in a certain situation. A set of anchors is considered complete, if all anchors are included that are known in the situation and interpretation at hand. Anchors have to be unique within the same hypothesis for each object symbol, i. e., an admissible hypothesis does not contain more than one anchor with the same object symbol. Likewise, anchors have to be unique within the same hypothesis with respect to each percept identifier.

A symbol anchoring hypothesis is defined to enclose the symbolic and perceptual information about all known objects and their properties available in the situation considered. Here, we refer to all phenomenal characteristics represented on the symbolic and perceptual levels. Particularly, this part of the specification of a hypothesis includes a set of assignments of attributes to attribute 
values besides related constraints. All these properties have to be part of a hypothesis representation, which is the case if no further symbolic and perceptual properties of objects-both their associated symbols and percepts - are currently known which are consistent with the considered interpretation. Thus, an alternative symbolic or perceptual property that is also known to hold for a certain object, gives rise to a separate, alternative hypothesis because it refers to a separate interpretation of the same situation. In other words, a hypothesis is the smallest part of a certain state $z$ associated with the knowledge state by means of $\operatorname{KState}(s, z)$, which comprises all anchor fluents, all symbolic and perceptual properties, i.e., all instances of fluent Prop, and possibly formulas of relations and constraints on anchors and properties. The following example shall illustrate our concept of a symbol anchoring hypothesis.

Example 3.1 Consider a certain situation $S$ wherein the cognitive agent knows of two objects in its world denoted as $A: \mathcal{X}$ and $B: \mathcal{X}$, whereof both are cups. For the sake of simplicity let a single hypothesis exist in the given situation, wherein the agent recognized a correspondence of object $A$ with percept $\pi_{1}$ : PERCID and of object $B$ with percept $\pi_{2}$ : PERCID. Furthermore, the agent knows that object $A$ is of large size, the colour of object $A$ is not red, and object $B$ has red colour. Apart from that, the agent knows no further symbol-anchoring information on objects or properties of objects currently. Until we will introduce the proper representation of anchors used in our approach below, let's simply abbreviate the anchors of our example as the relations $\left(A, \pi_{1}\right)$ and $\left(B, \pi_{2}\right)$. The object categories, attributes and a priori identifiers involved in this example are:

$$
\begin{aligned}
\text { OBJCAT } & =\{\text { Cup }\} \\
\text { ATTR } & =\{\text { Colour }, \text { Height }\} \\
\mathcal{X} & =\{A, B\}
\end{aligned}
$$

The perceptual attributes Colour and Height are defined static in this example:

$$
\begin{gathered}
\text { AttrTypeStatic (Cup, Colour) } \\
\text { AttrTypeStatic (Cup, Height) }
\end{gathered}
$$

Let's also demonstrate how the symbolic attribute values are grounded on perceptual values:

$$
\begin{aligned}
& \text { Grounded (Cup, Colour, Red, }(340,375)) \\
& \text { Grounded (Cup, Height, Large },(11,20))
\end{aligned}
$$

Using the HSV colour space, we represent the perceptual values of colour in terms of hue values neglecting saturation and value dimensions, since they depend on environmental conditions. ${ }^{4}$ The HSV colour space facilitates to achieve a much more objective perception of the environment in contrast to other standard colour spaces, especially the RGB space (cf. Section 2.3.3.4). ${ }^{5}$ The hue dimension of colour is typically understood as a circular coordinate with values between 0 and some maximum value, e. g. 360 degrees. Therefore, the perceptual values represented in our example shall be interpreted using the mapping $f(x)=x \bmod 360$ in order to yield hue values of the colour of objects. Furthermore, cups with a height of 11 to $20 \mathrm{~cm}$ are considered large cups.

Neglecting the representation of the anchors of the objects $A$ and $B$ for a moment, which will be introduced in the next chapters, the above described situation is represented as follows:

$$
\begin{aligned}
& (\exists z)(\text { KState }(S, z) \wedge \\
& \quad \text { Holds }(\text { Prop }(A, \text { Height }, \text { Large }), z) \wedge \\
& \neg \text { Holds }(\text { Prop }(\text { A, Colour }, \text { Red }), z) \wedge \\
& \text { Holds }(\text { Prop }(B, \text { Colour }, \text { Red }), z))
\end{aligned}
$$

Note that the predicate KState provides the association between a certain situation and individual states (cf. Section 2.4). Now you might wonder what the difference between a so-called state of

\footnotetext{
${ }^{4} \mathrm{HSV}$ abbreviates hue, saturation, value.

${ }^{5}$ RGB abbreviates red, green, blue.
} 
the Fluent Calculus and a symbol anchoring hypothesis is. While the state comprises all available knowledge that the agent has of a certain interpretation of the situation considered, the symbol anchoring hypothesis only concerns information of the considered interpretation and situation which is relevant to symbol anchoring, i.e., only information about objects of the environment and their properties. Hence, only a subset of the knowledge represented in the corresponding state forms a hypothesis in general. Consequently, a hypothesis subsumes multiple states in general, because these states may include alternative pieces of knowledge which are irrelevant to symbol anchoring.

Example 3.1 (continued) Following up the previous Example 3.1, the Formula (3.4) can be rewritten as

$$
\begin{gathered}
\left(\exists z, z^{\prime}\right)\left(\text { KState }(S, z) \wedge z=\operatorname{Prop}(A, \text { Height }, \text { Large }) \circ \operatorname{Prop}(B, \text { Colour }, \text { Red }) \circ z^{\prime} \wedge\right. \\
\neg \operatorname{Holds}(\operatorname{Prop}(A, \text { Colour }, \text { Red }), z))
\end{gathered}
$$

which illustrates better how a symbol anchoring hypothesis abstracts from additional knowledge besides symbol anchoring and possibly additional states. More precisely, the state variable $z^{\prime}$ may contain additional knowledge in Formula (3.5), but the hypothesis abstracts from these additional pieces of information and subsumes all individual states that $z^{\prime}$ may give rise to, e.g. $z^{\prime}$ may include some further fluents $F_{i}$ with $i=1 \ldots 4$ :

$$
z^{\prime}=F_{1} \circ F_{2} \circ z_{1} \vee z^{\prime}=F_{3} \circ F_{4} \circ z_{2}
$$

where the fluents $F_{i}$ are irrelevant to symbol anchoring in Formula (3.6). In other words, we consider $z$ a single hypothesis, even though $z$ may comprise several substates. Note that $(3.4) \equiv(3.5)$ holds.

As our approach is fully integrated into the Fluent Calculus, it offers a rich, logical, declarative expressiveness especially regarding the specification of symbol anchoring hypotheses. A symbol anchoring hypothesis may also include constraints on anchor fluents or on properties of objects or on relations among them.

In contrast, the various versions of approaches of Saffiotti's group are procedural implementations based on a Lisp planning system (cf. Section 2.3.5) [Coradeschi and Saffiotti, 2000, 2001, 2002, Broxvall et al., 2004a]. More recently, they extended their approaches to take certain logical constraints on the objects' properties into account, although considering particular ones only [Broxvall et al., 2005, Bouguerra et al., 2006, Chella et al., 2004]. Furthermore in these recent approaches, an individual hypothesis of correspondences could be computed, although such a hypothesis was restricted to a conjunction of object properties and anchors only.

We will show below that our approach does not restrict the use of logical formulas for expressing and representing symbol anchoring hypotheses and constraints (see also Section 3.7). In contrast to the above-mentioned previous approaches, we were able to prove certain important characteristics of symbol anchoring to hold in our approach, as it is based on first order logic (see below).

\subsection{Multiple Hypotheses of Correspondences}

According to the above definition, a symbol anchoring hypothesis refers to a single interpretation of the situation which the modelled agent and the world are in with respect to symbol anchoring (cf. Section 3.9). Consequently, any alternative symbolic or perceptual property known of an object in the same situation, or any alternative possible reference of an object identifier to a different percept - or vice versa - gives rise to a separate, interpretation of the same situation. This way, the information of an alternative hypothesis in the whole is inconsistent with that of another hypothesis existing in the same situation. Otherwise, two hypotheses would coincide and logically collapse into a single hypothesis, just as the example above showed how a hypothesis may subsume multiple states (cf. Formula (3.6)). It is important to note that a symbol anchoring hypothesis only regards anchors, symbolic and perceptual properties and constraints on properties, but ignores the other knowledge available in the considered situation (cf. Section 3.9). 
The existence of alternative hypotheses may be due to several reasons:

- An incomplete symbolic description of an object may provide insufficient detail in order to distinguish between all perceived real-world objects that match the given description.

- Incomplete perceptual information or insufficient detail of perceptual measurements may disallow the agent to determine a unique correspondence with a symbolic description or with an object identifier given a definite reference. The inability to measure certain perceptual attributes due to limited sensory capacity of the agent or simply the fact that they have not yet been measured at all gives rise to ambiguous situations. Also, the perceptual resolution of the agent's sensing devices is limited in practice. Hence, the real world may expose multiple objects that are indistinguishable for the agent with respect to its sensory capacity. In all these cases multiple percepts may stand in as potential candidates for the same correspondence.

- Environmental conditions or undesirable circumstances of measuring the objects of interest may have caused a low perceptual precision only. For instance a disadvantageous perspective or movement of the agent or of the objects typically provokes perceptual noise, partial obstruction or limited exposure to the sensors in terms of time, distance etc. Similarly as above, this may prevent percepts to supply sufficient detail in order for the symbol anchoring system to determine the correct correspondence between the perceived object and the object symbol uniquely.

We believe that it is crucial for an approach to the symbol anchoring problem to maintain all potential hypotheses of correspondences in order not to miss the correct one. This is counted among the fundamental concepts of our approach, and will be respected in several parts of our approach below. Our approach is designed to take each alternative symbol anchoring hypothesis into account explicitly, as long as it constitutes a potential candidate hypothesis. A hypothesis is considered possible, if the knowledge available to the agent as well as the history of the hypothesis sustain this interpretation of the given situation consistently. Conversely, as soon as some evidence proves an inconsistency in the knowledge of the whole hypothesis, it has been proved to be invalid and must be be disregarded. A hypothesis may become inconsistent if perceptual information contradicts symbolic information concerning a certain object.

Example 3.1 (continued) Let's reconsider the above Example 3.1 with the agent's knowledge as represented in Formula (3.4). Accordingly, the agent knows explicitly that the colour of object $A$ is not "red". Assuming the domain constraint that objects bear a unique colour, and assuming that the next sensing action revealed that the colour of object $A$ actually is "red", the whole hypothesis turns out to be inconsistent. This example illustrates the rationale behind our symbol anchoring approach nicely: We have full confidence in the knowledge on the symbolic level, i. e., our approach does not implement degrees of truth or probabilities of statements. Since our approach excludes perceptual seemings (cf. Section 3.1), we may assume quite rightly that the perceptual information is true and refers to an object of the real world. The specification of the application domain of this example provided a further, very useful constraint on the attribute "colour". Given all this information we can deduce that the assumed correspondence between object symbol $A$ and percept $\pi_{1}$ is wrong. This turns the whole hypothesis inconsistent.

Among the most beneficial features of our approach is that the complete set of symbol anchoring hypotheses is maintained automatically. The approach takes care of generating new potential hypotheses, updating existing hypotheses with new information and recognizing as well as disregarding invalid hypotheses. These are the three major tasks of our symbol-anchoring approach. Each of them will be discussed below in detail. We call the full set of hypotheses, which are available in a certain situation, a hypothesis space.

In general, the concept of introducing an additional, alternative hypothesis in our approach is the following. Apparently first, some additional information with respect to the previous situation regarding symbol anchoring is required. The new information may suggest that an alternative, 
potential interpretation of the situation with respect to the current hypothesis space is necessary in order to explain the new evidence, if the new interpretation has not been covered by those hypotheses represented so far. In this case, our approach introduces an additional disjunctive state into the knowledge state of the considered situation. To this end the knowledge update axiom of our approach takes the corresponding hypothesis space of the previous situation, including the particular hypothesis which the new one is based on, and updates it given new information. As the Fluent Calculus provides a solution to the Frame Problem, the knowledge update of our approach being based on the Fluent Calculus only has to consider the necessary changes with respect to the representation of the original hypothesis (cf. Section 2.4).

Example 3.1 (continued) Let's illustrate the method of maintaining the hypothesis space with an example, reconsidering our above Example 3.1 with the hypothesis space of the situation $S$ as specified in Formula (3.4). Imagine, the agent has perceived a single object then: percept $\pi_{3}$ denoting a cup with a height of 12 to $13 \mathrm{~cm}$ and without any associative information with respect to the previous percepts $\pi_{1}$ or $\pi_{2} \cdot{ }^{6}$ Thus the correspondences $\left(A, \pi_{1}\right)$ and $\left(B, \pi_{2}\right)$ of the hypothesis of situation $S$ are lost in the successive situation $S^{\prime}$. However, the perceptual signature of percept $\pi_{3}$ matches the symbolic information the agent has on both objects $A$ and $B$. The resulting hypothesis space of the situation $S^{\prime}$ after performing the symbol-anchoring update of our approach then includes the following two hypotheses besides others:

1. Object $A$ corresponds to percept $\pi_{3}$, object $B$ has no corresponding percept; the symbolic information that $A$ 's height is large and its colour is not red, the colour of $B$ is red.

2. Object $B$ corresponds to percept $\pi_{3}$, object $A$ has no corresponding percept; the symbolic information that $A$ 's height is large and its colour is not red, the colour of $B$ is red.

Both hypotheses are based on the previous hypothesis taking over previous knowledge, because the new perceptual information conforms to the knowledge in situation $S$. The percept caused the previous hypothesis to branch and to introduce two new hypotheses as a replacement in situation $S^{\prime}$.

In case the new information of a sensor action sustains all hypotheses of the current situation, there is no need to discard a hypothesis or to introduce a new one. Instead, the knowledge update axiom revises each hypothesis and amends the perceptual information of individual objects upon new perceptual measurements. Each update of individual perceptual attributes must be consistent with the whole hypothesis for the sake of the sustenance of it.

In case an inconsistency with new perceptual information occurs, the knowledge update axiom of our approach invalidates each concerned hypothesis of the new situation automatically. Basically, the knowledge update defines that each hypothesis of the successive hypothesis space must be based on some previous hypothesis. It also requires that each successive hypothesis must conform to all available percepts. Furthermore, the axiom defines precisely, how the previous hypothesis has to be adapted given the new perceptual information. A successive hypothesis will only be created if the update is possible. In other words, in case of an inconsistency of perceptual information with a certain successive hypothesis, the knowledge update axiom simply disregards this successive hypothesis. If some previous hypothesis cannot be updated to some successive hypothesis due to inconsistencies, this previous hypothesis has to die out.

Example 3.1 (continued) Let's continue with the above Example 3.1 from situation $S^{\prime}$ wherein the agent knows of two hypotheses. Suppose the agent perceives the colour of the object which it recognized from the previous sensing action: The percept $\pi_{4}$ refers to the same physical object as before, i. e., $\pi_{4}$ refers to percept $\pi_{3}$, and the new percept tells the agent the hue value measurement $(350,360)$. We defined above that this range of hue values of cups corresponds to the colour "red". Apparently, percept $\pi_{4}$ contradicts the symbolic information on the colour of object $A$. Hence, the first hypothesis with the correspondence $\left(A, \pi_{3}\right)$ conflicts with this percept and must be disregarded in the next situation. However, percept $\pi_{4}$ conforms to the

\footnotetext{
${ }^{6}$ Of course, here we use such identifiers $\pi_{i}$ that are unique.
} 
second hypothesis with the correspondence $\left(B, \pi_{3}\right)$, which now can be updated with the new perceptual information and which affords to be taken over to the next situation, yielding the correspondence $\left(B, \pi_{4}\right)$.

The above-mentioned, fundamental concept of knowledge update is provided by the standard knowledge update axiom of the Fluent Calculus (cf. Section 2.4). The core axiom of our symbolanchoring approach is a specific instance of the general knowledge update axiom, designed and restricted precisely to perform symbol anchoring in accordance with the fundamental concepts of our approach. Employing knowledge states for representing symbol anchoring hypotheses allows us to make use of the whole reasoning capacity of the Fluent Calculus. This integration turns our approach to the symbol anchoring problem into a highly expressive and powerful theory in terms of reasoning about knowledge.

The symbol anchoring of all earlier versions of the approach of Saffiotti et al. was focused on a single object symbol, i.e., the aim of a symbol anchoring action or functionality was to determine and update the anchor for a given object identifier (cf. Section 2.3.5.1). Only the approach in [Broxvall et al., 2005] took some specific mutual dependencies among object symbols and percepts into account explicitly and introduced constraints to this end. As a result, their approach should determine the different possible combinations of object symbols and percepts, i.e. a basic hypothesis space. It is not clear, how multiple hypotheses are progressed and maintained from situation to situation individually in their approach. Yet anchoring was focused on an individual object symbol and neglects a number of important domain constraints. We will present below, how our approach takes these aspects into consideration and why they are important.

\subsection{Object Recognition System}

Recalling from Section 2.3.1, the general aim of symbol anchoring is to enable a cognitive agent to determine and to maintain the correspondence between object identifiers denoting objects of the world and perceptual images of such objects, both referring to the same object of the world. In other words, the robot shall be enabled to correctly identify an individual object each time it is perceived. Apparently, this task becomes the easier the smaller the perceptual differences between successive measurements of the same physical object are and the more different the considered object appears with respect to all other objects, i.e., the degree of difficulty of identifying an object depends on its degree of distinctness.

Some advanced, natural cognitive systems, e. g. human vision, utilize the distinctness of objects by means of a mechanism called categorical perception [Harnad, 2002]. Instead of starting the object identification from scratch upon each new measurement, object identification can take advantage of maintaining a spatial reference to the object in question within the current perceptual range of the sensor at hand. The spatial reference represents a positional estimate of the perceptual image of the physical object and eases the task to reacquire the perceptual image of the same object within the subsequent measurements, especially if the difference between successive measurements is small due to a high frame rate, a static environment or little movement of the sensor and object. This process is called object tracking (e.g. [Schulz et al., 2001]), while the problem of detecting whether and where some known objects are present in the given measurement is called object recognition (e.g. [Murase and Nayar, 1995]).

Because object recognition and tracking demand for a complex analysis of sensorimotor data and the implementation of high-fidelity object models, the deliberative, symbolic level is not suited for these tasks. For this purpose our approach assumes an object recognition and tracking system (called recognition system for short in the sequel) to be available in the cognitive robot architecture, which detects and tracks objects of the given kind robustly and continuously over time. The robustness required in our setting regards the capacity of the recognition system to not yield objects of a different kind than requested. This is because our approach can cope much better with situations wherein the percept of an object is simply not reported due to insufficient certainty of the recognition system than with situations wherein two different kinds of objects 
are mixed up and where the recognition system reported a percept as being of the wrong kind. The reason is that perceptual seemings are not taken into account in our approach as stated in Section 3.1.

Most important for our approach is that the recognition system solves the so-called data association problem robustly on its own as long as possible, i. e., the recognition system should try to track a physical object correctly over time while reporting that it is still observing the same physical object. This feature counts among the typical requirements for object tracking systems, e. g. in [Correll et al., 2006], as well as for symbol anchoring approaches, e.g. in [Bonarini et al., 2001a]. This setting stresses the distribution of functionalities nicely: The object recognition system performs detection, segmentation and categorization of sensory data, the tracking system performs short-term tracking of objects, while the task of the symbol anchoring system is to achieve object identification.

The definition of the interface between our symbol anchoring approach and the recognition system is designed to offer the desired functionalities and yet to be concise. In general, a single high-level object recognition action may yield multiple matching percepts. Each call of a sensing action that the deliberative level of the cognitive robot makes to the object recognition system includes a parameter describing the object category in question in order to allow for model-based, focused object recognition. In turn, the recognition system is expected to yield a set of current percepts of the kind given by the object category. We assume that the system returns percepts of all objects in the robot's current perceptual range that certainly fit to the given object model. Of course, the number of percepts reported by the recognition system varies and in particular may be zero.

Each percept bears a unique identifier of the sort PERCID, where we simply use the natural numbers for this sort, PERCID $=\mathbb{N}$ (cf. Section 3.4). The advantages of using natural numbers for identifiers are the availability of an unlimited number of them and the simplicity of determining the next available, unique identifier by simply increasing a counter. Given that the recognition system solves the data association problem and tracks an object as long as possible but only as long as it is certain, the system is supposed to report the same percept identifier during successful tracking of the object, and to select a fresh, unique identifier for a new percept that cannot be associated with a previous percept with certainty.

Furthermore, the recognition system shall provide an abstract description of each percept in terms of the object's phenomenal characteristics. For this purpose we adopt the representation of uncertainty and its semantics as described in Section 3.5 above. Accordingly, a percept description includes a range of one-dimensional values $(\mathbb{R} \times \mathbb{R})$ for each perceptual attribute measured, which is called a perceptual signature. The meaning of such a range of perceptual values is that some values inside the given range have been measured and values outside the given range can be excluded, i.e., a perceptual range of attribute values represents the measurements as well as the perceptual uncertainty involved. The object recognition system is responsible for measuring the perceptual attributes in question and the uncertainty involved in the measurements. Our approach assumes that the system never underestimates the uncertainty, because the bounds of an interval are utilized as constraints. Note that not all known perceptual attributes can be measured at once in a single sensing action in general. Hence, a percept comprises just the information on those attributes that have been actually measured with a certain sensing action.

As explained above, our approach requires the data association problem to be solved inside the object recognition system. This requirement is also related to the restriction of our symbol anchoring approach to whole physical objects, i. e., objects that are not composite, in accordance with Section 2.3.3.5 and our definition of a percept in Section 2.3.2.

The abstractness of the above-mentioned perceptual signatures being reported by the recognition system entails the assumption that the perceptual information is invariant in general regarding environmental influences like illumination or the sensor's position relative to the object etc. (cf. Section 2.3.3.4). Although the invariance of percepts, which is also called the object constancy problem [Vogt, 2003], may be difficult to realize for real-world recognition systems, it is an accepted assumption of formal symbol anchoring approaches (cf. [Bloch and Saffiotti, 2001]). Currently, complex object recognition systems and domain knowledge are used in order to compute such 
abstract, invariant object descriptions, while research about so-called smart sensors may provide suitable, compact sensor devices in the future (cf. e. g. [Bonarini et al., 2001a]).

In summary, our abstract definition of the interface between the object recognition system and symbol anchoring as explained above can be considered a trade-off between the invariant, omniscient perspective, that no single, autonomous robot can achieve, and a precise world model comprising any conceivable detail, or in other words the trade-off between the so-called transduction problem and the representation and reasoning problem [Wooldridge and Jennings, 1995]. 


\section{Chapter 4}

\section{Uni-Modal Symbol Anchoring}

In this chapter we define and explain the uni-modal version of our approach to the symbol anchoring problem. This approach should be considered as a particular solution to the symbol anchoring problem covering all functional requirements described in Section 2.3.4. However, this approach bears one limitation: uni-modal perception. Here, uni-modal perception refers to the limitation of a high-level sensing action to yield no more than a single percept per real-world object. Nevertheless, a percept may include information on multiple perceptual attributes at once. The restriction to the uni-modal case of sensing typically corresponds to a cognitive, robotic architecture where only a single sensor generates single percepts per object, or where sensor fusion combines the perceptual information obtained from all sensors into a single aggregate data structure. The uni-modal case avoids the additional complexity of associating multiple, individual percepts with the same object of the environment after sensing. This restricted version allowed us to develop an approach that is easier to explain and to understand, yet it implements the above-mentioned requirements of the symbol anchoring problem (cf. Section 2.3.4).

The next Chapter 5 will revoke the uni-modal limitation and will introduce our multi-modal approach along with the proof that the uni-modal version actually is a special case of the multimodal version. Both the uni-modal and the multi-modal approaches to symbol anchoring are based on the common assumptions made in Chapter 3, thus sharing the same foundation.

\subsection{Representation and Meaning}

We begin with the representation and interpretation of a percept in our approach. A percept stores the perceptual information obtained from the object recognition and tracking system as a result of executing a particular sensing action in a certain situation.

\subsubsection{Percept}

Our uni-modal approach to symbol anchoring defines a percept as a structured aggregate of perceptual information of a single physical object obtained from some sensing action, i. e., from the object recognition system. The uni-modal case of perception presupposes a one-to-one relation between object symbols and percepts in any situation. A percept is represented using the predicate $P$ with the following definition:

$$
P: \text { PERCID } \times \text { OBJCAT } \times \text { PERCSIG } \times \text { SIT }
$$

whereof its components mean the following.

1. The first parameter comprises a unique identifier of the sort PERCID standing for an individual percept. The object recognition system itself assigns the identifier to the percept in accordance with the assumptions of Section 3.11. 
2. The category of the perceived object is of sort OBJCAT. As pointed out in Section 3.6, the sort OBJCAT consists of constant symbols denoting the object categories that the cognitive robot knows and which can be perceived by the object recognition system. Given the particular object category in question as a parameter of a sensing action, the recognition system shall focus its attention to this category, e. g. by selecting the corresponding object model, and then shall return suitable percepts. Therefore, the deliberative level may assume that the percept is of the given category. In other words, object categorization of percepts is to be performed on the sensorimotor level and not on the symbolic level.

3. The perceptual signature embodies the perceptual information measured of a certain physical object. The perceptual signature associates attributes with measurements. More precisely, it is defined as a set of tuples where each tuple consists of an attribute name and an uncertainty interval of perceptual values for the corresponding attribute:

$$
\text { PERCSIG } \stackrel{\text { def }}{=} \mathcal{P}((\text { ATTR } \times(\mathbb{R} \times \mathbb{R}))
$$

Note that the perceptual signature only includes values for a subset of all known perceptual attributes in general. Thus, our approach does not assume that a sensing action always yields perceptual information on all available attributes, taking incomplete knowledge of the cognitive robot into account. This has been pointed out to be an important characteristic of any approach to the symbol anchoring problem in Section 2.3.3.2.

The uncertainty interval of the perceptual signature, $(\mathbb{R} \times \mathbb{R})$, implements our concept and meaning of representing perceptual uncertainty as explicated in Section 3.5. To recapitulate briefly, the perceptual interval is assumed to cover any perceptual uncertainty involved in the measurement process, such that values outside of this interval may be ruled out rightly.

4. The last component of the predicate $P$ denotes the situation in which the percept predicate holds with a particular instantiation. It is important to note that a percept predicate holding in a certain situation $s$ infers that it holds likewise in all states $z$ that are associated with the situation in virtue of the predicate KState $(s, z)$. By definition, being dependent on the situation parameter, a percept only holds in the specified situation right after the execution of the sensing action.

Obviously, a sensing action must have been called in the situation directly preceding situation $s$, for a percept to be available in situation $s$. In order to achieve the desired meaning of the percept predicate $P$ and the desired consistency of the formulas of our approach with respect to this predicate, we have to pose the following constraints. First, any percept is only useful if it carries some perceptual information, i.e., if its perceptual signature is a non-empty set:

$$
(\forall)(P(\pi, o, \Gamma, s) \supset \Gamma \neq \emptyset)
$$

Assuming that the lower bound of perceptual intervals inside the perceptual signature comes first in the tuple, the formulas will become easier:

$$
(\forall)(P(\pi, o, \Gamma, s) \wedge(a,(b, t)) \in \Gamma \supset b<t)
$$

This formula states that a composite tuple $(a,(b, t))$ is an element of the perceptual signature $\Gamma$ : PERCSIG of a given percept and denotes the interval of perceptual values $(b, t)$ for the attribute $a$. Hereof $b$ and $t$ stand for the lower (bottom) and upper (top) boundaries of the interval, respectively.

Our approach assumes uni-modal and one-dimensional value distributions of perceptual attributes in accordance with the concept of grounding of perceptual predicates (cf. Section 3.6) and with the interface definition to the object recognition system (cf. Section 3.11). Uni-modal value distributions of perceptual attributes seem to be sufficient, especially because we disregard anchoring of composite objects (cf. Section 2.3.3.5). Even complex perceptual attributes such as the colour of an object can be represented as multiple uni-modal distributions if necessary, e.g. 
hue, saturation and value with respect to the HSV colour model. We formalize this requirement as follows:

$$
(\forall)\left(P(\pi, o, \Gamma, s) \wedge\left(a,\left(b_{1}, t_{1}\right)\right) \in \Gamma \wedge\left(a,\left(b_{2}, t_{2}\right)\right) \in \Gamma \supset b_{1}=b_{2} \wedge t_{1}=t_{2}\right)
$$

Note that the single modality of perceptual value distributions also regards the multi-modal version of our approach designed for multiple, different sensors described in Chapter 5. The uniqueness of the percept identifier only applies within the same situation, because the object recognition system is asked to track an object for as long as possible and to report the successful tracking of an object by means of reusing the same identifier again (cf. Section 3.11). Therefore, we pose the following domain requirement:

$$
(\forall)\left(P\left(\pi, o_{1}, \Gamma_{1}, s\right) \wedge P\left(\pi, o_{2}, \Gamma_{2}, s\right) \supset o_{1}=o_{2} \wedge \Gamma_{1}=\Gamma_{2}\right)
$$

Percepts in the form of predicates $P$ on the deliberative level are the result of non-deterministic sensing actions in the dynamic environment of the cognitive robot. The number of perceivable objects cannot be anticipated in real-world applications in general. Therefore, our approach is designed to handle an arbitrary number of available percepts dynamically. For this purpose, the function NoP returns the number of percepts which are available in the given situation:

$$
\text { NoP : SIT } \mapsto \mathbb{N}
$$

This function is named after "number of percepts". $N o P(s)=k$ represents that there exists a certain number $k \geq 0$ after executing the sensing action, which specifies how many percepts have been reported by the recognition system. Thus, there exist $k$ distinct percepts in any situation such that:

$$
(\forall s)(\exists ! k, o)\left(N o P(s)=k \wedge(\exists)\left(P\left(\pi_{1}, o, \Gamma_{1}, s\right) \wedge \cdots \wedge P\left(\pi_{k}, o, \Gamma_{k}, s\right) \wedge \bigwedge_{i=1}^{k-1} \bigwedge_{j=i+1}^{k} \pi_{i} \neq \pi_{j}\right)\right)
$$

In accordance with the interface between the symbol anchoring and the recognition systems defined in Section 3.11 all percepts are of the same kind: the category $o$ of objects. ${ }^{1}$ The final conjunct of Formula (4.7) defines that there exist exactly $k$ percepts that bear distinct identifiers conforming to the Formula (4.6).

As a brief conclusion, the above representation of perceptual information takes uncertainty involved in the perception of objects into account explicitly. The meaning of and motivation for this representation have been described in detail in Section 3.5. There you can also find comparisons with the uncertainty representation of other approaches in the literature.

\subsubsection{Correspondences and Anchors}

Objects used in our symbol anchoring approach may take arbitrary names. Those objects that are known initially take identifiers of the sort $\mathcal{X}$. Later, the cognitive robot may encounter new objects in its environment, each of which is assigned an individual, fresh object identifier of the sort PERCID. For the sake of simplicity, these object identifiers of the sort PERCID coincide with the identifiers of the corresponding, original percepts. Any object identifier maintained by the symbol anchoring system is available on the whole deliberative level, of course, but their assignment must not be manipulated except by the symbol anchoring system itself.

A so-called anchor represents the correspondence between an object on the deliberative level and a percept on the sensorimotor level. Our approach defines an anchor as the fluent $A$ with:

$$
A: \mathcal{X} \cup \text { PERCID } \times \text { OBJCAT } \times \text { PERCSIG } \times \text { PERCID } \cup\{\perp\} \mapsto \text { FLUENT }
$$

The components of the fluent $A$ representing an anchor are as follows.

\footnotetext{
${ }^{1}$ This is not strictly necessary and it may be relaxed in future follow-ups (cf. Formula 4.6).
} 
1. Representing the object identifier, the first component of the fluent $A$ is a unique constant symbol of the sort $\mathcal{X} \cup$ PERCID. Because object identifiers have to be unique, our approach requires:

$$
\mathcal{X} \cap \operatorname{PERCID}=\emptyset
$$

2. The second component of fluent $A$ denotes the category of the object at hand and is of sort OBJCAT. If the object is known from the initial situation on, its category is known, too (cf. Formula (4.11)). Along with each percept, the object recognition system reports the percept's object category and assigns an identifier of sort PERCID. In our current approach, the object category remains static for any given object at runtime. The rationale and motivation behind using object categories as well as a comparison with other approaches in the literature can be found in Section 3.3.

3. The third component of the anchor fluent $A$ is of sort PERCSIG and has a slightly different meaning compared to the perceptual signature of the percept predicate $P$. Regarding the anchor fluent, it represents the current best estimate of perceptual information about the object associated with the fluent $A$ at hand. Since no action has been executed until the initial situation $S_{0}$ - particularly no sensing action - there is no perceptual information available about any object in $S_{0}$; hence the perceptual estimate of all object anchors is empty initially. The perceptual estimate of a certain anchor is refined stepwise upon obtaining a new percept corresponding to the anchor at hand. We will explicate the method of refining this component below (cf. Section 4.2.3). In general terms the current best perceptual estimate serves as an aggregate structure of all previous perceptual information learned about the associated object so far to be used by all actions that require such information. Most notably our symbol anchoring approach integrates all percepts corresponding to the anchor into this sole representation, which remains available at all times - even when a percept ceased to hold later. To this end, we will define a basic information fusion process below. Recall that percepts are the only source of information for this anchor component, the perceptual estimate, reported by the recognition system, which is due to our concept of independence of information of the deliberative and sensorimotor levels (cf. Section 3.1).

Similar to the uncertainty interval representation of the percept predicate $P$ (cf. Formula (4.1)), the best perceptual estimate of an anchor takes uncertainty involved in the perception of objects into account explicitly. The capacity to represent perceptual uncertainty counts among the important criteria of an approach to the symbol anchoring problem as described in Section 2.3.3.1. Our approach utilizes a basic, and yet powerful means to this end. In comparison with other pieces of work, the representation of perceptual uncertainty chosen in the Fuzzy logic approach of Coradeschi et al. [2001] is essentially the same as in our approach (cf. Section 2.3.5.2). Other approaches of Saffiotti's group do not maintain and represent perceptual uncertainty explicitly as has been described in Section 3.5. The best perceptual estimate is used with a very similar meaning in the approaches of Saffiotti's group that are derived from the basic approach described in Section 2.3.5.1 as well as in the approach based on conceptual spaces (cf. Section 2.3.5.3).

4. The fourth component of type PERCID $\cup\{\perp\}$ of the anchor fluent $A$ embodies the correspondence with a particular percept. The percept identifier of the most recent percept associated with the object symbol is stored here. Initially, the special constant $\perp$ meaning "undefined" is assigned to this parameter for all anchor fluents, i.e., the corresponding physical object has not yet been perceived. The percept identifier stored in this component persists even though the corresponding percept predicate may have ceased to hold in the meantime, because the recognition system might be able to track the corresponding physical object while the deliberative level proceeds to the next situation. In other words, a percept identifier stored in this component of the anchor fluent may recur or not, depending on whether the recognition system is able to track the considered physical object. Sections 3.4 and 3.11 described the assignment of percept identifiers in detail. 
Together the object and percept identifiers recorded in the anchor fluent represent the correspondence between the symbol denoting a certain object on the deliberative level and the perceptual image measured of the supposedly corresponding physical object. The Figure 2.1 on page 13 illustrates this link.

The Signature (4.8) of anchor fluent $A$ includes object identifiers of type $\mathcal{X} \cup$ PERCID. The individual functionalities, to be introduced below, require the following initial configuration of the anchors regarding object identifiers. Anchor fluents standing for objects with identifiers of sort PERCID must not occur in the initial situation $S_{0}$, because they will only be introduced afterwards:

$$
(\forall z, x: \text { PERCID })\left(K \operatorname{Ktate}\left(S_{0}, z\right) \supset \neg(\exists o, \Gamma, \pi)(\operatorname{Holds}(A(x, o, \Gamma, \pi), z))\right)
$$

Objects with identifiers of sort $\mathcal{X}$ are all supposed to be known initially. For each one of them our approach requires an empty anchor fluent to exist in situation $S_{0}$ :

$$
(\forall z, x: \mathcal{X})\left(\operatorname{KState}\left(S_{0}, z\right) \supset(\exists ! o, \Gamma, \pi)(\operatorname{Holds}(A(x, o, \Gamma, \pi), z) \wedge \Gamma=\emptyset \wedge \pi=\perp)\right)
$$

Note that Formula (4.11) specifies the uniqueness of anchor fluents $A$ for objects $x: \mathcal{X}$ with respect to individual, possible states $z$ in the initial situation $S_{0}$. Moreover, the requirement of anchor fluents to be unique also has to hold for all object symbols $x:(\mathcal{X} \cup$ PERCID) for all states associated with any subsequent situation. To this end the predicate Consistent : STATE defines when a given state $z$ is considered consistent with respect to uniqueness of identifiers in particular:

$$
\begin{aligned}
\text { Consistent }(z) \equiv & \left(\operatorname{Holds}\left(A\left(x, o_{1}, \Gamma_{1}, \pi_{1}\right), z\right) \wedge \operatorname{Holds}\left(A\left(x, o_{2}, \Gamma_{2}, \pi_{2}\right), z\right)\right. \\
& \left.\supset o_{1}=o_{2} \wedge \Gamma_{1}=\Gamma_{2} \wedge \pi_{1}=\pi_{2}\right) \wedge \\
& \left(\text { Holds }\left(A\left(x_{1}, o_{1}, \Gamma_{1}, \pi\right), z\right) \wedge \operatorname{Holds}\left(A\left(x_{2}, o_{2}, \Gamma_{2}, \pi\right), z\right) \wedge \pi \neq \perp\right. \\
& \left.\supset x_{1}=x_{2} \wedge \Gamma_{1}=\Gamma_{2} \wedge o_{1}=o_{2}\right)
\end{aligned}
$$

The predicate Consistent holds true iff the given state $z$ is considered consistent. According to the Definition (4.12) this is the case iff each anchor fluent inside the state $z$ is unique with respect to both object identifiers and percept identifiers. Since multiple anchor fluents may have no correspondence with a percept in the same possible state, especially in the initial situation $S_{0}$ (cf. Formula (4.11)), the second conjunct of predicate Consistent only regards anchors which $\pi \neq \perp$ holds for.

We employ the predicate Consistent especially for verifying the consistency of symbol anchoring hypotheses, where the mutual dependencies between percept and symbol identifiers have to be checked. For example, each of the following hypotheses $z_{i}$ of correspondences is inconsistent with respect to the predicate Consistent:

$$
\begin{aligned}
& z_{1}=A\left(C_{1}, O, \Gamma_{1}, N_{1}\right) \circ A\left(C_{2}, O, \Gamma_{1}, N_{1}\right) \circ z_{1 a} \\
& z_{2}=A\left(C_{1}, O, \Gamma_{1}, N_{1}\right) \circ A\left(C_{1}, O, \Gamma_{2}, N_{2}\right) \circ z_{2 a} \\
& z_{3}=A\left(C_{1}, O, \Gamma_{2}, N_{2}\right) \circ A\left(C_{2}, O, \Gamma_{2}, N_{2}\right) \circ z_{3 a}
\end{aligned}
$$

such that $\not \models$ Consistent $\left(z_{1}\right)$ and $\not \models$ Consistent $\left(z_{2}\right)$ and $\not \models$ Consistent $\left(z_{3}\right)$.

Our uni-modal symbol anchoring approach assumes all initial hypotheses to be consistent with respect to the predicate Consistent:

$$
(\forall z)\left(\operatorname{KState}\left(S_{0}, z\right) \supset \text { Consistent }(z)\right)
$$

The above consistency constraints altogether serve to rule out any inappropriate hypothesis automatically, thanks to the strong expressiveness of our symbol anchoring approach. We count this as one of the most appealing features and advantages of formal approaches to the symbol anchoring problem in general.

The following cognitive interpretation sustains the definition of predicate Consistent intuitively. On the one hand, the cognitive robot may choose to denote two percepts using the same 
identifier. But this requires that both percepts are actually the same or refer to the same physical object, because the physical world represents the grounding truth for the robot's world models. The reason can be found in the fundamental concept of philosophy, which our approach complies with: "seeing is normally believing" [Bell and Huang, 1999, p. 1]. ${ }^{2}$ On the other hand, the second conjunct of predicate Consistent exhibits that two objects have to be named equally if the real-world referents are actually the same. Likewise, this interpretation is based on the concept that the real world naturally represents the referent, which any world model has to accommodate. The two conjuncts of the definition of predicate Consistent can be considered a top-down and a bottom-up constraint on anchoring object symbols to percepts. Bidirectional relations between symbolic and perceptual levels are considered important for an approach to symbol anchoring (cf. Section 2.3.3).

Being one component of the anchor fluent $A$, the best perceptual estimate explicitly represents perceptual uncertainty in accordance with the method proposed in Section 2.3.3.1. Below you will see, how this representation is maintained over time. Apparently, the anchor fluent accounts for incomplete knowledge regarding unavailable correspondences between object symbols and percepts, e. g., no correspondence exists initially. New object identifiers and associated anchor fluents may be introduced on demand during runtime in order to allow for dynamic, non-deterministic environments, wherein the cognitive robot may experience yet unknown instances of objects. The sort PERCID is reserved solely for new object symbols in order to take this aspect of incomplete knowledge into account.

Most other approaches in the literature utilize a data structure called anchor for representing the correspondences between object symbols and percepts, in particular those of Saffiotti et al. (cf. Section 2.3.5). Coradeschi and Saffiotti [2001] additionally included a set of perceptual properties in the anchor, that need to be known by the cognitive robot in order to be able to act on the object. In contrast to their approach, the domain axiomatization of an application based on our approach includes so-called action precondition axioms for defining the preconditions required to hold in order for the robot to be able to execute the intended actions. This way, the Fluent Calculus, which our approach is based on, provides a much more flexible and expressive means to stipulate the preconditions of actions precisely (cf. Section 2.4). Due to a rather procedural planning system, the various approaches of Saffiotti et al. do not specify domain constraints on the consistency of the representation, except for the approach of [Broxvall et al., 2005] implementing a few, rather limited constraints.

\subsubsection{Representing Multiple Hypotheses}

In order to handle ambiguous situations and incomplete knowledge appropriately, we pointed out that an approach to the symbol anchoring problem shall be able to represent and to reason about multiple, possible states (cf. Section 3.10). Our approach in particular aims at minimizing the ambiguity using our concept of maintaining all possible states of symbol anchoring correspondences that the cognitive robot thinks it and its environment are in. In the above Sections 3.9 and 3.10 we have defined the term and concept of a symbol anchoring hypothesis. Also, we have explained in principle how our approach maintains multiple hypotheses simultaneously.

To recapitulate, the predicate KState $:$ SIT $\times$ STATE allows to associate a situation with multiple, possible states in the Fluent Calculus (cf. Section 2.4). In our approach all anchors of all known objects are present in all possible states associated with the initial situation (cf. Formula (4.11)). Then, these anchors will be carried forward to subsequent situations according to the formulas that will be defined below.

Example 4.1 The following basic example illustrates the representation of symbol anchoring correspondences between object symbols $C_{1}, C_{2}$ and percept identifiers $N_{1}$ and $N_{2}$. Here, two explicit, alternative hypotheses are specified utilizing a knowledge state for a certain situation $S$ :

\footnotetext{
${ }^{2}$ Note that our approach excludes so-called perceptual seemings (cf. Section 3.1).
} 


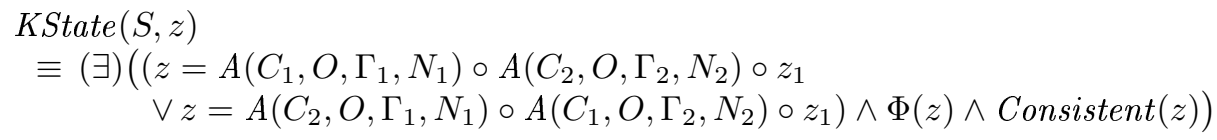

This formula specifies the knowledge state for the situation $S$, wherein only the two objects $C_{1}$ and $C_{2}$ are known with respect to symbol anchoring. The first possible state embodies the correspondences of object $C_{1}$ with percept $N_{1}$ and of object $C_{2}$ with percept $N_{2}$, while the state represented in the second disjunct interchanges the object identifiers of the correspondences of the same two objects. Besides those two symbol anchoring hypotheses, the predicate Consistent disallows further alternative hypotheses regarding the objects $C_{1}$ and $C_{2}$, although the Formula (4.14) stipulates the knowledge state incompletely due to $z_{1}$. The state formula $\Phi(z)$ may specify further symbolic information available in the knowledge state.

It is important to note that the same percept identifier always refers to the very same physical object in a given situation regardless which hypothesis is considered, because the percept predicate $P$ holds likewise in the whole situation (cf. Section 4.1.1). Also note the relation between the best perceptual estimate and the percept identifier. Due to our concept of independence of perceptual and symbolic information, the best perceptual estimates, $\Gamma_{1}$ and $\Gamma_{2}$ in the above Example 4.1, are updated solely from perceptual information (cf. Section 3.1). Accordingly, $\Gamma_{1}$ is associated with $N_{1}$ and $\Gamma_{2}$ with $N_{2}$ in this example regardless of the correspondence with the object symbol. This concept is sustained by the fact that percepts-together with the information they contain in terms of perceptual attributes of the observed, respective object-hold in the whole situation, i.e., percepts are independent of individual hypotheses.

We certainly agree with Coradeschi and Saffiotti [2002] that an appropriate approach to the symbol anchoring problem should provide the capacity to represent and to reason about multiple hypotheses of correspondences (cf. Section 2.3.4). This capacity forms a crucial prerequisite for a cognitive robot to handle ambiguous situations robustly with respect to recognizing objects in its dynamic environment. To our knowledge, no other approach of similarly rich logical expressiveness exists regarding multiple symbol anchoring hypotheses including arbitrary, global and individual constraints and incomplete knowledge such as our approach affords.

\subsection{Computing the Space of Hypotheses}

Our symbol anchoring approach employs a so-called knowledge update axiom for determining and maintaining the correspondences between percepts and object symbols. Recall that a knowledge update axiom takes a world model of a certain situation and updates the world model according to new sensory data and the effects of the action performed, in general (cf. Section 2.4). More precisely, given the knowledge state $\operatorname{KState}(s, z)$ of a certain situation $s$ the knowledge update axiom determines the knowledge state $\operatorname{KState}\left(D o(a, s), z^{\prime}\right)$ of the successor situation $D o(a, s)$ after performing action $a$ in situation $s$. The knowledge state corresponding to the successor situation has to comply with all currently available perceptual data.

We adapted the general technique of knowledge update to the specific task of symbol anchoring as follows. Symbol anchoring is performed by the special epistemic action AnchorU defined as: ${ }^{3}$

$$
\text { Anchor } U \text { : OBJCAT } \mapsto \text { ACTION }
$$

Given the current hypothesis space of correspondences between object symbols and percepts in a certain situation $s$ and given the current percepts resulting from a sensing action, the knowledge update axiom of the uni-modal symbol anchoring action Anchor $U(o)$ defines the hypothesis space

\footnotetext{
${ }^{3}$ The name of the epistemic action AnchorU stands for "to anchor" an object using "uni-modal perception".
} 
of correspondences of the successor situation $s^{\prime}=\operatorname{Do}(\operatorname{Anchor} U(o), s)$ as follows:

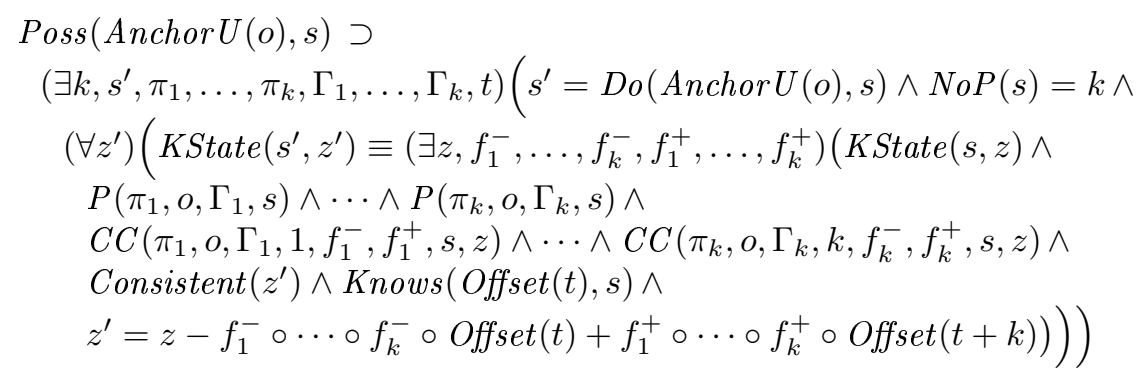

In order to account for unpredictable, dynamic environments, this knowledge update Axiom (4.15) for symbol anchoring is designed in the form of a schematic formula depending on the number $k$ of available percepts. The natural number $k$ is determined by function $N o P$ and may take values among $k \geq 0$ (cf. Section 4.1.1). Accordingly, the schematic Axiom (4.15) could be interpreted as if there exists one instantiated axiom for each possible value of $k$. In the particular case $k=0$, meaning there is no percept available, the knowledge update Axiom (4.15) for symbol anchoring reduces from (4.15) to the following simple axiom:

$$
\begin{aligned}
& \operatorname{Poss}(\operatorname{AnchorU}(o), s) \supset \\
& \left(\exists s^{\prime}, t\right)\left(s^{\prime}=\operatorname{Do}(\operatorname{Anchor} U(o), s) \wedge N o P(s)=0 \wedge\right. \\
& \left.\quad\left(\forall z^{\prime}\right)\left(\operatorname{KState}\left(s^{\prime}, z^{\prime}\right) \equiv(\exists z)\left(\operatorname{KState}(s, z) \wedge \text { Consistent }\left(z^{\prime}\right) \wedge z^{\prime}=z\right)\right)\right)
\end{aligned}
$$

Basically, the Axiom (4.16) expresses that all the hypotheses of correspondences present in the knowledge state of situation $s$ will not change at all when performing symbol anchoring in case there is no percept available. The general knowledge update Axiom (4.15) for symbol anchoring takes one parameter: The object category $o$ : OBJCAT is what both object recognition and symbol anchoring focus on according to the fundamental concepts of our approach described in Sections 3.3 and 3.4. Most notably, the Axiom (4.15) anchors all available percepts of the specified object category at once.

Next we are going to define and explain each component of the above axiom in detail. Apparently, the knowledge update Axiom (4.15) for symbol anchoring is only applicable if the preconditions are fulfilled, i. e., if Poss $(\operatorname{Anchor} U(o), s)$ holds. Although it only makes sense to perform symbol anchoring if object symbols need to be anchored and if relevant percepts are available, symbol anchoring is always applicable in principle:

$$
(\forall)(\operatorname{Poss}(\operatorname{Anchor} U(o), s)
$$

The fundamental, interrelated concepts of the symbol anchoring Axiom (4.15) are the following:

1. There are exactly $k$ distinct percepts available. Therefore, the axiom includes $k$ instances of each of the following: of percept predicate $P$, of predicate $C C$, of fluent $f^{-}$and of fluent $f^{+}$. Since the symbol anchoring Axiom (4.15) has to analyse all percepts currently available, it incorporates the Constraint (4.7) partly stating that there are exactly $k$ distinct percepts available, which is determined by $N o P(s)=k$. It is very important to note that all available percepts are taken into account at once-no matter how many are available — with the result of a grounded correspondence of each percept with some object symbol right after applying this symbol anchoring axiom.

2. For each percept $\pi_{1}, \ldots, \pi_{k}$ of the given situation an individual instance of predicate $C C$ is responsible for choosing an appropriate correspondence for that percept. The result of predicate $C C$ is the new correspondence for the considered percept $\pi_{i}$, which is assigned to the new anchor fluent $f_{i}^{+}$, while $f_{i}^{-}$holds the outdated anchor fluent, for $i=0 \ldots k$. The predicate $C C$ may be true for multiple correspondences non-deterministically. As you will see in its definition, this predicate always finds at least one possible correspondence, i. e., it never fails, when used in the appropriate way as in Axiom (4.15). 
3. The symbol anchoring axiom generates a new hypothesis of correspondences $z^{\prime}$ from a previous hypothesis $z$ by subtracting all outdated anchor fluents $f_{1}^{-}, \ldots, f_{k}^{-}$and adding all updated anchor fluents $f_{1}^{+}, \ldots, f_{k}^{+}$. It is very important to note that a knowledge update axiom in general and the symbol anchoring Axiom (4.15) in particular consider all possible hypotheses $z$ associated with situation $s$ by $\operatorname{KState}(s, z)$ at once, because the axiom implicitly defines precisely how each hypothesis $z$ may be progressed to the next situation $s^{\prime}$, no matter how many instantiations of $z$ there are in situation $s$. Similarly, given KState $(s, z)$ the symbol anchoring Axiom (4.15) also considers all possible successor hypotheses $z^{\prime}$ for each original hypothesis $z$ and defines precisely how each successor hypothesis $z^{\prime}$ has to be computed based on $z$. In general, between one to many original hypotheses $z$ may be associated with a certain situation $s$, and for each of them the Axiom (4.15) may determine between zero to many successive hypotheses $z^{\prime}$. The complexity of the hypothesis space of the successor situation $s^{\prime}$ will be explicated in Section 4.2.5.

4. Each new hypothesis $z^{\prime}$ has to be consistent with respect to all its correspondences altogether. The predicate Consistent $\left(z^{\prime}\right)$ makes sure that only those successor hypotheses are taken into account which satisfy the mutual dependencies among individual correspondences of the same hypothesis as defined in Formula (4.12). Thus, even though the conjunction of $k$ instances of the non-deterministic predicate $C C$ may yield a combination of all possible correspondences, the predicate Consistent cleans the new hypothesis space off any inappropriate hypothesis, i.e. off any invalid combination of correspondences. In comparison, the scope of predicate $C C$ is limited to all correspondences of a single hypothesis for a single percept, while the scope of predicate Consistent covers all correspondences of all percepts of the considered hypothesis.

As a conclusion, the preeminent feature of our symbol anchoring approach is to determine all possible hypotheses of all valid correspondences for all available percepts at once. Our fundamental rationale behind this concept is manifold. First, the capacity to deal with ambiguous situations appropriately represents a crucial functionality of any approach to the symbol anchoring problem (cf. Section 3.10). In our terms this requires to represent and to reason about each plausible interpretation of the state the cognitive robot is in, lest to neglect the right one accidentally. Second, our objective is to determine a picture of the state the cognitive robot and its environment are in, which is as complete as possible yet is consistent. Naturally, the more information is taken into consideration in the reasoning process, the less hints could be missed out. Conversely, verifying the consistency of hypotheses disregards implausible ones explicitly. We will include a proof that the consistency of the whole hypothesis space is preserved in our approach in the next chapter.

To our knowledge, the rich, logical expressiveness that our approach exhibits with respect to representing and reasoning about all possible hypotheses at once yet preserving their consistency is not met by other existing approaches including the ones summarized in Section 2.3.5. For instance, the approaches of Saffiotti's group mentioned above and their descendant versions only anchor the given object symbol in a top-down fashion to possibly multiple percepts [Coradeschi and Saffiotti, 2001, Broxvall et al., 2004a, 2005].

\subsubsection{Computing the Correspondences}

How are the correspondences between a given percept and an appropriate object identifier determined in our approach? This is the task of the predicate $C C$ named after "choice of correspondence" which we define in the following way:

$C C:$ PERCID $\times$ OBJCAT $\times$ PERCSIG $\times$ PERCID $\times$ FLUENT $\times$ FLUENT $\times$ SIT $\times$ STATE 
The predicate $C C$ comprises a disjunction of three symbol anchoring functionalities: ${ }^{4}$

$$
\begin{aligned}
& C C\left(\pi_{P}, o, \Gamma_{P}, n, f^{-}, f^{+}, s, z\right) \equiv \\
& \quad(\exists)\left(\Delta_{T}\left(x, o, \Gamma^{-}, \pi_{P}, \Gamma^{+}, s, z\right) \wedge f^{-}=A\left(x, o, \Gamma^{-}, \pi_{P}\right) \wedge f^{+}=A\left(x, o, \Gamma^{+}, \pi_{P}\right)\right) \\
& \vee(\exists)\left(\Delta_{F}\left(x, o, \Gamma^{-}, \pi^{-}, \pi_{P}, \Gamma^{+}, s, z\right) \wedge f^{-}=A\left(x, o, \Gamma^{-}, \pi^{-}\right) \wedge f^{+}=A\left(x, o, \Gamma^{+}, \pi_{P}\right)\right) \\
& \vee(\exists)\left(\Delta_{N}\left(x, o, \pi_{P}, n, s, z\right) \wedge f^{-}=\emptyset \wedge f^{+}=A\left(x, o, \Gamma_{P}, \pi_{P}\right)\right)
\end{aligned}
$$

Regarding the way the predicate $C C$ is used in the symbol anchoring Axiom (4.15), the parameters $\pi_{P}, o, \Gamma_{P}, n, s$ and $z$ are instantiated, i. e. ground variables, and are determined outside of $C C$, while $C C$ identifies the fluents $f^{-}$and $f^{+}$internally. Accordingly, predicate $C C$ determines an appropriate correspondence with an object symbol, deduces the new anchor fluent $f^{+}$and the related, outdated anchor fluent $f^{-}$given the following: the percept $\pi_{P}$ to be anchored, the object category $o$ considered, the perceptual signature $\Gamma_{P}$ of percept $\pi_{P}$, and the considered situation $s$ and hypothesis $z$. The definition of predicate $C C$ allows it to be satisfiable by multiple possibilities. First, each of the three disjunctive functionalities $\Delta_{T}, \Delta_{F}$ or $\Delta_{N}$ standing for "Track", "Find" and "New", respectively, may yield several possible correspondences with the given percept. Second, the functionalities may choose one of several object symbols if applicable to the correspondence with the given percept due to the existential quantification in Formula (4.18). Each of these possibilities of satisfying predicate $C C$ gives rise to a potential correspondence with the considered percept. But the above-mentioned domain constraints and consistency constraints may exclude certain correspondences from entering the new hypothesis space of the successor situation $s^{\prime}$. This way, predicate $C C$ defines the complete space of possible correspondences of the given percept, such that the symbol anchoring Axiom (4.15) determines all hypotheses of correspondences in situation $s^{\prime}$.

In accordance with the evaluation criteria proposed by Coradeschi and Saffiotti [2002], which we summarized in Section 2.3.4, our approach implements all functionalities of symbol anchoring, which will be defined and explained in detail in the next few sections, namely:

- The functionality to determine and to establish a non-existent correspondence with a physical object is realized by means of the macro $\Delta_{F}$.

- The functionality to track and to update an existing correspondence is the task of the macro $\Delta_{T}$.

- The functionality to introduce a new object symbol for a new object of the robot's environment is implemented in macro $\Delta_{N}$, although it is not listed explicitly among the required criteria of Section 2.3.4.

All three functionalities have been integrated into the predicate $C C$ (cf. Formula (4.18)).

In contrast to other approaches, especially to those of Saffiotti et al. described in Section 2.3.5, our approach smoothly integrates all three symbol anchoring functionalities. You will see below, that we defined the functionalities such that their internal preconditions determine precisely whether a certain functionality is applicable in the given situation. This way the appropriate functionality is chosen and employed automatically depending on the given situation.

\subsubsection{Tracking an Object}

The functionality "Track" of macro $\Delta_{T}$ used inside predicate $C C$ applies if the object recognition system has been able to track an object associated with the given percept identifier since the last execution of symbol anchoring successfully. ${ }^{5}$ In other words, Track applies if an anchor fluent exists with the given percept identifier, thus representing a grounded correspondence with a certain object symbol in situation $s$, and if a current percept bearing the same percept identifier has been

\footnotetext{
${ }^{4}$ We adopt the term symbol anchoring "functionality" in our work from Coradeschi and Saffiotti [2001] for the sake of readability, because their respective objectives match closely.

${ }^{5}$ Speaking precisely, "execution of symbol anchoring" in the context of our approach stands for applying the symbol anchoring Axiom (4.15) in the given situation.
} 
measured in situation $s$. Hence, we can assume that this new percept originates from the same physical object in accordance with Section 3.11, such that the corresponding anchor fluent can be updated with the new perceptual information. We define the functionality Track in form of macro $\Delta_{T}$ :

$$
\begin{aligned}
& \Delta_{T}: \mathcal{X} \cup \text { PERCID } \times \text { OBJCAT } \times \text { PERCSIG } \times \text { PERCID } \times \text { PERCSIG } \times \text { SIT } \times \text { STATE }
\end{aligned}
$$

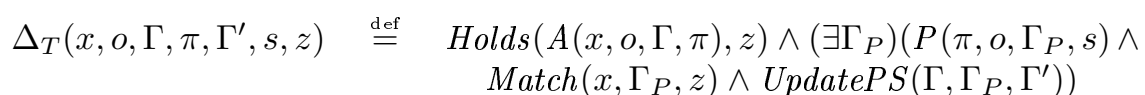

Due to the Definition (4.19) and the existential quantification of its free variables in predicate $C C$, the functionality Track applies in state $z$ and situation $s$, if there exists some object $x$, the anchor fluent corresponding to $x$ and a current percept $\pi$ such that:

1. the percept identifier of the percept predicate is the same as the one in the anchor fluent;

2. the symbolic information about object $x$ and the perceptual information about percept $\pi$ match appropriately; and

3. the perceptual estimate of the anchor fluent can be updated given the new percept.

If these conditions are satisfied, Track yields the new perceptual estimate $\Gamma^{\prime}$ to be used henceforth in the anchor fluent of object $x$. The predicate $C C$ completes the update of the considered, tracked correspondence by simply exchanging the outdated perceptual estimate of the anchor fluent for the new perceptual estimate, while all other components of the considered anchor remain the same. In case the functionality Track is applicable, the constraints of Formula (4.7) and of predicate Consistent prevent the tracked object $x$ and the percept $\pi$ from becoming involved in any alternative correspondence within the same hypothesis $z$ and the same situation $s$.

Note that the functionality Track is applicable even if considerable time has passed since the last execution of symbol anchoring, because the tracking system might have been able to track the object of interest over the whole period of time successfully even though no symbol anchoring update took place in the meantime. This case demonstrates the asynchronous and rather independent application of the recognition and tracking system on the one side and the symbol anchoring update on the other side.

Being a conjunct of macro $\Delta_{T}$, the predicate Match verifies whether the anchor of the given object matches the considered percept:

$$
\text { Match }: \mathcal{X} \cup \text { PERCID } \times \text { PERCSIG } \times \text { STATE }
$$

$$
\begin{gathered}
\operatorname{Match}\left(x, \Gamma_{P}, z\right) \equiv\left(\exists o, \Gamma_{A}, \pi\right)\left(\operatorname{Holds}\left(A\left(x, o, \Gamma_{A}, \pi\right), z\right) \wedge\right. \\
(\forall)\left(\left(\gamma_{P} \in \Gamma_{P} \wedge \gamma_{P}=\left(a, i_{P}\right) \wedge \gamma_{A} \in \Gamma_{A} \wedge \gamma_{A}=\left(a, i_{A}\right) \wedge \operatorname{AttrTypeStatic}(o, a)\right.\right. \\
\left.\supset \text { Intersect }\left(i_{P}, i_{A}\right)\right) \wedge \\
\left(\gamma_{P} \in \Gamma_{P} \wedge \gamma_{P}=\left(a, i_{P}\right) \wedge \operatorname{Holds}(\operatorname{Prop}(x, a, v), z) \wedge \operatorname{AttrTypeStatic}(o, a)\right. \\
\left.\left.\left.\supset \text { GroundingInt }\left(o, a, v, i_{P}\right)\right)\right)\right)
\end{gathered}
$$

The conditions included in the above definition of predicate Match consist of three parts, all of which have to be satisfied:

1. The predicate $\operatorname{Match}\left(x, \Gamma_{P}, z\right)$ requires an anchor fluent for the given object $x$ of the considered hypothesis $z$.

2. For those perceptual attributes which are static and which are present in both the anchor and the percept, the respective intervals have to intersect (cf. Section 3.6). Further details about this will be provided below. 
3. For those perceptual attributes which are static, which have been reported in the percept, and which the robot has some symbolic information about, the respective intervals have to match according to the grounding of perceptual predicates. The grounding of perceptual predicates has been described in Section 3.6, while macro GroundingInt will be defined below.

The Formula (4.20) defining predicate Match employs the predicate Intersect. Considering the restriction of our approach to uni-modal perceptual attributes (cf. Formula (4.5)) and the definition of the interface between our symbol anchoring approach and the object recognition system according to Section 3.11, the intersection of two perceptual intervals is a simple but appropriate means for verifying whether the best perceptual estimate agrees with the new percept. We define the predicate Intersect in Formula (4.21) to hold true for the given two real number intervals, iff the two intervals share a non-empty range of real numbers.

$$
\begin{gathered}
\text { Intersect }:(\mathbb{R} \times \mathbb{R}) \times(\mathbb{R} \times \mathbb{R}) \\
\text { Intersect }\left(\left(c_{1}, c_{2}\right),\left(b_{1}, b_{2}\right)\right) \equiv\left|c_{2}-c_{1}\right|>0 \wedge\left|b_{2}-b_{1}\right|>0 \wedge \\
\left(\left(b_{1} \leq c_{1}<b_{2}\right) \vee\left(b_{1}<c_{2} \leq b_{2}\right) \vee\left(c_{1} \leq b_{1}<c_{2}\right)\right)
\end{gathered}
$$

The predicate Match - as defined in Formula (4.20) above-employs the fluent Prop in order to reason about phenomenal features of objects on the symbolic level. This way, the fluent Prop provides a general, domain-independent interface to the symbolic information about objects (cf. Formula (3.3)).

Recall that the predicate Grounded serves the purpose of grounding of the given perceptual predicates and attributes on perceptual intervals (cf. Formula (3.1)). The predicate Match employs the new macro GroundingInt, the name of which is short for "grounding the given interval". While the predicate Grounded realizes the symbol grounding by means of a mapping to a pre-defined interval of perceptual values, the macro GroundingInt tests whether the considered percept matches the symbolic information about a given perceptual attribute of the same object. Based on predicate Grounded, we define the auxiliary macro GroundingInt as follows.

$$
\begin{aligned}
& \text { GroundingInt : OBJCAT } \times \text { ATTR } \times \text { PERCPRED } \times(\mathbb{R} \times \mathbb{R}) \\
& \text { GroundingInt }\left(o, a, p,\left(c_{1}, c_{2}\right)\right) \stackrel{\text { def }}{=} \\
& \quad(\exists)\left(\text { Grounded }\left(o, a, p,\left(b_{1}, b_{2}\right)\right) \wedge \text { Intersect }\left(\left(c_{1}, c_{2}\right),\left(b_{1}, b_{2}\right)\right)\right)
\end{aligned}
$$

Given a certain object category, the macro GroundingInt holds true, if the considered range of the measured perceptual attribute values fits the grounding of the given perceptual predicate. For similar reasons, both the predicate Match and the macro GroundingInt utilize an intersection of intervals. We consider this idea appropriate since the intervals representing vagueness of perceptual predicates meet intervals representing noise and imprecision of perception for a comparison on equal grounds. Requiring a precise, complete match of both intervals instead seemed to be too restrictive.

The predicate Match is defined to verify the symbolic information with respect to perceptual information in Formula (4.20). This match is required for static perceptual attributes only. The reason for this is part of our strategy of selecting the most plausible hypothesis of correspondences, which will be explained in Section 4.3 in detail. Basically, our symbol anchoring approach is designed to yield all potential hypotheses of all possible combinations of correspondences between object symbols and percepts, neglecting not a single potential candidate hypothesis. Thus, dynamic perceptual attributes might be utilized to favour one hypothesis for the other in a later step, but definitely not in order to rule some of them out as early as inside of the fundamental predicate Match. The predicate UpdatePS, which is also employed in the macro Track, will be described in the next section. 
Furthermore, the third conjunct of predicate Match stipulates to consider only those perceptual predicates of the symbolic knowledge about the object's properties that actually have been perceived, i.e., where the percept contains perceptual values for the corresponding attribute. This concept accounts for incomplete knowledge of the cognitive robot in accordance with the fundamental characteristics of an appropriate approach to the symbol anchoring problem (cf. Section 2.3.3.2). On the one hand, limited equipment or environmental conditions may prevent the cognitive robot from recognizing all perceptual attributes. On the other hand, a single sensing action typically yields just a subset of all perceptual attributes that could possibly be measured employing all sensing devices of the cognitive robot altogether.

As a conclusion, the macro $\Delta_{T}$ implements the symbol anchoring functionality to track and to update an anchor. This functionality is listed among the basic requirements for any approach to the symbol anchoring problem (cf. Section 2.3.4). The definition of predicate Match applies the concept of representing perceptual uncertainty of our approach: Values outside of a given perceptual interval may be excluded safely. Since the same concept of representing perceptual uncertainty is shared among all components of the representation, its meaning is preserved appropriately, although the perceptual intervals are interpreted in different ways depending on their particular use (cf. predicate $P$ vs. fluent $A$ ).

Comparing the symbol anchoring functionality Track of our approach with the corresponding implementations in the approaches of Coradeschi and Saffiotti [2001, 2002] and of Chella et al. [2004] described in Section 2.3.5, their main differences are as follows, apart from what has been mentioned above already. Our approach does not predict the perceptual signature to be used by the object recognition system in order to guide the recognition. In accordance with our interface definitions of the object recognition system, our approach assumes that tracking of an object in the continuous sensory data flow may be accomplished by the object recognition and tracking system on the sensorimotor level more appropriately and does not involve the symbol anchoring system (cf. Section 3.11). It allows our approach to be designed in the domain-independent and general way as presented here. The prediction of the perceptual properties is a highly domaindependent function and intertwines the otherwise rather separate systems of symbol anchoring, action planning, action execution and object recognition. Intertwining these systems opposes the fundamental objectives of our work.

Both our approach and the one of Coradeschi and Saffiotti [2001] similarly take incompleteness of perceptual information into account in the function of matching symbolic and perceptual information. However, Coradeschi and Saffiotti [2001] do not distinguish between dynamic and static perceptual attributes, which causes problems regarding the applicability of their approach and regarding the domain specification. In particular their function "match", defined in Formulas (2.2) and (2.3), would simply fail for dynamic perceptual attributes of an object where the robot knows symbolic information about.

\subsubsection{Finding and Reacquiring a Correspondence}

Of course, the object recognition and tracking system cannot track all objects of interest robustly over an unlimited period of time in dynamic, real environments. Cognitive robots typically have to cope with frequent obstructions of the objects of interest and noisy perception in such environments. But we do not expect the object recognition system to provide infallible tracking of objects. Rather, the recognition system and the symbol anchoring system complement each other beneficially. While some systems may easily fail to track an object upon short, partial obstructions of it, other systems may perform much better. According to the interface of our symbol anchoring approach with the object recognition system defined in Section 3.11, the failure to track the object of interest is signalled by the absence of the percept with the same identifier. If the tracking system recognizes the same object of the world later again, although it failed to recognize the object for a while, then the system should indicate this by reusing the same percept identifier. The so-called object permanence phenomenon certainly is a desired property of the environment where the cognitive robot performs object recognition and symbol anchoring in, but it is not required by 
our approach. ${ }^{6}$

Our symbol anchoring approach does not pose further assumptions about the performance of the tracking system besides those mentioned in Section 3.11, i. e., our approach ought to accomplish symbol anchoring appropriately regardless of the performance of the tracking system in use. Moreover, two further symbol anchoring functionalities shall enable our approach to cope with any situation that the recognition system has failed to handle or was not designed for. The specific structure of the symbol anchoring Axiom (4.15) combining the three functionalities in predicate $C C$ facilitates the desired object identification. The purpose of the next functionality called "Find" is twofold, depending on whether the considered object has been anchored previously or not:

1. Those objects that have not yet been associated with a percept hold an empty perceptual estimate inside its anchor fluent (cf. Formula (4.11)). The task of functionality Find in this case is to determine which object symbols and which anchors a given percept fits to.

2. After the tracking of a certain object of the robot's environment has failed, the recognition and tracking system may perceive the same object later again. If the tracking system resumes tracking, it reuses the same percept identifier and the functionality Track applies. In case the tracking system cannot recognize a new percept to correspond to a previous one without doubt, a new percept identifier is used and the functionality Find applies instead. In this case a non-empty, best perceptual estimate is available in the anchor, which guides to find the correct correspondence.

Our approach defines the functionality Find in form of macro $\Delta_{F}$ as follows:

$\Delta_{F}: \mathcal{X} \cup$ PERCID $\times$ OBJCAT $\times$ PERCSIG $\times$ PERCID $\cup\{\perp\} \times$ PERCID $\times$ PERCSIG $\times$ SIT $\times$ STATE

$$
\begin{aligned}
& \Delta_{F}\left(x, o, \Gamma, \pi, \pi_{P}, \Gamma^{\prime}, s, z\right) \stackrel{\text { def }}{=} \\
& \quad(\exists)\left(P\left(\pi_{P}, o, \Gamma_{P}, s\right) \wedge \operatorname{Holds}(A(x, o, \Gamma, \pi), z) \wedge \neg\left(\exists \Gamma_{1}\right)\left(P\left(\pi, o, \Gamma_{1}, s\right)\right) \wedge\right. \\
& \left.\quad \operatorname{Match}\left(x, \Gamma_{P}, z\right) \wedge \operatorname{UpdatePS}\left(\Gamma, \Gamma_{P}, \Gamma^{\prime}\right) \wedge \neg\left(\exists y, \Gamma_{y}\right)\left(\operatorname{Holds}\left(A\left(y, o, \Gamma_{y}, \pi_{P}\right), z\right)\right)\right)
\end{aligned}
$$

For the functionality Find, i. e. macro $\Delta_{F}$, to be applicable, several conditions have to hold simultaneously according to Formula (4.23):

1. The given percept identifier $\pi_{P}$ and the perceptual signature $\Gamma_{P}$ have to pertain to a current percept. Since the symbol anchoring Axiom (4.15) explicitly enumerates all current percepts and asks predicate $C C$ to associate a symbol with each of these percepts, this condition is guaranteed to hold inside $C C$. The percept predicate for percept $\pi_{P}$ is included in the definition of Find for the sake of readability and modularity.

2. Given the existential quantification of variables $x, \Gamma$ and $\pi$ in predicate $C C$, the Formula (4.23) requires that there exists some object anchor in hypothesis $z$ such that further conditions hold.

3. The conjunction of the negated percept predicate for percept $\pi$ and of the anchor of object $x$ means that Find only allows to establish a new correspondence between percept $\pi_{P}$ and object symbol $x$ if no other percept is already associated with object $x$. This applies only in case there was no corresponding percept for object $x$, i. e. $\pi=\perp$ in Formula (4.23), or otherwise if the registered percept has not been perceived in situation $s$.

4. The perceptual and symbolic information about object $x$ have to comply with the perceptual signature $\Gamma_{P}$. For this purpose predicate Match is employed.

\footnotetext{
${ }^{6}$ The object permanence property of environments stipulates that "objects do not cease to exist or change their identity when obscured." [Witkowski et al., 2001, p. 8]
} 
5. The predicate UpdatePS is expected to update the perceptual estimate $\Gamma$ inside the anchor of object $x$ given the new percept $\Gamma_{P}$.

6. Only those percepts $\pi_{P}$ may become associated with object $x$ that do not already correspond with any object, i. e., there must not be an anchor fluent in the considered hypothesis $z$ that bears the percept identifier $\pi_{P}$.

Note that functionality Find is applicable to any object $x$ that satisfies all above-mentioned conditions giving rise to multiple potential correspondences with the considered percept $\pi_{P}$ in general. Also note that both Find and Track are not applicable to the same percept or the same object symbol, although the predicate $C C$ combining all three functionalities does allow this case in the first place. This is due to the following observation.

Observation 4.1 The functionalities Track and Find never apply for the same percept identifier and object identifier considering a certain situation and hypothesis, i.e.

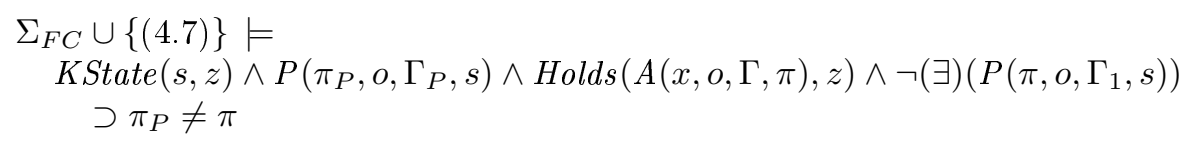

Proof Assume to the contrary that $\pi_{P}=\pi$ is the consequent of (4.24). Then according to the antecedent of $(4.24)$ the conjunct $(\exists)\left(P\left(\pi, o, \Gamma_{1}, s\right)\right)$ would hold true, which contradicts the antecedent. Thus (4.24) is valid.

The functionality Find employs the predicate UpdatePS for the purpose of updating the perceptual estimate of the associated anchor with the perceptual signature of the new percept. Being a kind of sensor fusion technique, the new perceptual estimate is computed by combining the two inputs and selecting the smallest range of perceptual values for each static attribute, and taking on the measurements of dynamic attributes.

$$
\text { UpdatePS : OBJCAT } \times \text { PERCSIG } \times \text { PERCSIG } \times \text { PERCSIG }
$$

$$
\begin{aligned}
& \text { UpdatePS }\left(o, \Gamma_{A}, \Gamma_{P}, \Gamma^{\prime}\right) \equiv \\
& \qquad \begin{array}{l}
\left(a,\left(b^{\prime}, t^{\prime}\right)\right) \in \Gamma^{\prime} \equiv \\
\left(\text { AttrTypeStatic }(o, a) \wedge\left(\gamma \in\left(\Gamma_{A} \cup \Gamma_{P}\right) \wedge \gamma=(a,(b, t)) \supset b \leq b^{\prime} \wedge t \geq t^{\prime}\right) \wedge\right. \\
(\exists \gamma, b, t)\left(\gamma=(a,(b, t)) \wedge b=b^{\prime} \wedge \gamma \in\left(\Gamma_{A} \cup \Gamma_{P}\right)\right) \wedge \\
\left.(\exists \gamma, b, t)\left(\gamma=(a,(b, t)) \wedge t=t^{\prime} \wedge \gamma \in\left(\Gamma_{A} \cup \Gamma_{P}\right)\right)\right) \\
\left.\vee \neg \text { AttrTypeStatic }(o, a) \wedge(\exists \gamma)\left(\gamma=\left(a,\left(b^{\prime}, t^{\prime}\right)\right) \wedge \gamma \in \Gamma_{P}\right)\right)
\end{array}
\end{aligned}
$$

The predicate UpdatePS defines the new perceptual estimate $\Gamma^{\prime}$ to comprise all those tuples $\left(a,\left(b^{\prime}, t^{\prime}\right)\right)$ of perceptual attributes $a$ and ranges of perceptual values $\left(b^{\prime}, t^{\prime}\right)$ such that one of the following conditions holds.

1. In the case of a static perceptual attribute, the current perceptual signature $\Gamma_{P}$ and the previous perceptual estimate $\Gamma_{A}$ are fused by taking the minimal intersecting interval. Nevertheless, the new estimate must be grounded on factual information, i.e., the perceptual uncertainty interval can only be reduced to the extent which is actually sustained by the current percept or any previous percept. The predicate UpdatePS implements this in accordance with our concept of representing perceptual uncertainty described in Section 3.5.

2. For dynamic perceptual attributes the new perceptual estimate simply takes the values of the current percept and disregards any previous values that possibly have been recorded in 
the anchor before. This way predicate UpdatePS also contributes to the consistency of the perceptual estimate with respect to the requirement that the estimate of the anchor includes at most a single perceptual interval (cf. Section 4.2.7).

The definition of the predicate UpdatePS takes the specific meaning of the perceptual signature of percepts and of the best perceptual estimate of anchor fluents into account with respect to the representation of perceptual uncertainty as defined in Sections 4.1.1 and 4.1.2. The simple information fusion implemented in predicate UpdatePS maximizes the information gain for static perceptual attributes. The distinction between static and dynamic perceptual attributes is crucial for the sound computation of our approach in realistic, dynamic environments of cognitive robots. Otherwise the representation of perceptual uncertainty could not be utilized for determining appropriate correspondences between percepts and object symbols to the extent accomplished in our approach.

In summary, the macro $\Delta_{F}$ implements the symbol anchoring functionalities to establish a previously non-existent correspondence and to reacquire a temporarily lost correspondence between a percept and the appropriate object symbol. Thus, our approach fulfils the two remaining important requirements of any approach to the symbol anchoring problem stated by Coradeschi and Saffiotti [2002] and listed in Section 2.3.4.

In comparison with the approaches of Coradeschi and Saffiotti [2001] and of Chella et al. [2004] including their descendant approaches, and in addition to what has been pointed out already above, most obviously they use two separate symbol anchoring functionalities "Find" and "Reacquire" for what our approach accomplishes in the single functionality "Find". This is due to the fact that our approach does not perform prediction of the perceptual signature of the object in question. Since no perceptual information is available in case of their functionality "Find", they have to distinguish between two separate functionalities (cf. Section 2.3.5).

However, the more important difference ought to be seen in the following, which we briefly repeat here. While our approach performs symbol anchoring for all percepts at once in a bottomup manner, i. e., the task is to determine appropriate correspondences with object symbols given a percept, their approach anchors a single object symbol to possibly multiple matching, current percepts in a top-down fashion. We claim that anchoring all current percepts in parallel prepares the cognitive robot to handle ambiguous situations better (cf. Section 4.2). Accordingly, the result is very different: While the approaches of Coradeschi and Saffiotti [2001], Broxvall et al. [2005], Chella et al. [2004] and their descendant approaches yield possibly multiple correspondences for the object symbol in question, our approach determines the complete space of all possible hypotheses of correspondences regarding all known objects automatically.

The details of the implementation of the functions "Update", "Verify" and "Predict" are missing in [Coradeschi and Saffiotti, 2001] unfortunately, but their use in the procedure "Reacquire" suggests that the update of a perceptual signature intermingles symbolic and perceptual information. This would contradict our fundamental concept of independence of symbolic and perceptual information stated in Section 3.1.

\subsubsection{Introducing a New Object}

Realistic application domains typically involve dynamic objects, changing environmental conditions and are at least partly unknown to the cognitive robot. Apparently, the ability of the robot to adapt its behaviour and world model to changing conditions plays a crucial role herein. This concerns especially the symbol anchoring of objects. We have explained above how the functionalities Track and Find adapt the world model of correspondences between object symbols and objects of the world upon acquiring new percepts in our approach. Both functionalities regard individual objects that are already known on the deliberative level. Thus, an additional functionality is necessary - called "New"- that introduces new individual objects, which were unknown to the robot so far, in order to construct a more complete world model concerning objects of interest. Note that our approach does not take unknown, new categories of objects into account.

According to our assumptions about the object recognition and tracking system, our approach 
expects the tracking system to perform reliably and sound, i. e., it shall only track an object as long as this can be performed without doubt about whether the system is still tracking the very same object of the world (cf. Section 3.11). Otherwise, if the certainty of whether it is still tracking the same object dropped due to an obstruction for instance, it shall simply report that there is another object of interest. We distinguish this situation, where a new, fresh percept identifier is assigned to the percept, as follows.

1. The physical object may actually be a completely new object, meaning that the object really has never been recognized by the robot before and there is no individual object symbol referring to it on the deliberative level. The functionality New applies in this case and introduces the percept as a new individual object to the symbol anchoring representation in particular and to the deliberative level in general for future actions with it and for reasoning about it. We will define this functionality below.

2. But the percept may also describe an object of the world which the robot already knows under a different name, which the recognition system failed to associate with a previous percept doubtlessly. The symbol anchoring functionality Find applies in this case, which determines a potential correspondence based on the object's perceived, phenomenal characteristics. The reader is referred to Section 4.2.3 explaining it in detail.

This distinction stresses an important, difficult aspect of symbol anchoring: The fact that the recognition and tracking system reports a percept as being a new one potentially gives rise to both possible cases mentioned above, while the symbol anchoring system cannot determine definitely whether the new percept corresponds to a new physical object or to a known object. The concept of our approach is to take all potential hypotheses into account, to utilize every single evidence for one and against the other hypothesis and to maintain all hypotheses in parallel, until some evidence possibly discloses a contradiction.

Hence, in the case of anchoring a percept, which bears a fresh identifier, the functionality Track does not apply, the functionality Find possibly applies if there are suitable anchors, and the functionality New definitely applies. Our approach defines New in terms of the macro $\Delta_{N}$ like this:

$$
\Delta_{N}: \text { PERCID } \times \text { OBJCAT } \times \text { PERCID } \times \text { PERCID } \times \text { SIT } \times \text { STATE }
$$

$$
\begin{aligned}
& \Delta_{N}\left(x, o, \pi_{P}, n, s, z\right) \stackrel{\text { def }}{=} \\
& \quad\left(\exists \Gamma_{P}\right)\left(P\left(\pi_{P}, o, \Gamma_{P}, s\right) \wedge \neg(\exists y, \Gamma)\left(\operatorname{Holds}\left(A\left(y, o, \Gamma, \pi_{P}\right), z\right)\right) \wedge \operatorname{NewSymbol}(n, x, z)\right)
\end{aligned}
$$

The macro $\Delta_{N}$ applies if the considered percept is a current, valid percept and if it is not already associated with an object symbol via an anchor, because only one symbol is allowed to correspond to a certain percept within the same hypothesis $z$. The macro NewSymbol determines a new, fresh object symbol as follows.

$$
\begin{gathered}
\text { NewSymbol }: \text { PERCID } \times \text { PERCID } \times \text { STATE } \\
\text { NewSymbol }(n, x, z) \stackrel{\text { def }}{=}(\exists t)(\text { Holds }(\text { Offset }(t), z) \wedge x=t+n) \\
\text { Offset }: \text { PERCID } \mapsto \text { FLUENT } \\
\text { Knows }\left(\text { Offset }(0), S_{0}\right)
\end{gathered}
$$

The technique of determining a new, fresh object symbol, which is unique and which complies with the constraints of predicate Consistent in the symbol anchoring Axiom (4.15), employs the 
macro NewSymbol and the fluent Offset. The new fluent Offset is initialized with the value zero in the initial situation $S_{0}$ according to Formula (4.31), i. e., certainly before a new object identifier may be required at all.

The fluent Offset comprises an auxiliary counter which is increased with each application of the symbol anchoring Axiom (4.15) and to be left unchanged otherwise. The Formula (4.31) initializes the fluent Offset equally with the value zero in all possible hypotheses $z$ of situation $S_{0}$. In order to gain uniqueness of new percept identifiers, it is important that the fluent Offset carries the same value in all possible hypotheses $z$ associated with a certain situation $s$, which is achieved using the macro Knows. The symbol anchoring Axiom (4.15) interchanges Offset $(t)$ with Offset $(t+k)$ for each successor state $z^{\prime}$. The function $N o P$ determines the number of percepts available in a given situation $s$, which is the same for each hypothesis $z$ associated with $s$ via $K S t a t e(s, z)$ and which is unique according to the Formula (4.7). Of course, the symbol anchoring action AnchorU, like any other action, can only be applied once in the same situation. Given the initialization of fluent Offset in Formula (4.31) and its uniform update in Axiom (4.15) for all hypotheses of the considered situation, we have shown that the value of fluent Offset will always remain uniform among all hypotheses of any successive situation.

The symbol anchoring Axiom (4.15) increases the value of the fluent Offset precisely by the number of percepts available in the considered situation. Hence, in any situation the fluent Offset bears the maximal natural number that has possibly been used as an identifier so far. The Formula (4.27) defining the functionality New and the Formula (4.29) defining the macro NewSymbol make sure, that values of the range 0..t with Holds (Offset $(t), z)$ are not used twice for identifiers within the same hypothesis, because NewSymbol always adds to the value of fluent Offset. The number $n$ which macro NewSymbol adds to the offset is unique in each situation, because the schematic Axiom (4.15) uses each number of the range $0 . . k$ with $N o P(o, s)=k$ exactly once as a parameter of predicate $C C$, which in turn hands it to macro NewSymbol by means of macro $\Delta_{N}$. Thus, not all values in the range $0 . .(t+k)$ may actually be used, but this way our approach ensures that each value is used at most once for an identifier for the purpose of uniqueness of object symbols. The sort PERCID provides an unlimited supply of fresh identifiers anyway. Moreover, the definition of PERCID as a separate sort to be used solely for new object and percept identifiers together with the requirement $\mathcal{X} \cap$ PERCID $=\emptyset$, Formula (4.9), guarantee consistency and uniqueness of object symbols in our approach.

Note that the functionality New is applicable in all cases where Find is applicable, because the macro NewSymbol inside the definition of macro $\Delta_{N}$ is always satisfiable and both Find and New share the same condition $\neg(\exists)\left(\operatorname{Holds}\left(A\left(y, o, \Gamma, \pi_{P}\right), z\right)\right)$. In the case where multiple functionalities apply, the definition of predicate $C C$ guarantees that the assignments to the fluents $f^{-}$and $f^{+}$ are not mixed up among those functionalities within the same hypothesis, and that each applicable functionality gives rise to its own, separate potential hypothesis, i.e., an applicable functionality is never neglected.

In summary, we have defined the symbol anchoring functionality New, which introduces a new anchor with a new object symbol for an unknown object of the robot's environment automatically. We have shown that the functionality New preserves the uniqueness of percept identifiers and of object symbols. The functionality New complies with our concept of representing and maintaining all possible hypotheses of correspondences in parallel. This functionality is a crucial component of an approach to the symbol anchoring problem in order to enable a cognitive robot to experience yet unknown objects of its dynamic environment.

In comparison with the other approaches summarized in Section 2.3.5 it is not clear how they handle to learn about unknown objects of the environment from the information given in their respective publications. The main difference regarding this task between our approach and those of Coradeschi and Saffiotti [2001, 2002], Broxvall et al. [2005], Chella et al. [2004] and their later improved versions is the direction and extent of anchoring. We have pointed out above that our approach anchors all available percepts to appropriate object symbols in a bottom-up manner. The main objectives for this concept are first, not to neglect any potential hypothesis of correspondences and second, to ground as many correspondences as possible in order to avoid forthcoming ambiguous situations to the largest possible extent. However, the other above-mentioned ap- 
proaches anchor a certain object symbol to appropriate percepts in a top-down manner. Due to limited detail it is not clear how these approaches take the automatic learning of unknown objects into account.

Let's combine all the above-mentioned formulas of our uni-modal symbol anchoring approach and denote them with $\Sigma_{u m s a}$. We call $\Sigma_{u m s a}$ the uni-modal symbol anchoring axioms:

$$
\begin{aligned}
\Sigma_{u m s a} \stackrel{\text { def }}{=} & \{(4.3),(4.4),(4.5),(4.6),(4.7),(4.9),(4.10),(4.11)\} \\
& \cup\{(4.13),(4.15),(4.17),(4.31)\}
\end{aligned}
$$

\subsubsection{Complexity of Hypothesis Space}

This section discusses the complexity of the hypothesis space of correspondences between object symbols and percepts. We have defined a symbol anchoring hypothesis in Section 3.9. Furthermore, we have explained in the Sections 3.10 and 4.1.3 how multiple hypotheses can be represented.

Since the complexity of the hypothesis space depends very much on the application domain, the environmental conditions and the robot's perceptual capacity, only a general discussion is possible. What are the effects of a single application of the symbol anchoring Axiom (4.15) on the hypothesis space? Obviously, if there are no percepts available, this axiom reduces to Formula (4.16), which infers no changes at all to the successor knowledge state. In this case the hypothesis space remains precisely the same.

Otherwise if there is a certain number of percepts available, i.e. $(N o P(s)=k) \wedge(k>0)$, the effects of the symbol anchoring transaction on the hypothesis space vary in a wide range. For instance, each current percept may correspond to a known object that is being tracked, such that functionality Track applies to each of the $k$ percepts. In this case the application of the symbol anchoring axiom for action Anchor $U$ produces no additional hypotheses nor causes the elimination of current hypotheses. Hence the extent of the hypothesis space remains constant and the symbol anchoring Axiom (4.15) only updates the representation of all the anchors involved.

A closer look at the definition of our functionality Track, Formula (4.19), reveals that although in principle predicate $C C$ allows to spawn further, alternative correspondences with the considered percept due to the disjunction of the three functionalities, Track precludes the applicability of Find and New for the same percept and the same hypothesis. Hence, if functionality Track is applicable to a certain percept, the existing correspondence becomes the only correspondence for this percept in the successor hypothesis space for the considered original hypothesis and situation. The interface definition of our symbol anchoring approach with the object recognition system motivates this concept: According to our assumptions stated in Section 3.11, the tracking system guarantees that measuring and reporting a series of percepts bearing the same identifier in successive situations indicates that this series of percepts originates from the same physical object. Our symbol anchoring functionality Track utilizes this assumption on the tracking system in terms of a single, possible, successive correspondence if applicable.

Next we are going to discuss the functionality Find, defined in Formula (4.23), and functionality New, defined in Formula (4.27), with respect to their effects on the complexity of the hypothesis space. Recall that Find is only applicable if Track is not. Considering a certain, current percept and a certain hypothesis, and given that this percept is not registered in an anchor already, the application of functionality Find determines the complete set of all potential object symbols $(m$ in number), whose symbolic and perceptual information fit the given percept, and transforms the considered hypothesis into $m$ potential successive hypotheses-one hypothesis for each correspondence with one of the matching, known object symbols. Furthermore, the functionality New applies in the case and situation considered and adds an additional hypothesis to the successor situation wherein the percept corresponds to a new object symbol. The number $m$ of new correspondences established by Find depends on the number of known objects that have very similar characteristics compared with the new percept. Most notably, Find may not be applicable at all, i.e. $m=0$, such that only functionality New anchors the considered percept with a new object symbol. In the latter case, the number of hypotheses stays the same. 
Basically, the above explanations suggested that the functionalities employed in our approach cause the hypothesis space to enlarge always or at least to keep its size, which is actually not the case. Surely it would be very desirable to keep the number of potential, alternative hypotheses as small as possible in order to facilitate an effective and precise world model, which is crucial for cognitive robots operating in the real world. Indeed, our symbol anchoring approach implements a means for eliminating hypotheses that contradict with sensory data, which we will explicate below.

Our approach as well as other typical cognitive robotic systems are based on the cognitive theory called phenomenalism (cf. Section 2.1.2). In this view, perceiving phenomenal features of objects designates the most important means for synchronizing the internal world model with the real world. Our idea is based on the assumptions about the robot's perception of the environment described in Section 3.1, most importantly the assumption of disregarding perceptual seemings, and on the basic concept of knowledge update inherited from the Fluent Calculus (cf. Section 2.4). Recalling the latter, a knowledge update axiom specifies that for any successor state to exist, such a successor state can only be derived from an existing original state and that certain conditions have to hold including the consistency with sensor data (cf. Formula (2.5)). More precisely, a knowledge update axiom specifies exactly those and only those transactions that lead to consistent successive hypotheses, and which comply with the latest perceptual findings. Those states which no transaction applies to will be disregarded automatically and will not generate a single successor state. Our symbol anchoring axiom, Formula (4.15), inherits this principle. This axiom defines exactly those conditions and transactions that allow it to generate consistent successor hypotheses of correspondences. The conditions defined therein for a successor state to exist include the conjunction of individual percept predicates ( $k$ in number), a conjunction of individual predicates $C C$ for each percept, and the predicate Consistent, while the remaining conditions are readily satisfiable. Due to Formula (4.7), the conjunction of percept predicates is valid by assumption.

The conjunction of predicates $C C$ in the Axiom (4.15) in the way explained above plays the crucial role in determining the hypothesis space. Below we will provide a proof showing that the state transitions determined by the symbol anchoring Axiom (4.15) lead from consistent states to consistent successor states only (cf. Section 5.6.1). We will also show that the hypothesis space computed by our approach never collapses (cf. Section 4.2.6).

\subsubsection{Eliminating Inconsistent Hypotheses}

Next, we are going to point out the kind of hypotheses that the symbol anchoring Axiom (4.15) disregards automatically due to an inconsistency. The prerequisites of our functionalities Track, Find and New can be partitioned into those where an anchor fluent corresponds directly to the considered percept, and those where there is no corresponding anchor fluent. In the latter case functionality New readily applies without further restrictions. In the former case only the functionality Track applies if the anchor matches the considered percept with respect to predicate Match and if the new perceptual signature can be fused with the anchor's perceptual estimate using the predicate UpdatePS. Let "contradiction" denote the specific former case where these additional prerequisites are not fulfilled. The following formula specifies this case of a contradiction

$$
(\exists)\left(\operatorname{Holds}(A(x, o, \Gamma, \pi), z) \wedge P\left(\pi, o, \Gamma_{P}, s\right) \wedge \neg(\exists)\left(\operatorname{Match}\left(x, \Gamma_{P}, z\right) \wedge \operatorname{UpdateP} S\left(\Gamma, \Gamma_{P}, \Gamma^{\prime}\right)\right)\right)
$$

for a certain situation $s$, hypothesis $z$ and a certain percept $\pi$. In contrast to the functionality Track, the case of a contradiction refers to situations wherein the predicate Match or UpdatePS fails. Each potential hypothesis where a contradiction arises from the considered variable substitution will be neglected automatically. In other words, a hypothesis that has been tracked and updated successfully so far becomes extinct upon acquiring contradicting new perceptual information for instance. Such hypotheses simply die out.

The two potential principles that cause a contradicting hypothesis to become extinct are incompleteness and imprecision of perceptual and symbolic information. An incomplete symbolic 


\begin{tabular}{|c|c|c|}
\hline anchor for object $\mathrm{x} \backslash$ percept $\pi_{c}$ & $\neg P\left(\pi_{c}, o, \Gamma_{c}, s\right)$ & $P\left(\pi_{c}, o, \Gamma_{c}, s\right)$ \\
\hline \hline$\left(x, \pi_{c}\right)$ & case 0: \% & case 1: ideal \\
\hline$(x, \perp)$ & case 2: not grounded & case 3: wrong \\
\hline$(x, \pi) \wedge \pi \neq \pi_{c}$ & case 4: wrong & case 5: wrong \\
\hline
\end{tabular}

Table 4.1: Partition of the hypothesis space with respect to the correct vs. alternative hypotheses.

specification of an object gives rise to multiple matching percepts in the first place, but the multitude of these alternative hypotheses shall die out when a more specific symbolic description becomes known. For instance, in the extreme case where the symbolic description of a certain object is completely empty, the functionality Find would propose an alternative correspondence for each percept of the same kind of object, such that virtually every percept of the requested kind matches the object to be anchored. Furthermore, if the perceptual signature and the perceptual estimate contain only sparse information about the physical object, the perceived object has to be considered as a potential candidate for too many known objects. This is an undesirable situation because it hinders the cognitive robot from effective, goal-directed planning, reasoning and acting. In general, the more distinctive the perceptual and symbolic signatures of an object on the symbolic level and of a percept are, the fewer potential candidates give rise to alternative hypotheses. Furthermore, a percept is the more distinctive the more precise its perceptual information is, the more perceptual attributes are measured and the fewer similar objects exist in the world.

The above-mentioned mechanism invalidates and eliminates inconsistent hypotheses from the hypothesis space. However, it is important to make sure that at least one hypothesis remains. Obviously, we would expect the correct one to be among the remaining hypotheses. In order to show that our approach complies with these prerequisites, the Table 4.1 lists all possible cases of correspondences that the application of the symbol-anchoring Axiom (4.15) may compute.

Lemma 4.2 The correct hypothesis of correspondences between object symbols and percepts is guaranteed to be among those generated by the symbol-anchoring Axiom (4.15) in the successor situation.

Lemma 4.3 A hypothesis of correspondences that proved to be actually wrong may be retracted from the hypothesis space safely, because the symbol-anchoring Axiom (4.15) guarantees that at least another one remains existent in the hypothesis space, i. e., the hypothesis space cannot become void.

Proof In the breakdown of Table 4.1 the object $x$ denotes the object symbol which is considered for anchoring, and $\pi_{c}$ denotes the percept of the physical object which symbol $x$ actually refers to, i. e., in case the correspondence is correct. ${ }^{7}$ The columns of this table distinguish between the two cases of whether or not the correct percept is available, i. e., whether $P\left(\pi_{c}, o, \Gamma_{c}, s\right)$ or $\neg P\left(\pi_{c}, o, \Gamma_{c}, s\right)$ holds, respectively. The first line of Table 4.1 refers to the successor hypothesis where the correct correspondence between symbol $x$ and percept $\pi_{c}$ has been established successfully. The specific case number 0 in the table, where percept $\pi_{c}$ is actually not available, simply cannot occur because it is logically inconsistent. Conversely, case number 1 represents the ideal case wherein the physical object has been perceived and the correct correspondence with its percept has been determined. Given our assumptions about the robot's perception of the environment described in Sections 3.1 and 3.11, the functionality Find is applicable in this case and guarantees to yield the desired correspondence between object $x$ and percept $\pi_{c}$ (cf. Section 4.2.3). Moreover, given these assumptions the hypothesis of the correct correspondence will not be invalidated, because this can only be triggered by the existence of inconsistent information. Hence, if some hypothesis becomes inconsistent and is eliminated by the symbol anchoring axiom, the hypothesis of the correct correspondence remains existent if the object was perceived (case

\footnotetext{
${ }^{7}$ The subscript $C$ indicates the correct hypothesis from an omniscient perspective.
} 
number 1). The other case where the object was not perceived will be discussed below for case numbers 2 and 4 .

The case number 2 of Table 4.1 represents the situation where the object symbol $x$ is not anchored to any current percept and the physical object, which $x$ stands for, was not perceived. Although object $x$ is not grounded in this situation, we cannot do any better than that. This hypothesis correctly represents the actual situation. Since this hypothesis is consistent, it cannot be invalidated and eliminated.

The symbol anchoring Axiom (4.15) also determines an alternative hypothesis, where the actual percept is associated with a different object symbol than the correct object $x$, while $x$ is not grounded. This case denoted as case number 3 apparently represents a wrong, alternative hypothesis for percept $\pi_{c}$ and object symbol $x$. However, given the assumptions of Sections 3.1 and 3.11, our approach guarantees to compute the correct hypothesis with the correspondence denoted as case number 1 , too, as described above. Therefore, it is a matter of identifying this hypothesis of case number 3 as being the wrong one, preferring the one of case number 1 , and invalidating it as soon as possible.

The functionality Find may also associate the object symbol $x$ with an appropriate, alternative percept $\pi$ which does not refer to the correct physical object, i.e. $\pi \neq \pi_{c}$. Obviously, this case called number 4 in Table 4.1 represents a wrong, alternative hypothesis. As the functionality New always applies if Track does not apply, New is guaranteed to assign a fresh object symbol to percept $\pi$ in an alternative hypothesis such that object $x$ remains not grounded. The latter case coincides with case number 2. Hence, although the correspondence of case number 4 is wrong, the correct correspondence of case number 2 exists, too. It is the task of a suitable preference criterion to determine the right hypothesis, or the task of the planning system to gain further evidence sustaining the correctness of the alternative hypothesis.

In the case number 5 the object $x$ is associated with the wrong percept, while the correct percept $\pi_{c}$ is available, which turns it into a wrong, alternative hypothesis. However, for reasons explained for the case number 1, the correct hypothesis is guaranteed to exist, too. The above rationale about the possible correspondences resulting from applying our symbol anchoring Axiom (4.15) has shown that:

1. The correct correspondence is guaranteed to be among those determined by the axiom. This shows the Lemma 4.2 .

2. A hypothesis that is actually wrong may be eliminated while at least another one remains existent in the hypothesis space. Because of item 1, at least the correct hypothesis remains existent, such that the hypothesis space cannot collapse. This shows the Lemma 4.3.

This reasoning regarding a single correspondence of one object symbol naturally applies to a combination of correspondences for all object symbols forming a whole hypothesis of correspondences. The above results may be generalized to a whole hypothesis rightly, because individual correspondences are represented as individual anchors, while the predicate Consistent and the symbol anchoring Axioms $\Sigma_{u m s a}$ make sure to respect the mutual dependencies among multiple anchors of a hypothesis.

In summary, imprecision of symbolic and perceptual specifications of an object may cause many alternative hypotheses. For example, the disjunction of several possible perceptual predicates or the exclusion of just a few individual predicates constitute imprecise symbolic specifications of a certain attribute of an object. The imprecision of sensory information about a physical object causes very similar effects. In the case of contradicting information our symbol-anchoring Axiom (4.15) provides an effective means to invalidate such inconsistent world models.

\subsubsection{Consistency of Perceptual Estimates}

The predicate UpdatePS presupposes that the perceptual estimate inside an anchor fluent includes at most one interval of values for each perceptual attribute (cf. Formula (4.25)). Otherwise, especially in the case of multiple disjoint intervals of values of the same attribute, predicate UpdatePS 
would affect the whole computation involving inconsistent results due to invalid intervals $(b, t)$ with $b>t$. The predicate Match also presupposes appropriate perceptual estimates for comparing them with the signature of a percept based on the intersection of intervals. We have posed this requirement already regarding percepts in Formula (4.5).

Next, we are going to show that an additional domain constraint for the purpose of implementing the requirement of uni-modal distributions of attribute values of perceptual estimates in anchor fluents is not necessary.

Lemma 4.4 The symbol anchoring Axioms $\Sigma_{\text {umsa }}$ ensure the uni-modality of perceptual attributes inside the perceptual estimate of anchor fluents, i. e.:

$$
\begin{gathered}
\Sigma_{\text {umsa }} \cup \Sigma_{F C}=(\forall)\left(\operatorname{KState}(s, z) \wedge \operatorname{Holds}(A(x, o, \Gamma, \pi), z) \wedge \gamma_{1} \in \Gamma \wedge \gamma_{2} \in \Gamma \wedge\right. \\
\left.\gamma_{1}=\left(a, i_{1}\right) \wedge \gamma_{2}=\left(a, i_{2}\right) \supset i_{1}=i_{2}\right)
\end{gathered}
$$

Proof The proof is based on the idea that given a consistent initial situation and a consistent update from one situation to the next, all successor situations will be consistent, too.

Induction base: The domain constraint of Formula (4.11) requires that each perceptual estimate is empty for all anchor fluents existing initially. Hence, the assumption of Formula (4.34) is satisfied for the initial situation $S_{0}$ of course.

Induction hypothesis: Assume a situation $s$ which the above Formula (4.34) holds for.

Induction step: Only predicate UpdatePS manipulates the perceptual estimate inside anchor fluents. Perceptual attributes are known to be of either static or dynamic kind (cf. Section 3.6). In case of a dynamic attribute the new perceptual estimate equals the corresponding component of the perceptual signature $\Gamma_{P}$ by definition of predicate UpdatePS (cf. Formula (4.25)). Furthermore, the new perceptual estimate $\Gamma^{\prime}$ deduced by UpdatePS will include at most one interval of values for a dynamic attribute thanks to the constraint on uni-modal perceptual attributes of percepts (cf. Formula (4.5)).

Depending on the number $n$ of value intervals available in the considered perceptual estimate and the perceptual signature, i.e. among $\left(\Gamma_{A} \cup \Gamma_{P}\right)$, for a certain static perceptual attribute considered by predicate UpdatePS, we distinguish the following cases. For $n=0$ the predicate UpdatePS determines that the new estimate $\Gamma^{\prime}$ will not contain any value interval for the considered attribute. Obviously, the Assumption (4.34) holds in this case. Regarding $n=1$ the predicate UpdatePS defines the resulting perceptual estimate as $\left(a,\left(b^{\prime}, t^{\prime}\right) \in \Gamma^{\prime}\right.$ with $\left(b^{\prime}, t^{\prime}\right)=(b, t)$, i.e., the new estimate of the considered attribute simply takes on the given perceptual interval. Hence, (4.34) holds in accordance with the domain Constraint (4.4). In case $n>1$, given the Assumption (4.5) about uni-modal perceptual attributes regarding percepts, and provided that the uni-modal attributes are recorded in the considered, original hypothesis in accordance with the idea of this proof, which we pointed out above to hold for the initial situation, then $n=2$. Since predicate UpdatePS is employed in combination with predicate Match in the functionalities Track and Find only, and since Match ensures that the two perceptual intervals intersect for each static perceptual attribute, the resulting perceptual interval $\left(b^{\prime}, t^{\prime}\right)$ determined by UpdatePS is consistent and equals the intersection of the one of the perceptual estimate inside the considered anchor and the one of the considered percept.

This way, the consistency of the perceptual estimate is preserved by predicate UpdatePS. An additional domain constraint similar to (4.34) is not necessary as has been shown above and because UpdatePS is the only predicate manipulating the perceptual estimate inside anchor fluents.

\subsubsection{Groundedness of Correspondences}

Recall the use and purpose of symbol anchoring as being a method for establishing and for maintaining the correspondences between symbols and percepts, both of them referring to the same 
object in the environment of a cognitive robot. Our approach represents correspondences in form of anchor fluents which are maintained by a specific knowledge update axiom for symbol anchoring. The point of time of performing such an update is determined solely by the programmer of the application. Obviously, it makes much sense to perform the symbol anchoring update only if there are some current percepts available. Only in this case our symbol anchoring approach may utilize some evidence for or against certain hypotheses of correspondences, or enables the robot to experience new objects of the world. This shows that keeping all correspondences up-to-date tends to be quite hard for a symbol anchoring system in realistic, dynamic application domains, a problem that is related to the so-called representation problem [Wooldridge and Jennings, 1995]. ${ }^{8}$

The above-mentioned difficulties complicate an appropriate implementation of the most crucial and desired property of symbol anchoring systems called the groundedness of object symbols. The groundedness of object symbols denotes the degree or extent to which the object symbols refer to the correct, real-world objects, or in other words, how "tight" the symbolic representation matches the reality via anchoring object symbols to percepts. ${ }^{9}$

For example, the symbol anchoring system is typically asked to provide grounded correspondences for those objects that the cognitive robot is going to manipulate next. The difficult question is, how the symbol anchoring system could determine the groundedness of certain object symbols appropriately and notify other systems about it, like the planning and reasoning system. The following causes contribute to the representation problem of our approach in particular. The representation of correspondences in terms of anchor fluents remains unchanged between successive symbol anchoring transactions, and the time passed in between may differ significantly. Thus, an object symbol being grounded through an anchor fluent may actually be no longer grounded if the dynamics of the physical object have changed since this object was anchored most recently. Moreover, the most recent anchoring transaction of an individual, grounded object symbol may have been performed several symbol anchoring transactions ago, because each symbol anchoring transaction focuses its attention on the particular object category under consideration in our approach. ${ }^{10}$

Among the causes influencing the groundedness of correspondences between object symbols and physical objects are the robot's own actions, in particular movement actions, because typically those actions entail a change of the current range of perception. It appears far too difficult and brittle to model each action's effects on the robot's perception for the purpose of anticipating whether or not certain correspondences remain grounded, neglecting issues of computational complexity for a moment. This method would also infringe the modularity and autonomy of our symbol anchoring system with respect to the other system components of the deliberative level. Another approach could be to automatically break all grounded correspondences with percepts immediately upon performing any action. This method infers similar disadvantages as mentioned above.

Furthermore, the dynamic, non-deterministic world itself causes the robot's perception to change, which cannot be anticipated in general, such that the groundedness of object symbols naturally becomes questionable after considerable time has passed. Introducing a time stamp for all correspondences indicating when the most recent anchoring happened would amount to more computational effort and would require heuristics and probabilities for reasoning about correspondences, which is not the focus of our work.

Our approach readily allows to verify the groundedness of a certain object symbol $x$ for a certain hypothesis $z$ by means of the query

$$
(\exists)(\operatorname{KState}(s, z) \wedge \operatorname{Holds}(A(x, o, \Gamma, \pi), z) \wedge \pi \neq \perp)
$$

${ }^{8}$ Briefly, the representation problem addresses how to symbolically represent information about complex realworld entities and processes [Wooldridge and Jennings, 1995].

${ }^{9}$ The meaning of groundedness differs in the fields of research on symbol anchoring and on symbol grounding (cf. Section 2.2.3).

${ }^{10} \mathrm{~A}$ simple method to reduce this problem is to expand the attention to all kinds of objects, such that symbol anchoring regards all perceivable objects in the world at once. However, we argued above for using object categories (cf. Section 3.3). 
Nevertheless, the Formula (4.35) entails the problem that we do not know, how old the percept $\pi$ is, i.e., when the most recent anchoring transaction took place, because this component of the anchor fluent is not reset when the associated percept vanishes. Instead of the above query, we extend our approach with the predicate IsGrounded:

$$
\text { Is Grounded }: \mathcal{X} \cup \operatorname{PERCID} \times \operatorname{SIT} \times \operatorname{STATE}
$$

$$
\begin{aligned}
& \text { IsGrounded }(x, s, z) \equiv \\
& \quad(\exists)\left(\operatorname{KState}(s, z) \wedge \operatorname{Holds}\left(A\left(x, o, \Gamma_{A}, \pi\right), z\right) \wedge P\left(\pi, o, \Gamma_{P}, s\right)\right)
\end{aligned}
$$

In comparison with the Formula (4.35), the new predicate IsGrounded represents the property of a correspondence to be grounded much more precisely, because there are situations and correspondences where (4.35) holds but where no current percept is available. The existential quantification of the variables of the percept predicate is particularly useful, because it allows that tracking may fail and that the percept identifier corresponding with the same object symbol may change. Thus, for an object symbol to be grounded, it is sufficient that some current percept is associated. The symbol anchoring approaches of Coradeschi and Saffiotti [2000, 2001], Coradeschi et al. [2001] implemented basically the same notion of groundedness of an object symbol as we defined it above (cf. Section 2.3.5).

In summary, our concept of groundedness of object symbols and its definition in terms of predicate IsGrounded emphasize that the symbol anchoring transaction shall be coupled with sensing actions. Since our approach does not involve reasoning about and planning of actions on purpose, which would intermingle symbol anchoring with domain-dependent axiomatization, our approach retains a domain-independent, modular solution.

\subsection{Preferences among Alternative Hypotheses}

We have pointed out above that the application of the uni-modal symbol anchoring axiom of Formula (4.15) causes multiple, alternative hypotheses to be generated in general in situations where percepts are available (cf. Section 4.2.5). Recall that the cognitive robot employs symbol anchoring for the purpose of grounding object symbols representing objects of current interest in perceptual measurements for subsequent actions with those objects. Apparently, the existence of a multitude of alternative hypotheses of correspondences of a certain object symbol inhibits the prerequisites for subsequent object manipulations to become true. Nevertheless, the Section 3.10 highlighted that we consider maintaining all potential and consistent hypotheses of correspondences in parallel, because this facilitates handling ambiguous situations. Our approach signifies a particular hypothesis as being the current belief state with respect to symbol anchoring among all hypotheses of correspondences of the considered situation. Given the belief state, the cognitive robot shall not be bothered with the need to reason about alternative hypotheses when it plans its actions and reasons about its knowledge, but can concentrate on the readily available, unique belief state that is determined by the symbol anchoring system automatically. In general a belief state is defined as "a rational idealization of a cognitive state of some individual at a given point of time" in [Gärdenfors, 1988].

It is important to observe that there is no guarantee for the signified world model to be actually the correct one, although all information obtained from perceiving it and interacting with it so far have sustained it consistently. This is due to the fact that the ground truth of the correctness of a correspondence between an object symbol and the physical object it denotes — naturally—cannot be determined by the cognitive robot itself. ${ }^{11}$ Therefore our approach associates a certain situation and a certain hypothesis adaptively with the belief state of this situation.

One may want the belief state to allow to point to multiple hypotheses in case all of those stand equally for the most preferred hypotheses. In contrast, one may want to strictly require the

\footnotetext{
${ }^{11}$ Otherwise, the symbol anchoring problem would not be a problem at all.
} 
preference relation to designate a single hypothesis only to represent the belief state uniquely. To this end our approach includes two different versions of the preference relation that implement these two cases, which we will introduce in the next two sections.

\subsubsection{The Unique Preference Relation}

Determining the most preferred hypothesis of correspondences, i.e. the belief state of the considered situation, is implemented using the predicate BState, which is based on the preference relation represented by the predicate Pref as follows.

BState : SIT $\times$ STATE

Pref $:$ SIT $\times$ STATE $\times$ STATE

DiffCorr : STATE $\times$ STATE

$$
\begin{aligned}
& \text { BState }(s, z) \equiv \\
& \operatorname{KState}(s, z) \wedge(\forall)\left(\operatorname{KState}\left(s, z_{2}\right) \wedge \operatorname{DiffCorr}\left(z, z_{2}\right) \supset \operatorname{Pref}\left(s, z, z_{2}\right)\right) \\
& \operatorname{DiffCorr}\left(z_{1}, z_{2}\right) \equiv \\
& (\exists)\left(\operatorname{Holds}\left(A\left(x, o, \Gamma_{1}, \pi_{1}\right), z_{1}\right) \wedge \operatorname{Holds}\left(A\left(x, o, \Gamma_{2}, \pi_{2}\right), z_{2}\right) \wedge \pi_{1} \neq \pi_{2}\right. \\
& \left.\quad \vee \operatorname{Holds}\left(A\left(x_{1}, o, \Gamma_{1}, \pi\right), z_{1}\right) \wedge \operatorname{Holds}\left(A\left(x_{2}, o, \Gamma_{2}, \pi\right), z_{2}\right) \wedge x_{1} \neq x_{2}\right)
\end{aligned}
$$

According to this definition, the predicate $\operatorname{BState}(s, z)$ designates a particular hypothesis $z$ of situation $s$ as the belief state if the hypothesis $z$ is preferred to all other hypotheses in the considered situation according to the predicate Pref. Predicate BState is designed to take the existence of just a single hypothesis into account.

The predicate DiffCorr determines whether the given two states actually refer to two different hypotheses of correspondences, because states may include arbitrary other fluents or symbolic information, such that a single symbol anchoring hypothesis may be associated with a number of states (cf. Section 3.9). To this end, predicate DiffCorr only compares anchor fluents and neglects all other components that may constitute a state. As you can see in Formula (4.38), predicate DiffCorr defines that two states refer to different hypotheses if the same object symbol corresponds to different percept identifiers or vice versa in the given states. The predicate DiffCorr implicitly requires the existence of at least one anchor fluent in each given state. This condition is important as a hypothesis representation may not contain any fluents at all, which especially may hold in the initial situation.

It is important to recognize the difference between a knowledge state and a belief state in terms of their transaction from one situation to the next. As explained above, the symbol anchoring Axiom (4.15) updates knowledge states and symbol anchoring hypotheses towards the next situation. In contrast, the predicate BState of Formula (4.37) adapts the belief state dynamically in each situation anew.

Unfortunately, it seems to be hardly possible to come up with general, domain-independent, though effective rules for the preference relation among alternative hypotheses, i.e., without resorting to specific features or heuristics that vary with the application domain. In accordance with our objective of devising a domain-independent approach to the symbol anchoring problem, we discuss general characteristics of the preference relation here. We assume a particular preference criteria to be defined by the application engineer as part of the domain axiomatization using Formula $\Phi\left(s, z_{1}, z_{2}\right)$, which is employed by our approach, such that:

$$
\operatorname{Pref}\left(s, z_{1}, z_{2}\right) \equiv \Phi\left(s, z_{1}, z_{2}\right)
$$


The definition of BState requires the preference predicate Pref to establish a strict ordering among all symbol anchoring hypotheses of the given situation. Regarding the predicate $\operatorname{Pref}\left(s, z_{1}, z_{2}\right)$ as a relation between the given hypotheses $z_{1}$ and $z_{2}$ of situation $s$, the external specification of the preference criterion has to agree with the following characteristics.

- The Formula (4.37) defining predicate BState requires the predicate Pref to form an irreflexive relation among symbol anchoring hypotheses:

$$
(\forall)\left(\operatorname{Pref}\left(s, z_{1}, z_{2}\right) \supset \operatorname{KState}\left(s, z_{1}\right) \wedge \operatorname{KState}\left(s, z_{2}\right) \wedge \operatorname{DiffCorr}\left(z_{1}, z_{2}\right)\right)
$$

- For the ordering of hypotheses to be effective, the preference predicate has to implement an asymmetric relation, i.e.

$$
\neg(\exists)\left(\operatorname{Pref}\left(s, z_{1}, z_{2}\right) \wedge \operatorname{Pref}\left(s, z_{2}, z_{1}\right)\right)
$$

- Since each hypothesis representation includes an individual number of anchor fluents, an individual number of anchored correspondences, different degrees of groundedness for each anchor, etc., the preference relation establishes a transitive hierarchy of hypotheses such that:

$$
(\forall)\left(\operatorname{Pref}\left(s, z_{1}, z_{2}\right) \wedge \operatorname{Pref}\left(s, z_{2}, z_{3}\right) \supset \operatorname{Pref}\left(s, z_{1}, z_{3}\right)\right)
$$

- The preference relation is required to be able to order any hypothesis with respect to the belief state. In other words, the preference criteria have to comply with a connex relation among symbol anchoring hypotheses, i. e.

$$
\begin{gathered}
(\forall)\left(K \operatorname{Ktate}\left(s, z_{1}\right) \wedge \operatorname{KState}\left(s, z_{2}\right) \wedge \operatorname{DiffCorr}\left(z_{1}, z_{2}\right)\right. \\
\left.\supset\left(\operatorname{Pref}\left(s, z_{1}, z_{2}\right) \vee \operatorname{Pref}\left(s, z_{2}, z_{1}\right)\right)\right)
\end{gathered}
$$

Altogether, the above criteria form a total order on the set of hypotheses available in a certain situation. The total ordering is also important for our approach to make sure that the second best hypothesis is determined automatically if the previously most preferred hypothesis became invalid due to some logical inconsistency (cf. Section 4.2.6). Hence, while the belief state determines the most preferred hypothesis, the preference relation among all hypotheses computes further, ordered alternatives.

Both irreflexivity and transitivity have been proposed to be necessary logical properties of preferences between perceptibles by Bell and Huang [1999]. The perception hierarchy resulting from their work can be compared with the hierarchy of hypotheses of our approach, since both approaches incorporate new perceptual evidence in accordance with the concept of "seeing is believing" (cf. Section 2.1.2).

\subsubsection{The Set-Based Preference Relation}

The predicates BState and Pref representing the belief state and the preference relation respectively, as defined in Section 4.3.1 above, are not applicable if not every pair of hypotheses is comparable with respect to preferring one to the other. In other words, we need a more flexible definition of the preference relation if the preference criteria could possibly determine the very same level of preference for several different hypotheses. This is typically the case if multiple hypotheses were developed individually in parallel during the course of action, while the structure of their representation is very similar, e.g. using the same object symbols. For this purpose, we define a preference relation on sets of hypotheses based on the predicate PrefSets:

$$
\text { PrefSets }: \mathrm{SIT} \times \mathcal{P}(\mathrm{STATE}) \times \mathcal{P}(\mathrm{STATE})
$$

The predicate $\operatorname{PrefSets}\left(s, \zeta_{1}, \zeta_{2}\right)$ represents that all hypotheses of set $\zeta_{1}$ are preferred to all those of set $\zeta_{2}$ in situation $s$. Similarly to the unique preference relation represented by the predicate 
Pref, our approach assumes the programmer of the application domain to provide a well-defined, specialized preference relation using formula $\Psi\left(s, \zeta_{1}, \zeta_{2}\right)$ such that:

$$
\operatorname{PrefSets}\left(s, \zeta_{1}, \zeta_{2}\right) \equiv \Psi\left(s, \zeta_{1}, \zeta_{2}\right)
$$

The predicate PrefSets has to comply with the following constraints regarding symbol anchoring in order to yield well-defined preference criteria.

$$
\begin{aligned}
& \left(\operatorname{PrefSets}\left(s, \zeta_{1}, \zeta_{2}\right) \supset \zeta_{1} \neq \emptyset\right) \wedge \\
& \left(\operatorname{PrefSets}\left(s, \zeta_{1}, \zeta_{2}\right) \wedge z_{1} \in \zeta_{1} \supset \operatorname{KState}\left(s, z_{1}\right)\right) \wedge \\
& \left(\operatorname{PrefSets}\left(s, \zeta_{1}, \zeta_{2}\right) \wedge z_{2} \in \zeta_{2} \supset \operatorname{KState}\left(s, z_{2}\right)\right)
\end{aligned}
$$

Furthermore, the formula $\Psi$ only forms well-defined preference criteria if the following constraints hold.

- Obviously, a preference can only be represented by an irreflexive relation. Note that this property refers to different hypotheses of correspondences in contrast to individual knowledge states (cf. Formula (4.39)):

$$
\operatorname{PrefSets}\left(s, \zeta_{1}, \zeta_{2}\right) \wedge z_{1} \in \zeta_{1} \wedge z_{2} \in \zeta_{2} \supset \operatorname{DiffCorr}\left(z_{1}, z_{2}\right)
$$

- Being a directional relation, the preference criteria involve asymmetry:

$$
\begin{aligned}
& \operatorname{PrefSets}\left(s, \zeta_{1}, \zeta_{2}\right) \wedge z_{1} \in \zeta_{1} \wedge z_{2} \in \zeta_{2} \\
& \supset \neg\left(\exists \zeta_{3}, \zeta_{4}\right)\left(\operatorname{PrefSets}\left(s, \zeta_{3}, \zeta_{4}\right) \wedge z_{2} \in \zeta_{3} \wedge z_{1} \in \zeta_{4}\right)
\end{aligned}
$$

- A total order among hypotheses requires the predicate PrefSets to implement a transitive relation:

$$
\operatorname{PrefSets}\left(s, \zeta_{1}, \zeta_{2}\right) \wedge \operatorname{PrefSets}\left(s, \zeta_{2}, \zeta_{3}\right) \wedge \zeta_{1} \cap \zeta_{3}=\emptyset \supset \operatorname{PrefSets}\left(s, \zeta_{1}, \zeta_{3}\right)
$$

Based on the predicate PrefSets for ordering sets of hypotheses according to some well-defined preference criteria, we introduce a set-based version of a belief state:

$$
\begin{gathered}
\text { BStateSet }: \text { SIT } \times \mathcal{P}(\text { STATE }) \\
\text { BStateSet }(s, \zeta) \equiv\left(\exists \zeta_{2}\right)\left(\text { PrefSets }\left(s, \zeta, \zeta_{2}\right)\right) \wedge \neg\left(\exists \zeta_{2}\right)\left(\operatorname{PrefSets}\left(s, \zeta_{2}, \zeta\right)\right)
\end{gathered}
$$

The predicate BStateSet $(s, \zeta)$ represents that each symbol anchoring hypothesis $z$ of the set $\zeta$ is considered likewise one of the most preferred hypotheses of the situation $s$. The predicate BStateSet $(s, \zeta)$ only holds for $\zeta$ according to Formula (4.40) if no other set of hypotheses is preferred to $\zeta$. The Formula (4.40) also requires $\left(\exists \zeta_{2}\right)\left(\operatorname{PrefSets}\left(s, \zeta, \zeta_{2}\right)\right)$ to hold for $\zeta$. This is guaranteed even and especially in the case where the hypotheses $\zeta$ are the only ones existing in situation $s$, i.e. with $\zeta_{2}=\emptyset$. Note that the predicate BStateSet also accounts for situations where just a single hypothesis of correspondences exists, e.g. the initial situation.

In comparison, both predicates Pref and PrefSets represent a total ordering among symbol anchoring hypotheses forming an irreflexive, asymmetric and transitive relation. We intentionally omitted PrefSets to define a connex relation in order to allow that the preference criteria may determine the same level of preference for multiple hypotheses.

Thus, each of the predicates BState and BStateSet has its use case. In highly demanding application domains where a robust symbol anchoring system is considered to be mission-critical and where the planning system expects a single belief state, the strict requirement of a unique belief state appears appropriate but necessitates well-defined preference criteria. Otherwise, designating a small number of hypotheses that equally represent preferable belief states yields a more flexible 


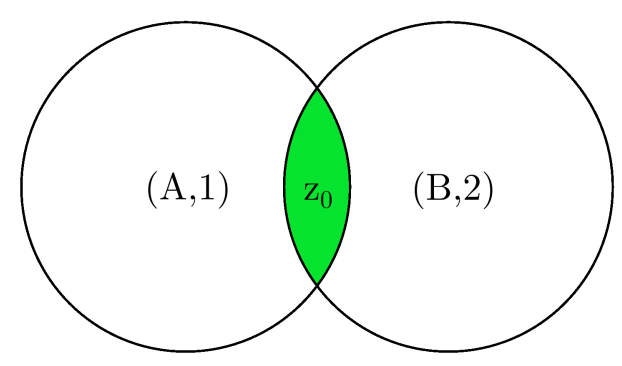

Figure 4.1: Diagram depicting the hypothesis $z_{0}$ of correspondences for objects $A$ and $B$.

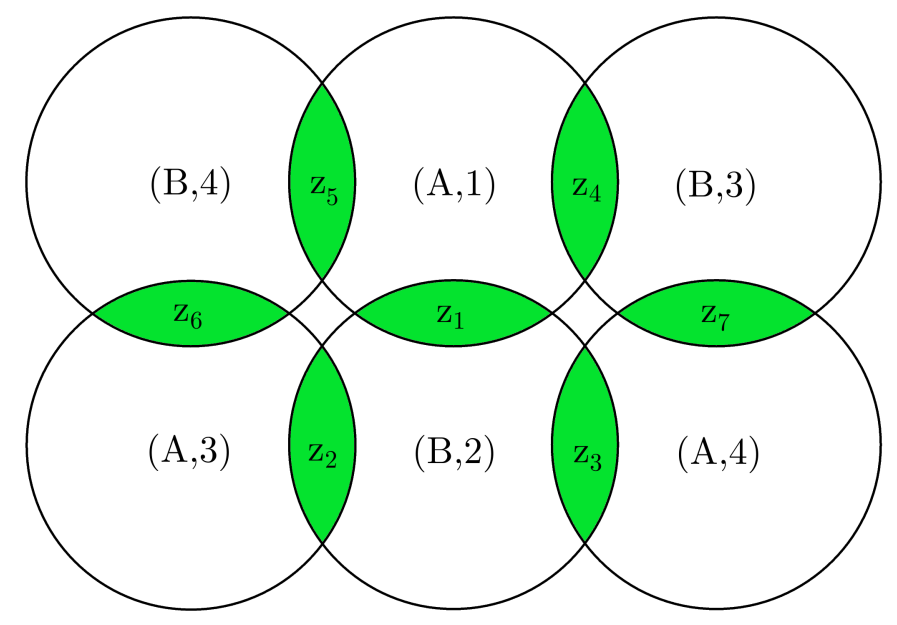

Figure 4.2: Diagram depicting the symbol anchoring hypotheses after reacquiring correspondences.

solution. For example, while a unique, deterministic correspondence of those object symbols of immediate interest is crucial for executing the robot's next actions, the correspondences of the remaining objects do not necessarily have to be uniquely anchored.

No such general, formal characteristics of preference relations among symbol anchoring hypotheses are described in the literature to our knowledge, including those approaches reviewed in Section 2.3.5.

\subsubsection{Heuristics for Preferences}

Next we are going to discuss which rules may constitute appropriate preference criteria for ordering the available hypotheses of correspondences of a given situation. Finding appropriate preference criteria that perform perfectly in any situation and application domain seems to be unfeasible. Instead one ought to consider them as a heuristic guide that tends to perform well in many cases while it may fail in other cases.

Example 4.2 For the purpose of illustration we introduce an example. Let's consider the situation $S$ where the cognitive robot is aware of the two objects $A$ and $B$ that are of the same kind, and where both of them are being tracked such that the percept denoted by identifier 1 is associated with object $A$ and percept 2 is associated with object $B$. These correspondences are represented as the hypothesis $z_{0}$ for situation $S$ in the form of:

$$
(\exists)\left(\operatorname{KState}\left(S, z_{0}\right) \wedge z_{0}=A\left(A, O, \Gamma_{1}, 1\right) \circ A\left(B, O, \Gamma_{2}, 2\right) \circ z \wedge \operatorname{Consistent}\left(z_{0}\right)\right)
$$

The Figure 4.1 illustrates the hypothesis space of the example of Formula (4.41), using one circle for each correspondence, abbreviated by $(A, 1)$ and $(B, 2)$ in the diagram. Both correspondences 
together constitute the consistent and complete hypothesis $z_{0}$ illustrated in Figure 4.1 by the intersection of both circles in green colour. Let's assume that the tracking of both objects failed later due to a temporary obstruction for instance, such that both correspondences were no longer grounded.

Afterwards in situation $S^{\prime}$ suppose that two objects were perceived during object recognition which yielded appropriately matching percepts for both objects $A$ and $B$, such that the new percept bearing identifier 3 may be associated with both object symbols $A$ and $B$, and the new percept 4 likewise represents a potential correspondence for both object symbols. Accordingly, the following holds in this situation:

$$
(\exists)\left(N o P\left(S^{\prime}\right)=2 \wedge P\left(3, O, \Gamma_{3}, S^{\prime}\right) \wedge P\left(4, O, \Gamma_{4}, S^{\prime}\right)\right)
$$

The application of our symbol anchoring axiom, Formula (4.15), in this situation determines several possible correspondences for each percept and for each object symbol utilizing the functionalities Find and New. In particular Find establishes correspondences for percept 3 with objects $A$ and $B$ and similarly for percept 4 with both $A$ and $B$. Additionally, functionality New generates alternative hypotheses of correspondences for each available percept with fresh object symbols 3 and 4. The situation $\operatorname{Do}\left(A n c h o r U\left(O, S^{\prime}\right)=S^{\prime \prime}\right.$ after symbol anchoring is depicted in Figure 4.2 omitting the alternative correspondences with fresh object symbols 3 and 4 for the purpose of readability. Note that the anchors representing the correspondences $(A, 1)$ and $(B, 2)$ are not grounded in this situation $S^{\prime \prime}$ depicted in Figure 4.2.

Similar to the Diagram 4.1, each circle of Diagram 4.2 depicts individual correspondences, while each green intersecting region represents a hypothesis of correspondences. The following Formula (4.42) specifies the complete hypothesis space of the situation $S^{\prime \prime}$ including those correspondences with the fresh object symbols 3 and 4 .

$$
\begin{aligned}
(\exists) & \left(\text { KState }\left(S^{\prime \prime}, z\right) \wedge \text { Consistent }(z) \wedge\right. \\
(z & =A\left(A, O, \Gamma_{1}, 1\right) \circ A\left(B, O, \Gamma_{2}, 2\right) \circ A\left(3, O, \Gamma_{3}, 3\right) \circ A\left(4, O, \Gamma_{4}, 4\right) \circ z_{1 r} \\
\vee & =A\left(A, O, \Gamma_{1,3}, 3\right) \circ A\left(B, O, \Gamma_{2}, 2\right) \circ A\left(4, O, \Gamma_{4}, 4\right) \circ z_{2 r} \\
\vee & =A\left(A, O, \Gamma_{1,4}, 4\right) \circ A\left(B, O, \Gamma_{2}, 2\right) \circ A\left(3, O, \Gamma_{3}, 3\right) \circ z_{3 r} \\
\vee & =A\left(A, O, \Gamma_{1}, 1\right) \circ A\left(B, O, \Gamma_{2,3}, 3\right) \circ A\left(4, O, \Gamma_{4}, 4\right) \circ z_{4 r} \\
\vee & =A\left(A, O, \Gamma_{1}, 1\right) \circ A\left(B, O, \Gamma_{2,4}, 4\right) \circ A\left(3, O, \Gamma_{3}, 3\right) \circ z_{5 r} \\
\vee & =A\left(A, O, \Gamma_{1,3}, 3\right) \circ A\left(B, O, \Gamma_{2,4}, 4\right) \circ z_{6 r} \\
\vee & \left.\left.=A\left(A, O, \Gamma_{1,4}, 4\right) \circ A\left(B, O, \Gamma_{2,3}, 3\right) \circ z_{7 r}\right)\right)
\end{aligned}
$$

The Formula 4.42 enumerates the symbol anchoring hypotheses of situation $S^{\prime \prime}$ in the same order as the subscripts of hypothesis variables $z_{i}$ have been chosen in the Figure 4.2, i. e., Formula 4.42 has the following structure:

$$
(\exists)\left(\operatorname{KState}\left(S^{\prime \prime}, z\right) \wedge \operatorname{Consistent}(z) \wedge\left(z=z_{1} \circ z_{1 r} \vee \cdots \vee z=z_{7} \circ z_{7 r}\right)\right)
$$

The subscripts of the perceptual estimates $\Gamma_{i}$ inside the anchor fluents of the hypothesis space of our example, Formula (4.42), indicate which perceptual information of which percepts have been fused into the perceptual estimate, e.g., the perceptual information of percepts 1 and 4 have been combined to form the perceptual estimate $\Gamma_{1,4}$. Any other intersection that could be obtained from rearranging the circles of Figure 4.2 would form an inconsistent hypothesis with respect to our example.

Based on this example we will propose and discuss heuristics of preferences of hypotheses next.

\subsubsection{Preferring Known Object Symbols}

Let's devise a particular, appropriate preference criterion for ordering all hypotheses of a hypothesis space. For example, a naive heuristic criterion could prefer a correspondence of a certain percept 
with a previously known object symbol to a correspondence with a newly introduced object symbol. This preference criterion may perform well in application domains where most object symbols are known initially, or where rather few new objects are recognized over time in comparison with the number of objects already known to the conservative, cognitive robot. Let KS denote this preference criterion. ${ }^{12}$

Example 4.2 (continued) The result of applying this preference criterion to the hypothesis space of Example 4.2 depicted in Figure 4.2 and specified by Formula (4.42) yields the following hypothesis hierarchy:

$$
\operatorname{PrefSets}\left(S^{\prime \prime},\left\{z_{6}, z_{7}\right\},\left\{z_{2}, z_{3}, z_{4}, z_{5}\right\}\right) \wedge \operatorname{PrefSets}\left(S^{\prime \prime},\left\{z_{2}, z_{3}, z_{4}, z_{5}\right\},\left\{z_{1}\right\}\right)
$$

In other words, Formula (4.43) represents that the hypotheses $z_{6}$ and $z_{7}$ are likewise considered the most preferred hypotheses according to the preference criterion KS, while $z_{1}$ constitutes the least preferred one. Unfortunately, this criterion is unable to strictly order all available hypotheses of the example situation $S^{\prime \prime}$, which is why we used the set-based preference predicate PrefSets instead of the more strict predicate Pref. Hence, both the hypothesis $z_{6}$ and $z_{7}$ are regarded as the belief states of this situation with respect to criterion KS, i. e.

$$
\operatorname{BStateSet}\left(S^{\prime \prime},\left\{z_{6}, z_{7}\right\}\right)
$$

For the purpose of defining the preference criterion KS we introduce the predicate IsNewSymbol. The predicate IsNewSymbol $\left(x, s, z^{\prime}\right)$ holds true iff $x$ is a fresh object symbol in the successor hypothesis $z^{\prime}$ after applying the symbol anchoring axiom in situation $s$.

$$
\text { IsNewSymbol }: \text { PERCID } \times \text { SIT } \times \text { STATE }
$$

$$
\begin{aligned}
& \text { IsNewSymbol }\left(x, s, z^{\prime}\right) \equiv \\
& (\exists)\left(\operatorname{KState}\left(\operatorname{Do}(\operatorname{Anchor} U(o), s), z^{\prime}\right) \wedge \operatorname{Knows}(\operatorname{Offset}(t), s) \wedge\right. \\
& \left.\quad \operatorname{Holds}\left(A(x, o, \Gamma, \pi), z^{\prime}\right) \wedge x>t\right)
\end{aligned}
$$

Based on the predicate IsNewSymbol we introduce the auxiliary preference predicate PrefPercKS that determines the preference with respect to a certain percept.

$$
\text { PrefPercKS }: \text { SIT } \times \text { STATE } \times \text { STATE } \times \text { PERCID }
$$

$$
\begin{aligned}
& \operatorname{PrefPercKS}\left(s, z_{1}, z_{2}, \pi\right) \equiv \\
& \quad(\exists)\left(\operatorname{Holds}\left(A\left(x, o, \Gamma_{x}, \pi\right), z_{1}\right) \wedge \neg \operatorname{IsNewSymbol}\left(x, s, z_{1}\right) \wedge\right. \\
& \left.\operatorname{Holds}\left(A\left(y, o, \Gamma_{y}, \pi\right), z_{2}\right) \wedge \operatorname{IsNewSymbol}\left(y, s, z_{2}\right) \wedge P(\pi, o, \Gamma, s)\right)
\end{aligned}
$$

A suitable implementation of the criterion KS could be based on the predicates IsNewSymbol and PrefPercKS providing a starting point for the application programmer. However, two disadvantages of the preference criterion KS should be discussed, which the criterion MRM presented below is intended to overcome. First, preferring a correspondence of a percept with a known object symbol lacks causal plausibility, because there may be good reasons for preferring a different hypothesis, which the criterion KS neglects. Second, the predicate PrefPercKS orders hypotheses of correspondences with respect to a single percept only, thus constitutes a local preference criterion. It appears rather difficult to extend the scope of this preference predicate towards all correspondences of a certain situation. To our knowledge no approach of the literature employs a similar preference criterion.

\footnotetext{
${ }^{12} \mathrm{KS}$ stands for preferring known object symbols.
} 


\subsubsection{Preferring the Most Restricted Matching Correspondence}

Due to different kinds of objects bearing individual characteristics and due to the robot's sensors yielding various degrees of precision depending on individual perceptual attributes and sensor devices, the precision and coverage of information which each percept provides about a physical object differs significantly. These and other factors directly influence the perceptual estimates inside anchor fluents as a result of integrating percepts. Moreover, the specificity of symbolic descriptions of objects may differ considerably. Hence, when our symbol anchoring approach determines all matching correspondences of percepts and symbolic descriptions of objects, certain correspondences may match rather precisely, while others may match only vaguely.

Such conditions appeal to the following preference criterion among alternative correspondences, which is independent of the application domain. Generally speaking, we propose a criterion which favours the more restricted matching correspondences over those that match only vaguely. This way the criterion minimizes the risk that a correspondence was established due to the lack of precise information, which would have to be invalidated upon additional, inconsistent information later. This heuristics is based on the following observation: The more closely a percept matches the anchor, the smaller is the risk of a correspondence to become extinct due to inconsistent information. Let MRM denote this preference criterion. ${ }^{13}$

Example 4.3 Consider for example a cognitive robot which knows about two different objects of the same kind, say hats. Let $H_{r}$ be the object symbol denoting the first hat of red colour, and let $H_{2}$ denote the second one of unknown colour. Now, assume the robot perceived a hat of red colour. Our symbol anchoring approach determines three possible hypotheses of correspondences of the given percept with object symbols in this situation. First, functionality New introduces a new correspondence of the percept with a fresh object symbol, because the perceived hat does not necessarily correspond with one of the two known hats. Furthermore, functionality Find establishes one correspondence of the given percept with each of the two known hats, because both the red hat $H_{r}$ and the other hat $H_{2}$ match the percept. According to the concept of preferring the most restricted matching correspondence, the preference criterion MRM favours the correspondence with the hat $H_{r}$ over the correspondence of with the hat $H_{2}$. Apparently, the correspondence of hat $\mathrm{H}_{2}$ with the perceived red hat bears the risk of becoming inconsistent in case the robot would learn later that the colour of hat $H_{2}$ is actually not red. Conversely, the correspondence of hat $H_{r}$ with the present percept does not bear this risk.

Recall from the definition of predicate Match, Formula (4.20), which sources of information are consulted by the symbol anchoring functionalities in order to decide whether the considered object symbol may be anchored with the considered percept. Looking at the perceptual end of a potential correspondence, the percept's signature provides information about those perceptual attributes measured by the sensing action. On the other side, perceptual information about percepts associated so far has been integrated in the perceptual estimate of the anchor. Furthermore, the symbolic description of an object represents the symbolic knowledge about an object. Both anchor and symbolic description taken together form the counterpart of a correspondence with respect to a percept. Accordingly, the following aspects serve to particularize the preference criterion MRM.

- The more detailed and precise the symbolic description of an object is, the fewer percepts match this description, and hence the fewer correspondences may turn out to be actually mistaken later. A detailed symbolic description is one where only few perceptual attributes are not specified vaguely or not at all.

- Similarly for the percepts: The smaller each uncertainty interval of the perceptual signature of a perceived object is, the fewer perceptual predicates of a symbolic description may match the perceptual signature. Additionally, the more perceptual attributes a percept provides, the more distinctive the perceived object becomes and the fewer symbolic descriptions and perceptual estimates match this percept.

\footnotetext{
${ }^{13} \mathrm{MRM}$ abbreviates the preference for the most restricted matching correspondence.
} 
Note that the preference criterion MRM might be mistaken anyway, and shall be considered as a heuristic guide only, just like any other heuristic preference criterion. The scope of the preference criterion MRM is rather limited. While it could possibly be generalized to analysing multiple perceptual attributes, it seems difficult to evaluate all correspondences of a whole hypothesis. To our knowledge this preference criterion has not been employed in other approaches in the literature.

\subsubsection{Preferring Correspondences with Least Commitment}

Still, the above-mentioned criterion MRM bears some drawbacks. It seems not advantageous in all application domains to prefer correspondences with an extremely restricted specification on the one side of a correspondence, if only a vague description is available on the other side. Alternatively, we propose a kind of least commitment strategy for a preference criterion, which tends to balance the precision of information available at both sides of a correspondence. The implementation of this concept requires that the different sources of information are transformed into the same coordinate space in order to become comparable. For this purpose, the symbolic description is mapped onto perceptual ranges using the grounding relation as explained in Section 3.6. The perceptual description gained this way and the perceptual estimate of the anchor fluent of the same object together form the sole model of the object to be considered. We propose a leastcommitment criterion - LC for short - that favours those correspondences over others where the difference between the perceptual uncertainty intervals of the symbolic model and of the perceptual signature for each perceptual attribute is small. In other words, the boundaries of the perceptual representation of the symbolic description on the one side, and the boundaries of the perceptual intervals measured of the physical object on the other side should preferably bear similar values such that the uncertainty represented tends to be similar.

For example, if the symbolic model poses no restriction on the perceptual values of a certain attribute, then this agrees well with a percept which bears a very high uncertainty for the perceptual values of the same attribute or which has not yet been measured at all. This concept improves on the criterion MRM described in Section 4.3.3.2 above. Conversely, say the symbolic model of an object specifies a certain attribute of an object precisely. Then, this does not constitute a good match with a percept that bears rather vague information about the same perceptual attribute. The latter example shows the case of unnecessary commitment to certain characteristics that is not shared by both sides of the correspondence. The early, superfluous commitment poses the potential risk of considering an actually mistaken correspondence.

Similar to the criterion MRM, our criterion LC regards only individual perceptual attributes. Its scope should be extended to all attributes of a correspondence in order to evaluate a complete hypothesis, which seems to be rather difficult. To our knowledge this preference criterion has not been proposed or employed yet in the literature on symbol anchoring.

\subsubsection{Global Heuristic Preference Criteria}

The respective scopes of the local, heuristic preference criteria KS, MRM and LC described above are too limited in order to evaluate all correspondences of a certain hypothesis as a whole. Due to our fundamental concept to consider all objects of the same category of interest (cf. Section 3.3), a preference criterion may restrict its attention to those objects of the considered category, because only percepts of this category were requested from the object recognition system. Hence, other objects belonging to any other category than the one of current interest cannot be anchored in the current situation anyway.

Restricting the focus of the preference criterion to a single object only would contradict the objectives of our approach, which is intended to minimize the potential ambiguity of symbol anchoring by taking all currently perceivable objects of the same kind into account (cf. Section 3.3). Apparently, comparing a certain correspondence candidate with all the other potential correspondence candidates yields a much better, global indicator for the most suitable hypothesis. Moreover, the robot's actions may actually require to anchor multiple objects at once, e.g., if the robot's task is to stack one block on top of another block, which in turn is situated on top of yet another 
one. Such tasks typically require all involved objects of immediate interest to be grounded appropriately in order to ensure the robustness of the robot's plan and the precision of subsequent actions.

Example 4.3 (continued) Let's resume our above-mentioned example on anchoring multiple hats in the world with the objective of comparing our preference criteria. Say, while the robot knows about the hats $H_{r}$ and $H_{2}$, whereof the hat $H_{r}$ is known to bear the colour red, the robot's object recognition yields two percepts of hats in the world. The percept 1 stands for a hat of red colour, and percept 2 signifies a big hat of unknown colour. Given this information, the symbol anchoring functionality Find determines two hypotheses of correspondences of the two percepts with the known object symbols $H_{r}$ and $H_{2}$, and functionality New adds several alternative hypotheses assigning fresh object symbols to the percepts. Regarding the former two, the functionality Find determines the hypothesis comprising the correspondences $\left(H_{r}, 1\right)$ and $\left(H_{2}, 2\right)$, and the second hypothesis comprising the correspondences $\left(H_{r}, 2\right)$ and $\left(H_{2}, 1\right)$.

The application of the least-commitment criterion (LC) of Section 4.3.3.3 turns out to prefer the first hypothesis with $\left(H_{r}, 1\right)$ and $\left(H_{2}, 2\right)$, where the red hat's object symbol corresponds to the percept of a red hat, and where the other hat's symbol corresponds to the percept of a big hat. Probably any rational cognitive robot would agree with this preference. Using the preference criterion MRM instead yields the same result as criterion LC in this case. Conversely, the preference criterion KS preferring correspondences with known object symbols favours the two hypotheses generated by functionality Find likewise over those generated by functionality New.

Example 4.4 The following example ought to sustain our argument for a global preference criterion regarding all current percepts and objects of the category of interest. Imagine a situation wherein a cognitive robot knows of five objects of the same kind denoted by the symbols $O_{1}, \ldots, O_{5}$, and wherein the robot's recognition system is tracking the objects $O_{1}, \ldots, O_{4}$. Only object $O_{5}$ has not been perceived yet, such that its perceptual signature is rather vague in contrast to those of objects $O_{1}, \ldots, O_{4}$. Suddenly the tracking of objects $O_{1}, \ldots, O_{4}$ failed. Shortly afterwards four objects were recognized again yielding the percepts $\pi_{1}, \ldots, \pi_{4}$ each of which matching each of the known objects $O_{1}, \ldots, O_{5}$. But among the possible hypotheses, the one representing the correspondences $\left(O_{i}, \pi_{i}\right)$ for $i=1 \ldots 4$ provided the best matching correspondences and thus should be considered the current belief state, i. e. the most preferred hypothesis.

Now consider that the cognitive robot intended to anchor object $O_{5}$ originally and therefore requested percepts for objects of its kind. We argue that it is plausible to prefer the hypothesis of correspondences $\left(O_{i}, \pi_{i}\right),\left(O_{5}, \perp\right)$ for $\pi_{i} \neq \perp$ and $i=1 \ldots 4$ in this situation due to well matching object-percept pairs, although alternative hypotheses existed with $\left(O_{5}, \pi_{i}\right)$ and $i=1 \ldots 4$. In other words, it seems not rational to simply prefer the hypothesis where especially the object of interest is grounded, just because is not anchored in other hypotheses. If there are good reasons for the preference criterion to associate a certain hypothesis with the belief state of the current situation, then this hypothesis seems to be the most potential candidate.

Recall that our symbol anchoring functionalities Find and Track verify whether all available symbolic and perceptual information about an object and its corresponding percept match consistently in order to ensure the validity of this correspondence (cf. Sections 4.2.2 and 4.2.3). Only static perceptual attributes are consulted by Find and Track, because only those attributes serve to strictly constrain a match of a correspondence - in contrast to dynamic perceptual attributes. However, dynamic attributes of objects could guide the heuristic preference criterion to some extent-at least temporarily. Such a preference criterion could help to overcome the short outage of tracking in the above Example 4.4 about anchoring the objects $O_{1}, \ldots, O_{5}$, for instance.

An interesting related work is the way how Shanahan [2005] suggested to compute the explanatory value of individual hypotheses in order to select the one which explains the given sensor data best. After determining the expectations of an hypothesis, the perceptual data are reconsulted and the explanatory value of the hypothesis is refined. The result is an ordering among the available hypotheses. It seems to be beneficial for the planning and symbol anchoring systems of a cognitive 
robot to incorporate such abductive methods of reasoning, which are-however-out of the scope of our work at hand.

In summary, we have introduced general properties of preferences of hypotheses as well as three particular preference criteria for evaluating the hypothesis space as a starting point. The purpose of the preference criteria is to sort all hypotheses of a certain situation automatically in order to determine the belief state. The proposed criteria do not depend on specific application domain features.

\subsection{Summary and Conclusion}

We have presented our uni-modal approach to symbol anchoring in this chapter. The restriction of this approach to a single sensor device (or alternatively assuming that the object recognition system performs sensor fusion for all sensors) allowed us to develop a less complex solution, which is easier to explain and to understand, and yet provides the full range of functionalities required. Coradeschi and Saffiotti [2002] named a number of functional requirements of any approach to the symbol anchoring problem, which we included in Section 2.3.4. We have pointed out in this chapter which particular part of our approach satisfies which functional requirement. Altogether, our approach fulfils all the requirements specified by Coradeschi and Saffiotti [2002]. Our approach is based on first-order logic and the Fluent Calculus in particular, which facilitate an unmatched expressiveness with respect to reasoning about knowledge and actions of a cognitive robot.

In contrast to other approaches to the symbol anchoring problem, all potential hypotheses of correspondences between all objects and all percepts of the category of interest are determined and maintained automatically in our approach. A prime example sustaining this concept is how humans achieve disambiguation in natural language as one step of everyday communication [Russell and Norvig, 2003]. Here, all possible interpretations of a given sentence within a given context are generated for the purpose of analysis. If more than one interpretation applies, then disambiguation chooses the one that fits the situation and context best. Symbol anchoring in cognitive robotics is related to resolving references in the field of natural language processing. In this field, a pronoun or a definite noun phrase referring to an object of the world is resolved based on the knowledge about the world and on the given situation.

Our uni-modal symbol anchoring approach incorporates a number of fundamental concepts and assumptions explained in Chapter 3. In summary, we presented how our approach handles incomplete information about objects on both the symbolic level and the perceptual level. Our main objectives included to develop a general, domain-independent approach. Therefore, no heuristics or planning of sensing actions are involved. An anchor represents the correspondence between an object identifier denoting an individual object and the associated percept of a physical object. The separation of the symbolic and perceptual levels and the fact that the correspondences are not given nor guaranteed to be correct, demand for a clear distinction between the symbolic and the perceptual information. Our approach takes the independence of both levels and of the sources of information into account explicitly. This demonstrates exemplarily that our approach complies with the sense-data theory of philosophy of cognition.

Categories of objects serve the purpose of focusing the object recognition and symbol anchoring on the kind of objects of current interest. Specific characteristics of categories of objects serve as valuable information and constraints, too. An important concept underlying our approach is to maintain all correspondences of objects of the category of interest simultaneously, this way providing a much more complete model of the world than just focusing on a single object. Also contributing to resolving ambiguity, our approach guarantees to assign each percept an individual object symbol. ${ }^{14}$ This concept serves to take each potential correspondence into account.

Special attention was devoted to maintaining perceptual uncertainty of measurements and of knowledge explicitly and to utilizing all available perceptual information to the greatest extent. The objective hereof is to minimize the number of potential candidates of correspondences between object identifiers and percepts as much as possible. For this purpose individual pieces of sensory

\footnotetext{
${ }^{14}$ The proof of our approach complying with this concept will follow in the next chapter (cf. Section 5.6.3).
} 
information on individual objects are fused in the anchor over time and serve to determine whether or not the correspondences remain consistent.

We have implemented a basic and static approach to the symbol grounding problem with regard to perceptual attributes. A similar solution was typically chosen by other approaches to symbol anchoring in the literature, too. However, the rich expressiveness of the first-order logic language underlying our approach facilitates various kinds of references to objects, in particular symbolic descriptions, definite and indefinite references. Our approach employs the general concept of a knowledge state of the Fluent Calculus in order to represent and to maintain multiple hypotheses of correspondences between object identifiers and percepts simultaneously. All perceptual and symbolic information is employed for sustaining consistent hypotheses and for identifying inconsistent hypotheses automatically. Upon eliminating an inconsistent hypothesis, our approach requires no backtracking and no maintenance in order to update the remaining world model, since all other hypotheses persist independently. In this way, new pieces of symbolic and perceptual information function as epistemic constraints on the possible hypotheses in our approach, similar to the system "DyKnow" of Heintz and Doherty [2004b].

The computational core of our approach consists of three symbol anchoring functionalities, each of which is applicable in a certain state of the considered correspondence. First, a yet unknown object is recorded and a new object identifier is assigned to the percept. Second, percepts of objects being tracked by the recognition system provide new perceptual information for updating associated anchors. Third, after tracking of an object failed, the respective correspondence has to be reacquired given a new percept of the same object of the environment. Basically the same tasks have been addressed in other approaches to the symbol anchoring problem, but each one in a very different way. Note that our declarative, formal approach applies the appropriate functionality automatically in any situation in contrast to other approaches, the majority of which are procedural implementations. As a result we have developed a domain-independent approach to the symbol anchoring problem for cognitive robots, which interfaces with the object recognition system and solves all fundamental tasks of symbol anchoring automatically. 


\section{Chapter 5}

\section{Multi-Modal Symbol Anchoring}

In this chapter we are going to present our multi-modal symbol anchoring approach-the generalization of the uni-modal version described in Chapter 4-aiming at a wider flexibility and applicability in typical cognitive robotics systems. This version takes multiple different sensor devices into account in particular. Both our uni-modal and multi-modal approaches share the same fundamental concepts and assumptions described in Chapter 3 as well as the same basic objectives. However, the extension to multiple sensor devices complicates the approach. On a side note, developing two different approaches allowed us to verify and to compare them against each other. We will prove that the uni-modal approach implies the respective multi-modal axioms. Hence, both approaches share a common set of crucial characteristics.

\subsection{Motivation}

Our uni-modal symbol anchoring approach presented in Chapter 4 implements symbol anchoring for cognitive robots maintaining multiple, alternative hypotheses in parallel, each representing correspondences of multiple objects. However, the uni-modal approach bears one crucial limitation with respect to its applicability in practical, modern cognitive robotics systems: The uni-modal approach is designed for perception by means of a single sensor device only. Conforming to the understanding of the symbol anchoring community, we define a percept as a structured collection of sensory data originating from the same physical object (cf. Section 2.3.2). Modern cognitive robots are typically equipped with multiple, different sensor devices that complement each other in terms of modality, precision and range of perception, in order to be able to maintain a high-fidelity world model (cf. e.g. [Rasmussen, 2002, Fichtner et al., 2003, Broxvall et al., 2005]). Sensing actions typically only request new perceptual information about the environment from an individual sensor at once for the purpose of efficient and effective object recognition. Significantly varying costs of perception of different sensing devices appeal to individual control of each sensor especially. For instance, sensing the odour of a substance by means of an electronic nose and recovering from the measurement until the olfactory device is ready for the next measurement may take up to several minutes [Loutfi, 2003]. Also, each measurement consumes part of the life-time of such a device due to its sensitivity. In contrast, a two-dimensional laser range finder is able to yield a whole measurement within negligible time. This demonstrates the need for a symbol anchoring approach that takes individual, asynchronous control of multiple, different sensing devices into account. As multiple percepts originating from the same physical object may be measured by multiple sensors in the same situation - called multi-modal perception - in such a setting in general, the approach presented in this chapter combines multi-modal perception and symbol anchoring.

More precisely, the uni-modal symbol anchoring approach of Chapter 4 is based on the fundamental concept that a percept corresponds uniquely with a certain physical object, which the percept originates from, and thus also corresponds uniquely with a certain object identifier in any situation. The Figure 5.1 illustrates the unique role of a percept in the case of uni-modal percep- 


$$
\# O_{1} \# \pi_{1} \longrightarrow O_{1}
$$

Figure 5.1: Uni-modal symbol anchoring: Solely the percept $\pi_{1}$ mediates in the correspondence between the physical object $O_{1}$ (right hand side) and its symbolic representation (left hand side) denoted by $\# O_{1} \#$.

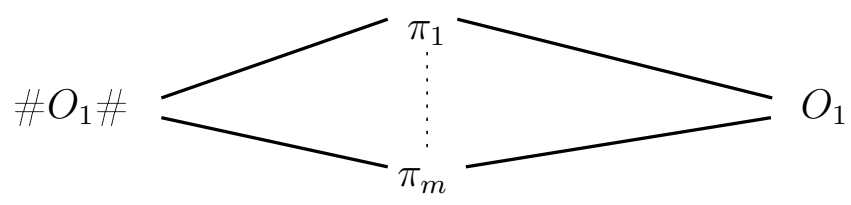

Figure 5.2: Multi-modal symbol anchoring: A number of percepts, $\pi_{1}, \ldots, \pi_{m}$, mediate in the correspondence between the representation $\# O_{1} \#$ of the object $O_{1}$ and the physical object itself.

tion. We adopted the notation of Jackson and Sharkey [1996] signifying that \#thing\# means the representation of "thing", but not the physical "thing" itself. In particular, \# $O_{1} \#$ stands for the object identifier used to represent the object $O_{1}$ of the world on the deliberative level.

In contrast to the uni-modal approach, the multi-modal symbol anchoring approach of Chapter 5 allows to establish a unique correspondence between a certain physical object and a certain object identifier by means of multiple percepts at the same time. While each percept of a certain \#object\#-to-object correspondence was measured by a different sensor, all of these percepts originate from the same physical object, which in turn is represented by a single object identifier. The Figure 5.2 illustrates that the uniqueness of a correspondence is retained in the multi-modal case. If no percept mediates in the correspondence then the object identifier is simply not grounded, just as in the uni-modal case before. The multi-modal symbol anchoring approach gains additional flexibility in comparison with the uni-modal approach: It is sufficient for continuous tracking of an object that some sensor perceives the object within its sensing range, distributing the responsibility of successfully tracking an object.

Given several sources of sensory information about the same object, our multi-modal symbol anchoring approach performs sensor fusion on the symbolic level. In general, sensor fusion denotes "the problem of combining potentially conflicting data from multiple sources into a single model of some aspect of the world" [Clark and Yuille, 1990]. In our approach in particular, the use of symbolic models for objects of the world facilitates the fusion of sensor data originating from various kinds of sensors, as well as the fusion among different cognitive agents situated in the real world (cf. [Bonarini et al., 2001b]). On the one hand sensor fusion combines measurements of different perceptual attributes from different sensors (or even from different agents) into a single object model. On the other hand common subsets of perceptual attributes measured by different sensors can be fused effectively into a single object model such that individual sensor types may complement each other in terms of precision and range. For instance, while the distance of the robot to a certain obstacle can only be measured roughly by means of a standard camera sensor, the combination with the percept of a laser range finder typically yields much more precise distance information. In summary, our multi-modal symbol anchoring approach performs the following tasks subsequently:

1. All percepts that originate from the same physical object have to be determined as being related to each other. If the object recognition system does not indicate an association with some previous percept, it's up to the symbol anchoring system to handle the resulting ambiguity correctly.

2. Given the set of associated percepts originating from the same physical object, the various pieces of perceptual information are to be fused into a single object model.

3. Next, given the model of each perceived physical object, our symbol anchoring approach 
determines all possible hypotheses of potential correspondences between object symbols and perceptual models in accordance with the robot's current knowledge. This task verifies implicitly whether the symbolic information, which may have been augmented since the latest symbol anchoring update, still matches the new perceptual object model consistently.

4. For each new hypothesis of correspondences and for each correspondence in turn, the symbol anchoring axioms update the respective anchor fluents incorporating the new perceptual information.

The third and the fourth step constitute the major tasks of symbol anchoring, which our uni-modal approach accomplishes, too. But only multi-modal perception necessitates the first two steps of the above distinction, the solution of which we will present below.

The uni-modal version of our symbol anchoring approach serves the purpose of explaining the fundamental concepts in Chapter 4. The multi-modal symbol anchoring approach is based on the same prerequisites described in Chapter 3 as the uni-modal version. While the formulas are different and more complex, we will prove below that the uni-modal version is a special case of our multi-modal symbol anchoring approach. Let's start with the differences in the interface with the object recognition system.

\subsection{Object Recognition and Tracking}

Being sensed and reported individually, the multi-modal percepts bring about an additional combinatorial association problem due to the many-to-one relation between percepts and physical objects in contrast to the one-to-one relation in the uni-modal case (cf. Figures 5.1 and 5.2). Fortunately, typical object tracking systems maintain spatial representations internally for measuring and predicting the positions and further phenomenal characteristics of perceived objects over time (cf. e.g. [Schulz et al., 2001]). This should enable the object recognition and tracking system to determine easily which percepts among those currently perceived refer to the same physical object like other current or previous percepts. Thus, typical tracking systems seem to be capable of solving the data association problem among separate percepts to some extent. While this functionality is desirable, it is not strictly required by our approach. As you will see below, the functionality Find is designed to determine all corresponding percepts which the recognition system failed to associate. In comparison, other symbol anchoring approaches assume the data association problem of multi-modal object recognition to be solved completely, e. g. [Bonarini et al., 2001a].

Of course, all sensors involved in low-level data association need to share a common spatial and temporal range of perception at least partially. A typical cognitive robot being equipped with a number of sensor devices on the same mobile platform usually fulfils this requirement for subsets of its sensors (cf. e.g. [Fichtner et al., 2003]). Only a few kinds of sensor devices do not employ an internal spatial representation and do not allow to locate or distinguish several objects within the sensor's range spatially, e. g. an olfactory sensor. Imagine a cognitive robot, being equipped with a laser range finder and a camera, perceives a certain object in its environment, for instance. After sensing and tracking the object by means of the robot's camera, a laser measurement is requested. Given that the internal positional estimates of the object by both the recent camera percept and the new laser percept coincide, the new laser percept affords to be associated with the previous camera percept by the recognition system.

Our approach is designed to make use of all the associative information reported by the recognition system accordingly. The object recognition system reports the associative information in form of a reference percept identifier. We assume this associative information to be correct, as our approach does neither maintain probabilities nor revises beliefs (cf. Chapter 3). Thus, the recognition system is required to report associative information only if the validity is ensured in accordance with our fundamental concepts described in Section 3.11. Conversely, the absence of an associated reference percept does not definitely mean that the reported percept stands for a dissociated, yet unseen object of the world. In this case the recognition system simply failed to 
determine the relation between all involved percepts reliably. Although this case poses an additional, combinatorial association problem, a symbol anchoring system shall be able to handle it in general. Below we will present our solution to this task, which also handles this particular case. Basically, each additional, possible correspondence between an object symbol and an appropriate percept introduces a new hypothesis in our approach.

Reference percepts may have been measured in the current or in a previous situation. Ideally, the reference percept reported for a certain physical object is the one that the recognition system was tracking for the longest period of time. In order to enable the symbol anchoring functionality of tracking an object, the reference percept has to originate from a situation before the most recent symbol anchoring transaction or from the situation of the most recent anchoring transaction itself. Since our multi-modal symbol anchoring approach stores all percept identifiers that correspond to the same object including those of reference percepts, anchors facilitate to determine which object symbol a new percept corresponds to.

These multi-modal concepts of symbol anchoring will become clearer in the subsequent sections. In contrast to the object recognition system, the symbol anchoring system allows to track and associate various percepts from different sensors over extended periods of time even if substantial outages of tracking occur. Moreover, the recognition system cannot perform symbol anchoring and object identification per se, of course.

\subsection{Representation and Meaning}

This section introduces the representation and its meaning of our multi-modal symbol anchoring approach. The definitions and axioms of the uni-modal approach presented in Chapter 4 do not apply in the multi-modal approach below in general, if not otherwise indicated. However, both our uni-modal and the multi-modal approach share the common foundation laid in Chapter 3.

\subsubsection{Percepts}

Recall that a collection of sensory data originating from the same physical object constitutes a percept. In the multi-modal case particularly, a percept denotes the sensory data measured of a certain physical object by a certain sensor device in a certain situation. If several sensors recognized a certain physical object in the same situation, multiple percepts originating from that object are reported accordingly.

Our multi-modal symbol anchoring approach represents a percept provided by the recognition system by means of the predicate $P$ with

$$
P: \text { PERCID } \times \text { OBJCAT } \times \text { PERCSIG } \times \text { PERCID } \cup\{\perp\} \times \text { SIT }
$$

In summary, the percept predicate $P\left(\pi, o, \Gamma, \pi_{R}, s\right)$ represents that an object of category $o$ has been recognized in situation $s$ and named after identifier $\pi$. The perceptual signature $\Gamma$ specifies the perceptual information in terms of the attributes measured of the physical object. In comparison with the uni-modal version, the multi-modal percept predicate $P$ bears the additional, fourth parameter standing for the reference percept (cf. Definition (4.1)). If the recognition system determined that the percept at hand refers to the same physical object like another percept (of the same or, preferably, a previous situation), the parameter of the reference percept holds the identifier of the associated percept; otherwise the reference percept is undefined and holds the constant $\perp$. This way, individual percepts measured of the same physical object by separate sensor devices can be grouped together for joint anchoring, which facilitates to reacquire the associated percepts subsequently.

The extension of the multi-modal percept predicate with respect to the uni-modal version necessitates to adapt all the assumptions and axioms involved. The following axioms resemble their respective uni-modal counterparts (cf. Section 4.1.1).

Percepts bear unique identifiers within the same situation, which are to be reused by the object recognition system during tracking the same object. The following formula implements this 
assumption of uniqueness and extends the corresponding uni-modal Formula (4.6).

$$
P\left(\pi, o_{1}, \Gamma_{1} \pi_{R 1}, s\right) \wedge P\left(\pi, o_{2}, \Gamma_{2}, \pi_{R 2}, s\right) \supset o_{1}=o_{2} \wedge \Gamma_{1}=\Gamma_{2} \wedge \pi_{R 1}=\pi_{R 2}
$$

The percepts reported by the object recognition system convey measurements of perceptual attributes of certain objects of the environment. Thus we may assume a non-empty perceptual signature, similar to the uni-modal Formula (4.3).

$$
P\left(\pi, o, \Gamma, \pi_{R}, s\right) \supset \Gamma \neq \emptyset
$$

The tuples of values within perceptual signatures have to be ordered allowing for easier formulas in the sequel. The same requirement was specified in the uni-modal case in Formula (4.4).

$$
P\left(\pi, o, \Gamma, \pi_{R}, s\right) \wedge(a,(b, t)) \in \Gamma \supset b<t
$$

Our approach assumes uni-modal and one-dimensional distributions of values for perceptual attributes represented inside the percepts, as has been explained in Chapter 3. In particular, our approach requires the perceptual signature to contain at most one range of values for each perceptual attribute measured. This means formally:

$$
P\left(\pi, o, \Gamma, \pi_{R}, s\right) \wedge\left(a,\left(b_{1}, t_{1}\right)\right) \in \Gamma \wedge\left(a,\left(b_{2}, t_{2}\right)\right) \in \Gamma \supset b_{1}=b_{2} \wedge t_{1}=t_{2}
$$

The multi-modal symbol anchoring approach shares the function $N o P$ with the uni-modal version (cf. Section 4.1.1). This function determines the number of percepts that the recognition system reported in the given situation.

$$
\text { NoP : SIT } \mapsto \mathbb{N}
$$

The Function NoP assumes the number of percepts to be finite in any situation, which certainly holds for practical recognition systems. All reported percepts are distinct, bear individual identifiers and are represented as percept predicates of the same object category in the considered situation. Adapting the uni-modal Formula (4.7), this requirement is stated as follows.

$$
\begin{gathered}
(\forall s)(\exists ! k, o)\left(N o P(s)=k \wedge(\exists)\left(P\left(\pi_{1}, o, \Gamma_{1}, \pi_{R 1}, s\right) \wedge \cdots \wedge P\left(\pi_{k}, o, \Gamma_{k}, \pi_{R k}, s\right) \wedge\right.\right. \\
\left.\left.\bigwedge_{i=1}^{k-1} \bigwedge_{j=i+1}^{k} \pi_{i} \neq \pi_{j}\right)\right)
\end{gathered}
$$

Before we continue with describing how percepts are analysed with respect to multi-modal symbol anchoring, the next section introduces the remaining pieces of the representation utilized by our approach.

\subsubsection{Correspondences and Anchors}

Objects of the environment are assigned individual identifiers on the symbolic level. Similar to our uni-modal approach, a priori known objects bear names of the sort $\mathcal{X}$ and other objects experienced by the cognitive robot at runtime are assigned identifiers of the sort PERCID automatically. Both sorts of object identifiers have to be disjoint in order to allow uniqueness of identifiers.

$$
\mathcal{X} \cap \operatorname{PERCID}=\emptyset
$$

The anchor fluent represents correspondences between percepts of a physical object and an object symbol. Instead of just a single percept corresponding with the object symbol at hand like in the uni-modal approach (cf. Definition (4.8)), the multi-modal anchor fluent stores the set of identifiers of all previously corresponding percepts. If the recognition system reports a previous reference percept along with a current percept, the method of storing the set of all percept identifiers previously corresponding with a certain object facilitates ready and definite determination of the object's correspondences, as described in Section 5.2. The signature of multi-modal anchor fluents is defined as:

$$
A: \mathcal{X} \cup \text { PERCID } \times \text { OBJCAT } \times \text { PERCSIG } \times \mathcal{P}(\text { PERCID }) \mapsto \text { FLUENT }
$$


The set of previous reference percepts represented as the fourth parameter of anchor fluents equals the empty set in the initial situation. Like in the uni-model version in Section 4.1.2, the third parameter of fluent $A$ of sort PERCSig denotes the so-called best perceptual estimate of an object and holds an aggregate data structure. This component specifies all perceptual information gathered so far about a certain object fused into a single, perceptual object model. The best perceptual estimate uses the same representation as the perceptual signature of the percept predicate $P$, but applies a different meaning to it. The sort PERCSIG is defined as:

$$
\text { PERCSIG } \stackrel{\text { def }}{=} \mathcal{P}((\text { ATTR } \times(\mathbb{R} \times \mathbb{R}))
$$

Our multi-modal symbol anchoring approach requires that no anchor fluent with object identifiers among the sort PERCID exists initially, because these identifiers are reserved solely for denoting objects which are initially unknown. For this purpose, the following multi-modal condition adapts the uni-modal Formula (4.10).

$$
(\forall z, x: \operatorname{PERCID})\left(\operatorname{KState}\left(S_{0}, z\right) \supset \neg(\exists o, \Gamma, \Pi)(\operatorname{Holds}(A(x, o, \Gamma, \Pi), z))\right)
$$

Apart from the sort PERCID our approach assigns object symbols of sort $\mathcal{X}$. Similar to the uni-modal Formula (4.11) we require the initial existence of anchor fluents for all objects with symbols in $\mathcal{X}$ for the purpose of simplifying the below formulas. These anchor fluents hold empty perceptual estimates and an empty set of previously corresponding percepts.

$$
(\forall z, x: \mathcal{X})\left(\operatorname{KState}\left(S_{0}, z\right) \supset(\exists ! o, \Gamma, \Pi)(\operatorname{Holds}(A(x, o, \Gamma, \Pi), z) \wedge \Gamma=\emptyset \wedge \Pi=\emptyset)\right)
$$

In accordance with the fundamental concepts described in Chapter 3, our multi-modal symbol anchoring approach requires all hypotheses to be consistent. The predicate Consistent defines the consistency criteria for a hypothesis to require first, uniqueness of object symbols, and second, uniqueness of percept identifiers in the anchor representation of the correspondences as follows.

Consistent : STATE

$$
\begin{aligned}
& \text { Consistent }(z) \equiv \\
& \quad\left(\operatorname{Holds}\left(A\left(x, o_{1}, \Gamma_{1}, \Pi_{1}\right), z\right) \wedge \operatorname{Holds}\left(A\left(x, o_{2}, \Gamma_{2}, \Pi_{2}\right), z\right)\right. \\
& \left.\quad \supset o_{1}=o_{2} \wedge \Gamma_{1}=\Gamma_{2} \wedge \Pi_{1}=\Pi_{2}\right) \wedge \\
& \left(\operatorname{Holds}\left(A\left(x_{1}, o_{1}, \Gamma_{1}, \Pi\right), z\right) \wedge \operatorname{Holds}\left(A\left(x_{2}, o_{2}, \Gamma_{2}, \Pi\right), z\right) \wedge \Pi \neq \emptyset\right. \\
& \left.\quad \supset x_{1}=x_{2} \wedge o_{1}=o_{2} \wedge \Gamma_{1}=\Gamma_{2}\right)
\end{aligned}
$$

In comparison with the uni-modal definition of the predicate Consistent, both versions implement the same concept, while their respective syntax differs slightly. Note that the uniqueness of identifiers regards individual hypotheses, while multiple hypotheses may be associated with the same situation. We expect all hypotheses to comply with the consistency criteria defined by the predicate Consistent.

$$
(\forall z)\left(\operatorname{KState}\left(S_{0}, z\right) \supset \text { Consistent }(z)\right)
$$

The Formula (5.13) requires that only those hypotheses existing in the initial situation have to be consistent. But we will show below that our symbol anchoring approach only generates consistent hypotheses. Given that the initial hypotheses are actually consistent, and given that the update of those only leads to consistent successive hypotheses, then all hypotheses are consistent (cf. Section 5.6.1). 


\subsection{Computing the Space of Hypotheses}

Next, we are going to see how our approach solves the first task of multi-modal symbol anchoring described in Section 5.1 on page 80, which is the task to determine the set of percepts that originate from the same physical object.

\subsubsection{Partitioning the Set of Percepts}

Let $\widehat{\Pi}_{s}$ denote the set of all percepts available in situation $s$ with

$$
\begin{gathered}
\widehat{\Pi}_{s}: \mathcal{P}(\text { PERCID }) \\
\widehat{\Pi}_{s}=\left\{\pi \mid(\exists) P\left(\pi, o, \Gamma, \pi_{R}, s\right)\right\}
\end{gathered}
$$

Hence, the cardinality of this set is determined by the function NoP such that

$$
(\forall s)\left(\exists ! k, \widehat{\Pi}_{s}\right)\left(N o P(s)=k \wedge\left|\widehat{\Pi}_{s}\right|=k\right)
$$

The set $\widehat{\Pi}_{s}$ should be considered a partition of subsets, where each subset includes only percepts that originate from the same physical object, and different subsets contain percepts originating from different physical objects. These subsets are the elements of the partition $\widehat{\Pi}_{s}$ and are called blocks. The concept of a mathematical partition fits well at this point due to its characteristics: First, no block is empty; second, the union of all blocks equals $\widehat{\Pi}_{s}$; and third, the individual blocks have to be pairwise disjoint.

We define the predicate PBlock, short for percept block, such that it determines a block of the partition of percepts of the given situation as follows.

$$
\text { PBlock : } \mathcal{P}(\text { PERCID }) \times \text { SIT }
$$

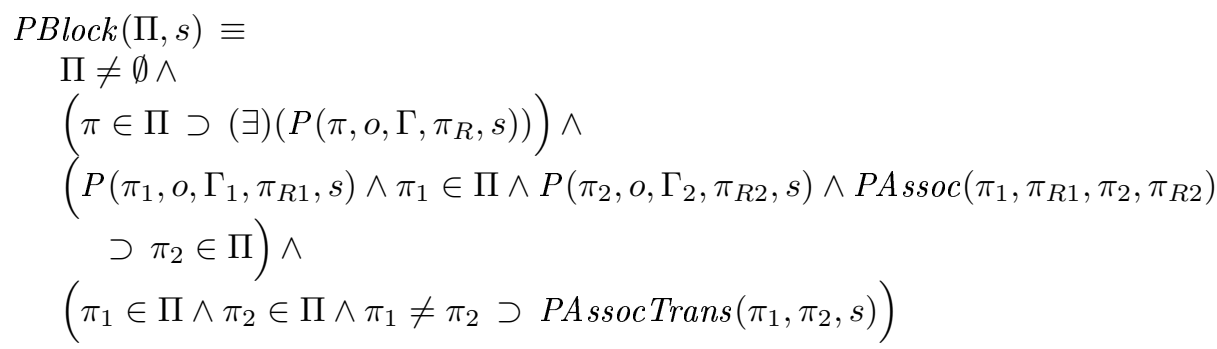

The predicate $P B \operatorname{lock}(\Pi, s)$ defines that those sets $\Pi$ are considered appropriate blocks of percepts if all three conjuncts of Formula (5.17) hold simultaneously in the given situation $s$. The first conjunct stipulates that an empty set does not constitute an appropriate block in accordance with the definition of a mathematical partition described above. This requirement is necessary in Formula (5.17), because the other two conjuncts are implications that otherwise would validate predicate $\operatorname{PBlock}(\Pi, s)$ with $\Pi=\emptyset$, too.

Being employed in Formula (5.17), the predicate PAssoc holds true if the given two percepts are directly related in terms of their reference percepts. Moreover, the predicate PAssocTrans holds true if the given two percepts are related transitively. Hence, the second conjunct of Formula (5.17) requires the transitive closure of the set $\Pi$ with respect to the relation defined by predicate PAssoc. This means for the second conjunct, if percept $\pi_{1}$ is element of a certain block $\Pi$ and $\pi_{1}$ is associated with percept $\pi_{2}$ in accordance with predicate PAssoc, then $\pi_{2}$ has to belong to the same block $\Pi$ as well. The third conjunct of Formula (5.17) verifies that no more percepts than those necessary for forming a complete block are part of a valid block $\Pi$. In particular, any two percepts of a valid block $\Pi$ have to be related to each other transitively. 
This way, the predicate PBlock defines that a valid block $\Pi$ must not contain several individual blocks of associated percepts, for instance. Note that the predicate PBlock also allows singleton sets to constitute valid blocks of percepts, if the percept of a singleton is not associated with any other current percept.

In summary, the predicate PBlock holds true for complete blocks of associated percepts whereof each percept of the same block originates from the same physical object. The macro $P A s s o c$ determines whether the given two percepts are directly related as follows.

$$
\text { PAssoc : PERCID } \times \text { PERCID } \times \text { PERCID } \times \text { PERCID }
$$

$$
\operatorname{PAssoc}\left(\pi_{1}, \pi_{R 1}, \pi_{2}, \pi_{R 2}\right) \stackrel{\text { def }}{=} \pi_{1} \neq \pi_{2} \wedge\left(\pi_{R 2}=\pi_{1} \vee \pi_{R 1}=\pi_{2} \vee \pi_{R 1}=\pi_{R 2} \wedge \pi_{R 1} \neq \perp\right)
$$

The Formula (5.18) defines the macro $P A \operatorname{ssoc}\left(\pi_{1}, \pi_{R 1}, \pi_{2}, \pi_{R 2}\right)$ to hold true for a given pair of percepts $\pi_{1}$ and $\pi_{2}$, if both percepts are distinct, and if one percept refers to the other or if both percepts share a common reference percept. The latter case of the macro PAssoc, where the two considered percepts share the same reference percept, is particularly important, because the reference percept may belong to a previous situation. Given that the object recognition and tracking system provides reference percepts as described in Section 5.2, PAssoc facilitates multimodal tracking by means of various sensors, as well as reacquiring a previously lost correspondence by means of any sensor available. Even though the common reference percept may have been measured in the current situation, possibly the tracking system reported the same percept for a while already, i.e., it may have been measured and registered previously, too. In general, the predicate PAssoc defines an irreflexive, symmetric and transitive relation between pairs of percepts of the same situation.

Being employed in the definition of predicate PBlock, the predicate PAssocTrans $\left(\pi_{1}, \pi_{2}, s\right)$ holds true if the given pair $\pi_{1}, \pi_{2}$ of percepts is related to each other transitively based on a direct relation between percepts determined by predicate PAssoc.

$$
\text { PAssocTrans : PERCID } \times \text { PERCID } \times \text { SIT }
$$

$$
\text { PAssocTransAux }: \text { PERCID } \times \text { PERCID } \times \mathcal{P}(\text { PERCID }) \times \text { SIT }
$$

$$
\operatorname{PAssocTrans}\left(\pi_{1}, \pi_{2}, s\right) \equiv \text { PAssocTransAux }\left(\pi_{1}, \pi_{2}, \emptyset, s\right)
$$

$$
\begin{aligned}
& \text { PAssocTransAux }\left(\pi_{1}, \pi_{2}, \Pi, s\right) \equiv \\
& (\exists)\left(P\left(\pi_{1}, o, \Gamma_{1}, \pi_{R 1}, s\right) \wedge P\left(\pi_{2}, o, \Gamma_{2}, \pi_{R 2}, s\right) \wedge \pi_{1} \neq \pi_{2} \wedge\right. \\
& P A \operatorname{ssoc}\left(\pi_{1}, \pi_{R 1}, \pi_{2}, \pi_{R 2}\right) \\
& \vee P\left(\pi_{3}, o, \Gamma_{3}, \pi_{R 3}, s\right) \wedge \pi_{3} \notin \Pi \wedge \\
& \text { PAssocTransAux } \left.\left.\left(\pi_{1}, \pi_{3}, \Pi \cup\left\{\pi_{2}\right\}, s\right) \wedge P A \operatorname{ssocTrans}\left(\pi_{2}, \pi_{3}, \Pi \cup\left\{\pi_{1}\right\} s\right)\right]\right)
\end{aligned}
$$

The definition of predicate PAssocTrans can be construed as a graph-based approach. Imagine a graph, the nodes of which each stand for one of all those percepts available in the considered situation, $\pi \in \widehat{\Pi}_{s}$, and where the relations $\left(\pi_{1}, \pi_{2}\right)$ determined by predicate PAssoc constitute the graph's undirected edges between the respective nodes. The transitive relation between two nodes holds, (a) if they are directly related to each other, or (b) if another node or several connected nodes can be found that connect the given nodes. The auxiliary predicate PAssocTransAux implements this concept as a disjunction within the square brackets of Formula (5.20). The predicate $P A s s o c$ verifies the direct relation between the given two percepts in case (a) of PAssocTransAux. In any case, PAssocTransAux requires $\pi_{1}$ and $\pi_{2}$ to denote valid and distinct percepts of the 
situation $s$ under consideration (cf. irreflexivity of PAssoc). An indirect, transitive relation between the given two percepts $\pi_{1}$ and $\pi_{2}$ holds in case (b) of PAssocTransAux, if there is another percept $\pi_{3}$ in the considered situation that is indirectly related to both $\pi_{1}$ and $\pi_{2}$. In terms of the above-mentioned graph interpretation case (b) holds if some node $\pi_{3}$ exists that connects both $\pi_{1}$ and $\pi_{2}$, while the connections between $\pi_{1}$ and $\pi_{3}$ as well as between $\pi_{2}$ and $\pi_{3}$ may in turn be indirect giving rise to further intermediate nodes.

Apparently, a recursive algorithm like this may consult more and more intermediate nodes or consider the same nodes again and again, such that it would never determine whether or not the original two percepts are actually related. For this purpose predicate PAssocTransAux carries the third parameter $\Pi \in \mathcal{P}$ (PERCID) representing the nodes which have been considered already and which must not be introduced as additional, intermediate nodes again. Accordingly, PAssocTransAux $\left(\pi_{1}, \pi_{2}, \emptyset, s\right)$ determines whether the percepts $\pi_{1}$ and $\pi_{2}$ are directly or indirectly related by any means, which stipulates predicate PAssocTrans in Formula (5.19) as a convenient abbreviation. The recursive invocations of predicate PAssocTransAux in the last line of its definition in the Formula (5.20) each amend the set $\Pi$ of already considered nodes by the original node of the current recursion level. Since PAssocTransAux regards only those percepts of the same situation $s$ as constituting valid, additional, intermediate nodes, which have not already been considered, no node will be examined twice within any subtree of recursions. Moreover, since only a finite number of percepts exist in any situation according to Section 5.3.1, only a finite number of recursions of predicate PAssocTrans are possible for any pair of percepts and any situation, due to PAssocTransAux strictly amending the set $\Pi$ of nodes already examined.

The predicate PAssocTransAux is sound and the recursive invocation is limited even though the restriction $\pi_{3} \neq \pi_{1} \wedge \pi_{3} \neq \pi_{2}$ is not explicitly stipulated in Formula (5.20)). However, predicate PAssocTransAux might choose $\pi_{3}$ such that $\pi_{3}=\pi_{1} \vee \pi_{3}=\pi_{2}$ and invoke two recursions, within which the condition $\pi_{1} \neq \pi_{2}$ of Formula (5.20) prevents any repeated use of the same percept. Since predicate PAssocTransAux implements an irreflexive, symmetric and transitive relation between two percepts, it fulfils the requirements regarding completeness.

\subsubsection{Proof of Correctness of Predicate PBlock}

Recall that $\widehat{\Pi}_{s}$ denotes the set of all percepts available in the situation $s$ (cf. Formula (5.14)). In this section we will show that the predicate PBlock holds for sets of percepts that are blocks of the partition $\widehat{\Pi}_{s}$. The proven correspondence with a partition will be useful later in this Chapter.

Lemma 5.1 The predicate PBlock defined in Formula (5.17) complies with the concept of a mathematical partition such that sets $\Pi$, which PBlock $(\Pi, s)$ holds for in the given situation $s$, are elements of a partition of the set $\widehat{\Pi}_{s}$.

Proof The definition of a mathematical partition requires that no subset of the partition is empty. This being an explicit conjunct in Formula (5.17), the predicate PBlock apparently only holds for non-empty sets.

For a block $\Pi$ to be valid in some situation $s$, the predicate $P B l o c k(\Pi, s)$ defines that each element $\pi \in \Pi$ must denote a valid percept of this situation. The implication

$$
(\forall s, \pi, \Pi)\left(P B \operatorname{lock}(\Pi, s) \wedge \pi \in \Pi \supset(\exists)\left(P\left(\pi, o, \Gamma, \pi_{R}, s\right)\right)\right.
$$

implements this requirement as part of Formula (5.17). Previously in Section 5.3.1 we have stipulated that there is a certain, limited number $k$ of percepts available in any situation $s$ as defined by function $N o P(s)=k$. This ensures that any valid block of percepts determined by predicate $P B l o c k$ includes a limited number of valid percepts in any situation. Note that this way predicate PBlock also prevents anything else than valid percepts from being elements of a valid percept block.

Given that the set of all available percepts $\widehat{\Pi}_{s}$ of a certain situation $s$ is limited according to Formulas (5.7) and (5.15), $\widehat{\Pi}_{s}$ is unique for situation $s$. The characteristics of percepts and the 
definition of predicate PBlock actually formulate a unique partition in any situation. Accordingly, each valid block of percepts constitutes a unique subset as an element of the partition of all percepts of a certain situation. We record this intermediate result in the following formula:

$$
\operatorname{PBlock}\left(\Pi_{1}, s\right) \wedge \operatorname{PBlock}\left(\Pi_{2}, s\right) \wedge \Pi_{1} \neq \Pi_{2} \supset \Pi_{1} \cap \Pi_{2}=\emptyset
$$

The predicate PBlock holds only for blocks of percepts that are transitively closed with respect to the associative relation between pairs of percepts determined by predicate PAssoc (cf. Section 5.4.1). Therefore, each valid percept identifier is an element of precisely one block of percepts of a certain situation, i. e., no percept belongs to multiple blocks of percepts of the same situation. Imagine to the contrary that the same percept, say $\pi^{*}$, would be part of multiple, different percept blocks. Then, due to the transitive closure of valid blocks of percepts, all percepts that have a transitive relation with percept $\pi^{*}$ would also have to be associated with those percept blocks which percept $\pi^{*}$ belongs to. But then all those percept blocks where $\pi^{*}$ belongs to would be equal with respect to set equality due to the characteristics of percepts. Hence, valid partitions as well as valid blocks of percepts are unique in any situation. Therefore, a universally quantified formula like $P B \operatorname{Block}(\Pi, s) \supset \Phi(\Pi, s)$ with blocks $\Pi$ in the range $\mathcal{P}$ (PERCID) according to Signature (5.16) specifies conditions $\Phi(\Pi, s)$ for any possible block of percepts. Since Formula (5.7) infers that each percept of a given situation $s$ is part of the partition $\widehat{\Pi}_{s}$ of this situation, a universally quantified formula like $\operatorname{PBlock}(\Pi, s) \supset \Phi(\Pi, s)$ considers each available percept of situation $s$. Altogether this shows that the predicate PBlock yields a mathematical partition of the set $\widehat{\Pi}_{s}$ of all available percepts of any considered situation. Conversely, it follows that each percept is a member of some block of percepts.

\subsubsection{Computing the Correspondences}

The next sections explicate how our approach solves the main task of multi-modal symbol anchoring as described in Section 5.1 on page 80, i. e., to determine all possible hypotheses of correspondences between object symbols and percepts representing objects of the robot's environment. Similar to our uni-modal approach described in Chapter 4, the multi-modal symbol anchoring employs the special epistemic called action AnchorM :

$$
\text { AnchorM : OBJCAT } \mapsto \text { ACTION }
$$

The knowledge update axiom of this action implements our multi-modal symbol anchoring axiom, similar to the uni-modal version. It computes the successive hypothesis space given the previous hypothesis space, the available percepts and the object category of interest in the considered situation. In other words, this axiom defines the rules of how to update the given knowledge state upon the execution of the epistemic symbol anchoring action yielding the knowledge state of the successor situation. We let the epistemic action AnchorM be applicable in any situation, i.e.

$$
(\forall)(\operatorname{Poss}(\operatorname{AnchorM}(o), s))
$$

In contrast to the uni-modal symbol anchoring axiom (cf. Formula (4.15) on page 50), the multimodal symbol anchoring axiom does not implement a schematic axiom. Instead of enumerating each individual percept as in the uni-modal axiom, the multi-modal axiom universally quantifies all possible blocks of percepts as follows. 


$$
\begin{aligned}
& \operatorname{Poss}(\operatorname{AnchorM}(o), s) \supset\left(\exists s^{\prime}\right)\left(s^{\prime}=\operatorname{Do}(\operatorname{Anchor} M(o), s) \wedge\right. \\
& \left(\forall z^{\prime}\right)\left(K \operatorname{KState}\left(s^{\prime}, z^{\prime}\right) \equiv\left(\exists z, \vartheta^{-}, \vartheta^{+}, \vartheta\right)(\text { KState }(s, z) \wedge\right. \\
& {\left[\operatorname { P B l o c k } ( \Pi _ { P } , s ) \supset ( \exists x , f ^ { - } , f ^ { + } ) \left(\operatorname{Holds}\left(C\left(x, \Pi_{P}, f^{-}, f^{+}\right), \vartheta\right) \wedge\right.\right.} \\
& \left.\left.\left[\Delta_{T}\left(x, \Pi_{P}, f^{-}, f^{+}, s, z\right) \vee \Delta_{F}\left(x, \Pi_{P}, f^{-}, f^{+}, s, z\right) \vee \Delta_{N}\left(x, \Pi_{P}, f^{-}, f^{+}, s, z\right)\right]\right)\right] \wedge \\
& {\left[\operatorname{Holds}(f, \vartheta) \supset\left(\exists x, \Pi_{P}, f^{-}, f^{+}\right)\left(f=C\left(x, \Pi_{P}, f^{-}, f^{+}\right) \wedge \operatorname{PBlock}\left(\Pi_{P}, s\right)\right)\right] \wedge} \\
& \operatorname{Holds}\left(C\left(x_{1}, \Pi_{P}, f_{1}^{-}, f_{1}^{+}\right), \vartheta\right) \wedge \operatorname{Holds}\left(C\left(x_{2}, \Pi_{P}, f_{2}^{-}, f_{2}^{+}\right), \vartheta\right) \\
& \left.\supset x_{1}=x_{2} \wedge f_{1}^{-}=f_{2}^{-} \wedge f_{1}^{+}=f_{2}^{+}\right] \wedge \\
& {\left[\operatorname{Holds}\left(C\left(x, \Pi_{P 1}, f_{1}^{-}, f_{1}^{+}\right), \vartheta\right) \wedge \operatorname{Holds}\left(C\left(x, \Pi_{P 2}, f_{2}^{-}, f_{2}^{+}\right), \vartheta\right)\right.} \\
& \left.\supset \Pi_{P 1}=\Pi_{P 2} \wedge f_{1}^{-}=f_{2}^{-} \wedge f_{1}^{+}=f_{2}^{+}\right] \wedge \\
& {\left[\operatorname{Holds}\left(f^{+}, \vartheta^{+}\right) \equiv\left(\exists x, f^{-}, \Pi_{P}\right)\left(\operatorname{Holds}\left(C\left(x, \Pi_{P}, f^{-}, f^{+}\right), \vartheta\right)\right)\right] \wedge} \\
& {\left[\operatorname{Holds}\left(f^{-}, \vartheta^{-}\right) \equiv\left(\exists x, f^{+}, \Pi_{P}\right)\left(\operatorname{Holds}\left(C\left(x, \Pi_{P}, f^{-}, f^{+}\right), \vartheta\right) \wedge f^{-} \neq \emptyset\right)\right] \wedge} \\
& \left.\left.\left.z^{\prime}=z-\vartheta^{-}+\vartheta^{+}\right)\right)\right)
\end{aligned}
$$

Let's structure this symbol anchoring axiom in order to enhance its readability abstracting from the conjuncts in the middle by replacing them with the formula $\Phi\left(\vartheta^{-}, \vartheta^{+}, \vartheta, s, z\right)$, forming the following, equivalent axiom.

$$
\begin{aligned}
& \operatorname{Poss}(\operatorname{AnchorM}(o), s) \supset\left(\exists s^{\prime}\right)\left(s^{\prime}=\operatorname{Do}(\operatorname{AnchorM}(o), s) \wedge\right. \\
& \left(\forall z^{\prime}\right)\left(\text { KState }\left(s^{\prime}, z^{\prime}\right) \equiv\left(\exists z, \vartheta^{-}, \vartheta^{+}, \vartheta\right)(\text { KState }(s, z) \wedge\right. \\
& \Phi\left(\vartheta^{-}, \vartheta^{+}, \vartheta, s, z\right) \wedge \\
& \left.\left.\left.z^{\prime}=z-\vartheta^{-}+\vartheta^{+}\right)\right)\right)
\end{aligned}
$$

The abstract knowledge update Axiom (5.24) can be interpreted as follows. If the symbol anchoring action AnchorM was applicable in situation $s$, then the knowledge state of the successor situation $s^{\prime}$ is determined from the knowledge state of situation $s$ by subtracting the state term $\vartheta^{-}$ and adding the state term $\vartheta^{+}$which both are governed by the formula $\Phi\left(\vartheta^{-}, \vartheta^{+}, \vartheta, s, z\right)$. Besides determining possible values of the terms $\vartheta^{-}, \vartheta^{+}$and $\vartheta$, the formula $\Phi\left(\vartheta^{-}, \vartheta^{+}, \vartheta, s, z\right)$ may also restrict the existence of possible successor states altogether. Note that multiple possible states $z$ may exist in the original situation $s$ in general, and for each of those states the axiom may give rise to zero to $n$ successor states $z^{\prime}$. A discussion on the complexity of the symbol anchoring Axiom (5.23), including the number $n$, will be provided below (cf. Section 5.4.7). In abstract terms, the symbol anchoring Axiom (5.24) specifies that for any successive hypothesis $z^{\prime}$ to exist, some hypothesis $z$ must hold in the original situation $s$ and formula $\Phi\left(\vartheta^{-}, \vartheta^{+}, \vartheta, s, z\right)$ verifies some relation between $z$ and $z^{\prime}$.

As an overview, our symbol anchoring Axiom (5.23) can be considered to perform three tasks:

1. Given the current percepts, valid correspondences between these percepts and object identifiers are determined. The resulting new correspondences are gathered in a temporary state term in order to facilitate the exposure of the new, whole hypothesis to certain consistency constraints.

2. Considering the new, suggested hypothesis altogether, certain consistency constraints are applied, such that inconsistent candidate hypotheses are neglected.

3. After some data reorganization the new hypothesis is obtained from updating the considered original one in accordance with the changes determined in step 1. 
Let's have a close look at the Axiom (5.23). The crucial computational part of the symbol anchoring transaction is performed by the second implication beginning with $\operatorname{PBlock}\left(\Pi_{P}, s\right) \supset \ldots$ inside the axiom. Being a conjunct of Axiom (5.23) with universal quantification of $\Pi_{P}$, this implication requires for all valid blocks of percepts that at least one instance of one of the three functionalities (macros $\Delta_{T}, \Delta_{F}, \Delta_{N}$ ) holds true. We will see below, that the definitions of these functionalities guarantee that at least one of them is actually satisfiable, such that the existentially quantified disjunction of the three functionalities is guaranteed to be satisfiable in the end. The auxiliary fluent $C$ is used to record the chosen instances of variables satisfying the disjunction of functionalities, where

$$
C: \text { PERCID } \cup \mathcal{X} \times \mathcal{P}(\text { PERCID }) \times \text { FLUENT } \times \text { FLUENT } \mapsto \text { FLUENT }
$$

The conjunct $\operatorname{Holds}\left(C\left(x, \Pi_{P}, f^{-}, f^{+}\right), \vartheta\right)$ stores grounded instances of fluent $C$ in the state term $\vartheta$. This term is always satisfiable per se for the existentially quantified, incomplete state variable $\vartheta$. The existential quantification and scope of the state terms $\vartheta, \vartheta^{-}$and $\vartheta^{+}$are well chosen such that all conditions of Axiom (5.23) have to hold true for a single instance of these state terms, but for all blocks of percepts simultaneously. Hence, for each valid block of percepts an instance of the auxiliary fluent $C$ is accumulated inside the state $\vartheta$. The fluent $C$ and the state term $\vartheta$ serve the purpose to store the correspondences found temporarily, and to impose certain restrictions on $\vartheta$ in order to filter just the valid correspondences.

In case the premise $\operatorname{PBlock}\left(\Pi_{P}, s\right)$ of the second conjunct of Axiom (5.23)-which itself is an implication - is false, i. e., in case an invalid block of percepts $\Pi_{P}$ is considered, the inference holds true irrespective of the values of variables involved. Therefore, the conjunct which begins with $\operatorname{Holds}(f, \vartheta) \supset \ldots$ is necessary in order to restrict the state term $\vartheta$ to include only fluents $C$ for valid blocks of percepts. More precisely, the first conjunct of the consequence of this implication requires that only instances of fluent $C$ may be part of $\vartheta$, while the second conjunct requires that blocks of percepts are valid with respect to predicate $P B l o c k$. Note that for each valid block of percepts $\Pi_{P}$ the first implication of Axiom (5.23) (beginning with $P B l o c k\left(\Pi_{P}, s\right) \supset \ldots$ ) ensures that an applicable functionality determines grounded instances of the fluent $C$ representing valid, new correspondences, that enter the state term $\vartheta$. The first two implications of Axiom (5.23) in combination turn the incomplete state variable $\vartheta$ into a well-defined, finite state, because the set of all available percepts $\widehat{\Pi}_{s}$ is finite, the partition of $\widehat{\Pi}_{s}$ is unique in accordance with predicate PBlock, and because only fluents $C$ are possible members of $\vartheta$.

The correspondences recorded in form of fluent $C$ in the state $\vartheta$ are subject to further constraints before they are considered as one of the new correspondences. The third and fourth sub-formula of Axiom (5.23) enclosed in large, squared brackets constrain the state term $\vartheta$ to comprise correspondence fluents $C$, which are unique with respect to the block of percepts at hand (second parameter), and also with respect to the the object identifier (first parameter). Both aspects of uniqueness of correspondences are crucial in order to ensure and to maintain the consistency of all correspondences.

Note that the above-mentioned constraints on state $\vartheta$ have the effect that the Axiom (5.23) chooses exactly one correspondence among the valid correspondences non-deterministically for any successor hypothesis $z^{\prime}$ and block of percepts $\Pi_{P}$. However, as the disjunction of functionalities may yield multiple valid correspondences for the same block of percepts $\Pi_{P}$, additional alternative hypotheses $z^{\prime}$ may become introduced this way.

As you will see below, each of the three macros $\Delta_{T}, \Delta_{F}$ and $\Delta_{N}$ implementing the three symbol anchoring functionalities prepare two fluents for each correspondence to be updated by the Axiom (5.23): First, the anchor fluent $f^{-}$represents the current correspondence and is to be subtracted from the hypothesis representation $z$. Second, the anchor fluent $f^{+}$represents the updated correspondence and has to be added to the hypothesis representation $z^{\prime}$. In other words, all information about retracting the old anchor and replacing it with the new anchor is included in the fluent $C$. The last two conjuncts of Axiom (5.23) enclosed in large, squared brackets extract the components $f^{-}$and $f^{+}$from all fluents $C$ of $\vartheta$ in order to collect them in the state terms $\vartheta^{-}$and $\vartheta^{+}$, respectively. Eventually, a successor hypothesis is constructed by subtracting 
the aggregate state $\vartheta^{-}$from and adding $\vartheta^{+}$to the original hypothesis considered, as can be seen in the last conjunct of the symbol anchoring Axiom (5.23).

Returning to the conjunct with the first implication beginning with $P B \operatorname{Block}\left(\Pi_{P}, s\right) \supset \ldots$, recall that we recognized above that the conclusion is always satisfiable given valid blocks of percepts $\Pi_{P}$. The more important result is that this way this implication implements our fundamental concept described in Section 3.4: Each percept has to be assigned an object symbol. As multiple percepts may refer to the same physical object in the multi-modal case, the corresponding concept here is to assign each block of associated percepts an object symbol.

Even in the case where no percept is available, the symbol anchoring Axiom (5.23) is correct. In this case, the state terms $\vartheta, \vartheta^{-}$and $\vartheta^{+}$equal empty states such that the state update $z^{\prime}=z-\vartheta^{-}+\vartheta^{+}$simply becomes $z^{\prime}=z$ and the successor hypothesis $z^{\prime}$ is determined to be the exact copy of the original hypothesis $z$. This reflects the expected behaviour, i. e., if no new perceptual information is available, the symbol anchoring system does not alter the current world model.

\subsubsection{Tracking an Object}

Similar to our uni-modal approach presented in Chapter 4, tracking an object and updating its anchor representation is one of three main symbol anchoring functionalities of our approach. Each of the three functionalities applies in a certain, distinct configuration of object symbol, anchor and percept under consideration. The task of the functionality "Track" is to update the representation of a correspondence, given one or more new percepts that have been tracked by the object recognition system. In accordance with our assumptions on the interface between object recognition and symbol anchoring described in Section 5.2, the recognition system is asked to notify the successful tracking of an object of the environment by means of simply reusing the same percept identifier or by referring to a previous percept. For this purpose we added a parameter to the percept predicate, which holds the reference percept. Our multi-modal approach allows multiple percepts to represent the measured object (cf. Section 5.3.1). Given our assumptions of Section 3.11 on the object recognition system, the symbol anchoring system may consider all those new percepts that were reported to refer to the same, previous percept as resulting from the same object of the environment. In this case, functionality Track is applicable and basically fuses all available perceptual information in the anchor representation. The macro $\Delta_{T}$ implements the multi-modal functionality Track as follows.

$$
\begin{aligned}
& \Delta_{T}: \mathcal{X} \cup \text { PERCID } \times \mathcal{P}(\text { PERCID }) \times \text { FLUENT } \times \text { FLUENT } \times \text { SIT } \times \text { STATE } \\
& \Delta_{T}\left(x, \Pi_{P}, f, f^{\prime}, s, z\right) \stackrel{\text { def }}{=} \\
& \left(\exists o, \Gamma_{A}, \Pi_{A}, \Gamma^{\prime}, \Pi^{\prime}\right)\left(\text { Holds }(f, z) \wedge f=A\left(x, o, \Gamma_{A}, \Pi_{A}\right) \wedge f^{\prime}=A\left(x, o, \Gamma^{\prime}, \Pi^{\prime}\right) \wedge\right. \\
& {\left[\pi_{P} \in \Pi_{P}\right.} \\
& \left.\quad \supset\left(\exists \Gamma_{P}, \pi_{R}\right)\left(P\left(\pi_{P}, o, \Gamma_{P}, \pi_{R}, s\right) \wedge P \operatorname{Pnown}\left(\Pi_{A}, \pi_{P}, \pi_{R}\right) \wedge \operatorname{Match}\left(x, \Gamma_{P}, z\right)\right)\right] \wedge \\
& \text { AnchorUpdate } \left.\left(o, \Gamma_{A}, \Pi_{A}, \Pi_{P}, \Gamma^{\prime}, \Pi^{\prime}, s\right)\right)
\end{aligned}
$$

The multi-modal symbol anchoring Axiom (5.23) employs the macro $\Delta_{T}$ with the existentially quantified variables $x, f$, and $f^{\prime}$ and with ground instances of $\Pi_{P}, s$ and $z$. This way macro $\Delta_{T}$ determines the existing correspondence, which the new block $\Pi_{P}$ of percepts refers to, the current anchor fluent $f$ and the updated anchor fluent $f^{\prime}$, if Track applies to $\Pi_{P}$. In case the functionality Track actually applies to the given block of percepts, situation and hypothesis, the variables $x$, $f$ and $f^{\prime}$ will be instantiated deterministically, i.e., there is exactly one instance of each of these variables that satisfies the Formula (5.25). Finally, the Axiom (5.23) replaces the anchor fluent $f$ of the considered correspondence and hypothesis with the new anchor $f^{\prime}$ in the successor hypothesis. 
In detail, the macro $\Delta_{T}\left(x, \Pi_{P}, f, f^{\prime}, s, z\right)$ implementing the functionality Track requires the fluent $f$ to be an anchor fluent of object category o existing in the given hypothesis $z$. The updated anchor fluent $f^{\prime}$ continues to refer to object $x$ of category $o$. Likewise, all percepts of the considered block $\Pi_{P}$ have to belong to category $o$. The macro $\Delta_{T}$ requires for the anchor of object $x$ and for each percept $\pi_{P}$ of the block $\Pi_{P}$ that the percepts $\pi_{P}$ refer to some percepts recorded previously in the anchor of $x$ by means of predicate PKnown. Also, the new information included in each percept $\pi_{P} \in \Pi_{P}$ has to comply with the best perceptual estimate gathered so far in the anchor of object $x$, as well as with all available symbolic information about this object. The predicate Match implements this requirement.

The predicate PKnown is used in Formula (5.25) in order to check whether the given percept is being tracked and belongs to the considered anchor. In accordance with the assumptions defining the interface between our symbol anchoring system and the object recognition explicated in Sections 3.11 and 5.2, it is sufficient for predicate PKnown to check whether the primary or the reference percept identifier has been recorded in the given anchor already. Note that the predicate PKnown facilitates multi-modal tracking of an object this way. Thus the tracking system may switch to other sensor devices while tracking the same object continuously, because in this case a different, new percept $\pi_{P}$ is reported that refers to a previous, already known percept. This simple mechanism only works if all new percepts are recorded in the anchor, which our approach actually implements inside the predicate AnchorUpdate. We define the predicate PKnown as follows.

$$
\begin{gathered}
\text { PKnown : } \mathcal{P}(\mathrm{PERCID}) \times \mathrm{PERCID} \times \mathrm{PERCID} \cup\{\perp\} \\
P \text { Known }\left(\Pi_{A}, \pi_{P}, \pi_{R}\right) \equiv \pi_{P} \in \Pi_{A} \vee \pi_{R} \in \Pi_{A}
\end{gathered}
$$

In an alternative approach to predicate $P K n o w n\left(\Pi_{A}, \pi_{P}, \pi_{R}\right)$, one could test for a transitive relation between the primary percept identifier $\pi_{P}$ and some recorded identifier of the set $\Pi_{A}$ of previously corresponding percepts, which holds via a chain of intermediate reference percepts. However, given the requirements on the tracking system described above that seem to be not too strong to cause practical difficulties, a simple verification of a direct relation as implemented in Formula (5.26) serves the purpose sufficiently.

The definition of the functionality Track by means of macro $\Delta_{T}$ in Formula (5.25) employs the predicate Match. Basically, the task of this predicate is to verify whether all new perceptual information on the considered object agrees with all the information associated with the corresponding object symbol and anchor. In particular, the predicate Match checks whether the new percept complies with first, the best perceptual estimate representing previous measurements gathered so far, and second, with the symbolic knowledge. Both checks are limited to static attributes only for reasons explained in Section 4.2.2. For short, only information about static perceptual attributes allows our approach to identify inconsistent hypotheses definitely.

$$
\text { Match }: \mathcal{X} \cup \text { PERCID } \times \text { PERCSIG } \times \text { STATE }
$$

$$
\begin{gathered}
\operatorname{Match}\left(x, \Gamma_{P}, z\right) \equiv\left(\exists o, \Gamma_{A}, \Pi_{A}\right)\left(\operatorname{Holds}\left(A\left(x, o, \Gamma_{A}, \Pi_{A}\right), z\right) \wedge\right. \\
(\forall)\left(\left(\gamma_{P} \in \Gamma_{P} \wedge \gamma_{P}=\left(a, i_{P}\right) \wedge \gamma_{A} \in \Gamma_{A} \wedge \gamma_{A}=\left(a, i_{A}\right) \wedge \operatorname{AttrTypeStatic}(o, a)\right.\right. \\
\left.\supset \text { Intersect }\left(i_{P}, i_{A}\right)\right) \wedge \\
\left(\gamma_{P} \in \Gamma_{P} \wedge \gamma_{P}=\left(a, i_{P}\right) \wedge \operatorname{Holds}(\operatorname{Prop}(x, a, v), z) \wedge \operatorname{AttrTypeStatic}(o, a)\right. \\
\left.\left.\left.\supset \text { GroundingInt }\left(o, a, v, i_{P}\right)\right)\right)\right)
\end{gathered}
$$

This definition of predicate Match differs only slightly from the uni-modal version in Formula (4.20), namely only with respect to the syntax of the anchor fluent $A$. Since the predicate Match regards a single percept only, a similar definition may be used in both our uni-modal and multi-modal 
versions. The reader is referred to the detailed description of the uni-modal version of predicate Match in Section 4.2.2, which applies likewise to the multi-modal case.

Eventually, the functionality Track performs the update of a certain anchor for the considered block of percepts by means of predicate AnchorUpdate in Formula (5.25). Since not all components of the anchor change, AnchorUpdate takes just those as input that are to be updated besides some contextual information like the object category and situation under consideration. The Formula (5.28) performs the update as follows.

AnchorUpdate $:$ OBJCAT $\times$ PERCSIG $\times \mathcal{P}($ PERCID $) \times \mathcal{P}($ PERCID $) \times$ PERCSIG $\times \mathcal{P}($ PERCID $) \times$ SIT

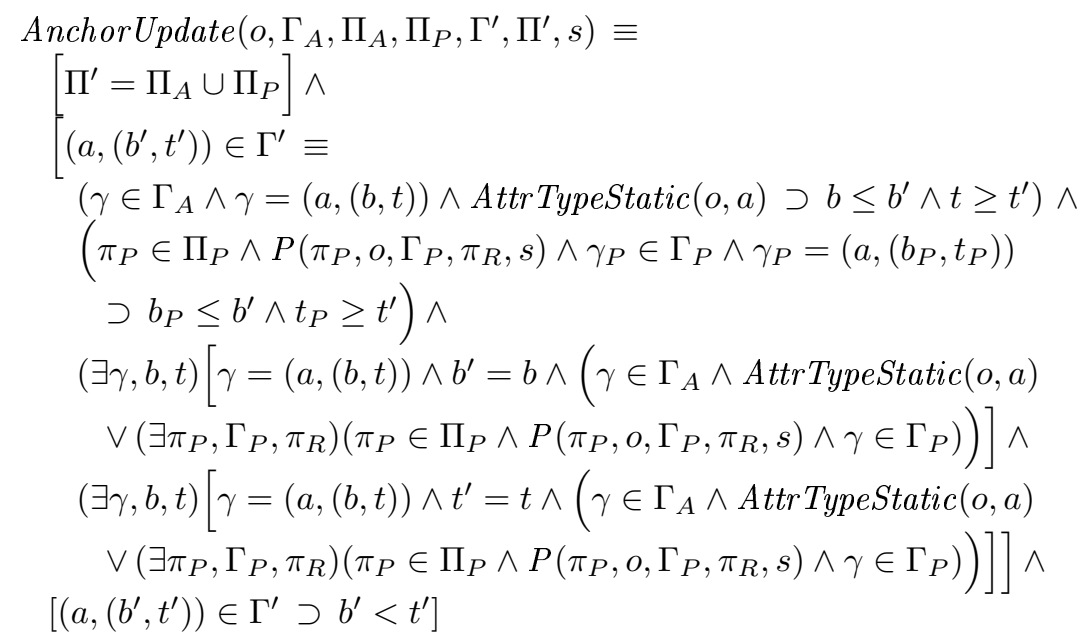

In accordance with our interface definition between symbol anchoring and object recognition systems described in Section 5.2, we assume that the recognition and tracking system reports an object as being tracked successfully by either reusing the same percept identifier or by referring to a previous one. Therefore, upon every symbol anchoring transaction all new percept identifiers have to be added to the set of previously corresponding percepts $\Pi_{A}$ of the anchor. The first conjunct of Formula (5.28) accomplishes this step.

In Section 5.2 above we required that the perceptual values of individual perceptual attributes of objects in the environment of the cognitive robot may be represented as a uni-modal distribution of measured values. Our approach in particular uses a straight interval of values wherein the actually measured perceptual values fall. Recall our concept of maintaining the best perceptual estimate inside the anchor of a correspondence, which records the smallest interval of perceptual values of each perceptual attribute experienced by the robot so far about the considered object (cf. Section 4.1.2). In general, new measurements of dynamic perceptual attributes replace the previously recorded perceptual estimate, because we consider old measurements as out-of-date records. Conversely, new measurements of static perceptual attributes refine the current estimate by fusing the two in a single, minimal interval of perceptual values. The latter method of fusing previous estimates and new measurements is justified by the fact that the validity of perceptual values of static attributes persists over time.

The second, complex conjunct of the definition of predicate AnchorUpdate in Formula (5.28) determines the new interval representation $\left(b^{\prime}, t^{\prime}\right)$ for each perceptual attribute $a$ that the cognitive robot knows of. The updated, best perceptual estimate $\Gamma^{\prime}$ includes the new interval $\left(b^{\prime}, t^{\prime}\right)$ if and only if each of the following items holds for each perceptual attribute $a$ :

- The updated interval $\left(b^{\prime}, t^{\prime}\right)$ is smaller than or equal to the current interval $(b, t)$ of the best perceptual estimate $\Gamma$ for static perceptual attributes. This way, previous information persists if the new percepts do not contain more detailed measurements. 
- The next conjunct beginning with $\pi_{P} \in \Pi_{P} \wedge$.. in Formula (5.28) performs sensor fusion among all available, associated percepts of the considered block $\Pi_{P}$. Regardless of which sensor was employed for measuring the percept, the most precise interval of perceptual values determines the new, best perceptual estimate. In particular, the new interval is constrained by the most precise percept such that $\left(b^{\prime}, t^{\prime}\right)$ becomes at least as precise as the previous estimate $(b, t)$.

- Having constrained the new interval $\left(b^{\prime}, t^{\prime}\right)$ for a certain attribute $a$ above, this also infers that $\left(b^{\prime}, t^{\prime}\right)$ may take on arbitrary values within the limiting perceptual values. Therefore, the last two conjuncts of the right hand side of the equivalence of Formula (5.28) that begin with $(\exists \gamma, b, t)$ specify that the new interval limits $b^{\prime}$ and $t^{\prime}$ each have to be equal to either the respective limit of a static perceptual attribute of the current best estimate $\gamma \in \Gamma_{A}$, or to the limit of the perceptual interval of some percept of the considered block $\Pi_{P}$.

- The final conjunct of the definition of predicate AnchorUpdate in Formula (5.28) requires that all associated percepts of the considered block $\Pi_{P}$ sustain the assumption of a unimodal distribution of perceptual values consistently, such that the most constrained, new perceptual estimate bears meaningful values in accordance with our assumptions described in Sections 3.5 and 3.11. Note that this requirement goes beyond the Constraint (5.5) regarding individual percepts, because its scope ranges over a full block of percepts. Even though two percepts may fulfil the Formula (5.5), they may include disjoint intervals of perceptual values of the same attribute, which the definition of functionality Track prevents appropriately.

Note that both the bottom and top limits of the intervals of the new percepts and of the perceptual estimate are considered independently, such that each one may refine the limit of the new estimate individually. This way, the predicate AnchorUpdate makes full use of all the available perceptual information.

In summary, the predicate AnchorUpdate performs sensor fusion of all new percepts of the considered block. Thus it implements the second task of multi-modal symbol anchoring described in Section 5.1 on page 80 . The so-gained fused aggregate intervals of dynamic attributes simply replace the previous estimate inside the anchor. The predicate AnchorUpdate fuses the aggregate intervals of static attributes with the previous estimate forming the new perceptual estimate. The Formula (5.28) is designed to even take such situations into account where no percept is available, where a block comprises just a single percept, where a perceptual estimate is or is not existent inside the anchor for individual perceptual attributes, and where percepts do or do not provide information on individual perceptual attributes. In other words, the versatile predicate AnchorUpdate adapts to any possible state of the representation.

Altogether, the functionality Track defined in form of macro $\Delta_{T}$ in Formula (5.25) determines first, whether Track is applicable, and determines second, the outdated and new anchor fluents appropriately, if applicable. We will see and describe below in detail, that the applicability conditions of functionalities Track and Find as well as of Track and New are pairwise disjoint. In other words, if functionality Track is applicable in the considered situation for the given block of percepts, it is the only functionality being applicable and no alternative hypothesis of a correspondence with another object symbol is allowed.

\subsubsection{Finding and Reacquiring a Correspondence}

For the same reasons as explained in Section 4.2.3, symbol anchoring demands further functionalities besides tracking an object, e.g., in case the tracking of an object fails. Therefore, the disjunction of functionalities in our symbol anchoring Axiom (5.23) also includes the functionalities "Find" and "New" besides "Track". The conceptual task of functionality Find is actually twofold, which we realized in a single formula. In both cases, the task is to identify an appropriate block of percepts that never corresponded before with the considered, matching object symbol. This block of percepts must not be involved in any correspondence being tracked. On the one hand, the multi-modal functionality Find applies if the block of percepts constitutes a potential 
candidate for the correspondence with an object symbol that has not yet been anchored before. On the other hand, a new block of percepts may represent an object of the environment which the recognition system failed to track previously, and of which it obtained new percepts afterwards that could not be associated with some previous percept of the same object. In other words, the purpose of this symbol anchoring functionality is to find an appropriate, new correspondence, and to reacquire a previously existing correspondence.

Accordingly, the applicability of functionality Find includes first, that the considered anchor must not correspond to any percept already. Otherwise, the functionality Track would apply instead as we pointed out in Section 5.4.4 above. Second, the block of percepts must not already correspond with another object symbol. The contrary case covers the functionality Track, too. Apparently, these two conditions bring about the intended effect that the functionalities Track and Find do not apply in the same configuration of block of percepts, object symbol and hypothesis. We define the functionality Find in form of macro $\Delta_{F}$ as follows:

$$
\begin{aligned}
& \Delta_{F}: \mathcal{X} \cup \text { PERCID } \times \mathcal{P}(\text { PERCID }) \times \text { FLUENT } \times \text { FLUENT } \times \text { SIT } \times \text { STATE } \\
& \Delta_{F}\left(x, \Pi_{P}, f, f^{\prime}, s, z\right) \stackrel{\text { def }}{=} \\
& \left(\exists o, \Gamma_{A}, \Pi_{A}, \Gamma^{\prime}, \Pi^{\prime}\right)\left(\text { Holds }(f, z) \wedge f=A\left(x, o, \Gamma_{A}, \Pi_{A}\right) \wedge f^{\prime}=A\left(x, o, \Gamma^{\prime}, \Pi^{\prime}\right) \wedge\right. \\
& \neg\left(\exists \pi_{1}, o_{1}, \Gamma_{1}, \pi_{R 1}\right)\left(P\left(\pi_{1}, o_{1}, \Gamma_{1}, \pi_{R 1}, s\right) \wedge P \operatorname{Pnown}\left(\Pi_{A}, \pi_{1}, \pi_{R 1}\right)\right) \wedge \\
& {\left[\pi _ { P } \in \Pi _ { P } \supset ( \exists \Gamma _ { P } , \pi _ { R } ) \left(P\left(\pi_{P}, o, \Gamma_{P}, \pi_{R}, s\right) \wedge \operatorname{Match}\left(x, \Gamma_{P}, z\right) \wedge\right.\right.} \\
& \left.\neg\left(\exists y, o_{y}, \Gamma_{y}, \Pi_{y}\right)\left(H o l d s\left(A\left(y, o_{y}, \Gamma_{y}, \Pi_{y}\right), z\right) \wedge P \operatorname{Pnown}\left(\Pi_{y}, \pi_{P}, \pi_{R}\right)\right)\right) \wedge \\
& \text { AnchorUpdate } \left.\left(o, \Gamma_{A}, \Pi_{A}, \Pi_{P}, \Gamma^{\prime}, \Pi^{\prime}, s\right)\right)
\end{aligned}
$$

The macro $\Delta_{F}\left(x, \Pi_{P}, f, f^{\prime}, s, z\right)$ is employed in the symbol anchoring Axiom (5.23) with the existentially quantified variables $x, f$, and $f^{\prime}$, while $\Pi_{P}, s$ and $z$ are ground. This way, the macro $\Delta_{F}$ is used to determine an appropriate object symbol $x$ for the given block of percepts $\Pi_{P}$ to be anchored for the given situation $s$ and hypothesis $z$. At the same time, the fluents $f$ and $f^{\prime}$ are prepared for being removed from and added to the hypothesis representation $z$, respectively. In general, the functionality Find may be applicable to multiple, possible object symbols $x$ given a certain block of percepts $\Pi_{P}$, depending on whether the object's anchor fulfils the requirements specified by macro $\Delta_{F}$. Each such alternative object symbol gives rise to an alternative potential hypothesis.

The macro $\Delta_{F}$ can be interpreted as follows. In order to satisfy $\Delta_{F}\left(x, \Pi_{P}, f, f^{\prime}, s, z\right)$, some anchor $f$ has to exist in hypothesis $z$, which does not correspond with any percept in situation $s$. Furthermore, the implication beginning with $\pi_{P} \in \Pi_{P} \supset \ldots$ requires first, that each percept of the given block $\Pi_{P}$ has to sustain the currently available perceptual and symbolic information about object $x$ consistently according to predicate Match, and second, that no percept $\pi_{P} \in \Pi_{P}$ may correspond with any anchor in hypothesis $z$. The macro $\Delta_{F}$ restricts the potentially matching objects to the same category as was reported by the recognition system. Finally, the predicate AnchorUpdate fuses all new perceptual information into the updated, best perceptual estimate $\Gamma^{\prime}$ and records those percept identifiers associated with the object $x$ in $\Pi^{\prime}$. The reader is referred to the Section 5.4.4 for the definition and description of the predicates Match and AnchorUpdate.

In summary, the macro $\Delta_{F}$ determines whether the functionality Find is applicable to the given block of percepts, and if so it suggests a potential object and prepares the fluents for the update of the considered hypothesis. Note that the multi-modal functionality Find takes all percepts available for a certain object into account at once, regardless which sensor device they stem from.

\subsubsection{Introducing a New Object}

Being the third functionality employed in the symbol anchoring Axiom (5.23), the functionality "New" accounts for the possibility of any new block of percepts, that is not being tracked success- 
fully by the recognition system, to stand for a yet completely unknown object. Accordingly, New is always applicable to blocks of percepts, which the functionality Track does not apply to. Likewise does the functionality Find in relation to Track. We argued in Section 3.10 for maintaining each potential hypothesis of correspondences. Since the possibility that a new block of percepts may not refer to a known object cannot simply be excluded, our approach introduces an alternative hypothesis for this case. We used the same rationale for the uni-modal version of functionality New in Section 4.2.4.

A cognitive robot acting and reasoning in a dynamic, partially unknown environment is likely to experience yet unknown objects. In order to be able to act on the objects and to reason about them, the robot has to learn about their features and to perform symbol anchoring also for those unknown objects. These two interpretations sustain the definition of the multi-modal symbol anchoring functionality New by means of macro $\Delta_{N}$ defined as follows.

$$
\Delta_{N}: \text { PERCID } \times \mathcal{P}(\text { PERCID }) \times \text { FLUENT } \times \text { FLUENT } \times \text { SIT } \times \text { STATE }
$$

$$
\begin{aligned}
& \Delta_{N}\left(x, \Pi_{P}, f, f^{\prime}, s, z\right) \stackrel{\text { def }}{=} \\
& f=\emptyset \wedge\left(\exists o, \Gamma^{\prime}\right)\left(f^{\prime}=A\left(x, o, \Gamma^{\prime}, \Pi_{P}\right) \wedge\right. \\
& \quad\left[\pi_{P} \in \Pi_{P} \supset x \geq \pi_{P} \wedge\left(\exists \Gamma_{P}, \pi_{R}\right)\left(P\left(\pi_{P}, o, \Gamma_{P}, \pi_{R}, s\right) \wedge\right.\right. \\
& \left.\left.\quad \neg\left(\exists y, o_{y}, \Gamma_{y}, \Pi_{y}\right)\left(\operatorname{Holds}\left(A\left(y, o_{y}, \Gamma_{y}, \Pi_{y}\right), z\right) \wedge P \text { Known }\left(\Pi_{y}, \pi_{P}, \pi_{R}\right)\right)\right)\right] \wedge \\
& \left.\quad P F u s i o n\left(o, \Pi_{P}, \Gamma^{\prime}, s\right) \wedge\left(\exists \pi_{P}\right)\left(\pi_{P} \in \Pi_{P} \wedge x=\pi_{P}\right)\right)
\end{aligned}
$$

Using the same parameters as the other two symbol anchoring functionalities, the variables $x, f$ and $f^{\prime}$ of macro $\Delta_{N}$ are existentially quantified in the Axiom (5.23). This way, the purpose of macro $\Delta_{N}$ is to determine a new object symbol $x$, the fluents $f$ and $f^{\prime}$ to be removed from and added to the hypothesis representation, respectively, for the given block of percepts $\Pi_{P}$, situation $s$ and hypothesis $z$. As this functionality introduces a new object symbol, anchor and correspondence for a new percept, nothing has to be retracted from the previous hypothesis representation $z$, such that $f=\emptyset$. The new anchor $f^{\prime}$, which will be introduced to the successor hypothesis $z^{\prime}$, is initialized with the object category $o$ and block of percepts $\Pi_{P}$ under consideration. The predicate PFusion yields the new best perceptual estimate $\Gamma^{\prime}$ for the new anchor $f^{\prime}$ by fusing the perceptual signatures of all percepts of the block $\Pi_{P}$ into a single aggregate signature.

The conjunct in squared brackets in the middle of Formula (5.30) poses the condition that a percept of the given block of percepts must not be associated with some anchor already, because this would allow the functionality Track to apply, too. The condition $x \geq \pi_{P}$ constrains the new object identifier - being a natural number - with respect to the percept identifiers. Together with the last conjunct $\left(\exists \pi_{P}\right)\left(\pi_{P} \in \Pi_{P} \wedge x=\pi_{P}\right)$, both conditions determine $x$ to equal the maximal percept identifier of block $\Pi_{P}$. Apparently, new object identifiers can only be of sort PERCID, which is reserved solely for new objects that are experienced during runtime. Thus, no conflict with the object symbols of sort $\mathcal{X}$ may occur thanks to Formula (5.8).

The method of determining the new object identifier $x$ implemented in macro $\Delta_{N}$ guarantees that each new block of percepts is assigned a unique object identifier. Recall from Section 5.4.2 that the predicate PBlock only holds for a block of associated percepts, while the union of these blocks altogether forms a partition of the full set of available percepts $\widehat{\Pi}_{s}$ in situation $s$. Our approach achieves the desired uniqueness of the new object identifier as follows. First, identifiers of percepts are unique (cf. Section 5.3.1). Second, different blocks of the partition $\widehat{\Pi}_{s}$ comprise disjunct sets of percept identifiers (cf. Section 5.4.2). Therefore, any correspondence between a block of percepts and an object symbol is unique, if one of the percept identifiers of this block of percepts is used as the object symbol. Since the macro $\Delta_{N}$ chooses the maximal value of the percept identifiers of each block of percepts, the choice is deterministic. In other words, from the fact that any two different blocks $\Pi_{1}$ and $\Pi_{2}$ of percepts are disjunct sets of percept identifiers, it follows that $\max \Pi_{1} \neq \max \Pi_{2}$ (cf. Formula (5.21)). Hence, the macro $\Delta_{N}$ determines unique 
object symbols, which is a prerequisite for consistent correspondences. The consistency of symbol anchoring hypotheses will be discussed below.

The macro $\Delta_{N}$ employs the predicate PFusion in order to fuse all available perceptual information of the percepts of the given block $\Pi_{P}$ into a single aggregate structure $\Gamma^{\prime}$, which becomes the initial, best perceptual estimate of the anchor of the new object. The definition of the predicate PFusion was derived from the predicate AnchorUpdate as a specific instance where the previous anchor is set to nil. In contrast to predicate AnchorUpdate, Formula (5.31) may neglect to distinguish between static and dynamic perceptual attributes, since PFusion does not need to update a previous record of perceptual information. Under these conditions, the definition of predicate AnchorUpdate reduces to the following Formula (5.31) implementing PFusion.

$$
\begin{gathered}
\text { PFusion : OBJCAT } \times \mathcal{P}(\text { PERCID }) \times \text { PERCSIG } \times \text { SIT } \\
\text { PFusion }\left(o, \Pi_{P}, \Gamma^{\prime}, s\right) \equiv \text { AnchorUpdate }\left(o, \emptyset, \emptyset, \Pi_{P}, \Gamma^{\prime}, \Pi_{P}, s\right) \equiv \\
\left(\left(a,\left(b^{\prime}, t^{\prime}\right)\right) \in \Gamma^{\prime} \equiv\right. \\
{\left[\pi_{P} \in \Pi_{P} \wedge P\left(\pi_{P}, o, \Gamma_{P}, \pi_{R}, s\right) \wedge \gamma_{P} \in \Gamma_{P} \wedge \gamma_{P}=\left(a,\left(b_{P}, t_{P}\right)\right)\right.} \\
\left.\supset b_{P} \leq b^{\prime} \wedge t_{P} \geq t^{\prime}\right] \wedge \\
\left(\exists \gamma, b, t, \pi_{P}, \Gamma_{P}, \pi_{R}\right)\left[\gamma=(a,(b, t)) \wedge b^{\prime}=b \wedge P\left(\pi_{P}, o, \Gamma_{P}, \pi_{R}, s\right) \wedge\right. \\
\left.\pi_{P} \in \Pi_{P} \wedge \gamma \in \Gamma_{P}\right] \wedge \\
\left(\exists \gamma, b, t, \pi_{P}, \Gamma_{P}, \pi_{R}\right)\left[\gamma=(a,(b, t)) \wedge t^{\prime}=t \wedge P\left(\pi_{P}, o, \Gamma_{P}, \pi_{R}, s\right) \wedge\right. \\
\left.\left.\pi_{P} \in \Pi_{P} \wedge \gamma \in \Gamma_{P}\right]\right) \wedge \\
\left(\left(a,\left(b^{\prime}, t^{\prime}\right)\right) \in \Gamma^{\prime} \supset b^{\prime}<t^{\prime}\right)
\end{gathered}
$$

The definition of predicate PFusion requires for any perceptual attribute $a$ that a record $\left(a,\left(b^{\prime}, t^{\prime}\right)\right)$ is only part of the new perceptual estimate $\Gamma^{\prime}$ if the following conditions hold:

1. Each percept providing information on the perceptual attribute $a$ constrains the new perceptual estimate of this attribute to be at least as precise as the perceptual signature $\gamma_{P} \in \Gamma_{P}$ of the percept.

2. Since the interval representation of the new perceptual estimate must not be arbitrarily precise, but has to be sustained by some percept, the two conjuncts in the middle of Formula (5.31) require that the new limits must equal some of the given limits. In other words, predicate PFusion must not infer more precise information than the percepts report.

3. The last conjunct makes sure that the new representation is consistent with the assumption defined in Formula (5.4) (cf. Section 5.4.5).

Comparing the uni-modal and the multi-modal versions of the symbol anchoring functionality New, both of them implement two main tasks likewise. In both versions, the functionality New specifies its applicability conditions. Furthermore, this functionality introduces a new, unique anchor representation and object symbol for the given percepts. On top of that the multi-modal functionality New fuses all associated percepts forming the new perceptual estimate.

This section concludes the definition and explanation of our multi-modal symbol anchoring axiom of Formula (5.23) and of all its components. We combine all the above-mentioned axioms and constraints of our multi-modal approach in the set $\Sigma_{m m s a}$ of axioms called the multi-modal symbol anchoring axioms.

$$
\begin{aligned}
\Sigma_{m m s a} \stackrel{\text { def }}{=} & \{(5.2),(5.3),(5.4),(5.5),(5.7),(5.8)\} \\
& \cup\{(5.10),(5.11),(5.13),(5.22),(5.23)\}
\end{aligned}
$$




\subsubsection{Complexity of Hypothesis Space}

The complexity of the successor hypothesis space after applying the multi-modal symbol anchoring Axiom (5.23) depends very much on the environmental situation, the application domain, the robot's capacity of recognition etc. However, let's discuss the effects of our symbol anchoring approach on the complexity of the representation given some percepts in general. First of all, due to the assumptions we defined in Section 5.3.1 and due to the definition of the symbol anchoring Axiom (5.23) all percepts of the same block of percepts determined by predicate PBlock correspond with the same object symbol. The Axiom (5.23) complies with this concept as it quantifies the object symbol $x$ existentially outside of the macros $\Delta_{T}, \Delta_{F}$ and $\Delta_{N}$. Hence, for the purpose of discussing the complexity of the successor hypothesis space, it suffices to consider correspondences of blocks of associated percepts, instead of individual percepts.

As we mentioned in the above Section 5.4.4, if the functionality Track is applicable to a certain block of percepts, then Axiom (5.23) defines this as the only applicable functionality. In accordance with the definition of macro $\Delta_{T}$ in Formula (5.25), only a single object symbol may correspond with the given block of percepts in the successor hypotheses if functionality Track applies, i. e., the functionality Track causes no change to the complexity of the hypothesis space. However, while percepts are valid and unique for all hypotheses of the given situation, individual hypotheses include independent representations of correspondences with object symbols and knowledge about them in general. Therefore, in case the functionality Track is applicable to a certain block of percepts for a certain hypothesis, Track may not be applicable to the same block of percepts for a different hypothesis of the same situation. Hence, the discussion on the complexity of the successor hypothesis space is to be seen from the perspective of an individual hypothesis only. The same applies to the discussion on complexity of the uni-modal symbol anchoring axiom in Section 4.2.5.

Only if the functionality Track does not apply to a certain block of percepts and a certain hypothesis, the functionality Find may do so in this situation. The functionality Find presupposes the existence of some anchor, object symbol and symbolic knowledge that all match the perceptual information of the given block of percepts. Accordingly, the number of objects, which the functionality Find could associate with the given block of percepts, strongly depends on the characteristics and the number of already known objects represented in the given hypothesis, the specificity of the information of the percepts and the specificity of the perceptual information in the object's anchor as well as of the symbolic knowledge on that object. The more similar the objects of the environment appear to the cognitive robot, and the less specific the symbolic and perceptual information on the objects is, and the less precise the percepts of the physical objects are, the more matching candidate objects does the functionality Find determine. In particular, functionality Find may also determine that no correspondence could be found for a certain block of percepts for the hypothesis at hand. If Find determines more than one matching object symbol for the given block of percepts, the multi-modal symbol anchoring axiom introduces an additional successor hypothesis for each possible, consistent correspondence.

In contrast to Track, the applicability of functionality New is restricted to those blocks of percepts that are not reported to be associated with some anchor. However, the symbol anchoring Axiom (5.23) introduces just one unique, new correspondence if functionality New applies. In other words, depending on whether functionality New is applicable, it introduces no or one successor hypothesis of correspondences for the given block of percepts and the original hypothesis.

In summary, the complexity of the successor hypothesis space generated by our multi-modal symbol anchoring approach is the same or higher than the respective complexity of our uni-modal approach, depending on the object recognition system reporting which percepts are associated. On the other hand, our multi-modal approach may prevent a number of possible successor hypotheses from entering the hypothesis space in practice due to obtaining richer perceptual information from additional sensors observing the objects of the robot's environment. 


\subsubsection{Eliminating Inconsistent Hypotheses}

At first glance it may appear as if the complexity of the hypothesis space is ever growing with each symbol anchoring transaction. Conversely, the multi-modal symbol anchoring Axiom (5.23) only specifies those hypotheses explicitly that get updated, that are carried over to the next situation or which are generated. Apart from those possible transitions, no further hypothesis persists in the successor situation. In particular, all those hypotheses of correspondences which do not allow a transition to a consistent successor hypothesis become neglected completely. Basically the same concept of eliminating inconsistent hypotheses applies to multi-modal symbol anchoring as we described it for the uni-modal case in Section 4.2.6.

The following example demonstrates the rationale behind our approach eliminating inconsistent hypotheses of correspondences. Assume that a certain correspondence between an object symbol and a block of percepts was established previously by mistake due to a vague symbolic description or an imprecise perceptual estimate in the anchor or due to percepts of insufficient detail. Then, consider that the recognition system reports that the same object was successfully tracked and provides further perceptual information on it. Our symbol anchoring functionality Track applies in this case, but fails due to contradicting new perceptual data that disagree with the symbolic description of the object or the perceptual estimate accumulated so far. As a result, the symbol anchoring Axiom (5.23) cannot update this hypothesis of correspondence towards the successor situation, such that it becomes eliminated. Taking all available information into account in order to disambiguate the situation is a fundamental cognitive concept, which Harnad [2005] circumscribed as: "Information, as we learn from formal information theory, is something that reduces the uncertainty among alternatives."

Also recall from Section 4.2.6 that our approach guarantees the persistence of some alternative hypothesis if a hypothesis has to be eliminated due to an inconsistency. In particular, the actually correct one is supposed to be among the successor hypotheses. The result of the uni-modal version applies likewise to our multi-modal approach, because both are based on the same foundational concepts described in Chapter 3. In summary, our multi-modal symbol anchoring Axiom (5.23) determines all potential hypotheses of correspondences, and identifies and retracts inconsistent hypotheses automatically.

\subsection{Preferences among Alternative Hypotheses}

A hypothesis should be considered a particular interpretation of the situation the world and the cognitive robot are in, especially regarding correspondences between the robot's internal object symbols and the physical objects. Both our uni-modal and multi-modal approaches maintain multiple hypotheses of correspondences in general for the purpose of resolving ambiguity of perception and symbol anchoring (cf. Section 3.10). However, when it comes to planning and execution of actions in the real world, the cognitive robot has to decide for the one hypothesis or the other eventually. We proposed to base the decision on certain preference criteria, which we defined in abstract terms in Section 4.3 for our uni-modal symbol anchoring approach. We have also introduced a so-called belief state designating the most preferred hypothesis of correspondences.

All the definitions of Section 4.3 are based on our uni-modal approach. Nonetheless, these concepts apply likewise to our multi-modal approach of Chapter 5, but require a few, slight adaptations. Since these adaptations are straightforward and do not showcase significant advances, we skip to explicate their respective multi-modal version at this point.

\subsection{Characteristics of Our Multi-Modal Approach}

We dedicate this section to demonstrating that our multi-modal symbol anchoring approach complies with crucial characteristics of symbol anchoring in general. We will also show here the important result of the uni-modal approach being a special case of the multi-modal approach 
(cf. Section 5.6.4). This result allows to infer that those characteristics, proven to hold for our multi-modal approach in the subsequent sections, do likewise hold for the uni-modal axioms.

\subsubsection{Consistency of Hypotheses}

Our approach requires that all hypotheses of all situations are consistent with respect to first, all available information attributed to the considered hypothesis in general and second, with respect to the criteria defined by the predicate Consistent in particular. Regarding the first requirement, our approach eliminates inconsistent hypotheses of correspondences automatically as explained in Section 5.4.8. In this section we are going to show that all hypotheses computed by our approach actually comply with the second requirement.

Lemma 5.2 All hypotheses of correspondences computed by the multi-modal symbol anchoring axiom of Formula (5.23) are consistent with respect to the constraints of predicate Consistent, i.e. ${ }^{1}$

$$
(\forall)(\operatorname{KState}(s, z) \supset \text { Consistent }(z))
$$

Proof The idea of the proof is the following. Given that the initial hypotheses are consistent, and given that any symbol anchoring transaction of any hypothesis yields consistent successor hypotheses only, then it follows that all hypotheses satisfy the consistency criteria. We disregard the Formula (5.13) for a moment, which implements the requirement that all hypotheses associated with the initial situation have to be consistent.

Induction base: All initial hypotheses are required to comply with the Formulas (5.10) and (5.11). Accordingly, initial hypotheses are consistent due to the following reasons.

1. The Signature (5.9) of anchor fluents imposes that object symbols only accept values of the sort $\mathcal{X} \cup$ PERCID.

2. The Formula (5.10) disallows any fluents with object symbols of the sort PERCID to exist in hypotheses of the initial situation. Since the Formula (5.11) has to hold for all anchor fluents of initial hypotheses, these anchor fluents may only take object symbols of the sort $\mathcal{X}$.

3. The Formula (5.11) requires $\Gamma=\emptyset$ and $\Pi=\emptyset$ to hold for any anchor fluent of the initial hypothesis.

4. Moreover, the Formula (5.11) entails the existence of exactly one instance of an anchor fluent for each individual object symbol $x: \mathcal{X}$ in each initial hypothesis.

Now, why do all initial hypotheses $z$ satisfy the predicate Consistent $(z)$ according to its definition in Formula (5.12)? The first conjunct of predicate Consistent requiring uniqueness of anchor fluents with respect to object symbols holds true thanks to the above item 4 . The second conjunct of predicate Consistent considers anchor fluents which bear a non-empty last component - the set $\Pi$ of associated percept identifiers - and requires uniqueness of anchor fluents with respect to $\Pi$ of initial hypotheses. Due to Formula (5.11) constraining $\Pi=\emptyset$ to hold for all anchor fluents as we pointed out in the above item 3 , the second conjunct of the predicate Consistent holds true as well. Hence,

$$
\{(5.10)\} \cup\{(5.11)\} \models(\forall z)\left(\operatorname{KState}\left(S_{0}, z\right) \supset \text { Consistent }(z)\right)
$$

Induction hypothesis: Suppose a situation $s$ where all associated states $z$ are consistent with respect to the predicate Consistent.

Induction step: Next, this part of the proof shows why the consistency of hypotheses is preserved when the multi-modal symbol anchoring Axiom (5.23) is applied to consistent, original

\footnotetext{
${ }^{1}$ Obviously, the symbol anchoring representation may remain consistent only if no symbolic reasoning system of the cognitive robot manipulates it in an inconsistent way.
} 
hypotheses only. We start by briefly summarizing the features of the top-level Axiom (5.23), which implements the consistency constraints on successor hypotheses. For each existing hypothesis of the given situation $s$ the symbol anchoring axiom determines those correspondences that need to be and actually can be updated using some functionality, while all other correspondences remain untouched. The former kind of correspondences are stored temporarily in an auxiliary state term for the sole purpose of verifying the consistency of the whole set of new correspondences. For each of those correspondences determined to be updated, the appropriate symbol anchoring functionality determines the new correspondence.

The constraints on the state term $\vartheta$ in the Axiom (5.23) ensure that first, each correspondence to be updated gets replaced with exactly one new correspondence in the successor hypothesis representation $z^{\prime}$. Second, object symbols used in the updated correspondences of state $\vartheta$ are required to be unique. And third, two different blocks of percepts must not correspond with the same object symbol among the correspondences of state $\vartheta$. This way, the constraints of the Axiom (5.23) disallow that multiple symbol anchoring functionalities are applied to the same block of percepts or to the same object symbol within a successor hypothesis. The above conditions can directly be seen to constrain the legitimate correspondences of a successor hypothesis. Altogether, these conditions satisfy the consistency criteria defined by the predicate Consistent for the correspondences of state $\vartheta$. While these consistency conditions only concern the correspondences of state $\vartheta$, all the correspondences of the complete successor hypothesis are required to comply with the consistency criteria. In order to show this, we need to have a closer look at each of the three functionalities.

In case the symbol anchoring functionality Track is applicable to a given correspondence of a certain hypothesis and situation, the macro $\Delta_{T}$ determines the anchor fluent to be retracted and the new anchor fluent replacing the previous one uniquely. As can be seen in the Formula (5.25) defining Track, the same object symbol is reused, while the set of associated percept identifiers is amended. Reusing the same object symbol for a correspondence when updating a consistent hypothesis leads to a consistent successor hypothesis, if this symbol is not employed by any other functionality for some other correspondence. Both the functionality Find and New include the condition

$$
\neg(\exists)\left(\operatorname{Holds}\left(A\left(y, o_{y}, \Gamma_{y}, \Pi_{y}\right), z\right) \wedge P \operatorname{Known}\left(\Pi_{y}, \pi_{P}, \pi_{R}\right)\right.
$$

for a new percept $\pi_{P}$ in order to prevent another correspondence to associate the object $y$ which is being tracked already. Likewise, this condition ensures that a block $\Pi_{y}$ of associated percepts being tracked does not get employed in the functionality Find or New. Hence, the functionality Track preserves the consistency criteria for the whole successor hypothesis if applied to a consistent hypothesis.

The functionality Find restricts its applicability internally in Formula (5.29) to such object symbols that are not already associated with any percepts on the one hand, and to such blocks of percepts that are not associated already with any object symbol on the other hand. Hence, the functionalities Find and Track cannot interfere, because their applicability conditions are mutually exclusive for each correspondence, such that no additional correspondence for some already anchored object symbol or percept may be introduced which would give rise to an inconsistency. The above-mentioned consistency constraints on the state term $\vartheta$ in the symbol anchoring Axiom (5.23) prevent that the functionality Find may be applied multiple times to the same object symbol or to the same block of percepts within the same successor hypothesis. Since the internal applicability conditions of functionality Find in Formula (5.29) regard the whole hypothesis under consideration - not only the correspondences of state $\vartheta$ waiting to be updated - the consistency of a given hypothesis is preserved by functionality Find for the successor hypothesis.

Similar to Find, the functionality New includes the mutual-exclusive applicability condition with regard to functionality Track, too. Furthermore, the constraints on the updated correspondences in the symbol anchoring Axiom (5.23) prevent that the functionalities New and Find could yield multiple, different correspondences with the same block of percepts. Due to the fact that functionality New assigns object symbols solely of sort $\mathcal{X}$, which no other functionality employs, and since New guarantees that each employed symbol is not already in use for some previous cor- 
respondence of the considered hypothesis, the functionality New introduces correspondences with unique object symbols only. Altogether, the above showed, that given a consistent hypothesis, the symbol anchoring functionality New preserves the consistency for the successor hypothesis.

If no percept is available, naturally, the application of the multi-modal symbol anchoring Axiom (5.23) preserves the consistency of all hypotheses. This can be seen easily, since the update of the hypothesis representation becomes simplified from $z^{\prime}=z-\vartheta^{-}+\vartheta^{+}$to $z^{\prime}=z$ in that particular case, i. e., the hypothesis space remains untouched.

Concluding what we have got so far, it follows that the Axiom (5.23) of our multi-modal symbol anchoring approach preserves the consistency of hypotheses altogether.

\subsubsection{Groundedness of Correspondences}

We discussed the concept of groundedness of correspondences between object symbols and the physical objects they denote in Section 4.2.8 above. Groundedness in terms of symbol anchoring denotes a measure of the following two aspects.

1. Is the considered anchor grounded in the considered situation? This is the question whether a suitable percept has established a correspondence between the symbol and the physical object in the considered situation.

2. If this is the case, how well does the symbolic information about the object match the corresponding real-world counterpart? This question is strongly correlated to the more tempting question, whether a given correspondence is actually correct. ${ }^{2}$

Apparently, groundedness cannot resolve the question whether the correspondence between an object symbol and the given percept is actually correct. We consider groundedness a property of an object saying whether the symbol anchoring system provides a grounded anchor for the given object symbol. The reader may want to consult the Section 4.2.8 for further background information.

First and foremost, the property groundedness of a correspondence serves the purpose of a precondition of an action on the considered object of the environment. Due to the differences to the representation of our uni-modal approach, we redefine the predicate IsGrounded for use in our multi-modal approach as follows.

$$
\text { Is Grounded }: \mathcal{X} \cup \text { PERCID } \times \text { SIT } \times \text { STATE }
$$

$$
\begin{aligned}
& \text { IsGrounded }(x, s, z) \equiv \\
& \begin{array}{l}
(\exists)\left(\operatorname{KState}(s, z) \wedge \operatorname{Holds}\left(A\left(x, o, \Gamma_{A}, \Pi_{A}\right), z\right) \wedge P\left(\pi_{P}, o, \Gamma_{P}, \pi_{R}, s\right) \wedge\right. \\
\left.\quad \text { KKnown }\left(\Pi_{A}, \pi_{P}, \pi_{R}\right)\right)
\end{array}
\end{aligned}
$$

This predicate determines whether the correspondence between a given object symbol and an object of the environment is grounded on some suitable percept in the given situation and hypothesis. In comparison with the definition of the uni-modal predicate IsGrounded in Formula (4.36), the multi-modal version makes use of the benefits of multi-modal perception in general: Multiple percepts associated with the given object symbol may provide the grounded correspondence. Moreover, these percepts may have been measured by means of an arbitrary sensing device. A wide diversity of sensor equipment typically increases the availability of some measurement of the object of interest due to individual measurement ranges, precision and tracking capabilities in practice.

\footnotetext{
${ }^{2}$ See Section 2.2 for the reason why it is also not recommended to put a human, external observer in the loop in order to examine the situation and to assess whether or not a certain correspondence is correct. Our approach does not ignorantly hit this pitfall of an inappropriate strategy to approach the philosophical problem of symbol grounding.
} 
Note that the Formula (5.33) is based on the assumptions about the reference percept we made in Section 5.3.1, in particular the assumption about the object recognition system to report which previous percept a current percept refers to, in case the object could be tracked successfully and reliably. The predicate PKnown simply determines whether such a relation with some previous percept exists (cf. Formula (5.26)). In summary, the predicate IsGrounded $(x, s, z)$ tells us whether the anchor of the given object symbol $x$ is grounded on some current percept, or in practical terms, whether action execution on the given object symbol may commence in situation $s$.

\subsubsection{Each Percept Is Assigned Some Symbol}

The Section 3.4 conveyed our fundamental concept that a symbol anchoring approach should ensure to associate each percept with some object symbol. Representing the correspondence between the percept and the object symbol in form of an anchor fluent, symbol anchoring then is a matter of determining the right object symbol. ${ }^{3}$ Thus, no percept gets neglected. Since each percept may stand for the very object of interest and thus represents a potential candidate, this concept contributes to completeness of the set of successor hypotheses.

Lemma 5.3 Our multi-modal symbol anchoring approach makes sure to assign some object symbol to every available percept. More precisely, given a situation $s$ where some percept $\pi$ is available, i. e., $(\exists) P\left(\pi, o, \Gamma, \pi_{R}, s\right)$ holds, each successor hypothesis $z^{\prime}$ resulting from the application of the multi-modal symbol anchoring Axiom (5.23) in situation s with KState (Do $($ AnchorM $\left.(o), s), z^{\prime}\right)$ will include an anchor fluent $A\left(x, o, \Gamma_{A}, \Pi_{A}\right)$ that associates some object symbol $x$ with the percept $\pi$ with $\pi \in \Pi_{A}$.

Proof Recall that the set $\widehat{\Pi}_{s}$ comprises all percepts available in situation $s$, i.e., $\widehat{\Pi}_{s}$ represents the set of percept identifiers which the percept predicate $P / 5$ holds for in situation $s$ (cf. Formula (5.14)). Thus, any percept of a certain situation $s$ is included in the set $\widehat{\Pi}_{s}$. Our multi-modal symbol anchoring Axiom (5.23) considers the set $\widehat{\Pi}_{s}$ as a partition, where the predicate PBlock serves to identify disjoint blocks of associated percepts uniquely (cf. Section 5.4.1). According to the proof in Section 5.4.2 the predicate PBlock determines a mathematical partition of the set $\widehat{\Pi}_{s}$ correctly. Hence, the union of all blocks of percepts amounts to the complete set $\widehat{\Pi}_{s}$. Therefore, each percept of the considered situation $s$ is part of exactly one block $\Pi_{P}$ of percepts with $\operatorname{PBlock}\left(\Pi_{P}, s\right)$. The implication $\operatorname{PBlock}\left(\Pi_{P}, s\right) \supset \ldots$ within the symbol anchoring Axiom (5.23) ensures that every block of percepts is taken into account for symbol anchoring, and as such is every percept, too. The Axiom (5.23) applies the disjunction $\Delta_{T}(\ldots) \vee \Delta_{F}(\ldots) \vee \Delta_{N}(\ldots)$ of the three functionalities Track, Find and New, respectively, to each block of percepts, and at least one functionality is required to hold true and to comply with the rest of the considered hypothesis in order to update the hypothesis consistently. As we described in Section 5.4.3 either Track or the other two functionalities, New and Find, may be applicable to a certain block of percepts. Each functionality verifies some additional consistency conditions. The functionality Track reuses the same object symbol for the correspondence with the considered block of percepts in the successor hypothesis. The functionality New assigns a fresh, unique object symbol to the considered block of associated percepts introducing the new correspondence into the successor hypothesis. The functionality Find associates the given block of percepts with some appropriate object symbol if applicable. Regardless of whether the block of percepts is being tracked or not, the disjunction of the three functionalities determines an appropriate object symbol to associate with the percepts if the update of the correspondence is consistent. Since we are only interested in consistent successor hypotheses, we can neglect the inconsistent ones in this analysis.

In case the functionalities Track and Find were applied, a consistent successor hypothesis is obtained from an original hypothesis by replacing an anchor's outdated counterpart. Obviously, no correspondence gets lost in this process and the percept becomes firmly connected with some object

\footnotetext{
${ }^{3}$ Determining the right object symbol of a correspondence with a percept obviously constitutes the main symbol anchoring task, which our approach solves very successfully.
} 
symbol. The functionality New simply adds an anchor representing the new correspondence to the outdated hypothesis in order to gain the successor hypothesis. This way, a percept is guaranteed to become associated with some object symbol, too.

Altogether this shows that each individual percept is taken into account when applying our multi-modal symbol anchoring Axiom (5.23) in any situation, as each percept is associated with some (appropriate) object symbol in each consistent successor hypothesis.

\subsubsection{Relation between Uni- and Multi-Modal Approaches}

We have presented our uni-modal approach to the symbol anchoring problem in Chapter 4 above and our multi-modal approach in this Chapter 5. Both versions share a common set of objectives, foundational concepts and assumptions (cf. Chapter 3). However, their respective implementations differ significantly, yet they both have a similar semantics. In this section we are going to show that the multi-modal approach generalizes the uni-modal one. The multi-modal version adds multimodal perception on top of the expressiveness and functionality of the uni-modal version. Multimodal perception enables the cognitive robot to deal with multiple sensors that yield multiple, individual percepts per object of the world. In terms of the logical relation between both versions, this section will show that the uni-modal approach is a special case of the multi-modal approach as expected.

Theorem 5.4 The uni-modal symbol anchoring approach is a special case of the multi-modal approach. Hence, the uni-modal symbol anchoring Axioms $\Sigma_{u m s a}$ entail the multi-modal symbol anchoring Axiom (5.23) such that the following relation holds:

$$
\Sigma_{\text {umsa }} \models(5.23)
$$

Proof The proof of this theorem regarding the Axiom (4.15) comprises several steps. The Figure 5.3 displays an informal overview of the structure of this part of the proof. ${ }^{4}$ Besides the Axiom (4.15), the logical relation of all other axioms of $\Sigma_{u m s a}$ with their corresponding multimodal counterpart is rather straightforward and will be explained below.

Before we can derive the logical relation between our uni-modal and the multi-modal approach, their respective representations need to be aligned as they differ slightly. For this purpose, we will introduce the transformation function $T$ that translates the somewhat limited uni-modal language into the multi-modal language in a semantically equivalent way. The following, simple rewriting rules implement this function $T$.

- For quantors $Q$, variables $\vec{x}$, and formulas $F$ we define the transformation function $T$ :

$$
T((Q \vec{x}) F) \stackrel{\text { def }}{=}(Q \vec{x}) T(F)
$$

Obviously, this rule of the transformation function does not change the structure or the meaning of the given formula.

- The result of applying function $T$ to the formulas $F$ and $G$ being connected with a binary logical operator - including the connection function $\circ$ of the Fluent Calculus is defined as:

$$
T(F \cdot G) \stackrel{\text { def }}{=} T(F) \cdot T(G)
$$

Accordingly, the function $T$ does not modify the structure or the meaning of $F \cdot G$.

- For negated formulas $F$ :

$$
T(\neg F) \stackrel{\text { def }}{=} \neg T(F)
$$

Again, neither the structure nor the meaning is altered when applying this rule.

\footnotetext{
${ }^{4}$ We abbreviate uni-modal with um. and multi-modal with $\mathrm{mm}$.
} 


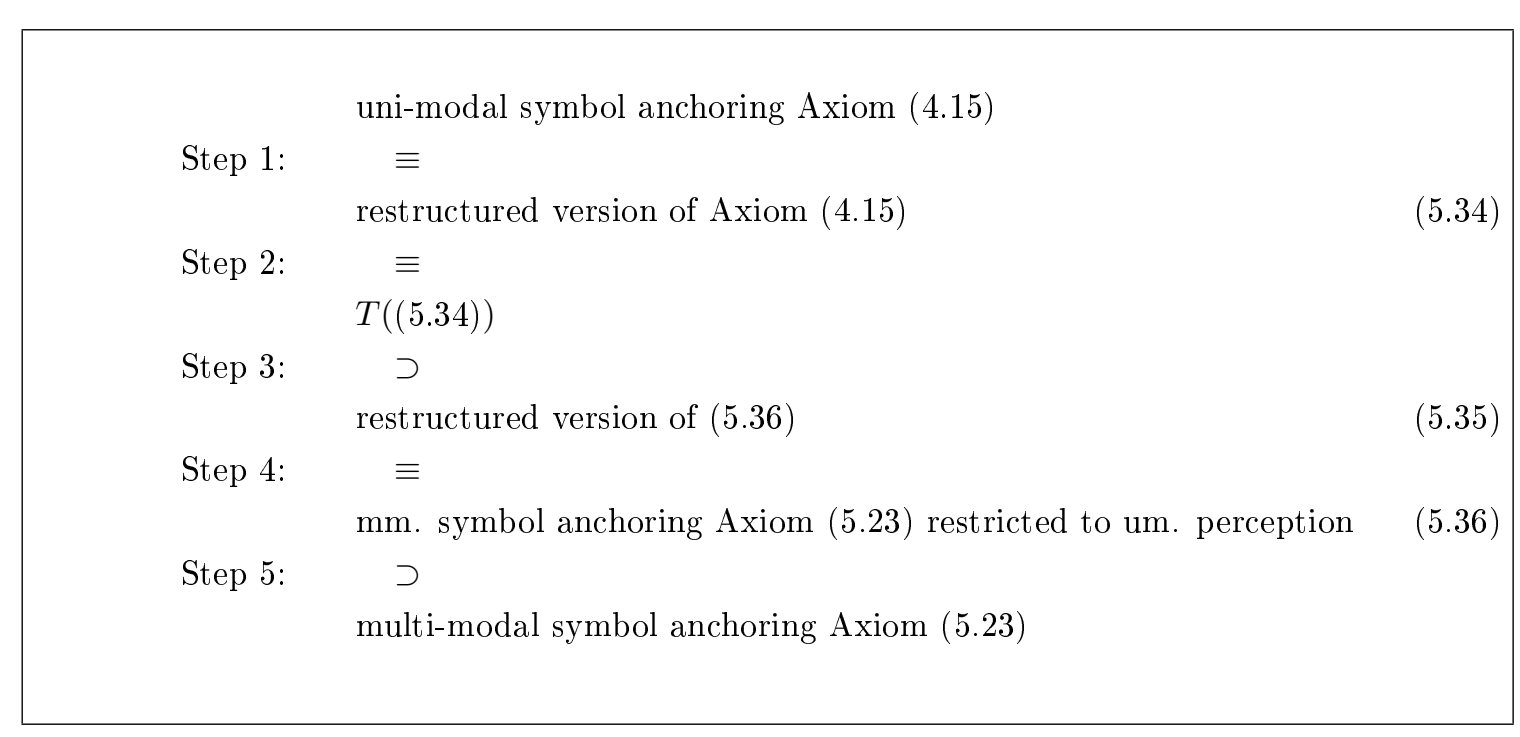

Figure 5.3: Structure of the proof of the logical relation between the uni-modal and the multimodal symbol anchoring axioms (informal).

- Now, the interesting part of the definition of function $T$ begins. Recall the Signature (4.8) of the uni-modal anchor fluent $A$

$$
A: \mathcal{X} \cup \text { PERCID } \times \text { OBJCAT } \times \text { PERCSIG } \times \text { PERCID } \cup\{\perp\} \mapsto \text { FLUENT }
$$

and the Signature (5.9) of the multi-modal version of fluent $A$ :

$$
A: \mathcal{X} \cup \text { PERCID } \times \text { OBJCAT } \times \text { PERCSIG } \times \mathcal{P}(\text { PERCID }) \mapsto \text { FLUENT }
$$

They differ only in the sort of the last component. While the uni-modal anchor fluent represents a single percept identifier or the constant $\perp$ in the last component, the multi-modal anchor fluent represents a set of percept identifiers therein. However, both the constant $\perp$ and the empty set $\emptyset$ mean likewise that the anchor is not grounded on some percept. Therefore, uni-modal anchor fluents may be rewritten to the multi-modal, semantically equivalent form using in the following rule regarding fluents $f$ :

$$
T(f) \stackrel{\text { def }}{=} \begin{cases}A(x, o, \Gamma, \emptyset) & \text { if } f=A(x, o, \Gamma, \perp) \\ A(x, o, \Gamma,\{\pi\}) & \text { else if } f=A(x, o, \Gamma, \pi) \\ f & \text { otherwise }\end{cases}
$$

Other fluents than anchor fluents are not modified by function $T$.

- Comparing the definition of the uni-modal percept predicate $P / 4$ of Signature (4.1)

$$
P: \text { PERCID } \times \text { OBJCAT } \times \text { PERCSIG } \times \text { SIT }
$$

with the one of the multi-modal percept predicate $P / 5$ of Signature (5.1)

$$
P: \text { PERCID } \times \text { OBJCAT } \times \text { PERCSIG } \times \text { PERCID } \cup\{\perp\} \times \text { SIT }
$$

they only differ in the additional, fourth component of the multi-modal predicate $P / 5$. In contrast to the multi-modal predicate $P / 5$, the uni-modal approach does not use reference percepts. The multi-modal approach uses the constant $\perp$ for the purpose of representing that no reference percept is available. Thus, the following definition of function $T$ translates 
the uni-modal percept predicate to the language of the multi-modal approach in a semantically equivalent way. Function $T$ is defined for predicates $p(\vec{x})$ with variables $\vec{x}$ as follows, leaving other predicates than $P$ untouched.

$$
T(p(\vec{x})) \stackrel{\text { def }}{=} \begin{cases}P(\pi, o, \Gamma, \perp, s) & \text { if } p(\vec{x})=P(\pi, o, \Gamma, s) \\ p(\vec{x}) & \text { otherwise }\end{cases}
$$

- The uni-modal and the multi-modal approaches presented in this work employ the two epistemic actions AnchorU and AnchorM, respectively, for computing the successive symbol anchoring hypothesis space. In order to allow a logical comparison of their respective knowledge update axioms, we need to equalize their syntactic names. Changing the name of the uni-modal symbol anchoring action does not alter the semantics of computing the successor hypothesis space.

$$
T(\operatorname{Anchor} U(\vec{x})) \stackrel{\text { def }}{=} \operatorname{AnchorM}(\vec{x})
$$

Thus, the translation function $T$ simply renames the uni-modal symbol anchoring action preserving semantics.

We explained along each one of the above rules of the function $T$ why it preserves the semantics. We are particularly interested in using the transformation function $T$ for translating our uni-modal symbol anchoring axioms $\Sigma_{u m s a}$ of Formula (4.32) into the language of the multi-modal approach. This yields the first auxiliary result of this proof: Translating a well-formed formula $F_{u} \in \mathcal{L}_{u}$ of the uni-modal language $\mathcal{L}_{u}$ using the function $T$ yields a corresponding, semantically equivalent formula in the multi-modal language $\mathcal{L}_{m}$, i. e.

$$
\models\left(T\left(F_{u}\right) \equiv F_{u}\right)
$$

Next, we will examine each axiom $F \in \Sigma_{u m s a}$ of the uni-modal symbol anchoring axioms $\Sigma_{u m s a}$ comparing $F$ - or its translation $T(F)$ — with the corresponding sibling of the multi-modal symbol anchoring axioms $\Sigma_{m m s a}$. We recognize the following particular relations.

- The uni-modal Formula (4.9) restricting the sorts $\mathcal{X}$ and PERCID to be disjoint equals the corresponding, multi-modal Formula (5.8), i. e., $\models(4.9) \equiv(5.8)$.

- The uni-modal Formula (4.13) is equal to the multi-modal Formula (5.13), both restricting the initial hypotheses of correspondences likewise. Hence, $\models(4.13) \equiv(5.13)$.

- The translated uni-modal Formula (4.3) represents a particular instance of the multi-modal Formula (5.3) with $\pi_{R}=\perp$, such that $\mid=T((4.3)) \supset$ (5.3) holds.

- The uni-modal constraint on perceptual signatures of Formula (4.4) is a specific instance of the corresponding multi-modal one with $\pi_{R}=\perp$, i. e. $\models T((4.4)) \supset(5.4)$.

- Similarly, the uni-modal constraint of Formula (4.5) on perceptual attributes representing uni-modal distributions is obviously a particular instance of the corresponding Formula (5.5) with $\pi_{R}=\perp$ such that $\models T((4.5)) \supset(5.5)$.

- Likewise, the uni-modal Formula (4.6) is a particular instance of the multi-modal Formula (5.2) with $\pi_{R}=\perp$, i.e. $\models T((4.6)) \supset(5.2)$.

- In the same way, the uni-modal constraint of Formula (4.7) on the existence of $k$ distinct percepts is a particular instance of the corresponding multi-modal Formula (5.7) with $\pi_{R}=\perp$ such that $\models T((4.7)) \supset(5.7)$.

- The translation of uni-modal constraint on anchor fluents of Formula (4.10) to the multimodal language is restricted to singletons of percept identifiers, while both Formula (4.10) and Formula (5.10) implement the same requirement on anchors in the initial situation. Hence $\models T((4.10)) \supset(5.10)$. 
- Translating the uni-modal constraint of Formula (4.11) on initial anchor fluents yields the corresponding multi-modal Formula (5.11) such that $\models T((4.11)) \equiv(5.11)$ holds.

- The uni-modal precondition axiom of the epistemic symbol anchoring action AnchorU of Formula (4.17) is semantically equivalent to the corresponding multi-modal version of action Anchor $M$ defined in Formula (5.22). Hence, $\models T((4.17)) \equiv(5.22)$ holds.

- As will be described in more detail below, the methods of determining a fresh object symbol within the uni-modal and multi-modal versions of the symbol anchoring functionality New differ significantly. In particular, the uni-modal New uses the fluent Offset while the multimodal New does not. Thus, the requirement of Formula (4.31) becomes superfluous in the multi-modal approach such that $\models T((4.31)) \supset \top$.

Now, the main symbol anchoring Axiom (4.15) is the only one of the set $\Sigma_{u m s a}$ of uni-modal axioms, which a relation with the corresponding multi-modal axioms remains to be shown for according to our claim of the theorem at hand.

The Formula (5.36) stands for an intermediate step of this proof and represents the multi-modal symbol anchoring Axiom (5.23) restricted to uni-modal perception (cf. page 43). While multiple percepts may constitute a block of associated percepts originating from the same physical object in the case of multi-modal perception, only a single percept originates from an individual physical object in the uni-modal case. Limiting the multi-modal approach to uni-modal perception means that the following constraint in the multi-modal language has to hold true.

$$
P\left(\pi, o, \Gamma, \pi_{R}, s\right) \supset \operatorname{PBlock}(\{\pi\}, s)
$$

Accordingly, restricting our multi-modal approach to uni-modal perception infers that blocks of associated percepts are singletons only. We will return to this thread soon.

Next, we are going to restructure the uni-modal symbol anchoring Axiom (4.15) and its components into a semantically equivalent form. The resulting formula will allow us to show the logical relation to the multi-modal Axiom (5.23). The Formula (4.18) defining the uni-modal predicate $C C$ may be rewritten to a semantically equivalent form where the parameter "object category" $o$ is omitted and the construction of "minus" fluent $f^{-}$and "plus" fluent $f^{+}$is moved inside the restructured uni-modal functionalities $\Delta_{u T}^{\prime}, \Delta_{u F}^{\prime}$ and $\Delta_{u N}^{\prime}{ }^{5}$ The uni-modal predicate $C C^{\prime}$ implements the so adapted predicate $C C$ as follows.

$$
C C^{\prime}: \mathcal{X} \cup \text { PERCID } \times \text { FLUENT } \times \text { FLUENT } \times \text { SIT } \times \text { STATE }
$$

$$
\begin{aligned}
& C C^{\prime}\left(\pi_{P}, f^{-}, f^{+}, s, z\right) \equiv \\
& \quad(\exists x)\left(\Delta_{u T}^{\prime}\left(x, \pi_{P}, f^{-}, f^{+}, s, z\right) \vee \Delta_{u F}^{\prime}\left(x, \pi_{P}, f^{-}, f^{+}, s, z\right) \vee \Delta_{u N}^{\prime}\left(x, \pi_{P}, f^{-}, f^{+}, s, z\right)\right)
\end{aligned}
$$

The parameter $o$ : OBJCAT may be omitted from the original, uni-modal predicate $C C$ as it is bound and instantiated outside of $C C$ in the Axiom (4.15). The adapted functionalities $\Delta_{u T}^{\prime}$, $\Delta_{u F}^{\prime}$ and $\Delta_{u N}^{\prime}$ can determine the object category in question themselves easily and uniquely given the percept identifier $\pi_{P}$. The very same holds for the parameter $\Gamma$ : PERCSIG of the original predicate $C C$, which we omitted in the definition of $C C^{\prime}$, too. Thus, these structural modifications do not change the semantics of the uni-modal symbol anchoring Axioms (4.15), such that $=C C\left(\pi, o, \Gamma, n, f^{-}, f^{+}, s, z\right) \equiv C C^{\prime}\left(\pi, f^{-}, f^{+}, s, z\right)$. The disjunction of Formula (5.45) already resembles the disjunction of functionalities in the multi-modal Axiom (5.23).

Incorporating the above-mentioned modifications in the uni-modal functionalities Track and Find results in the following, adapted uni-modal macros $\Delta_{u T}^{\prime}$ and $\Delta_{u F}^{\prime}$, respectively. The modifications of functionality New will follow below.

\footnotetext{
${ }^{5}$ Henceforth, the subscripts $u$ and $m$ are used to indicate the uni-modal and the multi-modal versions of the macro at hand, respectively.
} 


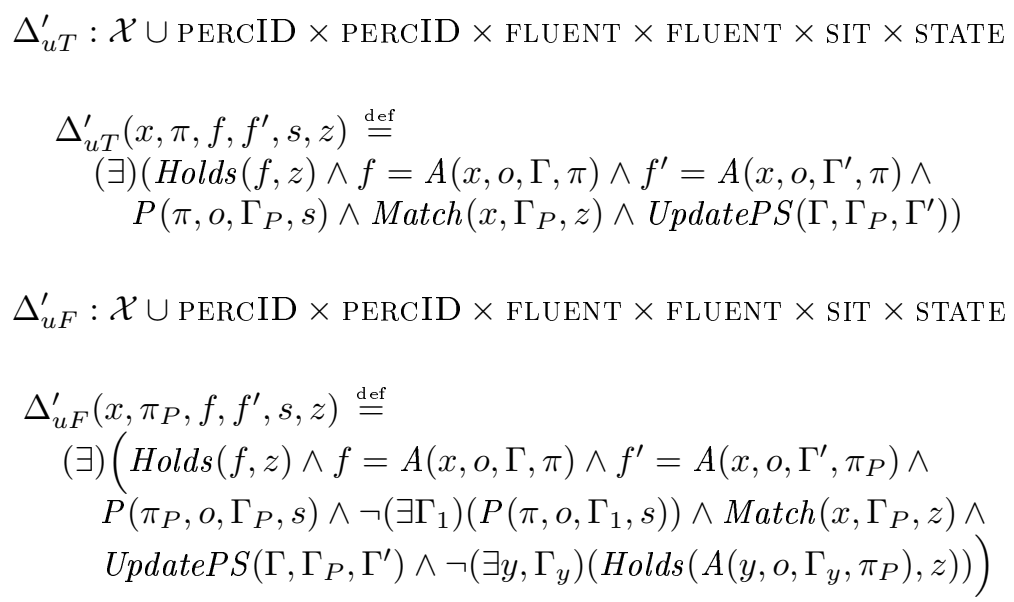

Next, we are going to replace the uni-modal method of determining fresh object symbols inside the functionality New with a simplified version, which is derived from the multi-modal New. The method of the multi-modal functionality New is to determine the maximum percept identifier of the considered block $\Pi$ of associated percepts and to use it for the fresh object symbol $x$, i. e., $x=\max \Pi$ (cf. Formula (5.30)). As sketched in Figure 5.3, this proof includes an intermediate step where the multi-modal symbol anchoring axiom is restricted to uni-modal perception (cf. Formula (5.36)). Due to limiting the multi-modal functionality New to uni-modal perception according to the Formula (5.44), the fresh object symbol simply equals the percept identifier, because the maximum percept identifier of a singleton $\Pi=\{\pi\}$ is the singleton's element $\pi$ itself with $\pi \in \mathbb{N}$.

Breaking up the uni-modal macros $\Delta_{N}$ and NewSymbol, the following subformula implements the functionality New as part of the predicate $C C$ in accordance with the uni-modal symbol anchoring Axiom (4.15):

$$
\begin{gathered}
(\forall)\left(\exists x, \Gamma_{P}, f^{-}, f^{+}\right)\left(P\left(\pi_{P}, o, \Gamma_{P}, s\right) \wedge \neg(\exists y, \Gamma)\left(\operatorname{Holds}\left(A\left(y, o, \Gamma, \pi_{P}\right), z\right)\right) \wedge\right. \\
\left.(\exists t)(\operatorname{Holds}(\operatorname{Offset}(t), z) \wedge x=t+n) \wedge f^{-}=\emptyset \wedge f^{+}=A\left(x, o, \Gamma_{P}, \pi_{P}\right)\right)
\end{gathered}
$$

Herein, replacing the method of determining fresh object symbols with the simplified, multi-modal method as described above results in the following subformula.

$$
\begin{gathered}
(\forall)\left(\exists x, \Gamma_{P}, f^{-}, f^{+}\right)\left(P\left(\pi_{P}, o, \Gamma_{P}, s\right) \wedge \neg(\exists y, \Gamma)\left(\operatorname{Holds}\left(A\left(y, o, \Gamma, \pi_{P}\right), z\right)\right) \wedge\right. \\
\left.x=\pi_{P} \wedge f^{-}=\emptyset \wedge f^{+}=A\left(x, o, \Gamma_{P}, \pi_{P}\right)\right)
\end{gathered}
$$

Both the uni-modal and the multi-modal methods achieve the same result, namely they determine a unique, fresh object symbol for a new percept. Moreover, the two Formulas (5.48) and (5.49) are semantically equivalent in the case of uni-modal perception. According to the new method of Formula (5.49), the predicate NewSymbol and the fluent Offset become obsolete as a consequence. The following, restructured macro $\Delta_{u N}^{\prime}$ implements the functionality New using the new method of Formula (5.49).

$$
\begin{gathered}
\Delta_{u N}^{\prime}: \text { PERCID } \times \text { PERCID } \times \text { FLUENT } \times \text { FLUENT } \times \text { SIT } \times \text { STATE } \\
\Delta_{u N}^{\prime}\left(x, \pi_{P}, f, f^{\prime}, s, z\right) \stackrel{\text { def }}{=} \\
(\exists)\left(P\left(\pi_{P}, o, \Gamma_{P}, s\right) \wedge f=\emptyset \wedge f^{\prime}=A\left(x, o, \Gamma_{P}, \pi_{P}\right) \wedge\right. \\
\left.\neg(\exists y, \Gamma)\left(\text { Hold } s\left(A\left(y, o, \Gamma, \pi_{P}\right), z\right)\right) \wedge x=\pi_{P}\right)
\end{gathered}
$$


Similarly, we adapt the uni-modal symbol anchoring Axiom (4.15) accordingly, yielding the following axiom.

$$
\begin{aligned}
& \operatorname{Poss}(\operatorname{AnchorU}(o), s) \supset \\
& \left(\exists k, s^{\prime}, \pi_{1}, \ldots, \pi_{k}, \Gamma_{1}, \ldots, \Gamma_{k}\right)\left(s^{\prime}=\operatorname{Do}(\operatorname{AnchorU}(o), s) \wedge N o P(s)=k \wedge\right. \\
& \left(\forall z^{\prime}\right)\left(K \operatorname{State}\left(s^{\prime}, z^{\prime}\right) \equiv\left(\exists z, f_{1}^{-}, \ldots, f_{k}^{-}, f_{1}^{+}, \ldots, f_{k}^{+}\right)(\text {KState }(s, z) \wedge\right. \\
& P\left(\pi_{1}, o, \Gamma_{1}, s\right) \wedge \cdots \wedge P\left(\pi_{k}, o, \Gamma_{k}, s\right) \wedge \\
& \quad C C^{\prime}\left(\pi_{1}, f_{1}^{-}, f_{1}^{+}, s, z\right) \wedge \cdots \wedge C^{\prime}\left(\pi_{k}, f_{k}^{-}, f_{k}^{+}, s, z\right) \wedge \\
& \left.\left.\left.\quad \text { Consistent }\left(z^{\prime}\right) \wedge z^{\prime}=z-f_{1}^{-} \circ \cdots \circ f_{k}^{-}+f_{1}^{+} \circ \cdots \circ f_{k}^{+}\right)\right)\right)
\end{aligned}
$$

Since the reorganization of the structure does not alter the semantics of the uni-modal symbol anchoring Axiom (4.15) in comparison with the above, revised Axiom (5.51), the following holds true.

$$
\models(4.15) \equiv(5.51)
$$

This equivalence corresponds to the Step 1 of the Figure 5.3 showing the structure of this proof. There, the Formula (5.34) stands for the above Axiom (5.51).

According to the Formula (5.43), applying the transformation function $T$ to a well-formed formula of the uni-modal language preserves the semantics. Hence, we may apply $T$ to the above Axiom (5.51) such that

$$
\models T((5.51)) \equiv(5.51)
$$

holds yielding the Step 2 of the proof chain displayed in Figure 5.3.

Next, we will focus on the subsequent steps of this proof chain, which the Formulas (5.35) and (5.36) represent in the Figure 5.3. As described above, these two formulas represent intermediate steps of this proof, where the multi-modal symbol anchoring axiom is restricted to uni-modal perception. In the case of uni-modal perception, the conjunct starting with PBlock $(\Pi, s) \supset \ldots$ of the multi-modal Axiom (5.23) may be simplified to $P\left(\pi, o, \Gamma, \pi_{R}, s\right) \supset \ldots$ referring to individual percepts directly. Due to the assumption of Formula (5.7) saying that $k$ distinct percepts exist in any situation with $k=N o P(s)$, the individual percepts may be enumerated explicitly in the symbol anchoring axiom. Thus, the axiom

$$
\begin{aligned}
& \operatorname{Poss}(\operatorname{AnchorM}(o), s) \supset\left(\exists s^{\prime}\right)\left(s^{\prime}=\operatorname{Do}(\operatorname{AnchorM}(o), s) \wedge\right. \\
& \left(\forall z^{\prime}\right)\left(\operatorname{KState}\left(s^{\prime}, z^{\prime}\right) \equiv\left(\exists z, \vartheta^{-}, \vartheta^{+}, \vartheta\right)(\operatorname{KState}(s, z) \wedge\right. \\
& N o P(s)=k \wedge P\left(\pi_{1}, o, \Gamma_{1}, \perp, s\right) \wedge \ldots \wedge P\left(\pi_{k}, o, \Gamma_{k}, \perp, s\right) \wedge \\
& {\left[P\left(\pi_{P}, o, \Gamma_{P}, \perp, s\right) \wedge \Pi_{P}=\left\{\pi_{P}\right\} \supset\left(\exists x, f^{-}, f^{+}\right)\left(\operatorname{Holds}\left(C\left(x, \Pi_{P}, f^{-}, f^{+}\right), \vartheta\right) \wedge\right.\right.} \\
& \left.\left.\left[\Delta_{T}\left(x, \Pi_{P}, f^{-}, f^{+}, s, z\right) \vee \Delta_{F}\left(x, \Pi_{P}, f^{-}, f^{+}, s, z\right) \vee \Delta_{N}\left(x, \Pi_{P}, f^{-}, f^{+}, s, z\right)\right]\right)\right] \wedge \\
& {\left[\operatorname{Holds}(f, \vartheta) \supset\left(\exists x, \Pi_{P}, f^{-}, f^{+}\right)\left(f=C\left(x, \Pi_{P}, f^{-}, f^{+}\right) \wedge \operatorname{PBlock}\left(\Pi_{P}, s\right)\right)\right] \wedge} \\
& \operatorname{Holds}\left(C\left(x_{1}, \Pi_{P}, f_{1}^{-}, f_{1}^{+}\right), \vartheta\right) \wedge \operatorname{Holds}\left(C\left(x_{2}, \Pi_{P}, f_{2}^{-}, f_{2}^{+}\right), \vartheta\right) \\
& \left.\supset x_{1}=x_{2} \wedge f_{1}^{-}=f_{2}^{-} \wedge f_{1}^{+}=f_{2}^{+}\right] \wedge \\
& {\left[\operatorname{Holds}\left(C\left(x, \Pi_{P 1}, f_{1}^{-}, f_{1}^{+}\right), \vartheta\right) \wedge \operatorname{Holds}\left(C\left(x, \Pi_{P 2}, f_{2}^{-}, f_{2}^{+}\right), \vartheta\right)\right.} \\
& \left.\supset \Pi_{P 1}=\Pi_{P 2} \wedge f_{1}^{-}=f_{2}^{-} \wedge f_{1}^{+}=f_{2}^{+}\right] \wedge \\
& {\left[\operatorname{Holds}\left(f^{+}, \vartheta^{+}\right) \equiv\left(\exists x, f^{-}, \Pi_{P}\right)\left(\operatorname{Holds}\left(C\left(x, \Pi_{P}, f^{-}, f^{+}\right), \vartheta\right)\right)\right] \wedge} \\
& {\left[\operatorname{Holds}\left(f^{-}, \vartheta^{-}\right) \equiv\left(\exists x, f^{+}, \Pi_{P}\right)\left(\operatorname{Holds}\left(C\left(x, \Pi_{P}, f^{-}, f^{+}\right), \vartheta\right) \wedge f^{-} \neq \emptyset\right)\right] \wedge} \\
& \left.\left.\left.z^{\prime}=z-\vartheta^{-}+\vartheta^{+}\right)\right)\right)
\end{aligned}
$$

follows from the multi-modal symbol anchoring Axiom (5.23) and the domain Constraint (5.44) representing uni-modal perception, such that 


$$
\{(5.23),(5.44)\} \models(5.52)
$$

Indicated by the dots between the percept predicates similar to the uni-modal symbol anchoring Axiom (4.15), the above Formula (5.52) implements a schematic formula depending on the number $k$ of percepts. Since it is the particular instances

$$
P\left(\pi_{1}, o, \Gamma_{1}, \perp, s\right) \wedge \ldots \wedge P\left(\pi_{k}, o, \Gamma_{k}, \perp, s\right)
$$

of the percept predicate which make the antecedent $P\left(\pi_{P}, o, \Gamma_{P}, \perp, s\right) \wedge \Pi_{P}=\left\{\pi_{P}\right\}$ of the implication of Formula (5.52) true, the Formula (5.52) may be transformed into the following equivalent formula. ${ }^{6}$

$$
\begin{aligned}
& \operatorname{Poss}(\operatorname{AnchorM}(o), s) \supset\left(\exists s^{\prime}\right)\left(s^{\prime}=\operatorname{Do}(\operatorname{AnchorM}(o), s) \wedge\right. \\
& \left(\forall z^{\prime}\right)\left(\operatorname{KState}\left(s^{\prime}, z^{\prime}\right) \equiv(\exists)(\operatorname{KState}(s, z) \wedge\right. \\
& N o P(s)=k \wedge P\left(\pi_{1}, o, \Gamma_{1}, \perp, s\right) \wedge \ldots \wedge P\left(\pi_{k}, o, \Gamma_{k}, \perp, s\right) \wedge \\
& {\left[P\left(\pi_{1}, o, \Gamma_{1}, \perp, s\right) \wedge \operatorname{Holds}\left(C\left(x_{1}, \Pi_{1}, f_{1}^{-}, f_{1}^{+}\right), \vartheta\right) \wedge \Pi_{1}=\left\{\pi_{1}\right\} \wedge\right.} \\
& \left.\left[\Delta_{T}\left(x_{1}, \Pi_{1}, f_{1}^{-}, f_{1}^{+}, s, z\right) \vee \Delta_{F}\left(x_{1}, \Pi_{1}, f_{1}^{-}, f_{1}^{+}, s, z\right) \vee \Delta_{N}\left(x_{1}, \Pi_{1}, f_{1}^{-}, f_{1}^{+}, s, z\right)\right]\right] \\
& \wedge \ldots \wedge \\
& {\left[P\left(\pi_{k}, o, \Gamma_{k}, \perp, s\right) \wedge \operatorname{Holds}\left(C\left(x_{k}, \Pi_{k}, f_{k}^{-}, f_{k}^{+}\right), \vartheta\right) \wedge \Pi_{k}=\left\{\pi_{k}\right\} \wedge\right.} \\
& \left.\left[\Delta_{T}\left(x_{k}, \Pi_{k}, f_{k}^{-}, f_{k}^{+}, s, z\right) \vee \Delta_{F}\left(x_{k}, \Pi_{k}, f_{k}^{-}, f_{k}^{+}, s, z\right) \vee \Delta_{N}\left(x_{k}, \Pi_{k}, f_{k}^{-}, f_{k}^{+}, s, z\right)\right]\right] \wedge \\
& {\left[\operatorname{Holds}(f, \vartheta) \supset(\exists)\left(f=C\left(x,\left\{\pi_{P}\right\}, f^{-}, f^{+}\right) \wedge P\left(\pi_{P}, o, \Gamma, \perp, s\right)\right)\right] \wedge} \\
& \operatorname{Holds}\left(C\left(x_{1}, \Pi_{P}, f_{1}^{-}, f_{1}^{+}\right), \vartheta\right) \wedge \operatorname{Holds}\left(C\left(x_{2}, \Pi_{P}, f_{2}^{-}, f_{2}^{+}\right), \vartheta\right) \\
& \left.\supset x_{1}=x_{2} \wedge f_{1}^{-}=f_{2}^{-} \wedge f_{1}^{+}=f_{2}^{+}\right] \wedge \\
& {\left[\operatorname{Holds}\left(C\left(x, \Pi_{P 1}, f_{1}^{-}, f_{1}^{+}\right), \vartheta\right) \wedge \operatorname{Holds}\left(C\left(x, \Pi_{P 2}, f_{2}^{-}, f_{2}^{+}\right), \vartheta\right)\right.} \\
& \left.\supset \Pi_{P 1}=\Pi_{P 2} \wedge f_{1}^{-}=f_{2}^{-} \wedge f_{1}^{+}=f_{2}^{+}\right] \wedge \\
& {\left[\operatorname{Holds}\left(f^{+}, \vartheta^{+}\right) \equiv\left(\exists x, f^{-}, \Pi_{P}\right)\left(\operatorname{Holds}\left(C\left(x, \Pi_{P}, f^{-}, f^{+}\right), \vartheta\right)\right)\right] \wedge} \\
& {\left[\operatorname{Holds}\left(f^{-}, \vartheta^{-}\right) \equiv\left(\exists x, f^{+}, \Pi_{P}\right)\left(\operatorname{Holds}\left(C\left(x, \Pi_{P}, f^{-}, f^{+}\right), \vartheta\right) \wedge f^{-} \neq \emptyset\right)\right] \wedge} \\
& \left.\left.z^{\prime}=z-\vartheta^{-}+\vartheta^{+}\right)\right) \text {) }
\end{aligned}
$$

The two axioms are equivalent, i. e.

$$
\models(5.52) \equiv(5.54)
$$

This equivalence holds, because: If some percept exists, the antecedent

$$
P\left(\pi_{P}, o, \Gamma_{P}, \perp, s\right) \wedge \Pi_{P}=\left\{\pi_{P}\right\}
$$

of the implication of Formula (5.52) is naturally true, such that it may be replaced with the conjunct $P\left(\pi_{i}, o, \Gamma_{i}, \perp, s\right) \wedge \ldots$ for $1 \leq i \leq k$ in Formula (5.54). Conversely, if no percept exists in a situation $s$, this antecedent holds false-hence, its implication becomes true automatically. However, in this case the conjunct

$$
\left[\operatorname{Holds}(f, \vartheta) \supset\left(\exists x, \Pi_{P}, f^{-}, f^{+}\right)\left(f=C\left(x, \Pi_{P}, f^{-}, f^{+}\right) \wedge \operatorname{PBlock}\left(\Pi_{P}, s\right)\right)\right]
$$

of Formula (5.52) makes sure that $\vartheta=\vartheta^{-}=\vartheta^{+}=\emptyset$, such that $z^{\prime}=z$, i. e., the Axiom (5.52) does not modify any symbol anchoring hypothesis. This is exactly how we designed the symbol anchoring axiom to work. The Axiom (5.54) has the very same effect.

\footnotetext{
${ }^{6}$ We use the notation $\Pi_{i}=\left\{\pi_{i}\right\}$ as an abbreviation, even though it could be simply replaced.
} 
Since it suffices to enumerate the instances of the percept predicate just once, the Axiom (5.54) may be rewritten into the following, semantically equivalent form.

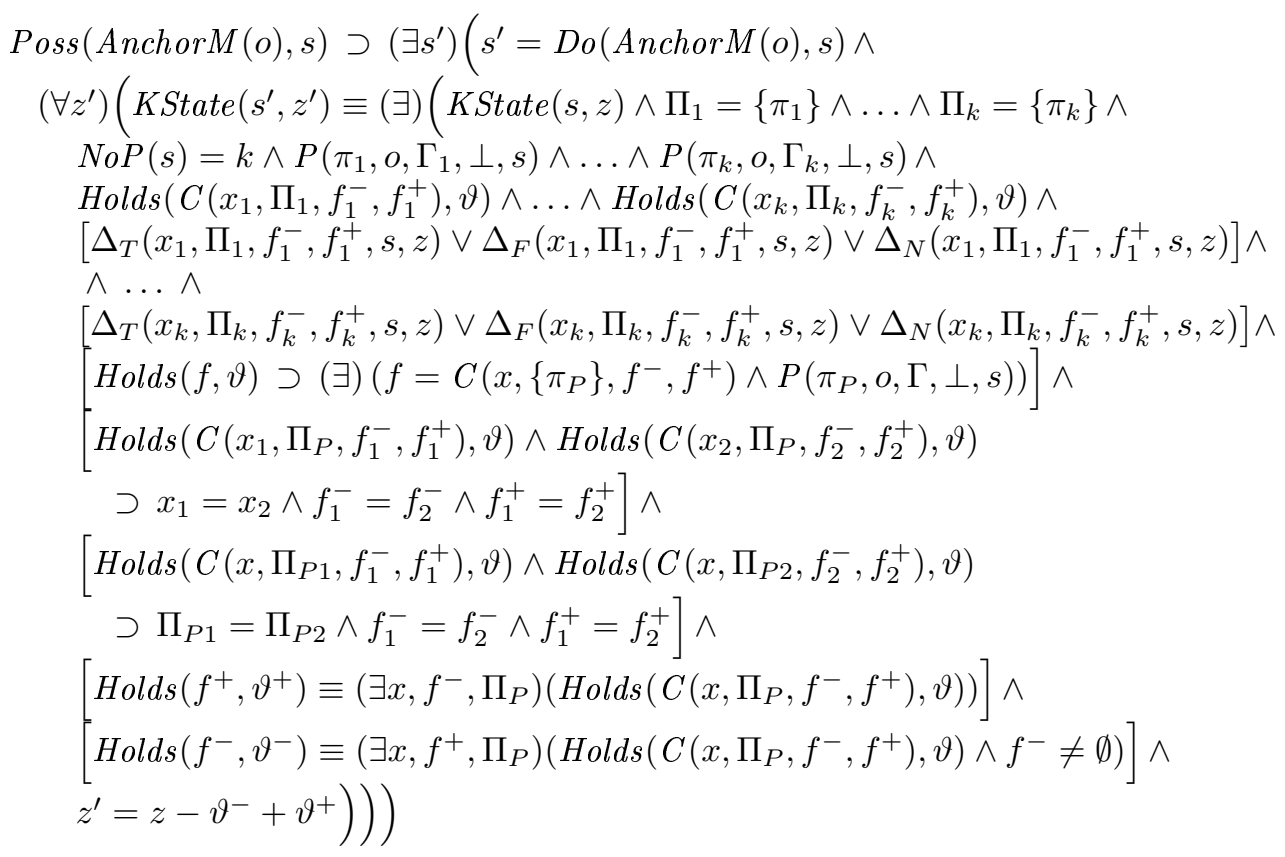

with

$$
\models(5.54) \equiv(5.56)
$$

Note that the existentially quantified predicates PFusion and AnchorUpdate-as employed inside the functionalities $\Delta_{T}, \Delta_{F}$ and $\Delta_{N}$ - are designed deterministically given an object symbol and a percept identifier. From this fact, together with the consistency constraints on the state term $\vartheta$ in Formula (5.54), it follows for the above symbol anchoring Axiom (5.56):

$$
\begin{gathered}
\vartheta=C\left(x_{1},\left\{\pi_{1}\right\}, f_{1}^{-}, f_{1}^{+}\right) \circ \ldots \circ C\left(x_{k},\left\{\pi_{k}\right\}, f_{k}^{-}, f_{k}^{+}\right) \\
\vartheta^{+}=f_{1}^{+} \circ \ldots \circ f_{k}^{+} \\
\vartheta^{-}=f_{1}^{-} \circ \ldots \circ f_{k}^{-}
\end{gathered}
$$

Recall the proof of Section 5.6.1 that the multi-modal symbol anchoring Axiom (5.23) only yields consistent successor hypotheses given consistent original hypotheses. This result allows to replace the consistency constraints inside Formula (5.56) with the predicate Consistent. Simultaneously, we may itemize the components of $\vartheta^{-}=f_{1}^{-} \circ \ldots \circ f_{k}^{-}$and of $\vartheta^{+}=f_{1}^{+} \circ \ldots \circ f_{k}^{+}$explicitly yielding the following formula

$$
\begin{aligned}
\operatorname{Poss}(\operatorname{AnchorM}(o), s) \supset\left(\exists s^{\prime}\right)\left(s^{\prime}=\operatorname{Do}(\operatorname{Anchor} M(o), s) \wedge\right. \\
\left(\forall z^{\prime}\right)\left(\text { KState } ( s ^ { \prime } , z ^ { \prime } ) \equiv ( \exists ) \left(\text { KState }(s, z) \wedge \Pi_{1}=\left\{\pi_{1}\right\} \wedge \ldots \wedge \Pi_{k}=\left\{\pi_{k}\right\} \wedge\right.\right. \\
\quad N o P(s)=k \wedge P\left(\pi_{1}, o, \Gamma_{1}, \perp, s\right) \wedge \ldots \wedge P\left(\pi_{k}, o, \Gamma_{k}, \perp, s\right) \wedge \\
\quad\left[\Delta_{T}\left(x_{1}, \Pi_{1}, f_{1}^{-}, f_{1}^{+}, s, z\right) \vee \Delta_{F}\left(x_{1}, \Pi_{1}, f_{1}^{-}, f_{1}^{+}, s, z\right) \vee \Delta_{N}\left(x_{1}, \Pi_{1}, f_{1}^{-}, f_{1}^{+}, s, z\right)\right] \\
\quad \wedge \ldots \wedge \\
\quad\left[\Delta_{T}\left(x_{k}, \Pi_{k}, f_{k}^{-}, f_{k}^{+}, s, z\right) \vee \Delta_{F}\left(x_{k}, \Pi_{k}, f_{k}^{-}, f_{k}^{+}, s, z\right) \vee \Delta_{N}\left(x_{k}, \Pi_{k}, f_{k}^{-}, f_{k}^{+}, s, z\right)\right] \\
\quad \text { Consistent }\left(z^{\prime}\right) \wedge \\
\left.\left.\left.\quad z^{\prime}=z-f_{1}^{-} \circ \ldots \circ f_{k}^{-}+f_{1}^{+} \circ \ldots \circ f_{k}^{+}\right)\right)\right)
\end{aligned}
$$

which is equivalent to Axiom (5.56) such that

$$
\models(5.56) \equiv(5.58)
$$


Next, we are going to discuss the individual components of the above Formula (5.58) regarding its relation with the Axiom (5.51). Recall that the latter formula is a restructured, but semantically equivalent version of the original, uni-modal symbol anchoring Axiom (4.15). Given the assumption of Formula (5.7) about the existence of $k$ distinct percepts in any situation, and limiting the multi-modal symbol anchoring axiom to uni-modal perception, we arrived at the Axiom (5.58) above. Here, blocks of associated percepts become singletons, such that the multi-modal macros $\Delta_{T}, \Delta_{F}$ and $\Delta_{N}$ take singletons of percepts in their second parameter (cf. Formula (5.44)). Therefore, the antecedent $\pi_{P} \in \Pi_{P}$ of the implication inside the definitions of these macros always holds true if the number of available percepts is more than zero. ${ }^{7}$ Each macro definition verifies the validity of the given percept identifier itself again. Therefore, this implication of each multi-modal macro definition $\Delta_{T}, \Delta_{F}$ and $\Delta_{N}$ may be transformed into a conjunction in the case of uni-modal perception. This way we derived the following, modified macro definitions from the original Definitions (5.25), (5.29) and (5.30) of the multi-modal symbol anchoring functionalities Track, Find and New, respectively, given the restriction to uni-modal perception. $^{8}$

$$
\begin{aligned}
& \Delta_{m T}^{u}: \mathcal{X} \cup \text { PERCID } \times \mathcal{P}(\text { PERCID }) \times \text { FLUENT } \times \text { FLUENT } \times \text { SIT } \times \text { STATE } \\
& \Delta_{m T}^{u}\left(x, \Pi_{P}, f, f^{\prime}, s, z\right) \stackrel{\text { def }}{=} \\
& \text { ( } \exists)\left(\operatorname{Holds}(f, z) \wedge f=A\left(x, o, \Gamma_{A}, \Pi_{A}\right) \wedge f^{\prime}=A\left(x, o, \Gamma^{\prime}, \Pi^{\prime}\right) \wedge \Pi_{P}=\left\{\pi_{P}\right\} \wedge\right. \\
& P\left(\pi_{P}, o, \Gamma_{P}, \pi_{R}, s\right) \wedge P \operatorname{Known}\left(\Pi_{A}, \pi_{P}, \pi_{R}\right) \wedge \operatorname{Match}\left(x, \Gamma_{P}, z\right) \wedge \\
& \text { AnchorUpdate } \left.\left(o, \Gamma_{A}, \Pi_{A}, \Pi_{P}, \Gamma^{\prime}, \Pi^{\prime}, s\right)\right) \\
& \Delta_{m F}^{u}: \mathcal{X} \cup \text { PERCID } \times \mathcal{P}(\text { PERCID }) \times \text { FLUENT } \times \text { FLUENT } \times \text { SIT } \times \text { STATE } \\
& \Delta_{m F}^{u}\left(x, \Pi_{P}, f, f^{\prime}, s, z\right) \stackrel{\text { def }}{=} \\
& (\exists)\left(\operatorname{Holds}(f, z) \wedge f=A\left(x, o, \Gamma_{A}, \Pi_{A}\right) \wedge f^{\prime}=A\left(x, o, \Gamma^{\prime}, \Pi^{\prime}\right) \wedge \Pi_{P}=\left\{\pi_{P}\right\} \wedge\right. \\
& \neg\left(\exists \pi_{1}, o_{1}, \Gamma_{1}, \pi_{R 1}\right)\left(P\left(\pi_{1}, o_{1}, \Gamma_{1}, \pi_{R 1}, s\right) \wedge P \operatorname{Known}\left(\Pi_{A}, \pi_{1}, \pi_{R 1}\right)\right) \wedge \\
& P\left(\pi_{P}, o, \Gamma_{P}, \pi_{R}, s\right) \wedge \operatorname{Match}\left(x, \Gamma_{P}, z\right) \wedge \text { AnchorUpdate }\left(o, \Gamma_{A}, \Pi_{A}, \Pi_{P}, \Gamma^{\prime}, \Pi^{\prime}, s\right) \wedge \\
& \left.\neg\left(\exists y, o_{y}, \Gamma_{y}, \Pi_{y}\right)\left(\operatorname{Holds}\left(A\left(y, o_{y}, \Gamma_{y}, \Pi_{y}\right), z\right) \wedge P \operatorname{Known}\left(\Pi_{y}, \pi_{P}, \pi_{R}\right)\right)\right) \\
& \Delta_{m N}^{u}: \text { PERCID } \times \mathcal{P}(\text { PERCID }) \times \text { FLUENT } \times \text { FLUENT } \times \text { SIT } \times \text { STATE } \\
& \Delta_{m N}^{u}\left(x, \Pi_{P}, f, f^{\prime}, s, z\right) \stackrel{\text { def }}{=} \\
& \text { ( } \exists)\left(f=\emptyset \wedge f^{\prime}=A\left(x, o, \Gamma^{\prime}, \Pi_{P}\right) \wedge \Pi_{P}=\left\{\pi_{P}\right\} \wedge x=\pi_{P} \wedge\right. \\
& P\left(\pi_{P}, o, \Gamma_{P}, \pi_{R}, s\right) \wedge P F u s i o n\left(o, \Pi_{P}, \Gamma^{\prime}, s\right) \wedge \\
& \left.\neg\left(\exists y, o_{y}, \Gamma_{y}, \Pi_{y}\right)\left(\operatorname{Holds}\left(A\left(y, o_{y}, \Gamma_{y}, \Pi_{y}\right), z\right) \wedge \operatorname{PKnown}\left(\Pi_{y}, \pi_{P}, \pi_{R}\right)\right)\right)
\end{aligned}
$$

In the case of uni-modal perception, these multi-modal macros are equivalent to their original counterparts. Thus, we may replace the original macros of Axiom (5.58) with the above, restricted

\footnotetext{
${ }^{7}$ If no percepts are available, the $k$ conjuncts $\left[\Delta_{T}(\ldots) \vee \Delta_{F}(\ldots) \vee \Delta_{N}(\ldots)\right]$ of Axiom (5.58) vanish altogether, because the axiom is a schematic formula depending on the number of percepts.

${ }^{8}$ The superscript ${ }^{u}$ serves the purpose of indicating that the formula at hand is restricted to uni-modal perception in the sequel.
} 
versions yielding the following axiom.

$$
\begin{aligned}
& \operatorname{Poss}(\operatorname{AnchorM}(o), s) \supset\left(\exists s^{\prime}\right)\left(s^{\prime}=\operatorname{Do}(\operatorname{AnchorM}(o), s) \wedge\right. \\
& \left(\forall z^{\prime}\right)\left(K \operatorname { K t a t e } ( s ^ { \prime } , z ^ { \prime } ) \equiv ( \exists ) \left(\text { KState }(s, z) \wedge \Pi_{1}=\left\{\pi_{1}\right\} \wedge \ldots \wedge \Pi_{k}=\left\{\pi_{k}\right\} \wedge\right.\right. \\
& N o P(s)=k \wedge P\left(\pi_{1}, o, \Gamma_{1}, \perp, s\right) \wedge \ldots \wedge P\left(\pi_{k}, o, \Gamma_{k}, \perp, s\right) \wedge \\
& {\left[\Delta_{m T}^{u}\left(x_{1}, \Pi_{1}, f_{1}^{-}, f_{1}^{+}, s, z\right) \vee \Delta_{m F}^{u}\left(x_{1}, \Pi_{1}, f_{1}^{-}, f_{1}^{+}, s, z\right) \vee \Delta_{m N}^{u}\left(x_{1}, \Pi_{1}, f_{1}^{-}, f_{1}^{+}, s, z\right)\right]} \\
& \wedge \ldots \wedge \\
& {\left[\Delta_{m T}^{u}\left(x_{k}, \Pi_{k}, f_{k}^{-}, f_{k}^{+}, s, z\right) \vee \Delta_{m F}^{u}\left(x_{k}, \Pi_{k}, f_{k}^{-}, f_{k}^{+}, s, z\right) \vee \Delta_{m N}^{u}\left(x_{k}, \Pi_{k}, f_{k}^{-}, f_{k}^{+}, s, z\right)\right]} \\
& \text { Consistent }\left(z^{\prime}\right) \wedge \\
& \left.\left.\left.z^{\prime}=z-f_{1}^{-} \circ \ldots \circ f_{k}^{-}+f_{1}^{+} \circ \ldots \circ f_{k}^{+}\right)\right)\right)
\end{aligned}
$$

Both being restricted to uni-modal perception, the Axioms (5.58) and (5.63) are semantically equivalent, i.e.

$$
\models(5.58) \equiv(5.63)
$$

Next, we will examine the individual components of the above multi-modal Axiom (5.63) and their relation to the corresponding uni-modal components. Given the restriction to uni-modal perception of the Axiom (5.63), the predicate PKnown may be replaced with the term $\pi \in \Pi_{P}$. Hence, the uni-modal requirement

$$
\operatorname{Holds}(A(x, o, \Gamma,\{\pi\}), z) \wedge P\left(\pi, o, \Gamma_{P}, \perp, s\right)
$$

of $T\left(\Delta_{u T}^{\prime}\right)$ (transformation function $T$ applied to Formula (5.46)) and of $T\left(\Delta_{u F}^{\prime}\right)$ implies the corresponding multi-modal requirement

$$
\operatorname{Holds}\left(A\left(x, o, \Gamma, \Pi_{A}\right), z\right) \wedge P\left(\pi, o, \Gamma_{P}, \perp, s\right) \wedge \pi \in \Pi_{A}
$$

of $\Delta_{m T}^{u}$ and of $\Delta_{m F}^{u}$. Similarly, the uni-modal requirement

$$
P\left(\pi_{P}, o, \Gamma_{P}, \perp, s\right) \wedge \neg\left(\exists y, \Gamma_{y}\right)\left(\operatorname{Holds}\left(A\left(y, o, \Gamma_{y},\left\{\pi_{P}\right\}\right), z\right)\right)
$$

of $T\left(\Delta_{u F}^{\prime}\right)$ and of $T\left(\Delta_{u N}^{\prime}\right)$ implies the corresponding multi-modal requirement

$$
P\left(\pi_{P}, o, \Gamma_{P}, \perp, s\right) \wedge \neg\left(\exists y, o_{y}, \Gamma_{y}, \Pi_{y}\right)\left(\operatorname{Holds}\left(A\left(y, o_{y}, \Gamma_{y}, \Pi_{y}\right), z\right) \wedge \pi_{P} \in \Pi_{y}\right)
$$

of $\Delta_{m F}^{u}$ and of $\Delta_{m N}^{u}$. The multi-modal predicate AnchorUpdate-as used in the definitions of functionalities Track and Find-generalizes the uni-modal predicate UpdatePS. In addition to what UpdatePS deduces, the predicate AnchorUpdate determines the successor set $\Pi^{\prime}$ of associated percepts of the anchor fluent. Both predicates implement the conditions and the computation of new attribute-value pairs to become part of the new perceptual estimate. In both predicates AnchorUpdate and UpdatePS, previous perceptual estimates of static attributes as well as all kinds of attributes of measured perceptual values constrain the successor estimates in the very same way. The uni-modal condition

$$
\begin{aligned}
& \text { AttrTypeStatic }(o, a) \wedge\left(\gamma \in\left(\Gamma_{A} \cup \Gamma_{P}\right) \wedge \gamma=(a,(b, t)) \supset b \leq b^{\prime} \wedge t \geq t^{\prime}\right) \\
& \left.\vee \neg \operatorname{AttrTypeStatic}(o, a) \wedge(\exists \gamma)\left(\gamma=\left(a,\left(b^{\prime}, t^{\prime}\right)\right) \wedge \gamma \in \Gamma_{P}\right)\right)
\end{aligned}
$$

on $b^{\prime}$ and $t^{\prime}$ inside of predicate UpdatePS (Formula (4.25)) implies the corresponding multimodal condition inside of predicate AnchorUpdate (cf. Formula (5.28)):

$$
\begin{aligned}
& \left(\gamma \in \Gamma_{A} \wedge \gamma=(a,(b, t)) \wedge \operatorname{AttrTypeStatic}(o, a) \supset b \leq b^{\prime} \wedge t \geq t^{\prime}\right) \wedge \\
& \left(\pi_{P} \in \Pi_{P} \wedge P\left(\pi_{P}, o, \Gamma_{P}, \pi_{R}, s\right) \wedge \gamma_{P} \in \Gamma_{P} \wedge \gamma_{P}=\left(a,\left(b_{P}, t_{P}\right)\right)\right. \\
& \left.\quad \supset b_{P} \leq b^{\prime} \wedge t_{P} \geq t^{\prime}\right)
\end{aligned}
$$


This relation becomes more obvious in the case of uni-modal perception (cf. Formula (5.44)). Also, the remaining condition on the updated perceptual estimate of both predicates represents the same requirements in the case of uni-modal perception. In particular, the uni-modal condition

$$
\begin{aligned}
& \text { AttrTypeStatic }(o, a) \wedge \\
& \quad(\exists \gamma, b, t)\left(\gamma=(a,(b, t)) \wedge b=b^{\prime} \wedge \gamma \in\left(\Gamma_{A} \cup \Gamma_{P}\right)\right) \wedge \\
& \quad(\exists \gamma, b, t)\left(\gamma=(a,(b, t)) \wedge t=t^{\prime} \wedge \gamma \in\left(\Gamma_{A} \cup \Gamma_{P}\right)\right) \\
& \vee \neg \text { AttrTypeStatic }(o, a) \wedge(\exists \gamma)\left(\gamma=\left(a,\left(b^{\prime}, t^{\prime}\right)\right) \wedge \gamma \in \Gamma_{P}\right)
\end{aligned}
$$

on $b^{\prime}$ and $t^{\prime}$ inside of predicate UpdatePS (Formula (4.25)) implies the multi-modal subformula

$$
\begin{gathered}
(\exists \gamma, b, t)\left[\gamma=(a,(b, t)) \wedge b^{\prime}=b \wedge\left(\gamma \in \Gamma_{A} \wedge \operatorname{AttrTypeStatic}(o, a)\right.\right. \\
\left.\left.\vee\left(\exists \pi_{P}, \Gamma_{P}, \pi_{R}\right)\left(\pi_{P} \in \Pi_{P} \wedge P\left(\pi_{P}, o, \Gamma_{P}, \pi_{R}, s\right) \wedge \gamma \in \Gamma_{P}\right)\right)\right] \wedge \\
(\exists \gamma, b, t)\left[\gamma=(a,(b, t)) \wedge t^{\prime}=t \wedge\left(\gamma \in \Gamma_{A} \wedge \text { AttrTypeStatic }(o, a)\right.\right. \\
\left.\left.\vee \vee\left(\exists \pi_{P}, \Gamma_{P}, \pi_{R}\right)\left(\pi_{P} \in \Pi_{P} \wedge P\left(\pi_{P}, o, \Gamma_{P}, \pi_{R}, s\right) \wedge \gamma \in \Gamma_{P}\right)\right)\right]
\end{gathered}
$$

of predicate AnchorUpdate (Formula (5.28)) in case we restrict the multi-modal AnchorUpdate to uni-modal perception according to Formula (5.44). Thus, the conjunction of both conditions of the uni-modal predicate UpdatePS discussed above implies the conjunction of the corresponding conditions of the multi-modal predicate AnchorUpdate such that:

$$
\models\left(\exists \Gamma^{\prime}\right)\left(T\left(\text { UpdatePS }\left(\Gamma, \Gamma_{P}, \Gamma^{\prime}\right)\right)\right) \supset\left(\exists \Gamma^{\prime}, \Pi^{\prime}\right)\left(\text { AnchorUpdate }\left(o, \Gamma_{A}, \Pi_{A}, \Pi_{P}, \Gamma^{\prime}, \Pi^{\prime}, s\right)\right)
$$

Let us denote the uni-modal predicate Match (Formula (4.20)) and the corresponding multimodal version (Formula (5.27)) with Match $_{u}$ and Match $_{m}$, respectively. It is easy to see that both predicates are semantically equivalent if they share the same logical language, which is $\mathcal{L}_{m}$ in our case. Hence,

$$
\models T\left(\operatorname{Match}_{u}(x, \Gamma, z)\right) \equiv \operatorname{Match}_{m}(x, \Gamma, z)
$$

Altogether, the above shows that the uni-modal functionality Track (Formula (5.46)) implies the multi-modal Track (Formula (5.60)) under the restriction of uni-modal perception, i.e.,

$$
\models T\left(\Delta_{u T}^{\prime}\left(x, \pi, f^{-}, f^{+}, s, z\right)\right) \supset \Delta_{m T}^{u}\left(x,\{\pi\}, f^{-}, f^{+}, s, z\right)
$$

As for the symbol anchoring functionality Find, the uni-modal condition

$$
(\exists)\left(\operatorname{Holds}(f, z) \wedge f=A(x, o, \Gamma, \pi) \wedge \neg\left(\exists \Gamma_{1}\right)\left(P\left(\pi, o, \Gamma_{1}, s\right)\right)\right)
$$

of Formula (5.47) certainly implies the corresponding multi-modal condition of Formula (5.61):

$$
\begin{aligned}
(\exists) & \left(\operatorname{Holds}(f, z) \wedge f=A\left(x, o, \Gamma_{A}, \Pi_{A}\right) \wedge\right. \\
& \left.\neg\left(\exists \pi_{1}, o_{1}, \Gamma_{1}, \pi_{R 1}\right)\left(P\left(\pi_{1}, o_{1}, \Gamma_{1}, \pi_{R 1}, s\right) \wedge \operatorname{PKnown}\left(\Pi_{A}, \pi_{1}, \pi_{R 1}\right)\right)\right)
\end{aligned}
$$

in the case of uni-modal perception and given a common language, i. e., if we apply the transformation function $T$. Likewise, after applying the transformation function $T$ to the condition

$$
\begin{gathered}
(\exists)\left(\operatorname{Holds}(f, z) \wedge f=A(x, o, \Gamma, \pi) \wedge P\left(\pi_{P}, o, \Gamma_{P}, s\right) \wedge\right. \\
\left.\operatorname{Match}\left(x, \Gamma_{P}, z\right) \wedge \operatorname{UpdatePS}\left(\Gamma, \Gamma_{P}, \Gamma^{\prime}\right)\right)
\end{gathered}
$$

of the uni-modal definition of Find in Formula (5.47), this subformula logically implies the corresponding part of the multi-modal Formula (5.61) being

$$
\begin{gathered}
(\exists)\left(\operatorname{Holds}(f, z) \wedge f=A\left(x, o, \Gamma_{A}, \Pi_{A}\right) \wedge P\left(\pi_{P}, o, \Gamma_{P}, \pi_{R}, s\right) \wedge \operatorname{Match}\left(x, \Gamma_{P}, z\right) \wedge\right. \\
\left.\Pi_{P}=\left\{\pi_{P}\right\} \wedge \text { AnchorUpdate }\left(o, \Gamma_{A}, \Pi_{A}, \Pi_{P}, \Gamma^{\prime}, \Pi^{\prime}, s\right)\right)
\end{gathered}
$$


This relation becomes apparent if we take the above results regarding the predicates Match, UpdatePS and AnchorUpdate into account. Similarly, given a common language, the remaining part

$$
(\exists)\left(P\left(\pi_{P}, o, \Gamma_{P}, s\right) \wedge \neg\left(\exists y, \Gamma_{y}\right)\left(\operatorname{Holds}\left(A\left(y, o, \Gamma_{y}, \pi_{P}\right), z\right)\right)\right)
$$

of the definition of the uni-modal functionality Find of Formula (5.47) logically implies the corresponding, multi-modal condition

$$
(\exists)\left(P\left(\pi_{P}, o, \Gamma_{P}, \pi_{R}, s\right) \wedge \neg\left(\exists y, o_{y}, \Gamma_{y}, \Pi_{y}\right)\left(\operatorname{Holds}\left(A\left(y, o_{y}, \Gamma_{y}, \Pi_{y}\right), z\right) \wedge P \operatorname{Rnown}\left(\Pi_{y}, \pi_{P}, \pi_{R}\right)\right)\right)
$$

of Formula (5.61) in case of uni-modal perception. Apparently, this holds true because the transformation function $T$ yields a common representation and the above multi-modal formula is more general than the uni-modal one. Each of the above two pairs of conditions constitutes the unimodal and multi-modal definitions of functionality Find, respectively, in a single conjunction. Hence, the uni-modal macro Find of Formula (5.47) logically implies the multi-modal macro Find of Formula (5.61) in the limited case of uni-modal perception such that the following holds:

$$
\models T\left(\Delta_{u F}^{\prime}\left(x, \pi, f^{-}, f^{+}, s, z\right)\right) \supset \Delta_{m F}^{u}\left(x,\{\pi\}, f^{-}, f^{+}, s, z\right)
$$

Next, we are going to show that the uni-modal functionality New implies the multi-modal New. The multi-modal predicate PFusion is defined in Formula (5.31) as a specific instance of the predicate AnchorUpdate. The predicate PFusion combines the measured perceptual information in form of a given block of percepts into a single perceptual estimate. Due to the restriction to uni-modal perception, which causes blocks of associated percepts to become singleton sets only, the multi-modal functionality New calls the predicate PFusion with just a single percept to be fused. Hence, nothing is left to be done for the predicate PFusion, such that:

$$
P\left(\pi_{P}, o, \Gamma_{P}, \pi_{R}, s\right) \wedge \operatorname{PBlock}\left(\left\{\pi_{P}\right\}, s\right) \supset \operatorname{PFusion}\left(o,\left\{\pi_{P}\right\}, \Gamma_{P}, s\right)
$$

The remaining part of the macro $\Delta_{u N}^{\prime}$ is very similar to the functionalities Track and Find; therefore we refer to the above reasoning. Altogether this shows that the uni-modal symbol anchoring functionality New (Formula (5.50)) implies the multi-modal New (Formula (5.62)) in case of uni-modal perception, if they share the same representation, such that the following holds.

$$
T\left(\Delta_{u N}^{\prime}\left(x, \pi_{P}, f, f^{\prime}, s, z\right)\right) \supset \Delta_{m N}^{u}\left(x,\left\{\pi_{P}\right\}, f, f^{\prime}, s, z\right)
$$

This completes the analysis of the relation between the uni-modal symbol anchoring Axiom (5.51) and the multi-modal Axiom (5.63) with the following result.

$$
\models T((5.51)) \supset(5.63)
$$

This relation is represented by Step 3 in the Figure 5.3 above showing the structure of this proof. The Step 4 of this figure follows from the equivalences represented in Formulas (5.55), (5.57), (5.59) and (5.64).

According to Formula (5.53), we derived the Axiom (5.52) from the original multi-modal symbol anchoring Axiom (5.23) and the Formula (5.44) constraining the multi-modal approach to unimodal perception. Thus, the relation of Step 5 of Figure 5.3 holds as follows:

$$
\models(5.52) \supset(5.23)
$$

Now, all steps of Figure 5.3 have been shown, which completes the proof.

In other words, the uni-modal symbol anchoring approach presented in Chapter 4 may be rightfully considered a specific instance of the our multi-modal approach presented in Chapter 5 above. This way, the proven characteristics of the multi-modal approach described in the previous sections apply likewise to the uni-modal approach. 


\subsection{Summary and Conclusion}

In Chapter 5 above we presented our multi-modal approach to the symbol anchoring problem. Accounting for multiple, simultaneous modalities of perception, this approach generalizes our unimodal approach explicated in Chapter 4 and extends its limited applicability towards projects of state-of-the-art cognitive robotics, wherein typically multiple different kinds of sensor devices are employed in perceiving objects and features of the robot's environment. Multiple different sensing devices perceiving objects of the world also contribute to improving the robustness and precision of symbol anchoring, as more perceptual attributes yield further evidence which symbol anchoring relies on.

While the multi-modal symbol anchoring approach generalizes our uni-modal approach, they share the same objectives and fundamental concepts as described in Chapter 3. Thus, the multimodal approach of this chapter also satisfies all those functional requirements which the uni-modal approach accounts for. Our uni-modal approach has been evaluated in Chapter 4 against the requirements specified by Coradeschi and Saffiotti [2002] (cf. Section 2.3.4). However, we briefly analyse our multi-modal approach here, too. The first one of these requirements says that a symbol anchoring approach shall afford to specify the reference to an object by means of a symbolic description. This requirement is likewise accounted for in both our uni-modal approach and our multi-modal approach, which Section 3.7 explains. Next, the authors demanded that a symbol anchoring system shall implement the possibility to search for the appropriate object of the environment given a certain object symbol and some percepts measured by the recognition system, and then to establish a new correspondence accordingly. The uni-modal functionalities Find and New explicated in Sections 4.2.3 and 4.2.4 respectively, as well as and our multi-modal versions of Find and of New described in Sections 5.4.5 and 5.4.6 respectively, implement exactly this task. Furthermore, Coradeschi and Saffiotti [2002] specified that any approach shall be able to deal with ambiguity of symbol anchoring appropriately. We explained in Chapter 3 how various techniques take ambiguity into account in our approaches. In particular, our multi-modal approach maintains all plausible hypotheses of correspondences automatically, represents perceptual uncertainty and vague symbolic descriptions of objects, verifies the plausibility of hypotheses and eliminates inconsistent ones automatically if applicable. Moreover, the Section 4.3 proposed some techniques to prefer certain hypotheses over others. Next, a symbol anchoring approach is required to track an anchor, i.e., to update a correspondence between object symbol and the percepts representing the anchored, physical object. Our uni-modal functionality Track explicated in Section 4.2.2 and the multi-modal Track of Section 5.4.4 address this task explicitly. Finally, Coradeschi and Saffiotti [2002] specified that a symbol anchoring approach shall be able to reacquire a lost correspondence if the object reappeared after tracking has failed previously. We highlighted in Section 5.4.5 that our multi-modal functionality Find also accomplishes this task, and likewise does the uni-modal Find as described in Section 4.2.3.

The extended flexibility of our multi-modal symbol anchoring approach stems from considering sets of associated percepts - each of them representing the same object of the environment-for the correspondence between an object symbol and the referred physical object. Previously in the uni-modal approach, only a single percept was allowed to ground the correspondence of an object symbol on a physical object. Multiple independent percepts, which all have been actually measured from the same physical object, being provided by the multi-modal object recognition system individually, introduce an additional combinatorial problem if they are not indicated to refer to the same physical object. Therefore the object recognition system is asked to, but not necessarily has to, report which previous percept a new percept refers to in case they have been measured of the same physical object being tracked robustly. The percept predicate holds an additional parameter for communicating the reference percept for this purpose. Our symbol anchoring approach maintains the set of all previously corresponding percepts inside the anchor fluent facilitating to resolve the reference to some previous object.

Each time a symbol anchoring update is to be performed, our multi-modal symbol anchoring axiom accomplishes the following four main tasks. 
1. Given that percepts may refer to some previous percept in case of successful tracking, our approach determines the sets of associated percepts, each of the sets comprising only such percepts which refer to the same physical object. The symbol anchoring axiom is designed in a flexible way which accounts for an arbitrary number of percepts forming a set of associated percepts.

2. For each set of associated percepts, the possible correspondences with object symbols are determined verifying whether all the symbolic and perceptual information gathered so far match consistently including the latest measurements. Note that our approach automatically determines all possible correspondences for all available current percepts at once, yielding multiple hypotheses of correspondences in general.

3. For each possible correspondence between percepts and object symbols determined, the new perceptual information of the set of associated percepts and the perceptual estimate of the correspondence maintained in the anchor fluent are fused forming a new perceptual estimate. The sensor fusion method combines different perceptual attributes measured by different sensors into a single object model. Also, common subsets of perceptual attributes measured by different sensors may complement each other providing a single, up-to-date object model.

4. Lastly, the representation of each determined correspondence is updated with the new perceptual object model. In particular, the perceptual estimate and the set of corresponding percepts are maintained inside the anchor fluent of a correspondence.

The symbol anchoring axiom of the epistemic anchoring action implements the above tasks using three different symbol anchoring functionalities: Track, Find and New. Depending on the particular configuration of the correspondence under consideration, either functionality applies if the correspondence can be updated to the next situation. Each one of the functionalities Track, Find and New performs the above four tasks for the given correspondence in a certain, well-defined manner presupposing certain conditions to hold, which in the end achieves the objectives of symbol anchoring irrespective of the cardinality of the considered set of associated percepts. In particular, the latest percepts must agree with the symbolic information and the perceptual object model obtained so far about an object. On top of that, the symbol anchoring axiom verifies that each new successor hypothesis complies with the consistency constraints as a whole.

A new successor hypothesis must originate from some previous hypothesis, which the symbol anchoring axiom updates toward the successor situation. Conversely, a single previous hypothesis may result in zero, one or multiple valid successor hypotheses. No such successor hypothesis may be generated if some inconsistency of perceptual and symbolic information disallows it. In that case, the hypothesis thread ceases to exist in the successor situation, because our approach disregards inconsistent hypotheses automatically. All valid successor hypotheses are determined and generated automatically. The complexity of the resulting hypothesis space effectively is similar to the one of our uni-modal approach. On the one hand, dissociated percepts, which were not indicated to refer to the same physical object like other percepts, introduce additional, alternative hypotheses. On the other hand, multiple different sensor devices typically provide a rich perceptual image of the object comprising more details than a single kind of sensor could yield, reducing ambiguity in the first place.

Note that the same general concepts of our uni-modal symbol anchoring approach apply to our multi-modal approach, too. Since these concepts have been discussed in Chapter 4 already in detail, the reader is referred to the respective sections. In summary, to develop a general symbol anchoring approach which is independent of the application domain counts among our main objectives. Employing a particular calculus of predicate logic, the Fluent Calculus endows our approach with a high symbolic expressiveness for representing and reasoning about multiple symbol anchoring hypotheses and knowledge about objects of the robot's environment. Thanks to the purely symbolic nature we were able to design our multi-modal approach in accordance with certain important characteristics and to prove that the formulas presented above actually comply 
with these characteristics. For instance, given a consistent definition of the initial hypotheses of correspondences, our approach has been shown to generate valid, consistent successor hypotheses only. Furthermore, our approach guarantees to associate each percept with some object symbol such that no perceptual information is neglected.

Being based on the Fluent Calculus, our approach is fully integrated with the symbolic reasoning and knowledge representation system. The level of logical expressiveness available in our approach is unmatched by any other symbol anchoring approach to the best of our knowledge at time of writing. In comparison with other multi-modal approaches to the symbol anchoring problem, Fritsch et al. [2003] considered multi-modal perception for symbol anchoring, but disregarded the symbolic level altogether. Thus their approach neglects the essential capacity to identify the objects of interest which constitutes the main objective of symbol anchoring. Conversely, our approach computes and updates all possible hypotheses of correspondences automatically and takes all percepts into account for object identification. 


\section{Chapter 6}

\section{Conclusion}

Let's briefly summarize what problems our approach addresses and in which context this takes place. ${ }^{1}$ Psychological and cognitive scientists believe that the human deliberative tasks and our linguistic capacity are symbolic in nature. Especially the proponents of the Good Old Fashioned Artificial Intelligence (GOFAI) use symbolic knowledge representation and reasoning for modelling intelligent behaviour in cognitive agents. However, the symbol system including its mechanisms need to bear meaning in itself. Citing Harnad [1990, p. 340], the symbol grounding problem addresses this issue in terms of "How is symbol meaning to be grounded in something other than just more meaningless symbols?" A grounded meaning of the symbol system may be derived from causal connections with the environment, which allow for a direct interaction without referring to an external observer or programmer.

The symbol grounding problem sets the general context for the more specific symbol anchoring problem. The symbol anchoring problem applies to any cognitive robot in principle, which perceives its environment using sensors, employs actuators in order to interact with its environment, and uses a symbolic representation and reasoning system, where its symbols denote objects of the world. The symbol anchoring problem arises when the sensorimotor and the symbolic representations are completely separate, while different entities of these two distinct representations are intended to refer to the same object of the world (cf. Figure 2.1). Therefore, symbol anchoring aims at creating and maintaining the correct connections between symbols denoting objects and their corresponding perceptual images, both referring to the same physical objects. Our multi-modal approach presented above addresses the symbol anchoring problem and is designed to provide a practical, yet general solution for cognitive, embodied, situated, intelligent robots. Next we will discuss our results.

\subsection{Evaluation}

Symbol anchoring should not be understood as a replacement for object recognition. Our approach and its interface to the object recognition system emphasize the division of responsibilities based on what each system is best suited for. In fact, the symbol anchoring is designed and suited for object identification given the perceptual properties examined by the object recognition and the symbolic knowledge gathered about the individual objects. Conversely, the object recognition system itself is typically not suited for identifying individual objects of the robot's environment, especially in case the objects can be hardly distinguished based on their phenomenal characteristics only, such that high-level reasoning about knowledge and context becomes vital for solving the robot's tasks.

In this sense, our symbol anchoring approach should be considered an abstraction layer in the deliberative level, which performs symbol anchoring independent of the task and application

\footnotetext{
"Our approach" refers to our more general, multi-modal symbol anchoring approach in the sequel.
} 
domain automatically. It interfaces with the perceptual information about objects of the environment provided by the recognition and tracking system on the one side, and the symbolic knowledge about objects on the other side. As a result of each update, our symbol anchoring approach provides the whole set of possible hypotheses of all correspondences between all given percepts and object symbols that are consistent with all the information available so far. Thus our approach alleviates the burden of manually computing which object symbols a given percept could possibly correspond with significantly.

Our approach is based on the basic cognitive motto about beliefs saying that "seeing is normally believing" [Bell and Huang, 1999, p. 1]. In particular, so-called perceptual seemings-hallucination of percepts, in other words - are excluded. This would involve belief revision otherwise, which is not addressed here. Being a typical design in cognitive robotics, the appropriate control architecture for our approach complies with the sense-data theory where non-physical entities form the robot's immediate experience and represent the physical object of the world.

The Fluent Calculus provides the underlying predicate logic language for our approach. Various kinds of references to objects known in the philosophy of language are made available in the predicate logic language in general and in our symbol anchoring approach in particular: indexicals, categories of objects, definite and indefinite references to objects, as well as symbolic descriptions of objects.

\subsubsection{Evaluation with respect to the Symbol Anchoring Problem}

Coradeschi and Saffiotti [2002] specified a list of criteria for evaluating an approach to the symbol anchoring problem, which we summarized in Section 2.3.4. Next, we will discuss our approach with respect to these criteria.

- The requirements include that any approach should allow to refer to an object of the environment by means of a symbolic description. Our approach features symbolic descriptions in terms of definite and indefinite references to objects, for instance. Basically, any well-formed first-order logical formula may serve as a reference to an object.

- Obviously, any symbol anchoring approach ought to be able to determine and to install a new correspondence between a percept and an object symbol appropriately. Our functionality "Find" implements this task and verifies that the symbolic knowledge matches the perceptual information about the object under consideration.

- Once, a correspondence was established, it has to be updated upon arrival of new perceptual information. Our functionality "Track" implements this task, maintaining the anchor representation of the given correspondence. Of course, it ensures that the consistency of the correspondence persists, too.

- However, tracking of an object may fail due to various reasons. Then, upon new percepts of the lost correspondence, a symbol anchoring approach shall be able to identify and to re-establish the correct correspondence. The just described tasks are also realized in our functionality "Find".

- An approach to the symbol anchoring problem shall be able to deal with ambiguous situations and to resolve them. Our approach anchors all available percepts in parallel and maintains all potential hypotheses of correspondences in order to avoid ambiguous situations in advance. Mutual exclusivity constraints among different correspondences contribute to avoiding ambiguity. In the event where an inconsistency among the symbolic and perceptual information associated to an object occurs, this object's correspondence becomes extinct automatically. All available symbolic and perceptual information is utilized during the computation of admissible correspondences and hypotheses of correspondences. Furthermore, we developed the fundamental means for ordering the hypotheses according to their applicability and proposed some domain-independent preference criteria for this purpose. On top of that, a so-called belief state designates the most preferred hypothesis automatically and 
dynamically. However, domain-dependent preference criteria are necessary in order to fully resolve ambiguous situations of symbol anchoring, which was out of scope of our general approach that is designed to be independent of the application domain.

In summary, we believe that our approach satisfies the above, important requirements to the greatest possible extent, and thus represents an effective solution to the symbol anchoring problem.

\subsubsection{Evaluation with respect to the Symbol Grounding Problem}

Several properties are considered crucial for solving the symbol grounding problem and were mentioned in Section 2.2.4. Although symbol grounding poses a more general, abstract and philosophical problem than symbol anchoring, we still want to verify those crucial properties with respect to our approach in the following. The required embodiment of the agent certainly applies to a cognitive robot equipped with sensor devices and actuators for interacting with the real world, which is the typical kind of robot platform for applying our symbol anchoring approach to. Recall that an agent who utilizes environmental context for adapting its cognition, reasoning and acting is called to bear so-called situatedness. Due to the high expressiveness of the predicate logic language underlying our approach and the direct, yet open integration with the knowledge representation and reasoning system, our approach clearly facilitates situatedness. However, the requirement of learning the representation responsible for symbol grounding has not been addressed here.

According to Harnad [1993], the Total Turing Test provides a suitable testbed for the grounding of a symbol system (cf. Section 2.2.4.1). We have shown above that the correct hypothesis - the one which is consistent with the available symbolic and perceptual information-is always among those hypotheses computed by our approach (cf. Section 4.2.6). The correct hypothesis especially guarantees that the "symbols and the symbolic activity cohere systematically with the objects, events and states of affairs that its symbols are interpretable about" regarding object symbols [Harnad, 1993]. This alignment of symbolic and perceptual information in form of epistemic constraints sustains our belief that our symbol anchoring approach fulfils the requirements of the Total Turing Test with respect to grounding of object symbols. Together, the symbol anchoring, the object recognition and the sensorimotor systems working in concert certainly achieve the robotic capacity required further for passing the Total Turing Test: to be able to discriminate, to recognize, to identify, to manipulate and to describe the objects of the robot's environment. In particular our symbol anchoring approach affords the capacity to discriminate, to identify and to describe objects.

In Section 2.2.4.2 we mentioned Harnad's test for evaluating symbol systems with respect to appropriate groundedness of symbols [Harnad, 1990]. The test comprises eight formal criteria in its first part. Referring to these criteria, our symbol anchoring approach and its underlying predicate logic language, the Fluent Calculus, together comply with these criteria because:

1. This symbol system defines certain predicates, functions, constants and macros as the basic symbolic tokens,

2. that are used to define the axioms and rules of this symbol system for manipulating the symbolic representation.

3. The symbolic representation is a well-defined set of symbolic tokens with a certain structure.

4. The definitions of the symbol system in use are syntactic in nature, as the specific axiomatization and domains of discourse depend on the application domain and are thus left aside. All definitions of our approach are designed in a domain-independent way.

5. Both our symbol anchoring approach and the underlying Fluent Calculus define the axioms explicitly how the representation may be rulefully manipulated in general.

6. Fluents could be considered primitive atomic symbol tokens, 
7. while they constitute states and logical formulas about knowledge - which in turn represent composite symbol tokens.

8. Our symbol anchoring axioms and the Fluent Calculus are based on the usual semantics of first order logic. Moreover, the axiomatization of the application domain of a cognitive robot also includes the domains of discourse, e.g., the kinds of objects, individual objects, perceptual attributes and their perceptual values. Altogether, they constitute the semantics of the intended application.

The second part of Harnad's test for grounded symbol meaning requires a symbol system to be able to discriminate, to identify and to describe the objects and states of affairs to which its symbols refer (see Section 2.2.4.2). As we argued above, these tasks count among the major objectives of our symbol anchoring approach. In particular, different objects can be told apart, individual objects can be identified as well as described using various means. Hence, we believe that our approach to the symbol anchoring problem is able to pass both parts of Harnad's test for grounded symbol meaning.

Our approach uses a general interface to linguistic terms denoting phenomenal properties of objects. We have implemented a basic solution to the symbol grounding problem with respect to these linguistic terms - the domain axiomatization includes static relations mapping the linguistic terms to intervals of perceptual values. This way, vagueness of linguistic terms is taken into account, but the grounding of the symbols is static and pre-defined, which is required by our approach in order to ensure the consistency of symbol anchoring and reasoning over time. It seems that incorporating methods of machine learning and belief revision could remedy this deficiency, but this was out of scope of our project and would necessitate complex modifications.

Next, we want to evaluate our symbol anchoring approach with respect to the so-called Zero Semantical Commitment Condition (Z-Condition for short), defined by Taddeo and Floridi [2005] and summarized in Section 2.2.4.3. According to the strict requirements of the Z-Condition, the cognitive agent has to elaborate its symbolic representation and semantics from scratch. Let's discuss this condition with respect to each individual kind of symbol used in the representation of our approach.

- The domain axiomatization may include a priori known object identifiers and symbolic descriptions thereof. Using a priori known object identifiers and their specifications would violate the Z-Condition, but their use is not mandatory in our approach.

- Instead, our approach allows the cognitive robot to completely experience the individual objects of the environment itself and to introduce object identifiers upon experiencing new objects dynamically. The cognitive robot can learn symbolic information about these objects from communication and interaction with other individuals in the environment for instance, or derive information from what is known so far. Hence, the Z-Condition shall be preserved.

- All percept identifiers are obtained from the object recognition system dynamically during run-time of the symbol anchoring system. According to the phenomenalist's view of cognition, the percept identifiers derive their semantics from the real world, which complies with the Z-Condition.

- Symbolic descriptions of objects are purely based on or derived from symbolic information, and thus do not violate the Z-Condition themselves.

- As we described above, the grounding of linguistic terms denoting perceptual attributes is simply pre-defined in our approach. Obviously, this violates the Z-Condition. A machine learning approach could possibly abolish this semantic bias, but implementing it was out of scope of our project.

In general, the structure of the symbolic representation of our approach is pre-defined, but a cognitive robot elaborates the contents itself. Accordingly, our symbol anchoring approach partly violates the Z-Condition due to pre-defined symbolic representations. However, it was not our 
primary objective to completely solve the much stricter and more abstract problem of symbol grounding, too.

Furthermore, Taddeo and Floridi [2005] proposed what a valid solution to the symbol grounding problem requires of any symbol system, which we summarized in Section 2.2.4.3. Evaluating our approach to the symbol anchoring problem with respect to these requirements provides us the following insights into the groundedness and the focus of our approach in comparison with the much more general scope of symbol grounding.

1. Object recognition in combination with our symbol anchoring approach can be considered a bottom-up process for grounding percepts to object symbols as required. For each percept representing a physical object our symbol anchoring axioms determine appropriate correspondences with object symbols in a bottom-up manner.

2. Conversely, symbolic information about objects also influences the symbol anchoring in a top-down feedback process as required. All the symbolic information is taken into account automatically in determining the appropriate correspondences between object symbols and percepts, and has to comply with all sensorimotor perception and interaction.

3. Our symbol anchoring approach allows the cognitive robot to develop its symbolic representation of objects dynamically on its own, although not the complete representation as required by Taddeo and Floridi [2005]. However, the representation of symbolic and perceptual information about objects can be extended and modified.

4. While our symbol anchoring approach makes use of categories of objects, it is not designed to actively categorize percepts.

5. We did not address the capacity of the cognitive robot to communicate and to synchronize its own symbol anchoring representation with those of other robots as Taddeo and Floridi [2005] required.

6. An evolutionary approach in the development of the above functionalities was not the focus of our work. The definitions and axioms of our approach have been designed by the authors, which infringes the requirement of Taddeo and Floridi [2005] in principle.

7. As several important concepts have been incorporated in the design of our symbol anchoring approach, which influence the computation fundamentally, this semantic, external bias breaks the Z-Condition required for the development of the above items (1) to (6). It is yet unclear to us, how this requirement could ever be realized in a practical system for symbol anchoring.

In summary, we believe that our approach contributes to some significant extent to solving the symbol grounding problem. As the frame of reference of the cognitive robot in symbol anchoring is the real world, percepts of physical objects represent the basis of meaning of object symbols in symbol grounding. Thus, we believe that real-world perception forms a legitimate answer to Harnad's question "How is symbol meaning to be grounded in something other than just more meaningless symbols?" in terms of cognitive robotics [Harnad, 1990, p. 340].

\subsubsection{Summary of Contribution}

Next, we briefly highlight the fundamental characteristics of our approach to the symbol anchoring problem, all of which contribute to its expressiveness, completeness and flexibility.

- Our approach determines all possible correspondences with object symbols for all available percepts at once. This method minimizes ambiguity in symbol anchoring.

- Depending on the given configuration of correspondences of the considered percept and object symbols, the appropriate symbol anchoring functionality is chosen and applied automatically. This way, our approach adapts the symbol anchoring to the particular configuration of each individual correspondence. 
- All kinds of phenomenal and functional characteristics, features of interaction and relations with other objects of the environment are used in determining the possible correspondences and to constrain the potential hypotheses of correspondences. Especially any new information may also invalidate an existing hypothesis in case of inconsistencies. Both extending and shrinking of the hypothesis space are performed automatically.

- The community identified that bidirectionality of the links, relations and processes of symbol anchoring between the perceptual and symbolic levels is beneficial. For instance, the consistency predicate of our approach implements consistency constraints in both directions: bottom-up and top-down.

- As the cognitive robot interacts with and acts in the real world, it has to be able to deal with ubiquitous uncertainty in perception. Our approach explicitly represents and reasons about perceptual uncertainty. All percepts referring to the same physical object are fused into a unique perceptual signature of the object, which minimizes the perceptual uncertainty over time.

- We have implemented a symbol anchoring approach that allows to deal with incomplete knowledge about objects easily. As no object needs to be known initially, all objects may be experienced later during runtime. A symbolic description of a certain class of objects or of an individual object may be specified incompletely, yet our approach allows to reason about all objects and to anchor the objects to percepts.

- Linguistic propositions of objects typically denote phenomenal characteristics on the symbolic level. In our approach such symbols derive their meaning from grounding in ranges of real-world, perceptual values, thus taking vagueness into account explicitly.

- Various kinds of references to objects are employed in the philosophy of language. Due to high expressiveness of the underlying logical language, our symbol anchoring approach provides a multitude of kinds of references including definite references, indefinite references and proper names. In principle, any well-formed first-order logical formula may constitute a valid reference to an object.

Our symbol anchoring approach is based on several fundamental concepts that we consider crucial for anchoring symbols successfully and effectively. In the following we briefly summarize these concepts, which have been discussed in the previous chapters in detail.

- Although various, available symbolic and perceptual information may provide good evidence for a given percept to correspond to a certain object identifier, there is simply no guarantee that this correspondence is actually correct. Therefore, the symbol anchoring system must be able to revoke a correspondence once it was recognized to be wrong and to precisely revoke any information and representation involved. For this purpose, our symbol anchoring approach separates information derived from the symbolic level from information coming from the perceptual level clearly. This concept facilitates to retract an invalid hypothesis and to avoid an irreversibly intermingled representation.

- Organizing objects in multiple, different categories helps to focus the symbol anchoring and object recognition on the relevant kind of objects in our approach. This facilitates to optimize the performance of anchoring, because our approach anchors all available percepts in parallel. It also limits the number of potential candidates of a correspondence, hence reduces ambiguity regarding irrelevant, alternative hypotheses. Our approach benefits from using categories of objects to introduce context dependency to the meaning of linguistic propositions.

- Every single percept potentially represents a candidate for anchoring a given object symbol to it. Thus, no percept should be neglected in symbol anchoring. For this purpose, our symbol anchoring approach associates each percept with some object identifier. We have proved that our approach complies with this concept. 
- All symbolic and perceptual knowledge about all objects of the environment, that complies with a certain interpretation of the situation, forms a hypothesis of correspondences. Our approach determines and maintains all potential hypotheses of correspondences in parallel in order to facilitate reasoning about alternative interpretations for resolving ambiguous anchoring situations. This concept also allows to switch to an alternative hypothesis easily, if the allegedly correct one was identified to be invalid. In this case, the invalid hypothesis can simply die out, as we have shown that the correct hypothesis must be among the remaining hypotheses.

- There exist certain natural, logical constraints among different hypotheses of correspondences. We have proved our symbol anchoring approach to only generate such hypotheses that are consistent with those constraints.

In summary, thanks to the above-mentioned characteristics and fundamental concepts, we believe that our approach has gained yet unmatched expressiveness, generality and precision and implements the full functionality typically required of symbol anchoring approaches.

\subsection{Future Work}

Certainly, our approach bears some limitations and shortcomings, too, which we will discuss in this section.

- The symbol anchoring methodology of our approach disregards the anchoring of such object symbols which denote composite objects and of such percepts that represent only a part of a composite, physical object on purpose. Otherwise, this would mean to require the capacity to anchor composite objects, which in turn would call for a number of additional, complex problems to be solved, that are out of scope of our approach, e.g. spatial reasoning. The basic principles behind composite objects, e.g. structure and dynamics of the individual parts, seem to depend on the application domain critically, which does not fit to our general approach to the symbol anchoring problem.

- So far, we have developed the theory of our approach based on the logic language called the Fluent Calculus. It would be interesting to see how an implementation of our approach performs with a real cognitive robot. The Fluent Calculus has been implemented in the logic programming language FLUX, which is the ideal framework for a practical symbol anchoring system based on our theory [Thielscher, 2005]. Unfortunately, implementing our approach was out of scope of the project due to time constraints. We expect that the experience gained from implementing and testing the symbol anchoring approach in a real-world system will certainly return valuable feedback, e.g. for refining the interface to the object recognition system. We already pointed out the value of our symbol anchoring approach above; needless to say that a real-world symbol anchoring system based on our approach would advance standard cognitive robotics architectures significantly.

- The management of symbolic properties of objects could be improved towards a more modular fashion. While our approach simply associates certain properties with a certain category of objects, an object's category could also serve to inherit common properties from more general categories within a hierarchical structure of object categories. Possibly, a certain object may need to belong to multiple categories of objects. Ontologies - as typically used in Description Logics - seem to offer an interesting avenue of extending our approach.

- Our symbol anchoring approach presupposes that the object recognition system categorizes percepts. Today's robust and successful object recognition systems seem to be suitable for this task. Moreover, our approach assumes that no misclassification occurs, because belief revision is not taken into account. However, it may be an interesting improvement on our approach to be able to deal with such misclassifications using abductive reasoning. 
Jin and Thielscher [2007] already provided a comprehensive guide on belief revision based on the Fluent Calculus. It is certainly worthwhile to check how their approach to belief revision could be integrated with our approach to symbol anchoring. This way, it could become possible to not only neglect a symbol anchoring hypothesis, but to revise parts of a hypothesis.

- We have implemented a rather simple approach to grounding symbols denoting phenomenal characteristics as they are statically associated with ranges of perceptual values. An appropriate solution to the symbol grounding problem would require the cognitive robot to autonomously learn the grounding and to adapt it dynamically and continuously. As this would interfere with how our approach maintains and reasons about the hypotheses of correspondences, the aspect of learning to ground symbols was out of scope of our project. Again, it seems to call for belief revision.

- Our approach as presented above is limited to handle objects of categories which are initially known. In practice with an object recognition system in place which is capable of learning and classifying new, additional categories of objects, the symbol anchoring system also needs to be able to extend the set of known kinds of objects on the symbolic level. In cases where previously known objects retain their object category, this extension to our approach seems to be rather straightforward. Otherwise, learning and revising the category of an object would involve belief revision, which was beyond the scope of our approach.

- Recall the major objectives of symbol anchoring - to determine and to maintain the correct correspondences between symbols denoting objects of the environment and their perceptual images. Our symbol anchoring approach computes all possible hypotheses of correspondences and can determine the most preferred hypothesis, which includes the most likely correspondence for each object, using a belief state and a suitable preference criterion. However, the preference selection is not necessarily correct; thus the belief state can by no means guarantee that the correspondences included in the most preferred hypotheses are actually correct. Therefore, the planning system of the cognitive robot should analyse the hypothesis space right after the anchoring system has determined all the hypotheses of correspondences. To name just a few examples, the following items represent relevant and helpful features in analysing the hypothesis space, extending our symbol anchoring approach towards a robust, integrated anchoring and planning system:

- Do all anchors of the object identifier at hand point to the same percept in all available hypotheses, or vice versa?

- How many belief states exist in the given situation, and do all belief states only include such anchors that refer to the same percept, or vice versa?

- Depending on the kind of reference requested, the certainty of it referring to the correct physical object does matter or not. In contrast to the definite reference, any candidate object of an indefinite reference that exhibits all required characteristics of the requested class of objects serves the purpose (cf. e.g. Bouguerra et al. [2006], Karlsson et al. [2008]).

- How distinctive is the object of interest in terms of the available symbolic and perceptual information with respect to other objects in the given situation?

In addition, domain specific heuristics seem to offer useful hints for interpreting the situation with respect to the degree of support of the current belief state. Depending on the outcome of the analysis, the planning system of a cognitive robot may need to invoke an appropriate anchoring or sensing strategy before interacting with the assumed physical object if the evidence for the correctness of the correspondence of interest is too low. For example, while it makes perfect sense in certain application domains and situations to stick to the current belief state and to try acting on the physical object assumed to correspond to the 
object symbol in question, the same strategy would pose too high a risk in mission critical applications due to an unacceptably low level of confidence in the given correspondence. However, the above ideas - extending our symbol anchoring approach towards planningdisagree with our major objectives. Such extensions tend to take effect only if tailored to the intended application domain, but our approach was designed for general applicability providing all basic functionalities of symbol anchoring commonly required by typical cognitive robots interacting with a real environment. Furthermore, such extensions tend to intermingle reasoning, planning and execution of actions, which would impair the modularity of the robot control architecture, thus limiting its flexibility and interoperability. Therefore, we disregarded to incorporate planning and execution of actions (especially of sensing actions) in our approach. Nevertheless, we take the opportunity to discuss important directions of extending our approach in terms of planning below.

- Recall that the practical benefit of symbol anchoring in general and of our approach in particular for a cognitive robot is to ensure that the symbolic knowledge representation is in sync with the real world in terms of references to objects. Before a cognitive robot interacts with the object of interest, the robot's planning system should make sure that the correspondence of this object is grounded on an appropriate real-world percept. It is the symbol anchoring system's task to determine an appropriate percept and to establish a grounded correspondence if possible, such as our approach does. However, the planning system is responsible for verifying the groundedness of the correspondence right before acting on the object. Therefore, we suggest that the planning system should pose the precondition that the correspondences of all those objects, which the robot is about to act on next, have to be grounded. Obviously, the symbol anchoring system critically depends on the latest perceptual information about the kind of objects of interest. For this purpose, the planning system should plan to execute an appropriate sensing action just before the symbol anchoring step. Based on the tools for computing the hypothesis space of correspondences provided by our symbol anchoring approach, a planning system should verify the groundedness of the requested references to objects. Building such a planning system of a cognitive robot was out of scope of our project in terms of our focus of research.

- It's worth mentioning a few thoughts on what constitutes an appropriate planning strategy of a cognitive robot which integrates with symbol anchoring nicely. Given the requested reference to a certain object of interest, the primary objective of the planning system should be to make sure that a suitable correspondence is available and grounded (cf. the previous item). In order for an object to fit the given reference, all of its properties have to be verified in general. Depending on the kind of reference requested, either the symbolic description of the reference has to be matched against the properties of objects experienced so far in all detail, or the reasoning system deduces an individual, already known object. In the end, the symbol anchoring system verifies whether the correspondences determined this way are actually based on appropriate percepts. Anyway, the symbol anchoring system can only examine the perceptual information that is available, because it does neither plan nor execute sensing actions by itself (cf. previous items). Thus, the planning system should include appropriate sensing actions that provide the phenomenal attributes of the objects of interest which are to be matched against the specification of the reference. For example, after the object recognition system found objects of the given category in the robot's environment during exploration, more specific sensing actions could examine individual physical objects in detail one after another. Our symbol anchoring approach will keep the space of hypotheses of correspondences up-to-date and in sync with the real-world percepts automatically.

Let's briefly discuss an exemplary planning strategy of a mobile, cognitive robot servicing in an office or household environment. Given the task to physically act on an individual object specified in terms of a definite reference, the planning system evaluates the knowledge about this object. In case its position is known, the robot should first navigate there and search for the object in the immediate vicinity of the object's assumed position. Otherwise, if the object's position cannot be inferred at all from the current information, the reference 
is considered in the same way as an indefinite reference such that the available information about the object describes what constitutes a suitable object that is to be looked for in the environment. Depending on the specificity of the object's description, no, few or many objects perceived in the environment may match. A cautious planning strategy examines one candidate object after another at the highest perceptual resolution available in order to distinguish individual objects and to gather sufficient phenomenal details. Dynamic properties of objects also help to prefer the correct percept over other similar candidates. Reasoning about previous interactions with objects and about their properties perhaps yields the most valuable means for object identification. To this end our symbol anchoring approach facilitates a tight integration with the reasoning system.

- So-called object affordances seem to offer a very useful extension of our symbol anchoring approach. Explicit, functional knowledge about the possible ways of interacting with certain kinds of objects could be directly utilized for identifying individual objects. Being part of an object model, such affordances of objects can be applied during observing how objects are used in the environment by other agents, humans or the robot itself. Moreover, the planning system could employ specific actions just for the purpose of verifying the identity of a perceived object in terms of its functional properties. Characteristics learned from interacting with an object in certain ways yield valuable information about its affordances. For example, imagine the robot's task is to pick up a certain bottle of wine from a table, where the particular bottle of interest is known to be full and to bear an opaque exterior. Thus, object recognition is not sufficient in order to verify the bottle's property of being full. If the robot could measure the weight of the bottle using its own sensing device or using some weighing scales nearby, verifying this property sustains the object identification substantially. In general, reasoning about and planning with object affordances certainly supplements the effectiveness of symbol anchoring.

- According to phenomenalism known in cognitive philosophy, relations between objects are considered important characteristics of objects with respect to object identification and communication. Recognizing relations between real-world objects certainly presupposes a good performance of the object recognition system as it is a difficult task. For instance, answering the seemingly straightforward question of whether a certain object $A$ is larger than, smaller than or of equal size as some object $B$ crucially depends on the robot's capability to perceive and to interpret three-dimensional space and objects placed therein in arbitrary ways correctly, taking into account such effects like obstructions, noise, shape, illumination and shadow, the robot's perspective, technical parameters of sensing devices, etc. Also, the symbolic level requires at least a fundamental understanding of the concept of space and needs to be able to reason about space in general. Note that our symbol anchoring approach inherits its underlying logical formalism's basic ability to represent and to reason about relations between objects, which may be employed directly for specifying symbolic descriptions of objects. Nevertheless, integrating our symbol anchoring approach with a state-of-the-art object recognition and spatial reasoning component would quite certainly bring an asset for a cognitive robot. 


\section{Appendix A}

\section{List of Symbols Used}

This section describes all symbols concisely in alphabetical order, which our uni-modal and multimodal approaches introduced.

\section{A.1 Sorts}

ATTR: The sort ATTR comprises all perceptual attributes that the cognitive robot knows of in a certain application domain. They are used by our approach for reasoning about both symbolic and perceptual information. This sort also includes all those attributes that the object recognition system may report about objects of the environment (cf. Section 3.5 on page $29 \mathrm{ff}$.).

OBJCAT : Our approach distinguishes between different kinds of objects that typically have quite different characteristics. The sort OBJCAT includes all those categories of objects known by the cognitive robot in the intended application domain and all those which are distinguished by the object recognition system (cf. Section 3.3 on page $27 \mathrm{ff}$.).

PERCID : The countably infinite sort PERCID holds identifiers of percepts. Our approach simply uses natural numbers, i.e. $\operatorname{PERCID}=\mathbb{N}$. Each percept reported by the object recognition system bears an identifier of this sort (cf. Section 3.4 on page $28 \mathrm{ff}$.).

PERCPRED : All perceptual predicates that the cognitive robot knows of are contained in the sort PERCPRED. Perceptual predicates are used to denote attribute values of phenomenal characteristics in our approach (cf. Section 3.6 on page $31 \mathrm{ff}$.).

PERCSIG : The perceptual signature is defined as an aggregate structure of sorts in our approach:

$$
\text { PERCSIG } \stackrel{\text { def }}{=} \mathcal{P}((\text { ATTR } \times(\mathbb{R} \times \mathbb{R}))
$$

Accordingly, a perceptual signature $\Gamma$ : PERCSIG is a set of pairs, where each pair consists of a perceptual attribute and a tuple of perceptual values (cf. Section 4.1.1 on page $43 \mathrm{ff}$.).

$\mathcal{X}$ : $\quad$ The sort $\mathcal{X}$ includes all symbols of objects that the cognitive agent knows initially. Both $\mathcal{X}$ and PERCID constitute the set of all object identifiers that objects may be associated to in our approach (cf. Section 3.4 on page $28 \mathrm{ff}$.).

\section{A.2 Fluents}

$A / 4$ : The uni-modal fluent $A / 4$ represents an "anchor" with the signature

$$
A: \mathcal{X} \cup \text { PERCID } \times \text { OBJCAT } \times \text { PERCSIG } \times \text { PERCID } \cup\{\perp\} \mapsto \text { FLUENT }
$$


An instance $A(x, o, \Gamma, \pi)$ represents the correspondence between an object identifier $x$ of category $o$ and a percept denoted by identifier $\pi$. The so-called best perceptual estimate $\Gamma$ includes all perceptual information that has been gathered from the percept $\pi$ and all previously corresponding percepts (cf. Section 4.1.2 on page $45 \mathrm{ff}$.).

$A / 5$ : The multi-modal "anchor" fluent $A / 5$ only differs to the above fluent $A / 4$ in its additional fourth component. Our multi-modal symbol anchoring approach defines this fluent as:

$$
A: \mathcal{X} \cup \text { PERCID } \times \text { OBJCAT } \times \text { PERCSIG } \times \mathcal{P}(\text { PERCID }) \mapsto \text { FLUENT }
$$

The fourth component comprises all currently and previously corresponding percept identifiers in our multi-modal approach (cf. Section 5.3.2 on page $83 \mathrm{ff}$.).

$C / 4$ : The fluent $C$ is used in the multi-modal symbol anchoring axiom as an auxiliary fluent to temporarily store the correspondence determined by the symbol anchoring functionalities. Its signature is:

$$
C: \text { PERCID } \cup \mathcal{X} \times \mathcal{P}(\text { PERCID }) \times \text { FLUENT } \times \text { FLUENT } \mapsto \text { FLUENT }
$$

An instance $C\left(x, \Pi_{P}, f^{-}, f^{+}\right)$represents a certain, chosen correspondence between object symbol $x$ and block $\Pi_{P}$ of associated percepts. The fluents $f^{-}$and $f^{+}$hold the outdated and new anchor fluents respectively (cf. Section 5.4 .3 on page $88 \mathrm{ff}$.).

Offset/1: An instance Offset $(n)$ of this fluent holds the number $n$ which represents the minimal natural number to be used as a fresh object identifier denoting a new object in our uni-modal approach. This way, we make sure that all object identifiers are unique. We define fluent Offset as follows (cf. Section 4.2 .4 on page $58 \mathrm{ff}$.).

$$
\text { Offset : PERCID } \mapsto \text { FLUENT }
$$

Prop/3: The fluent Prop serves as a general means to represent symbolic knowledge about phenomenal characteristics of an object, which our approach defines as follows.

$$
\text { Prop }: \mathcal{X} \cup \text { PERCID } \times \text { ATTR } \times \text { PERCPRED } \mapsto \text { FLUENT }
$$

An instance $\operatorname{Prop}(x, a, p)$ tells the agent that the object $x$ has the property $p$ of the perceptual attribute $a$ (cf. Section 3.7 on page $33 \mathrm{ff}$.).

\section{A.3 Functions}

NoP/1: The function $N o P(s)$ returns the number of available percepts in a given situation $s$ and is defined as follows (cf. Section 4.1 .1 on page $43 \mathrm{ff}$.).

$$
\text { NoP : SIT } \mapsto \mathbb{N}
$$

\section{A.4 Predicates}

AnchorUpdate/7: The multi-modal predicate AnchorUpdate $\left(o, \Gamma_{A}, \Pi_{A}, \Pi_{P}, \Gamma^{\prime}, \Pi^{\prime}, s\right)$ combines the given set $\Pi_{P}$ of current, associated percepts of the situation $s$ with the set $\Pi_{A}$ of percepts already integrated into the anchor fluent, yielding the new set $\Pi^{\prime}$ of percepts. Moreover, this predicate fuses the current best, perceptual estimate $\Gamma_{A}$ stored in the anchor with all the new, perceptual information provided by the percepts of set $\Pi_{P}$ into the successive perceptual estimate $\Gamma^{\prime}$ (cf. Section 5.4 .4 on page $91 \mathrm{ff}$.).

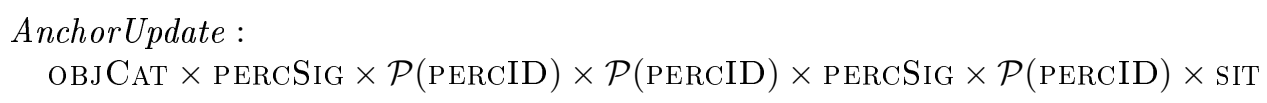


AttrTypeStatic/2: The predicate AttrTypeStatic(o,a) holds iff the given attribute $a$ is considered static regarding the category of objects in the application domain at hand (cf. Section 3.6 on page $31 \mathrm{ff}$.).

\section{AttrTypeStatic : OBJCAT $\times$ ATTR}

BState/2: Given an appropriate preference criterion, the predicate BState $(s, z)$ designates one or more most preferred hypotheses $z$ of correspondences in the given situation $s$ (cf. Section 4.3 .1 on page $68 \mathrm{ff}$.).

\section{BState $:$ SIT $\times$ STATE}

BStateSet/2: The predicate BStateSet $(s, \zeta)$ holds for a certain set $\zeta$ of hypotheses of the given situation $s$. The preference criterion in use determines all hypotheses of the set $\zeta$ to be the most preferred ones (cf. Section 4.3 .2 on page $69 \mathrm{ff}$.). Thus, the predicate BStateSet is defined less rigid compared to predicate BState.

$$
\text { BStateSet : } \operatorname{SIT} \times \mathcal{P}(\operatorname{STATE})
$$

$C C / 8$ : The predicate $C C\left(\pi_{P}, o, \Gamma_{P}, n, f^{-}, f^{+}, s, z\right)$ combines all three symbol anchoring functionalities, which determine all possible correspondences of the given percept $\pi_{P}$. Among those possible correspondences, it chooses a particular one non-deterministically (cf. Section 4.2.1 on page $51 \mathrm{ff}$.). $C C$ is short for "choice of correspondences".

$$
C C: \text { PERCID } \times \text { OBJCAT } \times \text { PERCSIG } \times \text { PERCID } \times \text { FLUENT } \times \text { FLUENT } \times \text { SIT } \times \text { STATE }
$$

Consistent/1: The predicate Consistent $(z)$ holds true for the given hypothesis representation $z$ iff $z$ complies with certain consistency constraints (cf. Section 4.1 .2 on page $45 \mathrm{ff}$.).

\section{Consistent : STATE}

DiffCorr $/ 2$ : The predicate DiffCorr $\left(z_{1}, z_{2}\right)$ is defined to hold true, iff the given states $z_{1}$ and $z_{2}$ represent different symbol anchoring hypotheses (cf. Section 4.3 .1 on page $68 \mathrm{ff}$.).

$$
\text { DiffCorr : STATE } \times \text { STATE }
$$

Grounded/4: The predicate Grounded $(o, a, p,(b, t))$ serves the purpose of grounding perceptual predicates. More precisely, a perceptual predicate $p$ specifying the perceptual attribute $a$ for objects of category $o$ is grounded on the interval $(b, t)$ of perceptual values (cf. Section 3.6 on page 31 ff.).

$$
\text { Grounded : OBJCAT } \times \text { ATTR } \times \text { PERCPRED } \times(\mathbb{R} \times \mathbb{R})
$$

Intersect/2: The predicate Intersect $((a, b),(c, d))$ holds true iff the given intervals $(a, b)$ and $(c, d)$ of real numbers intersect (cf. Section 4.2 .2 on page $52 \mathrm{ff}$.).

$$
\text { Intersect }:(\mathbb{R} \times \mathbb{R}) \times(\mathbb{R} \times \mathbb{R})
$$

IsGrounded $/ 3$ : The predicate IsGrounded $(x, s, z)$ tells us, whether the given object $x$ is currently grounded in the given situation $s$ and hypothesis $z$ (cf. Section 4.2 .8 on page $65 \mathrm{ff}$.).

$$
\text { Is Grounded }: \mathcal{X} \cup \operatorname{PERCID} \times \operatorname{SIT} \times \text { STATE }
$$


IsNewSymbol/3: The auxiliary predicate IsNewSymbol is part of our uni-modal symbol anchoring approach. Predicate IsNewSymbol $\left(x, s, z^{\prime}\right)$ holds true iff the given object symbol $x$ is a fresh one in the successor hypothesis $z^{\prime}$ after performing the symbol anchoring transaction and update in situation $s$ (cf. Section 4.3.3.1 on page $72 \mathrm{ff}$.).

$$
\text { IsNewSymbol : PERCID } \times \text { SIT } \times \text { STATE }
$$

Match/3: The predicate $\operatorname{Match}(x, \Gamma, z)$ verifies whether the symbolic information known about the object $x$ matches the perceptual information $\Gamma$ obtained from a percept in the given hypothesis $z$. The definition of the uni-modal predicate Match closely resembles the one of the multi-modal version (cf. Section 4.2 .2 on page $52 \mathrm{ff}$. and Section 5.4.4 on page $91 \mathrm{ff}$.).

$$
\text { Match }: \mathcal{X} \cup \text { PERCID } \times \text { PERCSIG } \times \text { STATE }
$$

$P / 4$ : The predicate $P(\pi, o, \Gamma, s)$ represents a current percept of situation $s$ in our uni-modal symbol anchoring approach. This predicate includes the perceptual information $\Gamma$ about a certain object of the environment, which is of category $o$. The identifier $\pi$ denotes this percept (cf. Section 4.1 .1 on page $43 \mathrm{ff}$.).

$$
P: \text { PERCID } \times \text { OBJCAT } \times \text { PERCSIG } \times \text { SIT }
$$

$P / 5$ : $\quad$ Likewise to the uni-modal predicate $P / 4$ representing a percept, the multi-modal predicate $P\left(\pi, o, \Gamma, \pi_{R}, s\right)$ holds the perceptual information about a certain object of the environment. In addition to the uni-modal $P / 4$, the multi-modal version includes the parameter $\pi_{R}$, which may refer to another current percept indicating that both $\pi$ and $\pi_{R}$ are measured of the same physical object (cf. Section 5.3.1 on page $82 \mathrm{ff}$.).

$$
P: \text { PERCID } \times \text { OBJCAT } \times \text { PERCSIG } \times \text { PERCID } \cup\{\perp\} \times \text { SIT }
$$

PBlock/2: The predicate $P B \operatorname{lock}(\Pi, s)$ holds true in our multi-modal approach iff the set $\Pi$ includes all those and only those identifiers of percepts that are associated with each other according to the reference percept of predicate $P / 5$ (cf. Section 5.4 .1 on page $85 \mathrm{ff}$.).

$$
\text { PBlock : } \mathcal{P}(\text { PERCID }) \times \text { SIT }
$$

PFusion/4: The multi-modal predicate PFusion/4 is a particular instance of the multi-modal predicate AnchorUpdate/7. Given a set $\Pi_{P}$ of current, associated percepts of situation $s$, the predicate PFusion $\left(o, \Pi_{P}, \Gamma^{\prime}, s\right)$ fuses the perceptual information provided by the individual percepts into a single perceptual estimate $\Gamma^{\prime}$. All percepts of the set $\Pi_{P}$ of associated percepts originate from the same object of the environment (cf. Section 5.4.6 on page $95 \mathrm{ff}$.).

$$
\text { PFusion : OBJCAT } \times \mathcal{P}(\text { PERCID }) \times \text { PERCSIG } \times \text { SIT }
$$

PKnown/3: Given a certain percept in form of predicate $P\left(\pi_{P}, o, \Gamma, \pi_{R}, s\right)$ in the multi-modal approach, the auxiliary predicate $\operatorname{PKnown}\left(\Pi_{A}, \pi_{P}, \pi_{R}\right)$ checks whether this percept is associated with some object symbol via the symbol's anchor. For this purpose, the anchor fluent includes the set $\Pi_{A}$ comprising all previously corresponding percepts (cf. Section 5.4.4 on page $91 \mathrm{ff}$.).

$$
\text { PKnown : } \mathcal{P}(\text { PERCID }) \times \text { PERCID } \times \text { PERCID } \cup\{\perp\}
$$

Pref $/ 3$ : The predicate Pref $\left(s, z, z_{2}\right)$ implements a user defined, application dependent preference criterion between any two symbol anchoring hypotheses $z$ and $z_{2}$ of a situation $s$ (cf. Section 4.3 .1 on page $68 \mathrm{ff}$.).

$$
\text { Pref }: \text { SIT } \times \text { STATE } \times \text { STATE }
$$


$\operatorname{PrefPercKS} / 4$ : The auxiliary predicate $\operatorname{PrefPercKS}\left(s, z_{1}, z_{2}, \pi\right)$ may serve as a building block for implementing a preference criterion for symbol anchoring hypotheses. We defined this predicate to prefer a hypothesis $z_{1}$ of situation $s$ over another hypothesis $z_{2}$ iff percept $\pi$ corresponds to some already known object identifier in $z_{1}$, and to some fresh object identifier in $z_{2}$ (cf. Section 4.3.3.1 on page $72 \mathrm{ff}$.).

$$
\text { PrefPercKS }: \text { SIT } \times \text { STATE } \times \text { STATE } \times \text { PERCID }
$$

PrefSets $/ 3$ : Based on sets of symbol anchoring hypotheses of a given situation $s$, the preference predicate PrefSets $\left(s, \zeta_{1}, \zeta_{2}\right)$ defines all hypotheses of set $\zeta_{1}$ to be preferred over all those of set $\zeta_{2}$ according to a user defined, application dependent preference criterion (cf. Section 4.3 .2 on page 69 ff.).

$$
\text { PrefSets }: \operatorname{SiT} \times \mathcal{P}(\text { STATE }) \times \mathcal{P}(\text { STATE })
$$

UpdatePS/4: The uni-modal predicate UpdatePS is employed in our approach for the purpose of updating the best perceptual estimate stored in the anchor of a certain correspondence upon obtaining some new perceptual information. More precisely, given the best perceptual estimate $\Gamma_{A}$ of the anchor fluent and the new perceptual information $\Gamma_{P}$ of the percept, UpdatePS $\left(o, \Gamma_{A}, \Gamma_{P}, \Gamma^{\prime}\right)$ fuses both yielding the new, best perceptual estimate $\Gamma^{\prime}$ to be stored in the successive anchor fluent (cf. Section 4.2.3 on page $55 \mathrm{ff}$.).

$$
\text { UpdatePS : OBJCAT } \times \text { PERCSIG } \times \text { PERCSIG } \times \text { PERCSIG }
$$




\section{Appendix B}

\section{Notation}

The wording, spelling and grammar of this work was chosen in accordance with current British English as described in [Hornby, 2000]. The technical writing shall comply with the standards defined in [The University of Chicago, 1982].

- For the sake of ease of understanding and readability, we chose a very similar notation and terminology as used in the approaches by Saffiotti et al. described in Section 2.3.5. We also hope to improve the understanding of the included formulas by specifying the signature of logical macros, although this is not necessary.

- Our approach is based on first-order logic with sorts and arithmetic operators including equality. Using sets in our formalization improves the readability, while sets could directly be replaced with fluents, states and the macro Holds.

- We utilized the symbol " $\equiv "$ denoting the logical equivalence and the symbol " $\supset$ " denoting the logical implication in our work.

- We used the expression $(\exists ! x) P(x)$, the so-called uniqueness quantification, which abbreviates that there is exactly one instance of $x$ such that $P(x)$. The following equivalence holds:

$$
[(\exists ! x) P(x)] \equiv[(\exists x)(P(x) \wedge(\forall y)(P(y) \supset(x=y)))]
$$

- In accordance with common first-order logic notation, all free variables of formulas included in this work are implicitly bound to the universal quantifier " $\forall$ " if not specified explicitly.

- Typically, we used capital letters to denote constants and small letters to denote variables. Greek letters have been used for variables only, due to the above compliance with Saffiotti's formulas. In particular, capital Greek letters stand for sets, but small Greek letters typically represent elements of sets in our work. Variables that carry an apostrophe, e.g. $x^{\prime}$, typically represent the update of the considered variable $x$, e.g. in the successor situation. We typically use the subscript " ${ }_{P}$ " with variables indicating that this value originates from a percept, while the subscript " $A$ " indicates that the origin is an anchor.

- The symbol " $\perp$ " was used as a logical constant in terms, not as a formula. In our work it stands for an ungrounded correspondence. Again, the choice of this symbol was for reasons of compliance with the notation of approaches by Saffiotti et al. as described in Section 2.3.5. 


\section{Bibliography}

C. E. Alchourrón. On the logic of theory change: contraction functions and their associated revision functions. Theoria, 48:14-37, 1982.

P. Althaus and H. I. Christensen. A framework for anchoring in hybrid deliberative systems. Submitted to Autonomous Robots, 2003.

M. L. Anderson. Embodied cognition: A field guide. Artificial Intelligence, 149(1):91-130, Sept. 2003a.

M. L. Anderson. Representations, symbols, and embodiment. Artificial Intelligence, 149(1):151$156,2003 \mathrm{~b}$.

B. Bell, L. D. Canamero, S. Coradeschi, C. Gomez, A. Saffiotti, C. Tsatsoulis, and T. Walsh. AAAI 2002 fall symposium series reports: Anchoring symbols to sensor data in single- and multiple-robot systems. AI Magazine, Summer, 2002.

J. Bell and Z. Huang. Seeing is believing: A common sense theory of the adoption of perceptionbased beliefs. Artificial Intelligence for Engineering Design, Analysis and Manufacturing, 13: 133-140, 1999.

M. H. Bickhard. Troubles with computationalism. In W. O'Donohue and R. F. Kitchener, editors, The Philosophy of Psychology, pages 173-183. Sage, London, 1996.

D. S. Blank, D. Kumar, and L. Meeden. A developmental approach to anchoring. Technical Report 2002-01, Bryn Mawr College, Computer Science, Bryn Mawr, PA, USA, 2002.

I. Bloch and A. Saffiotti. Some similarities between anchoring and pattern recognition concepts. In Proc. AAAI-01 Fall Symposium Series on 'Anchoring Symbols to Sensor Data in Single and Multiple Robot Systems', Nov 2-4 2001, North Falmouth, MA, USA, 2001.

A. Bonarini, M. Matteucci, and M. Restelli. Anchoring: do we need new solutions to an old problem or do we have old solutions for a new problem? In Proc. AAAI-01 Fall Symposium Series on 'Anchoring Symbols to Sensor Data in Single and Multiple Robot Systems', Nov 2-4 2001, North Falmouth, MA, USA, pages 76-86, 2001a.

A. Bonarini, M. Matteucci, and M. Restelli. Concepts for anchoring in robotics. In F. Esposito, editor, AI*IA 2001: Advances in Artificial Intelligence: 7th Congress of the Italian Association for Artificial Intelligence; Bari, Italy; Sept 25-28, 2001. Springer, Berlin, 2001b.

L. BonJour. Epistemological problems of perception. In E. N. Zalta, editor, The Stanford Encyclopedia of Philosophy. Fall edition, 2001. URL http://plato.stanford.edu/archives/fall2001/entries/perception-episprob/.

A. Bouguerra, L. Karlsson, and A. Saffiotti. Situation assessment for sensor-based recovery planning. In Proc. 17th European Conf. on Artificial Intelligence (ECAI-06), Aug 29-Sept 1 2006, Riva del Garda, Italy, pages 673-677, 2006. 
N. Bredeche, Y. Chevaleyre, J.-D. Zucker, A. Drogoul, and G. Sabah. A meta-learning approach to ground symbols from visual percepts. Robotics and Autonomous Systems, 43(2-3 special issue on 'Perceptual Anchoring: Anchoring Symbols to Sensor Data in Single and Multiple Robot Systems'):149-162, May 2003.

M. Broxvall, S. Coradeschi, L. Karlsson, and A. Saffiotti. Have another look: On failures and recovery planning in perceptual anchoring. In Proc. 16th European Conf. on Artificial Intelligence (ECAI-04), Aug 22-27 2004, Valencia, Spain, 2004a. Workshop on Cognitive Robotics.

M. Broxvall, L. Karlsson, and A. Saffiotti. Steps toward detecting and recovering from perceptual failures. In Proc. 8th Int. Conf. on Intelligent Autonomous Systems (IAS-8), pages 793-800, 2004b.

M. Broxvall, S. Coradeschi, L. Karlsson, and A. Saffiotti. Recovery planning for ambiguous cases in perceptual anchoring. In Proc. 20th Nat. Conf. on Artificial Intelligence (AAAI-05), Pittsburgh, $P A$, USA, pages 1254-1260. AAAI, 2005.

A. Chella, S. Coradeschi, M. Frixione, and A. Saffiotti. Perceptual anchoring via conceptual spaces. In Proc. AAAI-04 Workshop on Anchoring Symbols to Sensor Data, San Jose, CA, USA, Jul $262004,2004$.

J. J. Clark and A. L. Yuille. Data Fusion for Sensory Information Processing Systems. Kluwer Academic, Norwell, MA, USA, 1990.

D. Cole. The chinese room argument. In E. N. Zalta, editor, The Stanford Encyclopedia of Philosophy. Fall edition, 2004 URL http://plato. stanford.edu/archives/fall2004/entries/chinese-room/.

S. Coradeschi and A. Saffiotti. Perceptual anchoring of symbols for action. In Proc. 17th Int. Joint Conf. on Artificial Intelligence (IJCAI-01), Seattle, WA, USA, pages 407-412, 2001.

S. Coradeschi and A. Saffiotti. An introduction to the anchoring problem. Robotics and Autonomous Systems, 43(2-3 special issue on 'Perceptual Anchoring: Anchoring Symbols to Sensor Data in Single and Multiple Robot Systems'):85-96, 2003a.

S. Coradeschi and A. Saffiotti. Perceptual anchoring: A key concept for plan execution in embedded systems. In M. Beetz, J. Hertzberg, M. Ghallab, and M. Pollack, editors, Advances in PlanBased Control of Robotic Agents, number 2466 in Lecture Notes in Artificial Intelligence, pages 89-105. Springer, Berlin, 2002.

S. Coradeschi and A. Saffiotti. Anchoring symbols to sensor data: preliminary report. In Proc. 17th Nat. Conf. on Artificial Intelligence (AAAI-00), Austin, TX, USA, pages 129-135. AAAI, 2000 .

S. Coradeschi and A. Saffiotti. Anchoring symbols to vision data by fuzzy logic. In A. Hunter and S. Parsons, editors, Symbolic and Quantitative Approaches to Reasoning and Uncertainty, number 1638 in Lecture Notes in Computer Science, pages 104-115. Springer, 1999.

S. Coradeschi and A. Saffiotti. Perceptual anchoring with indefinite descriptions. In Proc. 1st Joint SAIS-SSLS Workshop, 2003, Örebro, Sweden, $2003 \mathrm{~b}$.

S. Coradeschi, D. Driankov, L. Karlsson, and A. Saffiotti. Fuzzy anchoring. In Proc. IEEE Int. Conf. on Fuzzy Systems, pages 111-114, Melbourne, Australia, 2001.

N. Correll, G. Sempo, Y. Lopez de Meneses, J. Halloy, J.-L. Deneubourg, and A. Martinoli. SwisTrack: A tracking tool for multi-unit robotic and biological systems. In Proc. IEEE/RSJ Int. Conf. on Intelligent Robots and Systems (IROS-06), Oct 9-15 2006, Beijing, China, pages 2185-2191, 2006. 
T. Crane. The problem of perception. In E. N. Zalta, editor, The Stanford Encyclopedia of Philosophy. Spring edition, $2005 . \quad$ URL http://plato.stanford.edu/archives/spr2005/entries/perception-problem/.

P. Davidsson. Toward a general solution to the symbol grounding problem: Combining machine learning and computer vision. In AAAI Fall Symposium Series "Machine Learning in Computer Vision: What, Why, and How?", pages 191-202. AAAI, 1993.

R. Descartes. Discourse on Method, Optics, Geometry, and Meteorology. Bobbs-Merrill, Indianapolis, IN, USA, 1965. Translated by Paul J. Olscamp, originally published in French in 1637.

M. Fichtner, A. Großmann, and M. Thielscher. Intelligent execution monitoring in dynamic environments. Fundamenta Informaticae, 57:371-392, Oct. 2003.

G. Fitch. Singular propositions. In E. N. Zalta, editor, The Stanford Encyclopedia of Philosophy. Summer edition, $2005 . \quad$ URL http://plato.stanford.edu/archives/sum2005/entries/propositions-singular/.

J. Fritsch, M. Kleinehagenbrock, S. Lang, T. Plötz, G. A. Fink, and G. Sagerer. Multi-modal anchoring for human-robot-interaction. Robotics and Autonomous Systems, 43(2-3 special issue on "Anchoring Symbols to Sensor Data in Single and Multiple Robot Systems"):133-147, 2003.

P. Gärdenfors. Conceptual Spaces. Bradford Books, MIT, Cambridge, MA, USA, 2000.

P. Gärdenfors. Knowledge in Flux: Modeling the Dynamics of Epistemic States. MIT, Cambridge, MA, USA, 1988.

E. Gat. Integrating planning and reacting in a heterogenous asynchronous architecture for controlling real-world mobile robots. In Proc. 10th Nat. Conf. on Artificial Intelligence (AAAI-92), San Jose, CA, USA, pages 809-815. MIT, 1992.

M. L. Ginsberg and D. E. Smith. Reasoning about action I: A possible worlds approach. Artificial Intelligence, 35(2):165-195, 1988.

S. O. Hansson. A Textbook of Belief Dynamics. Kluwer Academic, 1997.

S. Harnad. The symbol-grounding problem. Physica D, 42:335-346, 1990.

S. Harnad. Grounding symbols in the analog world with neural networks. Think: special issue on "Connectionism versus Symbolism", 2(1):12-78, 1993.

S. Harnad. Cognition is categorization. In H. Cohen and C. Lefebvre, editors, Handbook of Categorization in Cognitive Science. Elsevier, 1st edition, 2005.

S. Harnad. Symbol grounding and the origin of language. In M. Scheutz, editor, Computationalism: New Directions, pages 143-158. MIT, 2002.

S. Harnad. Transduction, yes. Degrees of grounding, no. Psycoloquy, 12(037), 2001.

J. Haugeland. Artificial Intelligence: The Very Idea. Bradford / MIT, Cambridge, MA, USA, 1985.

F. Heintz and P. Doherty. Managing dynamic object structures using hypothesis generation and validation. In Proc. AAAI-04 Workshop on Anchoring Symbols to Sensor Data, San Jose, CA, USA, Jul 26 2004, 2004a.

F. Heintz and P. Doherty. DyKnow: A framework for processing dynamic knowledge and object structures in autonomous systems. In Proc. Int. Workshop on Monitoring, Security, and Rescue Techniques in Multiagent Systems (MSRAS), Jun 7-9 2004, Plock, Poland, 2004b. 
V. Honavar. Symbolic artificial intelligence and numeric artificial neural networks: Towards a resolution of the dichotomy. In Computational architectures integrating neural and symbolic processes: a perspective on the state of the art, pages 351-388. Kluwer, 1994.

A. S. Hornby. Oxford Advanced Learner's Dictionary of Current English. Oxford University, Oxford, UK, 6th edition, 2000.

M. Huemer. Sense-data. In E. N. Zalta, editor, The Stanford Encyclopedia of Philosophy. Spring edition, 2005. URL http://plato. stanford.edu/archives/spr2005/entries/sense-data/.

F. Jackson. Perception: A Representative Theory. Cambridge University, Cambridge, MA, USA, 1977.

S. A. Jackson and N. E. Sharkey. Grounding computational engines. Artificial Intelligence Review, 10(1-2):65-82, 1996.

Y. Jin and M. Thielscher. Iterated belief revision, revised. Artificial Intelligence, 171(1):1-18, 2007.

L. Karlsson, A. Bouguerra, M. Broxvall, S. Coradeschi, and A. Saffiotti. To secure an anchor - a recovery planning approach to ambiguity in perceptual anchoring. AI Communications, 21(1): $1-14,2008$.

M. Kleinehagenbrock, S. Lang, J. Fritsch, F. Lömker, G. A. Fink, and G. Sagerer. Person tracking with a mobile robot based on multi-modal anchoring. In Proc. IEEE Int. Workshop on Robot and Human Interactive Communication (ROMAN), Berlin; Sept 25-27 2002, 2002.

S. Lang, M. Kleinehagenbrock, S. Hohenner, J. Fritsch, G. A. Fink, and G. Sagerer. Providing the basis for human-robot-interaction: A multi-modal attention system for a mobile robot. In Proc. Int. Conf. on Multimodal Interfaces (ICMI-03), Vancouver, Canada, pages 28-35. ACM, Nov. 2003.

P. Le Morvan. Arguments against direct realism and how to counter them. The American Philosophical Quarterly, 41(3):221-234, 2004.

D. B. Lenat. CYC: a large-scale investment in knowledge infrastructure. Communications of the $A C M, 38(11): 33-38,1995$.

A. Loutfi. Communicating perceptions: Grounding symbols to artificial olfactory signals. Master's thesis, Örebro University, Örebro, Sweden, 2003.

E. Mach. The Analysis of Sensations, and the Relation of the Physical to the Psychical. The Open Court Publishing Company, Chicago, IL, US, 1914. Translated from 1st German edition by C. M. Williams, revised from 5th German edition by Sydney Waterlow.

J. McCarthy. Situations and Actions and Causal Laws. Stanford Artificial Intelligence Project, Memo 2, Stanford University, CA, USA, 1963.

J. McCarthy. Epistemological problems of artificial intelligence. In Proc. Int. Joint Conf. on Artificial Intelligence (IJCAI-77), pages 1038-1044, Cambridge, MA, USA, 1977.

J. McCarthy. Artificial intelligence, logic and formalizing common sense. Philosophical Logic and Artificial Intelligence, pages 161-190, 1989.

J. McCarthy and P. J. Hayes. Some philosophical problems from the standpoint of artificial intelligence. Machine Intelligence, 4:463-502, 1969.

S. McKennoch and L. G. Bushnell. Dynamics of convergence to a symbol anchoring consensus in distributed agents. Technical Report UWEETR-2005-0006, University of Washington, Seattle, WA, USA, 2005. 
H. Murase and S. K. Nayar. Visual learning and recognition of 3D objects from appearance. International Journal on Computer Vision, 14(1):5-24, Jan. 1995.

A. Newell and H. A. Simon. Computer science as empirical inquiry: Symbols and search. Communications of the ACM, 19(3):113-126, 1976.

Plato. The Republic. Book VII. 360 B.C.

E. Prem. Symbol grounding revisited. Technical Report TR-94-19, Austrian Research Institute for Articial Intelligence, Vienna, Austria, 1994.

E. Prem. Fundamentals of growing concept spaces for autonomous sign users. In Proc. Int. Conf. Simulation of Adaptive Behavior (SAB-02), Workshop "On Growing Up Artifacts that Live Basic Principles and Future Trends"; Aug 10 2002, Edinburgh, UK, 2002.

C. Rasmussen. Combining laser range, color, and texture cues for autonomous road following. In Proc. IEEE Int. Conf. on Robotics and Automation (ICRA-02), Apr 22-28 2000, San Francisco, $C A$, USA, pages 4320-4325, 2002.

M. Reimer. Reference. In E. N. Zalta, editor, The Stanford Encyclopedia of Philosophy. Winter edition, 2006. URL http://plato.stanford.edu/archives/win2006/entries/reference/.

D. Roy. Grounding language in the world: Schema theory meets semiotics. Submitted to Artificial Intelligence, 2004.

D. Roy and E. Reiter. Connecting language to the world. Artificial Intelligence, 167(1-2):1-12, 2005.

B. Russell. The Principles of Mathematics. Cambridge University, Cambridge, MA, USA, 1903.

B. Russell. On denoting. Mind XIV, pages 479-493, Oct. 1905.

S. J. Russell and P. Norvig. Artificial Intelligence. A Modern Approach. Prentice Hall Series in Artificial Intelligence. Prentice-Hall, Upper Saddle River, NJ, USA, second international edition, 2003.

D. Schulz, W. Burgard, D. Fox, and A. B. Cremers. Tracking multiple moving objects with a mobile robot. In Proc. IEEE Conf. on Computer Vision and Pattern Recognition (CVPR-01), Kauwai, HI, USA, volume 1, pages 371-377, 2001.

M. Shanahan. Perception as abduction: Turning sensor data into meaningful representation. Cognitive Science, 29:103-134, 2005.

S. C. Shapiro. Artificial intelligence. In A. Ralston, E. D. Reilly, and D. Hemmendinger, editors, Encyclopedia of Computer Science, pages 89-93. Groves Dictionaries, New York, 4th edition, 2000.

M. Steup. Epistemology. In E. N. Zalta, editor, The Stanford Encyclopedia of Philosophy. Fall edition, $2006 . \quad$ URL http://plato. stanford.edu/archives/fall2006/entries/epistemology/.

R. Sun. Symbol grounding: a new look at an old idea. Philosophical Psychology, 13:149-172, 2000.

M. Taddeo and L. Floridi. Solving the symbol grounding problem: a critical review of fifteen years of research. Journal of Experimental and Theoretical Artificial Intelligence, 2005.

The University of Chicago. The Chicago Manual of Style. The University of Chicago, Chicago, IL, USA, 14th edition, 1982.

M. Thielscher. Reasoning Robots: The Art and Science of Programming Robotic Agents, volume 33 of Applied Logic Series. Springer, Dordrecht, The Netherlands, 2005. 
S. Thrun, W. Burgard, and D. Fox. Probabilistic Robotics. MIT, Cambridge, MA, USA, 2005.

A. M. Turing. Computing machinery and intelligence. Mind, 59(236):433-60, Oct. 1950.

P. Vogt. Anchoring of semiotic symbols. Robotics and Autonomous Systems, 43(2-3 special issue on 'Perceptual Anchoring: Anchoring Symbols to Sensor Data in Single and Multiple Robot Systems'):109-120, 2003.

M. Witkowski, D. Randell, and M. P. Shanahan. Deriving fluents from sensor data for mobile robots. In Proc. AAAI-01 Fall Symposium Series on 'Anchoring Symbols to Sensor Data in Single and Multiple Robot Systems', Nov 2-4 2001, North Falmouth, MA, USA, pages 44-51, 2001.

L. Wittgenstein. Tractatus Logico-Philosophicus. Routledge \& Keegan Paul, London, 1961. Translation by D. F. Pears and B. F. McGuinness, originally published in 1921.

M. Wooldridge and N. R. Jennings. Intelligent agents: Theory and practice. The Knowledge Engineering Review, 10(2):115-152, 1995.

T. Ziemke. Rethinking grounding. In A. Riegler, M. F. Peschl, and A. von Stein, editors, Understanding Representation in the Cognitive Sciences, pages 177-190. Plenum Academic / Kluwer, New York, 1999. 


\section{Index}

Action AnchorM, 86

Action AnchorU, 47

AI-complete problem, 13

Anchor, 14, 43

Awareness, 7

Axioms $\Sigma_{F C}, 22$

Axioms $\Sigma_{m m s a}, 95$

Axioms $\Sigma_{u m s a}, 59$

Belief state, 65

Cognitivism, 7

Consistency, 45

Constant $S_{0}, 21$

Constant $\perp, 44$

Constant $\emptyset, 20$

Data association problem, 39

Definite description, 6, 33

Descriptive theory, 6

Direct realism, 7

Embodiment, 10

Fluent, 20

Fluent $A, 43,81$

Fluent Prop, 32

Fluent Offset, 57

Fluent Calculus, 20

Fluent $C, 88$

Foundational axioms of Fluent Calculus, 22

Foundational Axioms $\Sigma_{\text {state }}, 20$

Function $D o, 21$

Function $N o P, 43,81$

Function State, 21

Function $T, 102$

Function $\circ, 20$

Functionality Find, multi-modal, 92

Functionality Find, uni-modal, 54

Functionality New, multi-modal, 93

Functionality New, uni-modal, 56

Functionality Track, multi-modal, 89

Functionality Track, uni-modal, 50

Givenness, 7

Groundedness, 10, 18, 64, 100
Harnad's test for grounded symbol meaning, 11

Hypothesis, 33

Hypothesis space, 37

Immediacy, 7

Indefinite description, 6, 33

Indexical, 6

Indirect realism, 7

Knowledge expression, 22

Knowledge state, 22

Knowledge update axiom, 22

Macro Holds, 20

Macro Knows, 22

Macro GroundingInt, 52

Macro NewSymbol, 57

Macro PAssoc, 83

Macro $\Delta_{F}, 54,93$

Macro $\Delta_{N}, 57,94$

Macro $\Delta_{T}, 51,89$

Multi-modal perception, 77

Multi-modal symbol anchoring axioms, 95

Naive realism, 7

Object, 5

Object constancy problem, 40

Object identification, 39

Object permanence phenomenon, 53

Object recognition, 38

Object tracking, 38

Objective perception, 15

Percept, 14, 41, 80

Perception, 8

Perceptual attributes, 14

Perceptual seeming, 7

Perceptual signature, 18, 39

Phenomenalism, 7

Physical object, 5

Precondition axiom, 21

Predicate BState, 66

Predicate BStateSet, 68

Predicate KState, 22

Predicate AnchorUpdate, 91 
Predicate AttrTypeStatic, 30

Predicate $C C, 49$

Predicate Consistent, 45, 82

Predicate DiffCorr, 66

Predicate Grounded, 30

Predicate Intersect, 52

Predicate Is Grounded, 65, 100

Predicate IsNewSymbol, 71

Predicate Match, 51, 90

Predicate Poss, 21

Predicate PBlock, 83

Predicate $P, 41,80$

Predicate PFusion, 95

Predicate PKnown, 90

Predicate Pref, 66

Predicate PrefPercKS, 71

Predicate PrefSets, 67

Predicate UpdatePS, 55

Proper name, 6

Representation problem, 64

Representationalism, 7

Sense-data theory, 7

Sensor fusion, 78

Set $\widehat{\Pi}_{s}, 83$

Singular proposition, 6

Situatedness, 10

Situation, 21

Situation formula, 21

Sort ACTION, 21

Sort FLUENT, 20

Sort ATTR, 30

Sort SIT, 21

Sort OBJCAT, 26

Sort PERCID, 26

Sort PERCPRED, 30

Sort PERCSig, 42, 82

Sort STATE, 20

Sort $\mathcal{X}, 26,81$

State, 20

State formula, 21

State update axiom, 21

Subjective perception, 15

Symbol Anchoring Problem, 13, 18

Symbol grounding, 9

Symbol Grounding Problem, 8

Symbolic description, 31

Taddeo and Floridi's symbol grounding requ., 11

Total Turing Test, 10

Uni-modal perception, 41
Uni-modal symbol anchoring axioms, 59

Z-condition, 11

Zero Semantical Commitment Condition, 11 